\title{
Kinetic Dissection of Translation Initiation in Prokaryotes.
}

\author{
Dissertation \\ for the award of the degree \\ “Doctor rerum naturalium" (Dr. rer. nat.) \\ Division of Mathematics and Natural Sciences of \\ the Georg-August-Universität Göttingen
}

Submitted by

Liudmila Filonava

from Minsk, Belarus

Göttingen, 2013 
Members of the Thesis Committee:

Prof. Dr. Marina V. Rodnina (1st reviewer)

Department of Physical Biochemistry

Max Planck Institute for Biophysical Chemistry

Prof. Dr. Ralf Ficner (2nd reviewer)

Department of Molecular Structural Biology

Göttingen Center for Molecular Biosciences

Prof. Dr. Kai Tittmann

Department of Bioanalytics

Göttingen Center for Molecular Biosciences

Date of oral examination: June 18th, 2013. 


\section{Affidavit}

I hereby declare that I prepared the dissertation "Kinetic Dissection of Translation Initiation in Prokaryotes" on my own and with no other sources and aids than quoted.

Liudmila Filonava

Göttingen, April 30 ${ }^{\text {th }}, 2013$ 


\section{Contents}

Summary

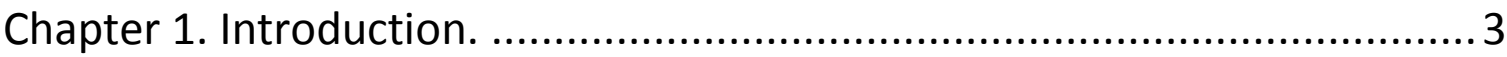

1.1. Protein synthesis in the living cell. The ribosome............................... 3

1.2. The four main phases of translation................................................. 4

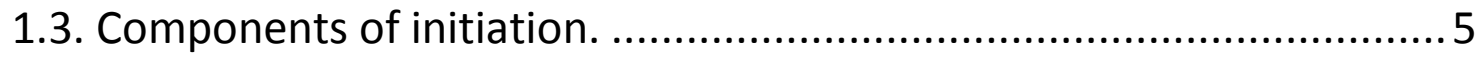

IF1

IF2

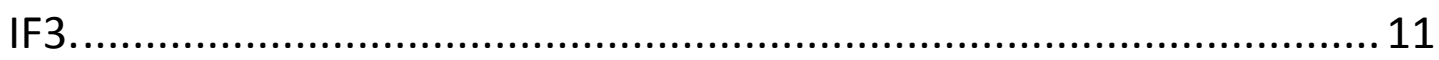

Initiator tRNA

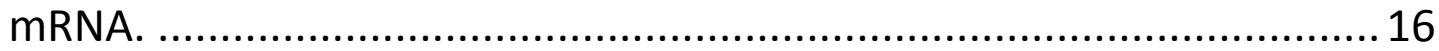

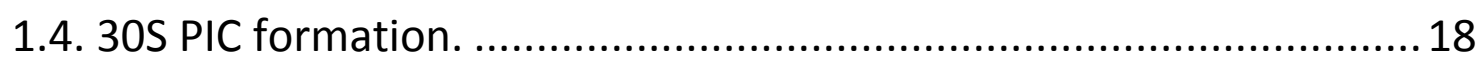

1.5 Transition to the 30S IC. Start codon recognition...............................24

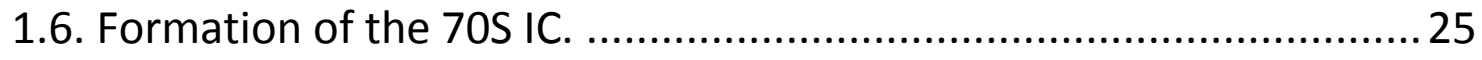

Chapter 2. Kinetics of mRNA binding to the 30S IC and 30S PIC...............27

Chapter 3. Kinetics of GTP hydrolysis by IF2 ...........................................5

Chapter 4. Reconstitution, Functional Analysis and Structural Studies of the Translation Initiation Complex of $T$. thermophilus. ................................... 78

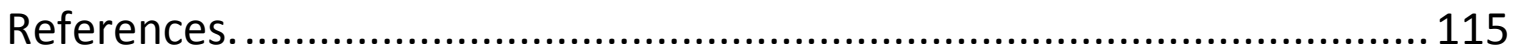

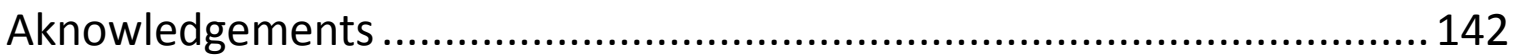

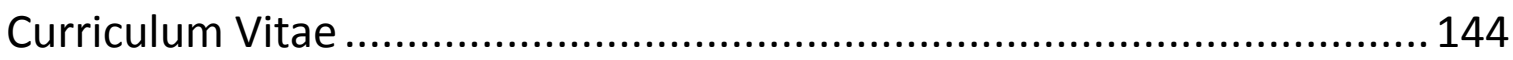




\section{Summary.}

Ribosomes are nucleoprotein complexes which synthesize proteins in the cells. The process of translation can be subdivided into several phases: translation initiation, elongation, termination and ribosome recycling. The prokaryotic initiation system requires the small and large ribosomal subunits ( $30 \mathrm{~S}$ and $50 \mathrm{~S}$ subunits, respectively), initiator tRNA (fMet-tRNA ${ }^{\text {fMet }}$ ), mRNA, and three initiation factors IF1, IF2, IF3. There are two main steps in initiation. During the first step, fMet-tRNA ${ }^{\text {fMet }}$ and mRNA bind the $30 \mathrm{~S}$ subunit with the help of initiation factors forming a $30 \mathrm{~S}$ initiation complex (IC). In the second step, the 50S subunit binds the 30 S IC, whereas IF1 and IF3 dissociate from the complex in a stepwise manner. The docking of the 50S subunit triggers GTP hydrolysis by IF2 which leads to the dissociation of the latter. The resulting complex is able to progress to elongation.

During the first part of the present work GTPase activity of IF2 was studied. We measured the rate of GTP hydrolysis by IF2 using a novel fluorescence-based assay. These measurements, in conjunction with the experiments on dissociation of IF1 and IF3, allowed for placing the GTPase reaction in the model of late events in translation initiation. The rate of GTP did not depend on either substrate, or the 50 s subunit concentration. Based on these observations we suggest that IF2 undergoes conformational rearrangements after the $50 \mathrm{~S}$ subunit docking possibly involving the GTPase activation.

In the second part of the present work influence of mRNA regulatory elements (ShineDalgarno (SD) sequence, initiation codon) on the mRNA binding to the 305 subunit was studied. Our work shows that the primary arrival of mRNA to the ribosome is independent from its TIR structure. TIR was shown to play an important role on the later steps of mRNA stabilization on the 30S IC. We also found that the presence of initiation factors and/or fMet-tRNA ${ }^{\text {fMet }}$ in the $30 \mathrm{~S} \mathrm{IC} \mathrm{does} \mathrm{not} \mathrm{influence} \mathrm{the} \mathrm{primary} \mathrm{docking} \mathrm{of} \mathrm{the}$ mRNA. We conclude that during the assembly of 30S PIC mRNA binds to the complex in parallel but independent on the other components. We also showed that there is an affinity switch upon start codon recognition. mRNAs with AUG codon in the P site are stabilized in the 30S PIC if fMet-tRNA ${ }^{\text {fMet }}$ is present. Thus, on the basis of kinetic constants determined in the current work, we identified potential checkpoint for mRNA selection during translation initiation. 
The aim of the third part of the present project was to crystallize translation initiation complex which consists of 705 ribosome, IF2-GDPNP, mRNA, fMet-tRNA ${ }^{\text {fMet }}$. To date there is no structure of the prokaryotic 70S IC solved by X-ray analysis. It is known from cryo-EM studies that IF2 undergoes structural rearrangements on the ribosome after 50 subunit joining to 30S IC and after the event of GTP hydrolysis. Solving the structure of 7OS IC by X-ray analysis would provide important information about the conformation and contacts of IF2 with the ribosome during the dynamic process of translation initiation. In the present work we reconstituted the translation initiation system from thermophilic bacterium Thermus thermophilus and developed the procedure for 70S IC purification for crystallization purposes. 


\section{Chapter 1. Introduction.}

\subsection{Protein synthesis in the living cell. The ribosome.}

Proteins are essential components of all living cells as they are involved in every cellular process. Proteins are synthesized in the cells according to the information encoded in the messenger RNA (mRNA). Each triplet of mRNA bases (codon) codes for one amino acid. Protein synthesis is catalyzed by the ribosome, which is the large ribonucleoprotein particle, with the help of translational factors and transfer RNAs (tRNAs). The latter serves as an adaptor molecule carrying an amino acid to the active site on the ribosome where the formation of the peptide bond is catalyzed. Recognition of the mRNA codon is accomplished by interaction with the tRNA anticodon.

The prokaryotic $70 \mathrm{~S}$ ribosome has a molecular weight of about $2.5 \mathrm{MDa}$. It consists of the small (30S) and large (50S) ribosomal subunits (Fig. 1.1). The small ribosomal subunit consists of 16S rRNA and 21 proteins, designated form S1 to S21. The decoding center where the recognition of the mRNA codons by the anticodon of the tRNA takes place is located on the $30 \mathrm{~S}$ subunit. The structure of the $30 \mathrm{~S}$ subunit can be subdivided into the four main domains: the body, the shoulder, the platform and the head. (Wimberly et al., 2000). The domains are flexible and can move relative to each other. The decoding center is located in the intersubunit cleft between the head and the platform of the ribosome (Yusupov et al., 2001; Yusupova et al., 2001). The large ribosomal subunit is built up $23 \mathrm{~S}$ rRNA and $5 \mathrm{~S}$ rRNA, as well as 31 proteins. The main function of the $50 \mathrm{~S}$ subunit is catalysis of the peptide bond formation which happens in the peptydiltransferase center. In c ontrast to the $30 \mathrm{~S}$ subunit, the large ribosomal subunit forms a monolithic structure where different RNA domains interact mostly with each other.

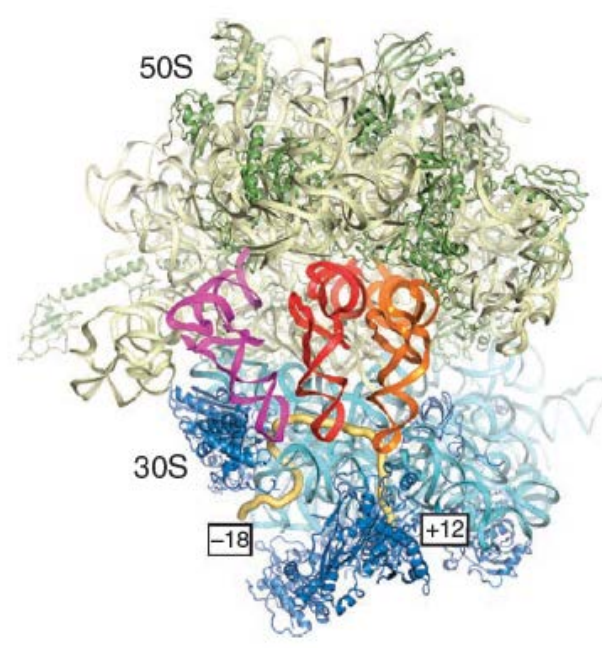

Figure 1.1 (from Jenner et al., 2010) The 70S ribosome with three tRNAs and mRNA. $A, P$, and $E$ site tRNAs are shown in orange, red, and magenta, respectively. Messenger RNA is shown in yellow. $23 \mathrm{~S}$ and $5 \mathrm{~S}$ rRNA are pale green, and 16S rRNA is pale cyan. $50 \mathrm{~S}$ and $30 \mathrm{~S}$ ribosomal proteins are indicated in green and blue, respectively. Parts of the head of the $30 \mathrm{~S}$ subunit have been removed for clarity. 
Three binding sites for tRNA have been identified on the ribosome: the aminoacyl-tRNA binding site ( $A$ site), the peptidyl-tRNA binding site ( $P$ site) and the exit site for the deacylated tRNA (E site) (Fig. 1.1) (Demeshkina et al., 2012; Selmer et al., 2006; Yusupov et al., 2001). The ribosome is considered to be a ribozyme as the peptidyltransferase center and the decoding center are built entirely from RNA, and proteins are not involved in the catalysis of peptide bond formation (Nissen, 2000). Generally, the inner core of the ribosome and the intersubunit space consists mainly of the rRNA, whereas the proteins are located mostly at the outer surface of the ribosome (reviewed in (Bashan and Yonath, 2008; Schmeing and Ramakrishnan, 2009; Steitz, 2008)).

\subsection{The four main phases of translation.}

Protein synthesis occurs in four main phases: initiation, elongation, termination and recycling (Fig. 1.2). During initiation, fMet-tRNA $^{\text {fMet }}$ is positioned at the start codon of the mRNA in the P site of the small ribosomal subunit with the help of three initiation factors (IF1, IF2 and IF3). Translation initiation starts with assembly of three initiation factors, fMet-tRNA ${ }^{\text {fMet }}$ and mRNA on the 30S subunit (Gualerzi and Pon, 1990), thereby forming a $30 \mathrm{~S}$ pre-initiation complex (PIC). Upon binding of each component, conformation rearrangements which affect the entire complex occur. After the recognition of the start codon on the mRNA by fMet-tRNA ${ }^{\text {fMet }}$, 30S PIC is converted to 30S IC. The latter is ready for $50 \mathrm{~S}$ subunit joining thereby forming the 70S IC (Antoun et al., 2006a; Grigoriadou et al., 2007a; Marshall et al., 2009; Milon et al., 2008; Tomsic et al., 2000). Subsequently, the initiation factors dissociate from the ribosome, the start codon recognition takes place and the complex is ready to progress into elongation. The $70 S$ IC then enters the elongation cycle, where the protein is synthesized by adding one amino acid at a time to a growing peptide chain. Each time the elongation factor Tu (EFTu) in complex with GTP delivers a new aminoacyl-tRNA (aa-tRNA) into the A site of the ribosome. After the accommodation of the correct aa-tRNA, peptide bond formation takes place. The peptidyl chain from the P site tRNA is transferred to the A site tRNA, resulting in the extension of the peptide by one amino acid. The formation of peptide bond is followed by translocation, when the ribosome moves by one codon triplet relative to the mRNA and tRNAs. Translocation is catalyzed by EF-G in complex with GTP. Upon binding to the ribosome, EF-G hydrolyses GTP which drives the conformational rearrangements on the ribosome leading to the translocation movement (Peske et al., 
2004; Rodnina et al., 1997; Savelsbergh et al., 2003). During translocation, the peptidyltRNA is displaced from the $A$ site to the $P$ site, and deacylated tRNA moves form the $P$ site to the $E$ site and then dissociates. The next mRNA codon appears in the vacant $A$ site after dissociation of EF-G from the ribosome. When the complete peptide has been synthesized, the stop codon is presented in the A site and the translation is terminated with the help of release factors (RF). The stop codon UAG is recognized by RF1, UGA by RF2, and UAA by both, RF1 and RF2. The release factors trigger hydrolysis of the peptide chain from the peptidyl-tRNA in the P site. Dissociation of RF1 and RF2 from the ribosome is catalyzed by RF3 in the GTP-dependent manner. The resulting posttermination complex retains mRNA and deacylated tRNA in the $P$ site of the ribosome. Prior the new translation initiation round, the posttermination complex is disassembled with the help of EF-G, ribosomal recycling factor (RRF) and IF3. RRF and EF-G promote subunit dissociation and IF3 destabilizes deacylated tRNA in the P site, stimulates its dissociation from the 30S and prevents subunit association (Peske et al., 2005). After the recycling step, the ribosomal subunits can bind new mRNA and start synthesis of a new protein.

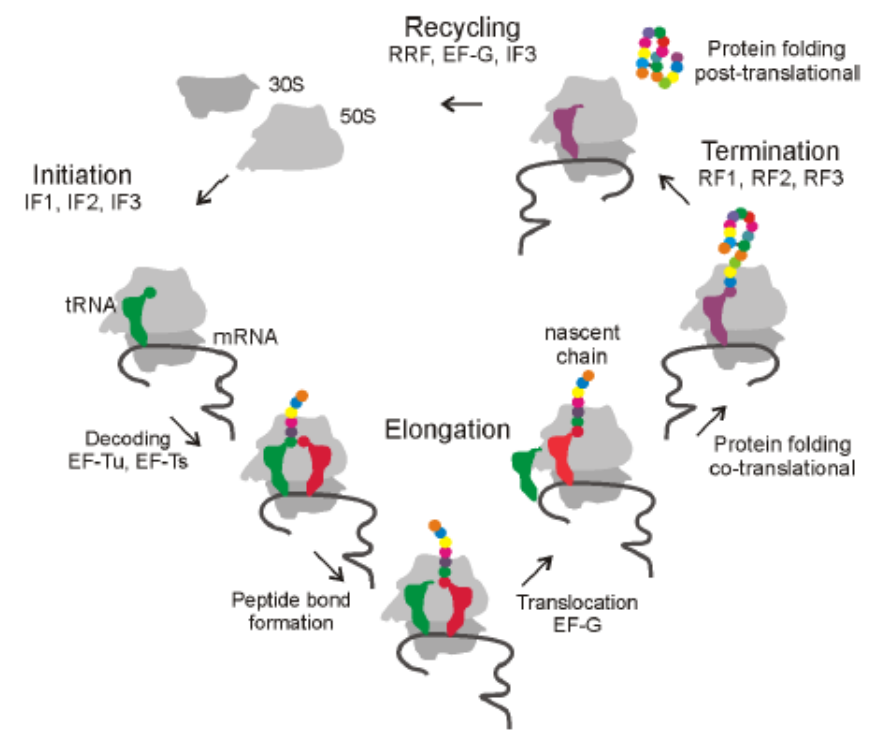

Figure 1.2 The four main phases of translation: initiation, elongation, termination and recycling. See explanations in the text.

\subsection{Components of initiation.}

IF1.

Translation initiation factor 1 (IF1) is a small globular protein of 71 amino acids (8.1 kDa). F1 is coded by infA gene which is found in all prokaryotic genomes sequenced including 
chloroplasts (Hirose et al., 1999). Interestingly, infA is not present in the genomes of mammalian mitochondria where its function is performed by IF2 (Gaur et al., 2008). Bacterial IF1 has homologs in all kingdoms of life - alF1A in archaea and elF1A in eukaryotes (Kyrpides and Woese, 1998; Sørensen et al., 2001). IF1 was shown to be essential for cell viability (Antoun et al., 2006b; Croitoru et al., 2004; Cummings and Hershey, 1994). There is evidence that IF1 it is not crucial in the in vitro translation system based on purified components, in contrast to the other translation initiation factors IF2 and IF3 (Shimizu et al., 2001).

Structure of IF1 from Escherichia. coli was determined by NMR (Fig. 1.3) (Sette et al., 1997). Later 30S ribosomal subunit in complex with IF1 was crystallized (Carter et al., 2001). IF1 was found to have structural similarities with ribosomal protein S1, bacterial cold shock proteins CspA and CspB, polynucleotide phosphorylase, $\mathrm{N}$-terminal domain of aspartyl-tRNA synthetase and the staphylococcal nuclease (Gribskov, 1992; Sette et al., 1997).These proteins share a common motif called oligomer binding fold (OB-fold). The OB-fold has a five-stranded $\beta$-sheet coiled to form a $\beta$-barrel. The $3 r d$ and the 4 th strands are connected by an $\alpha$-helix which makes the whole structure more flexible than the typical $\beta$-barrel (Murzin, 1993).

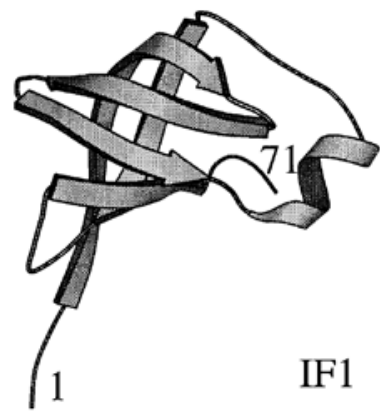

Figure 1.3 (From Sette et al., 1997) The structure of IF1 from E. coli determined with multidimensional NMR spectroscopy. The structure of IF1 is characterized by a five-stranded $\beta$-barrel. The fold of IF1 is very similar to that found in the bacterial cold shock proteins $\mathrm{CspA}$ and $\mathrm{CspB}$, the $\mathrm{N}$-terminal domain of aspartyl-tRNA synthetase and the staphylococcal nuclease, and can be identified as the oligomer-binding motif.

Some of the proteins from the OB-fold protein family showed nucleic acid binding properties. As IF1 was shown to bind synthetic polynucleotides in solution (Schleich et al., 1980), it was suggested that IF1 plays its role in initiation by interacting with RNA. In fact, IF1 binds to the 30S subunit by electrostatic interactions (Celano et al., 1988). IF1 location on the ribosome and residues involved in 30S-IF1 interaction were identified by site-directed mutagenesis and crosslinking, NMR spectroscopy and crystallography (Boileau et al., 1983; Carter et al., 2001; Croitoru et al., 2005; Dahlquist and Puglisi, 2000; Gualerzi et al., 1989; Moazed et al., 1995; Sette et al., 1997). In was consistently found that IF1 binds 30S ribosomal subunit in the A site region (Dahlquist and Puglisi, 2000; Moazed et al., 1995). A crystal structure analysis showed more precise location of 
IF1 on the 30 S ribosomal subunit in a cleft between helix 44, the 530 loop of 16S RNA, and the ribosomal protein S12 (Carter et al., 2001). The binding of IF1 to the 30S subunit induces conformational changes in the region near the $A$ site which lead to the more global conformational change of the $30 \mathrm{~S}$ subunit resulting in a tilt of the head of the $30 \mathrm{~S}$ towards the A site.

Functions of IF1 in translation initiation. One of the proposed functions of IF1 in translation initiation is stimulation of the action of the other initiation factors IF2 and IF3 (Antoun et al., 2006a; Laursen et al., 2005; Pon and Gualerzi, 1984; Wintermeyer and Gualerzi, 1983). IF1 stimulates IF2 binding to the ribosome (Caserta et al., 2006; Celano et al., 1988; Gualerzi and Pon, 1990; Milón et al., 2012a; Moreno et al., 1999; Stringer et al., 1977; Weiel and Hershey, 1982). IF1 interacts with IF2 on the ribosome (Allen et al., 2005; Boileau et al., 1983; Brock et al., 1998) which is probably how stabilization of IF2 on the ribosome is achieved; the reverse effect is also true - IF1 was shown to be stabilized on the ribosome in the presence of IF2 (Caserta et al., 2006; Celano et al., 1988; Milón et al., 2012a; Stringer et al., 1977; Weiel and Hershey, 1982). The interaction between IF1 and IF2 was suggested to be universal as similar interaction is observed also in the eukaryotic system (Chaudhuri et al., 1997; Roll-Mecak et al., 2000). Recycling of IF2 on/off $70 S$ is also effected by IF1 as it alters the thermodynamic stability of the IF2-30S complex (Chae et al., 1969; Kay and Grunberg-Manago, 1972; Stringer et al., 1977; Voorma et al., 1979).

IF1 also stimulates the binding of MMet-tRNA $^{\text {fMet }}$ to the P site (Antoun et al., 2006b; Kay and Grunberg-Manago, 1972; Mukundan et al., 1968; Voorma et al., 1979). Together with IF2 it has a role in recognition of formylmethionine moiety of fMet-tRNA ${ }^{\text {fMet }}$ by IF2 and discrimination against deacylated or deformylated tRNAs (Antoun et al., 2006a). Moreover, IF1 has some role in increasing the binding of mRNA to the initiation complex in the presence of IF2 (Studer and Joseph, 2006).

Prokaryotic IF1 was purified and characterized from three bacterial species, E. coli, $T$. thermophilus and Bacillus stearothermophilus; the latter two are thermophilic species (Hershey et al., 1977; Kapralou et al., 2009; Wolfrum et al., 2003). Curiously, thermophilic IF1 does not promote binding of IF2 to the 30S ribosomal subunit unlike IF1 from E. coli (Kapralou et al., 2008), probably because thermophilic IF2 has very short Nterminal domain, with which IF1 may interact (Laursen et al., 2005).

Other functions of IF1. It was observed that cellular level of IF1 is increased two- to threefold under the cold shock conditions (Giuliodori et al., 2004). Some mutations in 
the infA gene coding for IF1 lead to cold sensitivity in bacteria (Croitoru et al., 2004). The structure of IF1 is similar to that of cold shock proteins (CSP) in E. coli and it was shown that IF1 can compensate for CSPs in vivo (Sette et al., 1997). It is also known that IF1 can bind synthetic polynucleotides in solution (Schleich et al., 1980). Based on these observations, IF1 is suggested to have a function of an RNA chaperone (Phadtare et al., 2007; Sette et al., 1997) and transcriptional antiterminator (Phadtare et al., 2007).

IF2.

IF2 is the largest of three bacterial initiation factors. It is a GTPase that consists of several domains (Iwasaki et al., 1968; Revel et al., 1968). IF2 is 890 amino acids long and has molecular weight of $97.35 \mathrm{kDa}$. IF2 is coded by infB and was shown to be essential (Laalami et al., 1991; Plumbridge et al., 1982). Homologs of IF2 were found in archaea (alF5B) and in eukaryotes (elF5B) (Kyrpides and Woese, 1998; Sørensen et al., 2001). IF2, alF5B and elF5B were shown to have similar functions in initiation (Choi et al., 1998; Pestova et al., 2000). The GTP binding domain of IF2 shares significant homology with that of other translational GTPases EF1A, EF2, RF3, and SelB (Rodnina et al., 2000; Vetter and Wittinghofer, 2001).

IF2 exists in the cell in three isoforms: IF $2 \alpha(97.3 \mathrm{kDa})$, IF $2 \beta(79.7 \mathrm{kDa})$ and IF2 $\gamma(78.8$ kDa) (Miller and Wahba, 1973; Morel-Deville et al., 1990; Nyengaard and Mortensen, 1991; Plumbridge and Deville, 1985; Sacerdot et al., 1992). The isoforms are generated by translation from 3 alternative start codons within infB (Mortensen et al., 1995). IF2 $\alpha$ is the full-length protein, IF2 $\beta$ lacks first 157 amino acids, and IF $2 \gamma$ lacks first 165 amino acids. All the isoforms are required for optimal growth and all 3 are present in approximately equal amounts in the cell at normal growth conditions (Howe and Hershey, 1983; Sacerdot et al., 1992). However the ratio of IF2 $\beta$ and IF2 $\gamma$ to IF2 $\alpha$ was shown to increase in cold-shock conditions (Giuliodori et al., 2004).

At present, the structure of the full-length bacterial IF2 is not available. The crystal structure of its archaeal homolog elF5B from Methanobacterium thermoautotrophicum has been solved (Fig. 1.4) (Roll-Mecak et al., 2000). However, there is data that suggests that the behavior of bacterial IF2 is different from its archaeal homolog alF5B (Wienk et al., 2012). The current knowledge about the prokaryotic IF2 structure is based on the results of partial proteolysis experiments (Gualerzi et al., 1991), homology models and sequence alignments (Allen et al., 2005; Julián et al., 2011; Simonetti et al., 2008). Cryo- 
EM reconstruction of full-length IF2 from $E$. coli in complex with 30 S ribosomal subunit is also available (Julián et al., 2011).

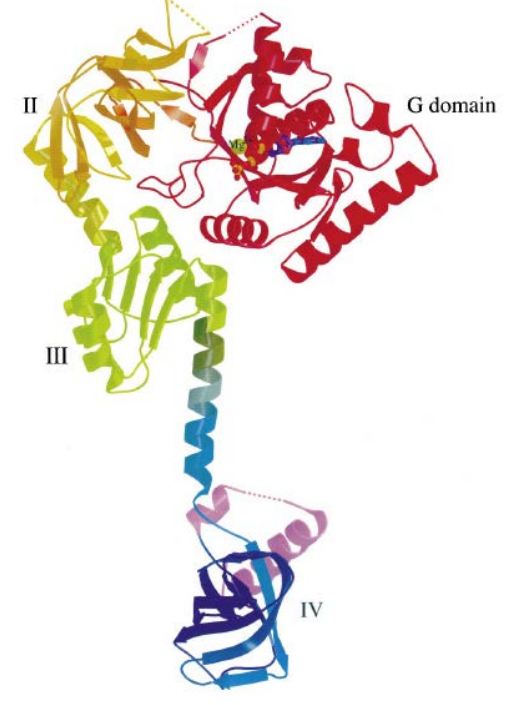

Figure 1.4 (From Roll-Mecak et al., 2000) Structure of M. thermoautotrophicum IF2/elF5B.GTP. Ribbons diagram showing the ventral or nucleotide binding site view. Domain color coding is as follows: $G$ domain, red; domain II, yellow; domain III, green; domain IV EF-Tu-type $\beta$-barrel, blue; C-terminal $\alpha$ - helices, magenta. The bound nucleotide is shown as a ball and stick atomic model, and the $\mathrm{Mg}^{2+}$ ion is denoted by a labeled green sphere.

Prokaryotic IF2 consists of three large domains, each divided further into sub-domains (Gualerzi et al., 1991; Misselwitz et al., 1997; Mortensen et al., 1998; Zoldák et al., 2008): the N-terminal domain (NTD; sub-domains N1 and N2), the GTP-binding domain (G-domain; sub-divided into G1, G2 and G3) and the C-terminal domain (CTD; subdomains $\mathrm{C} 1$ and $\mathrm{C} 2$ ). The nucleotide binding site of the G-domain contains the switch I and switch II regions which allow for discrimination of GTP and GDP. The G-domain and the CTD are highly conserved among the species; whereas the NTD can vary in its length and amino acid sequence (Steffensen et al., 1997).

The structures of IF2 subdomains C1, C2, G2 and the NTD fragment were solved by NMR spectroscopy (Laursen et al., 2003; Meunier et al., 2000; Wienk et al., 2005, 2012). The structure of the CTD fragment of IF2 was described using small-angle X-ray scattering (SAXS) (Rasmussen and Oliveira, 2008). The CTD of IF2 consists of two modules - C1 and C2 (Misselwitz et al., 1997). No particular function is assigned to C1 yet. C2 recognizes and binds fMet-tRNA ${ }^{\mathrm{fMet}}$ (Guenneugues et al., 2000; Spurio et al., 2000; Szkaradkiewicz et al., 2000).

The NTD of IF2 enhances the interaction of the factor with the $30 \mathrm{~S}$ and $50 \mathrm{~S}$ subunits; it increases the factor's affinity to the ribosome but is not absolutely necessary for the interaction as was shown in vitro and in vivo under optimal growth conditions (Caserta et al., 2006, 2010; Laalami et al., 1991; Moreno et al., 1999). The isolated NTD is capable of binding to the $30 \mathrm{~S}$ subunit (Moreno et al., 1999; Mortensen et al., 1998). In the 
complex of full-length IF2 with the 30S subunit, the NTD interacts with IF1 and S12 (Boileau et al., 1983; Julián et al., 2011). NTD is connected to the other IF2 domains via flexible linker (Laursen et al., 2004).

The G-domain of IF2 is quite large (about $42 \mathrm{kDa}$ ) and consists of three subdomains. The function of G1 is not yet clear. In some of the bacterial IF2s and in archaeal homolog alF5B G1 is absent (Roll-Mecak et al., 2000). G2 binds GTP and interacts with the 50S subunit; G3 interacts with $30 \mathrm{~S}$ and is present in all translational GTPases (Allen et al., 2005; Caserta et al., 2010; Julián et al., 2011; Marzi et al., 2003; Simonetti et al., 2008).

IF2 position on the ribosome was mapped using cross-linking (Bollen et al., 1975), footprinting (La Teana et al., 2001), cleavage of the rRNA by chemical nucleases tethered to IF2 (Marzi et al., 2003) and cryo-EM reconstructions (Allen et al., 2005; Julián et al., 2011; Myasnikov et al., 2005; Simonetti et al., 2008). IF2 was shown to have two binding sites on the 30S subunit (Caserta et al., 2006) making contacts to 16S rRNA and S12 (Julián et al., 2011). On the 50S subunit, IF2 interacts with the L7/L12 stalk, the sarcinricin loop of 23 SRNA and L11 (Fakunding et al., 1973; Helgstrand et al., 2007). The contact to the L7/L12 stalk was shown to be important for IF2-mediated subunit association (Mandava et al., 2012). IF2 binding site on the ribosome overlaps with the binding site of other translational GTPases. Rearrangement of the ribosome upon binding of IF2 was observed (Allen et al., 2005; Julián et al., 2011; Myasnikov et al., 2005; Simonetti et al., 2008). IF2 was shown to change its localization after binding of the 50S to the 30S IC (Allen et al., 2005; Julián et al., 2011; Marzi et al., 2003; Myasnikov et al., 2005; Simonetti et al., 2008). However, crystal structures of IF2 in complex with the 30S subunit and the $70 \mathrm{~S}$ ribosome are not available at the moment. Detailed atomic resolutions structures would help better understanding of the global role of IF2 in the translation initiation process.

The GTPase activity. IF2-dependent GTP hydrolysis is triggered during translation initiation by 50 S subunit joining the 30 S IC. (Luchin et al., 1999; Tomsic et al., 2000). IF2 has no intrinsic GTPase activity. But IF2 from B. stearothermophilus was shown to hydrolyze GTP in $20 \%$ ethanol in the absence of ribosomes (Severini et al., 1991). It was shown by isothermal titration calorimetry and SAXS that IF2 changes conformation depending on the nucleotide bound (GTP or GDP) or nucleotide-free form (Hauryliuk et al., 2009; Pon et al., 1985; Vohlander Rasmussen et al., 2011). IF2 has $1 \mu \mathrm{M}$ affinity for GDP and affinity ranging between $10 \mu \mathrm{M}$ and $40 \mu \mathrm{M}$ fot GTP (Hauryliuk et al., 2009; Milon et al., 2006; Pon et al., 1985). Due to the only 10 times difference in GDP/GTP 
affinities to IF2 and the high rate of nucleotide dissociation, the nucleotide exchange is spontaneous and guanine exchange factor is not required (Milon et al., 2006). The role of GTP hydrolysis in translation initiation is not yet entirely clear although several ideas are suggested, which are reviewed in more detail in Chapter 2 of the present work.

Functions in initiation. IF2 is responsible for recognition of the formyl group of fMettRNA $^{\text {fMet }}$ (Antoun et al., 2006b; Majumdar et al., 1976; Sundari et al., 1976). IF2 in the GTP form promotes the recruitment of fMet-tRNA ${ }^{\text {fMet }}$ to the $30 \mathrm{~S}$ PIC (Milon et al., 2010) and ensures adjustment of initiator tRNA in the ribosomal P-site (Antoun et al., 2006b; Lockwood et al., 1971; La Teana et al., 1996) and mRNA restructuring during the formation of 30S IC (Studer and Joseph, 2006). IF2 in complex with GTP or its nonhydrolysable analog GDPNP was shown to promote fast association of the ribosomal subunits, whereas the subunits association in presence of IF2-GDP is slow (Antoun et al., 2003).

Other functions of IF2. IF2 is also involved in translation initiation on leaderless mRNAs (Grill et al., 2000, 2001) and translation re-initiation on a downstream ORF of polycistronic mRNAs (Yoo and RajBhandary, 2008). IF2 was suggested to function like a metabolic sensor (Milon et al., 2006). Under stress conditions IF2 binds the alarmone ppGpp. The complex of IF2-ppGpp binds to the initiation complexes and blocks further translation (Milon et al., 2006). The expression of IF2 is increased during the cold-shock (Bae et al., 2000) and osmotic stress conditions (Weber et al., 2006). Moreover IF2 has functions of molecular chaperone, increasing the refolding of unfolded proteins and protecting proteins from thermal denaturation (Caldas et al., 2000). It was proposed that IF2 assists in protein folding and renaturation in the cytoplasm (Caldas et al., 2000).

IF3.

Initiation factor 3 from $E$. coli is a $20.4 \mathrm{kDa}$ protein which consists of 180 amino acids (Olsson et al., 1996; Sabol et al., 1970; Sacerdot et al., 1982). It is the least conserved of all three prokaryotic initiation factors. For example, IF3 from chloroplasts of Euglena gratilis contains the only one central region domain that is homologous to the IF3 from E. coli and associates to the bacterial ribosomes (Lin et al., 1996; Yu and Spremulli, 1997). IF3 does not have a structural homolog among eukaryotic initiation factors. Eukaryotic initiation factor elF1 is considered to be its functional homolog (Lomakin et al., 2006). 
Bacterial IF3 is encoded by infC which contains the unusual start codon AUU (Sacerdot et al., 1982). IF3 regulates the expression of its own gene at the level of translation (Butler et al., 1986) by means of autorepression. This regulation was shown to be dependent on the presence of the AUU start codon and is abolished if AUU is changed for AUG (Butler et al., 1987). It was earlier thought that IF3 is able to discriminate between canonical and non-canonical start codons (Meinnel et al., 1999; Sacerdot et al., 1996; Sussman et al., 1996) but later experiments suggested that IF3 self-regulation is based on following the kinetic effects (Antoun et al., 2006b). Due to the mutual stabilization of the mRNA and the $\mathrm{fMet}^{-t R N A} \mathrm{fMet}^{\mathrm{f}}$ on the $30 \mathrm{~S}$ via codon recognition, the initiation complexes containing the AUU mRNA are less stable than initiation complexes containing classical AUG mRNA. The stability of fMet-tRNA ${ }^{\text {fMet }}$ and mRNA do not depend on the presence of IF3 in the system. One of the known functions of IF3 in the cell is preventing association of the ribosomal subunits. Therefore at higher IF3 concentrations in the cell, the rate of $50 S$ joining the 30S IC decreases and at some point becomes similar to the rate of the AUU mRNA dissociating form the $30 \mathrm{~S}$ IC. Thus, the initiation complexes containing AUU mRNA will not enter translation initiation, whereas the efficiency of initiation on the AUG-containing mRNA will remain high (Antoun et al., 2006b).

The structure of IF3 has been solved by NMR spectroscopy (Garcia et al., 1995a, 1995b; Moreau et al., 1997), X-ray diffraction (Biou et al., 1995) and cryo-EM reconstruction (Julián et al., 2011). IF3 consists of two domains connected by a flexible linker (Fig 1.5) (Fortier et al., 1994; Garcia et al., 1995a, 1995b; Hua and Raleigh, 1998; Julián et al., 2011). The $N$-terminal domain of IF3 has a globular $\alpha / \beta$-fold; the $C$-terminal domain consists of two-layered $\alpha / \beta$ sandwich fold (Garcia et al., 1995a, 1995b). Most of the functions of IF3 can be performed by the C-domain (Garcia et al., 1995b; Petrelli et al., 2001). The N-domain stabilizes IF3 in the complex with the 30 S subunit (Petrelli et al., 2001). The $\mathrm{N}$-domain was showed to be involved in start codon recognition, initiator tRNA selection and inhibition of leaderless mRNA translation (Maar et al., 2008). The linker between the $\mathrm{N}$ - and $\mathrm{C}$ - domains is important for the maintenance of initiation fidelity by IF3; however, variations in its length or composition did not show a considerable change in the IF3 binding to the 30 S subunit (De Cock et al., 1999).

Sites of interaction of IF3 with the 30 S subunit were determined by mutational analysis (Petrelli et al., 2003; Tapprich et al., 1989), NMR (Sette et al., 1999), chemical probing (Dallas and Noller, 2001; Fabbretti et al., 2007) and cryo-EM reconstruction (Julián et al., 2011; McCutcheon et al., 1999). IF3 binds to the platform of the 305 subunit (Dallas and Noller, 2001; Fabbretti et al., 2007; McCutcheon et al., 1999). IF3-CTD is located at the 12 
loop 790 of the 16S rRNA; IF3-NTD binds close to the fMet-tRNA ${ }^{\text {fMet }}$ (Dallas and Noller, 2001; Julián et al., 2011; Tapprich et al., 1989). The mutations in the $m^{2} G 966$ and $\mathrm{m}^{5} \mathrm{C} 967$ residues located in the 970 loop of $16 \mathrm{~S}$ rRNA were shown to affect binding of IF3 to the 30S subunit (Saraiya et al., 2008). This may be either the result of direct interaction of IF3 with the 970 loop of 16S rRNA or an indirect effect of a conformational change on the 30S that affects IF3 binding site (Dallas and Noller, 2001; Fabbretti et al., 2007; Petrelli et al., 2001). There is also a possibility that 970 loop forms a transient contact with IF3 (Saraiya et al., 2008).

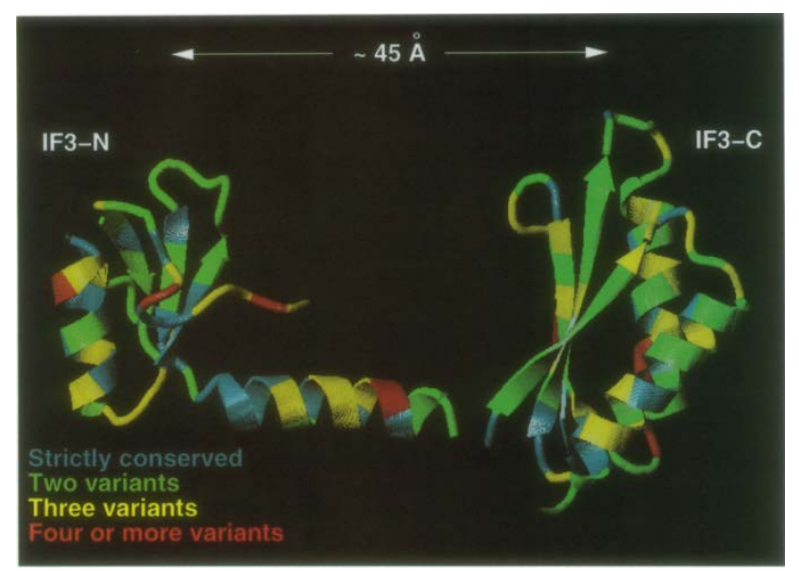

Figure 1.5 (from Biou et al., 1995) A model for the relative disposition of the two halves of IF3. The structures of the C-domain and $\mathrm{N}$ domain were solved separately by X-ray crystallography. The colors show the variation in sequence at each position based on the alignment of sequences.

IF3 has several functions during translation initiation.

1. IF3 prevents ribosomal subunits association by binding to the $30 \mathrm{~S}$ subunit (Antoun et al., 2006a; Debey et al., 1975; Grunberg-Manago et al., 1975). It was initially identified as a ribosomal dissociation factor (Subramanian and Davis, 1970; Subramanian et al., 1968). Once bound, IF3 does not dissociate from the 30S subunit before the arrival of the 50 S subunit (Milon et al., 2008).

2. IF3 ensures the fidelity of the translation initiation complex formation (Antoun et al., 2006b; Hartz et al., 1989, 1990; Milon et al., 2008; Risuleo et al., 1976).

3. IF3 contributes to the accuracy of the initiator tRNA selection by affecting association and dissociation rates of tRNAs from the ribosome (Antoun et al., 2006b; Gualerzi et al., 1977; Wintermeyer and Gualerzi, 1983). Earlier it was thought that IF3 is able to recognize specific motifs of initiator tRNA (Gualerzi and Pon, 1990; Hartz et al., 1989, 1990; Risuleo et al., 1976). Later IF3 was shown to stimulate dissociation of deacylated tRNA (Karimi et al., 1999; Peske et al., 2005) or incorrectly bound tRNAs from the ribosomal P site (Antoun et al., 2006b). 
4. IF3 determines the translation efficiency of a particular mRNA. During transition from the 30 S IC to 70S IC there is a kinetic checkpoint where IF3 discriminates against mRNAs with unfavorable TIR (Grigoriadou et al., 2007b; Milon et al., 2008; La Teana et al., 1993) or non-initiator codon in the P site (Haggerty and Lovett, 1997; Meinnel et al., 1999; Sussman et al., 1996).

Other functions of IF3. IF3 is not only involved in translation initiation but also has other functions. It stimulates the dissociation of deacylated tRNAs form posttermination complexes and helps to dissociate ribosomes into subunits (Hirokawa et al., 2002; Karimi et al., 1999; Peske et al., 2005). IF3 plays a role in recycling of stalled ribosomal complexes (Singh et al., 2005, 2008) and together with IF1, is involved in a cell's response to the cold shock conditions (Giuliodori et al., 2004, 2007).

\section{Initiator tRNA.}

The first amino acid of any protein in all kingdoms of life is methionine; in prokaryotes, the methionine is formylated. During translation it is delivered to the ribosome by the initiator tRNA (tRNA ${ }^{\mathrm{fMet}}$ ) which is capable of recognizing of alternative initiation codons (AUG, GUG, UUG) (Blattner et al., 1997; Kozak, 1999; RajBhandary, 1994). Initiator tRNA is very special in its structure and function as it has to be distinguished from the pool of elongator tRNAs (Rich and RajBhandary, 1976).

Initiator tRNA ${ }^{\text {fMet }}$ possesses a number of unique structural features (Fig 1.6):

1. A mismatch between nucleotides 1:72 (C1:A72 in E. coli) at the end of the acceptor stem; this pair of nucleotides prevents binding of fMet-tRNA ${ }^{\text {fMet }}$ to EF-Tu and is one of the determinants for the formylation of Met attached to the tRNA ${ }^{\text {fMet }}$ (RajBhandary, 1994; Varshney et al., 1993);

2. Base pair 11:24 is purine:pyrymydine respectively, instead of 11:24 pyrymydine:purine in the elongator tRNAs (Varshney et al., 1993);

3. Three conserved consecutive GC base pairs at the bottom of the anticodone stem. These base pairs make the anticodon stem less flexible compared to the anticodon stem loops of elongator tRNAs which favors biding of the tRNA ${ }^{\mathrm{fMet}}$ to the $\mathrm{P}$ site on the ribosome (Cory et al., 1968; Dube et al., 1968; Rich and RajBhandary, 1976; Schweisguth and Moore, 1997; Seong and RajBhandary, 1987). 
Aminoacylation. tRNA ${ }^{\text {fMet }}$ is aminoacylated by methionyl-tRNA synthetase (MetRS). The anticodon is crucial for the recognition of tRNA ${ }^{\text {fMet }}$ by MetRS. Aminoacylation will not take place if the anticodon of initiator tRNA is mutated (Fourmy et al., 1991; Mechulam et al., 1999).

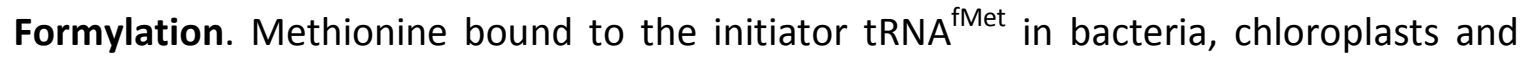
mitochondria is formylated by methionyl-tRNA formyltransferase (MTF) (Schmitt et al., 1996a, 1996b, 1998). This enzyme uses N-10 formyltetrahydrofolate as a donor of formyl group. The non-Watson-Crick base pair 1:72 in tRNA-fMet is a crucial marker by which MTF recognizes initiator tRNA (Schmitt et al., 1998). Formylation is important for initiation of protein synthesis. E. coli strains with disrupted fmt gene coding for MTF show severe growth defects (Guillon et al., 1992; Meinnel et al., 1993; Varshney and RajBhandary, 1992).

The presence of the formyl group is an important determinant which distinguishes initiator from elongator tRNAs (Kozak, 1999; RajBhandary, 1994). The formyl group attached to methionine on the tRNA ${ }^{\text {fMet }}$ favors selection of fMet-tRNA ${ }^{\text {fMet }}$ by IF2 (Sundari et al., 1976) and prevents its binding to EF-Tu (Hansen et al., 1986; Nissen et al., 1995; Seong and RajBhandary, 1987). In most cases formylated methionine is removed from the polypeptide chain after the translation has started (BingelErlenmeyer et al., 2008; Bonissone et al., 2013; Kozak, 1983).
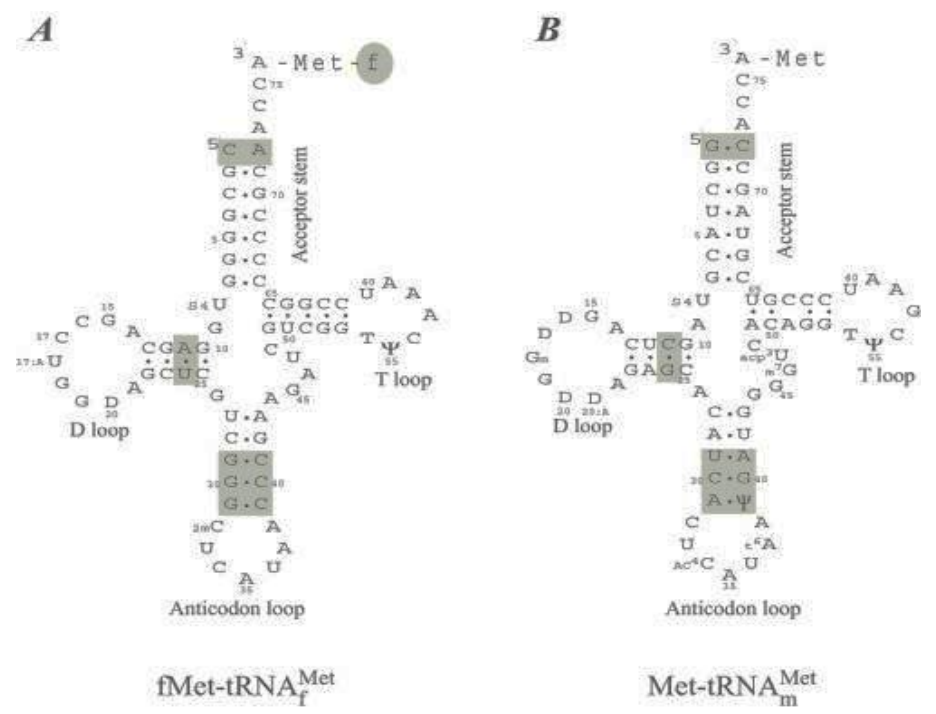

Figure 1.6 (from Laursen et al., 2005) Initiator and elongator methionine-accepting tRNAs. Cloverleaf representation of methionine-accepting tRNAs: (A) initiator tRNA and (B) elongator tRNA. The regions important for initiator tRNA identity are highlighted. See explanation in the text. 
mRNA.

Translation initiation region. Any single-stranded RNA binds to 305 subunits with high affinity even in the absence of initiation factors or fMet-tRNA ${ }^{\text {fMet }}$ (Kozak, 1999; de Smit and van Duin, 2003). The mRNA itself contains a combination of several elements affecting the efficiency of initiation on this mRNA and hence the efficiency of its translation. These elements are located in translation initiation region (TIR) of the mRNA and include the start codon, the Shine-Dalgarno (SD) sequence (complementary to the anti-Shine-Dalgarno (aSD) sequence in 16S rRNA of the ribosome), the spacer between the SD sequence and the start codon, the mRNA secondary structure and the A/U-rich elements in the mRNA which are recognized by protein S1 of the 30S subunit (Dreyfus, 1988; Jin et al., 2006; Milon et al., 2008; Nakamoto, 2009; Steitz, 1969). In bacterial mRNAs TIR includes the region from -20 to +15 nucleotides around the translation start codon (Dreyfus, 1988). The role of TIR is to proper position the mRNA on the 30S subunit for translation initiation.

mRNA secondary structure. Translation initiation region may include some mRNA secondary structure. As in bacteria there are no known RNA helicases involved in this process, the ribosome binds mRNA unstructured regions in the vicinity of the initiator codon and then has to unfold the mRNA and move towards the start codon. The expression of many genes is regulated through the variation of the secondary structure of TIR (de Smit, 1998). Different mRNAs can alter their secondary structure in response to temperature changes (Hoe and Goguen, 1993; Johansson et al., 2002), to the level of cellular protein (Nomura et al., 1984) or to the particular metabolite (Mandal and Breaker, 2004; Mandal et al., 2003; Winkler et al., 2002a, 2002b). Unfolding of TIR further stabilizes mRNA on the ribosome through other interactions (Studer and Joseph, 2006).

Standby site. It was earlier proposed that the ribosome binds any mRNA while the latter exists for a short time in a transient unfolded state. However this scenario is unlikely to happen due to the kinetics of mRNA secondary structure formation (De Smit and Van Duin, 2003). Instead the ribosome binds first to the $A / U$ unstructured regions adjacent to the structured TIR (Scherer et al., 1980; de Smit and van Duin, 2003). These regions are called „standby sites" (Brandt and Gualerzi, 1991; de Smit and van Duin, 2003). The ribosomal protein S1 was shown to bind the standby sites (Boni et al., 1991). Direct interaction between S1 and mRNA was shown by cryo-EM studies (Sengupta et al., 2001). The binding of the $30 \mathrm{~S}$ subunit to the standby site promotes unfolding of TIR 
(Studer and Joseph, 2006) and ribosome goes further into place (De Smit and Van Duin, 2003).

The Shine-Dalgarno sequence. The Shine-Dalgarno sequence is a purine-rich sequence in the $5^{\prime}$-untranslated region of the mRNA consisting of $4-8$ nucleotides. It is separated from the start codon by a 5-13 nucleotide-long spacer (Schneider et al., 1986). A typical SD sequence in $E$. coli is GGAGG and most common spacer length is $7-10$ nucleotides long (Ma et al., 2002). The SD sequence base pairs to the aSD sequence located at the 3 'end of 16S rRNA (Shine and Dalgarno, 1974). SD-aSD interaction stabilizes mRNA on the $30 \mathrm{~S}$ subunit after the primary interaction of the mRNA with the ribosome through the standby sites and unwinding of mRNA secondary structure.

The presence of the SD sequence itself is not essential for the translation and there is little correlation between the strength of SD and translation efficiency (Calogero et al., 1988; Lee et al., 1996; Ma et al., 2002; Melançon et al., 1990; Nakamoto, 2006; de Smit and van Duin, 1994). Sequence analysis of a large number of bacterial genomes showed that SD is present in about $40 \%$ of all mRNAs (Ma et al., 2002). Furthermore, some mRNA do not have a SD sequence or even no 5' UTR. Non-SD mRNAs are found in most bacteria and archaea (Chang et al., 2006; Ma et al., 2002; Tolstrup et al., 2000; Weiner et al., 2000). Leaderless mRNAs are typical for some bacterial phyla, e.g. Actinobacteria, Deinococcus, and Termus (Zheng et al., 2011). Leaderless mRNAs bind directly to the 70 S ribosomes (Grill et al., 2000).

The start codon. The canonical most frequently used start codon is AUG ( $83 \%$ in E. coli) (Ma et al., 2002). Non-canonical start codons are found in a number of genes, e.g. GUG ( $14 \%$ in E. coli), UUG (3\% in E. coli). Rare non-canonical codons AUU, AUC and CUG have been reported (Baudet et al., 2010; Binns and Masters, 2002; McCarthy and Brimacombe, 1994; Polard et al., 1991; Schneider et al., 1986). Curiously, having AUG for the start codon may correlate with the presence of SD in mRNAs (Ma et al., 2002).

The model for mRNA binding. The current model of mRNA binding to the 30S subunit proposes that there are 2 steps in binding: an initial binding step through the standby sites on the 30S subunit (Marzi et al., 2007; de Smit and van Duin, 2003) followed by mRNA stabilization on the 30S IC (Milon et al., 2008). Most likely, all mRNAs that lack very extensive secondary structure at the translation initiation region can bind to the 30S subunit with similar efficiencies. The stability of mRNA binding on the 30S subunit depends on the regulatory elements in MRNA TIR and the presence of initiation factors in the initiation complex (Nakamoto, 2009). 
mRNA is stabilized on the 30S subunit by SD-aSD interactions which do not depend on the presence of initiation factors. This interaction helps further accommodation of the AUG codon in the P site (Kaminishi et al., 2007; La Teana et al., 1995). The start codon recognition by the $\mathrm{fMet}_{\mathrm{ARNA}} \mathrm{fMet}^{\mathrm{M}}$ makes the complex even more stable causing the conformational change of the $30 \mathrm{~S}$ subunit that leads to IF3 destabilization and 50S subunit binding (Milon et al., 2008).

\subsection{S PIC formation.}

The primary binding of initiation factors to the 30S subunit is independent on each other. Therefore it was earlier assumed that initiation factors bind the ribosome in a random order so that a number of alternative binding pathways exist (Gualerzi and Pon, 1990). Recent data suggests that there is a kinetically favored order of initiation factors binding to the ribosome (Fig 1.7) (Milón et al., 2012a).

IF3 binding. After translation termination, ribosomes have to be split into the subunits to re-enter the next round of initiation. Ribosome recycling factors RRF and EF-G split the post-termination complexes consisting of the ribosome, the mRNA with the termination codon in the A site and deacylated tRNA in the P site into 30S-mRNA-tRNA complex and 50S subunit (Karimi et al., 1999; Peske et al., 2005). IF3 prevents premature reassociation of $50 \mathrm{~S}$ subunits to the $30 \mathrm{~S}$ (Peske et al., 2005). The arrival time of IF3 to the $30 \mathrm{~S}$ is $<1 \mathrm{~ms}$. IF3 was shown to bind with a similar rate to the 30S-mRNA-tRNA complex and to the vacant 30S (Milón et al., 2012b). In the first case IF3 promotes dissociation of mRNA and deacylated tRNA from the complex and the association of the new mRNA is delayed (Karimi et al., 1999; Peske et al., 2005). The binding of IF3 to the small ribosomal subunit is biphasic. During the first step, IF3 associates rapidly with the 30S. At this step the binding is not influenced by the presence of two other factors on the ribosome. Subsequently, upon the rearrangement in the complex, IF3 is stabilized. The presence of IF1 enhances IF3 stabilization on the 30S. The formation of the complete $30 \mathrm{~S} \mathrm{IC}$, containing $\mathrm{FMet}^{-\mathrm{RRNA}}{ }^{\mathrm{fMet}}$, mRNA and all three initiation factors, led to rapid dissociation of IF3 form the complex (Milón et al., 2012b). \#

IF2 binding. IF2 joins the $30 \mathrm{~S}$ subunit very rapidly with the arrival time of about $1.3 \mathrm{~ms}$. To free 30 S subunits IF2 is recruited approximately at the same time as IF3. IF2 binding to the $30 \mathrm{~S}$ does not depend on the presence of IF3. The complex 30S-IF2-IF3 is short- 
lived and can dissociate rapidly. The addition of mRNA does not affect IF2 stability in the complex. However addition of IF1 and/or fMet-tRNA ${ }^{\text {fMet }}$ leads to the strong IF2 stabilization in the 30S PIC (Milón et al., 2012a).

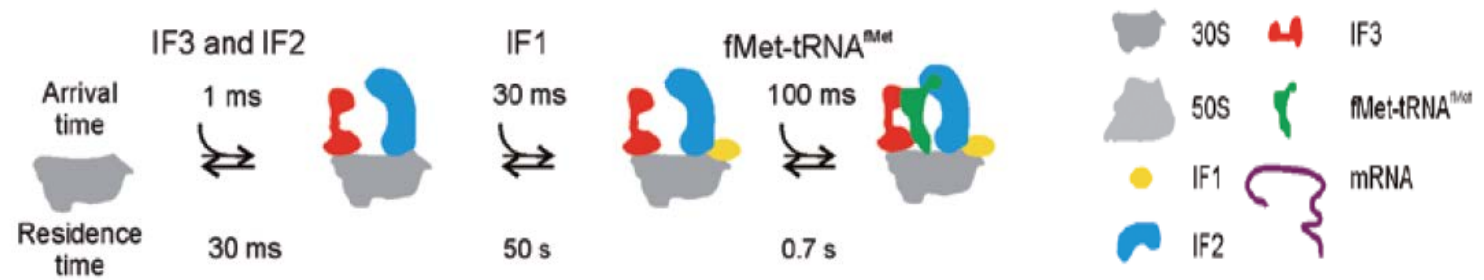

Figure 1.7 (from Milon and Rodnina, 2012) Elemental steps of 30S PIC assembly on the vacant $30 S$ subunits. The kinetically favored order of initiation factors and fMet-tRNA ${ }^{\text {fMet }}$ arrivals and resident times of the respective complexes are indicated. See explanations in the text.

IF1 binding. IF1 is the last of initiation factors to arrive to the $30 \mathrm{~S}$ PIC after $30 \mathrm{~ms}$. The complexes containing 30S-IF1-IF2 or 30S-IF1-IF3 were shown to dissociate readily with dissociation rate constants of $2 \mathrm{~s}^{-1}$ and $0.7 \mathrm{~s}^{-1}$. The presence of both IF2 and IF3 in the 30 SIC leads to IF1 stabilization in the complex with the dissociation rate constant of $0.02 \mathrm{~s}^{-1}$. This effect is reciprocal - the presence of IF1 also stabilizes the already bound IF2 and IF3. The presence of FMet-tRNA ${ }^{\text {fMet }}$ and mRNA with AUG codon leads to even greater IF1 stabilization on the 30S PIC with ten-fold lower dissociation rate constant of $0.0025 \mathrm{~s}^{-1}$ (Milón et al., 2012a).

Interplay between the factors. Although the binding of all the factors is independent of each other, there is interplay between the factors on the ribosome. The binding of each factor to the ribosome depends on the presence of the other two factors. The affinity of IF1 to the 30S subunit is increased when IF2 or IF3 are already present (Milón et al., 2012a; Zucker and Hershey, 1986). If both IF2 and IF3 are present, the affinity of IF1 to the $30 \mathrm{~S}$ subunit is increased further, more than 35-fold (Zucker and Hershey, 1986). In turn, the presence of IF1 stabilizes IF2 or IF3 (10-fold) binding to the 30 S subunit (Milón et al., 2012a). Stabilization of IF3 by IF2 (4-fold) has also been also observed (Milón et al., 2012a).

Rearrangements of the 30S PIC. Binding of the initiation factors causes conformational rearrangements in the 30S PIC. Due to these rearrangements mutual stabilization of the initiation factors on the ribosome is possible. Flexibility of the $30 \mathrm{~S}$ ribosomal subunit is 
important for the intermolecular communication in the complex. Cryo-EM reconstructions of the $30 \mathrm{~S}$ IC revealed that binding of the initiation factors and fMettRNA $^{\text {fMet }}$ induce a rotation of the head relative to the body of the $30 \mathrm{~S}$ subunit (Julián et al., 2011). In the absence of IF3, 30S was seen in the classical non-rotated state (Simonetti et al. 2009), whereas in the presence of IF3 30S 70S is observed in the rotated state with the head tilted towards the platform (Julián et al., 2011). The 30 S remains in rotated conformation upon $50 \mathrm{~S}$ subunit joining until GTP hydrolysis by IF2 and the dissociation of the factor (Marshall et al., 2009).

IF1 and IF3. Binding sites of IF1 and IF3 are not close to one another and factors bind the ribosome in an independent manner. However IF3 undergoes a conformational rearrangement $\left(k_{\mathrm{app}}=55 \mathrm{~s}^{-1}\right)$ after its initial biding to the ribosome at a rate similar to IF1 binding $\left(k_{o n}=20 \mu \mathrm{M}^{-1} \mathrm{~s}^{-1}\right.$ if IF3is already present in the complex). Also IF3 binding causes conformational change in 30S ribosomal subunit - the movement of the head of the $30 \mathrm{~S}$ relative to the body (Julián et al., 2011). This change creates a conformational landscape which accelerates IF1 binding.

IF1 is known to induce distortions in the helix 44 of 165 rRNA disrupting the A1413:G1487 base pair (Carter et al., 2001). When the dynamics of the 30S was impaired using antibiotic streptomycin, communication between IF1 and IF3 was impaired (Milon et al., 2008). Mutations in this region of 16S rRNA or in the respective regions of IF1 and IF3 disrupted communication between the factors and, as a consequence, fidelity of initiation was affected (Milon et al., 2008; Qin and Fredrick, 2009; Qin et al., 2007).

IF1 and IF2. Dissociation of IF2 from the 30S PIC is slowed down by about 10-fold in the presence of IF1 (Milón et al., 2012a). This occurs due to the rearrangement of IF2 induced by the interaction with IF1. However, the stabilization may be characteristic for E. coli as its IF2 has an extended NTD that forms a contact to IF1 on the 30 S subunit. The IF2 NTD of the thermophilic organisms is shorter and does not interact with IF1 (Kapralou et al., 2008).

IF2 and IF3. IF3 is also thought to induce a conformational change of IF2 which is required for IF1 stabilization on the $30 \mathrm{~S}$ PIC as there is no direct interaction between IF1 and IF3, but IF1 and IF2 interact via IF2 NTD (Julián et al., 2011). Comparison of the cryoEM reconstruction of the 30 S IC with and without IF3 also reveals that IF2 CTD is positioned in a different way depending on IF3 presence (Julián et al., 2011; Simonetti et 
al., 2009). Thus, IF3-induced conformation of IF2 may also have a role in modulating subsequent fMet-tRNA ${ }^{\text {fMet }}$ binding.

\section{mRNA binding.}

The amount of mRNA in the cell changes depending on the growth stage or in response to the changing conditions. Concentration of mRNAs in the cell may vary by orders of magnitude and not all mRNAs in the cell are expressed at the same time (McCarthy and Gualerzi, 1990; Passalacqua et al., 2009). Whether the particular mRNA is going to be translated is decided at the level of translation initiation, namely at the steps of 30S PIC assembly, its rearrangement to the 30S IC and 70S IC formation (Antoun et al., 2006a; Grigoriadou et al., 2007a; Milon et al., 2008; Studer and Joseph, 2006).

The efficiency of mRNA incorporation into the 30S PIC depends on two factors: the efficiency of initial mRNA binding to the complex and its further stabilization in the complex (Kudla et al., 2009; McCarthy and Gualerzi, 1990; Milón et al., 2012b; Studer and Joseph, 2006). The primary mRNA binding has a strong correlation to the abundance of a given mRNA type in the cell (Passalacqua et al., 2009) and the availability of A-U-rich sequences for the contact with the protein S1 (Komarova et al., 2002; Skorski et al., 2006). Not all of the mRNAs that had a contact with the 30S PIC will enter translation. Stabilization of mRNA on the 30S PIC depends on its TIR secondary structure and the presence of the regulatory elements (Milón et al., 2012b; Studer and Joseph, 2006).

Initial docking of mRNA is facilitated by the A-U-rich regions of the mRNA which are recognized by ribosomal protein S1 (Komarova et al., 2002). S1 gene is essential for growth in E. coli (Kitakawa and Isono, 1982; Sørensen et al., 1998). S1 facilitates primary binding of mRNA to the ribosome and possibly assists in unfolding mRNA secondary structures in the TIR region needed for further mRNA stabilization. S1 is important for initial mRNA selection (Ringquist et al., 1995). It was shown to act synergistically with SD: the stronger SD of the mRNA, the more pronounced stimulating effect of S1 (Vimberg et al., 2007).

After the primary binding of mRNA to the 30S subunit, secondary structure of TIR has to be unfolded to make available the SD sequence and the start codon (Marzi et al., 2007). The secondary structure is an important determinant of the efficiency of translation initiation as altering the GC contents in the TIR region can significantly reduce 
translation of a particular mRNA (Allert et al., 2010; Kudla et al., 2009; Voges et al., 2004).

When TIR is unwound, the SD sequence makes contact to the aSD sequence on the 16S rRNA. This interaction is thought to stabilize mRNA on the ribosome. However SD-aSD interaction is not an absolute requirement for translation (Calogero et al., 1988; Melançon et al., 1990). In some cases extended SD-aSD complementarity, in combination with a short spacer between the SD sequence and the start codon, impairs translation on a given mRNA (Komarova et al., 2002; Lee et al., 1996; Skorski et al., 2006; de Smit and van Duin, 1994). On the other hand, translation efficiency of a particular mRNA does not necessarily correlate with the presence of SD (Calogero et al., 1988; Lang et al., 1989).

Cryo-EM reconstructions suggested that mRNA binds an mRNA docking site on the ribosome, which is located at the platform of the 30 S subunit (Marzi et al., 2007). Different structured mRNAs can bind the same region on the ribosome (Allen et al., 2005; Jenner et al., 2005; Yusupova et al., 2006). The mRNA docking site shows no sequence specificity in mRNA binding but rather ribosomal proteins in the vicinity provide positive charges for binding of any mRNA type (Marzi et al., 2007). The proteins that form the mRNA docking site on the platform of the 30 S subunit are S1, S2, S7, S11, S18, S21 and S1 (Allen et al., 2005; Jenner et al., 2005; Marzi et al., 2007; Sengupta et al., 2001).

The position of mRNA on the ribosome changes with time from the mRNA primary binding until mRNA accommodation in the initiation site (Canonaco et al., 1989; RinkeAppel et al., 1994; La Teana et al., 1995; Yusupova et al., 2006). First, the mRNA is recruited to the platform of the $30 \mathrm{~S}$ and orients towards the S2 protein. Then, the SDaSD interaction is formed. During the next step initiation codon of the mRNA is positioned to the into the ribosomal $\mathrm{P}$ site where the start codon recognition takes place. At this step SD also moves towards the protein S18. As a result of all these rearrangements the correct reading frame is established and the mRNA is ready for translation (Yusupova et al., 2006). 


\section{fMet-tRNA $^{\text {fMet }}$ binding to the 30 S subunit.}

It was originally proposed that IF2 acts as a carrier for the initiator tRNA and delivers it to the ribosome forming a ternary complex in a manner similar to other translational GTPases like EF-Tu, SelB or eIF2 (Wu and RajBhandary, 1997). IF2 has some features in common with EF-Tu, which is a known tRNA carrier. Both factors show some sequence homology in their $\mathrm{G}$ domains and G3 (domain 2 in EF-Tu) and both are GTP-binding proteins. However, there was evidence against IF2 being a tRNA carrier showing that IF2 stimulated the binding of AcPhe-tRNA ${ }^{\text {Phe }}$ to poly(U)-programmed ribosomes, which meant that IF2 binds the 30S prior tRNA binding (Wintermeyer and Gualerzi, 1983).

There are only two possibilities how fMet-tRNA ${ }^{\text {fMet }}$ can bind 30S PIC. Either fMettRNA ${ }^{\text {fMet }}$ binds to 30 S PIC directly or it is delivered as a part of a ternary complex with IF2-GTP. The way of FMet-tRNA $^{\text {fMet }}$ binding to the 30 S subunit was investigated by Milon et al. using rapid kinetic techniques (Milon et al., 2010). They found that the ternary complex formed in solution between fMet-tRNA ${ }^{\text {fMet }}$-IF2-GTP was weak ( $\left.K_{d}=1 \mu M\right)$ and easily dissociated. At the same time, in the absence of fMet-tRNA $^{\text {fMet }}$, IF2-GTP was binding to the $30 \mathrm{~S}$ PIC rapidly and tightly possibly causing conformational rearrangement in the subunit (Caserta et al., 2006; Milon et al., 2010). The rate of IF2 binding to the $30 \mathrm{~S}$ PIC was approximately 40 times higher than the rate of fMet-tRNA ${ }^{\text {fMet }}$ biding; and the rate of $\mathrm{fMet-tRNA}{ }^{\mathrm{fMet}}$ binding to the ribosome was not altered by binding fMet-tRNA $^{\text {fMet }}$ alone or in the complex with IF2-GTP. Taken together, these results suggest that IF2 binds 30S PIC prior to fMet-tRNA ${ }^{\text {fMet }}$. Binding of fMet-tRNA ${ }^{\mathrm{fMet}}$ is the slowest reaction during the PIC assembly (Milon et al., 2010). Most likely, the binding occurs in two steps with a fast bimolecular binding step and a slower step of tRNA adjustment on the 30 subunit (Milon et al., 2010).

fMet-tRNA $^{\text {fMet }}$ is stabilized in the PIC as IF2 provides important anchoring points for the initiator tRNA and possibly induces the conformation of the 30S subunit which favors fMet-tRNA $^{\text {fMet }}$ binding (Canonaco et al., 1986; Caserta et al., 2006; Milon et al., 2010). Site directed mutagenesis and NMR spectroscopy suggested that C2 domain of IF2 interacts with aminoacylated and formylated acceptor arm of the initiator tRNA and with N-formyl-methionine (Guenneugues et al., 2000). The interaction between fMet and IF2 was suggested to induce the conformational change in the anticodon stem of the tRNA (Mayer et al., 2003). The amino acid attached to the tRNA ${ }^{\text {fMet }}$ is less important for its recognition by IF2 than the formylation as IF2 is capable of discrimination for aminoacyl-tRNAs that have their aNH2-group blocked (Antoun et al., 2006b; Boelens 
and Gualerzi, 2002; Canonaco et al., 1986). For example, IF2 binds fVal-tRNA and fGIntRNA but does not bind unformylated Met-tRNA ${ }^{\text {fMet }}$ (Wu and RajBhandary, 1997). IF2 overexpression makes initiation possible with non-formylated Met-tRNA ${ }^{\text {fMet }}$ (Guillon et al., 1996; Wintermeyer and Gualerzi, 1983).

\subsection{Transition to the 30S IC. Start codon recognition.}

The most important event during translation initiation is the selection of the correct reading frame on the mRNA. This is achieved upon mRNA start codon recognition by the anticodon of $f M e t-t R N A^{f M e t}$ in the $P$ site on the ribosome. Start codon recognition is an important point of affinity switch when $30 \mathrm{~S}$ PIC is transformed into mature $30 \mathrm{~S} \mathrm{IC,} \mathrm{which}$ determines its following transition to the 70S IC. Formation of the SD-aSD interaction is sufficient to place start codon of the SD-led mRNA to the P site (Gold, 1988). However initiation on the non-SD-led mRNAs also has high fidelity, suggesting that the SD-aSD interaction is not essential for the proper start codon recognition (Calogero et al., 1988) implying that the ribosome has different means of controlling the codon-anticodon interaction.

The initiation factors have an important role in monitoring the correct start codon recognition and checking the quality of the 30 S IC. IF3 is known to increase the turnover of any tRNA on and off the ribosome and the presence of IF1 in the complex enhances this effect (Antoun et al., 2006b; Wintermeyer and Gualerzi, 1983). At this point the positive discrimination for the initiator tRNA is made. One of the unique features of the initiator tRNA is the three GC base pairs in the anticodon stem. Two of these base pairs (G29-C41 and G30-C40) interact with 16S rRNA residues G1338 and A1339, respectively (Mandal et al., 1996; Qin et al., 2007). This interaction increases the stability of fMettRNA $^{\text {fMet }}$ on the ribosome compared to elongator tRNAs. Upon start codon recognition, IF3 is destabilized and dissociates from the ribosome (Milon et al., 2008; Milón et al., $2012 b$ ). If the proper codon recognition took place, fMet-tRNA ${ }^{f M e t}$ is locked in the complex on the start codon of the mRNA. If there is no proper codon-anticodon interaction in the complex, 30S PIC will release the 50S and will not proceed to the 70S IC formation (Milon et al., 2008). 


\subsection{Formation of the 70S IC.}

The formation of the 70S IC is the next step of translation initiation (Fig. 1.8). It starts with the 50S subunit binding to the $30 \mathrm{~S} \mathrm{IC} \mathrm{carrying} \mathrm{three} \mathrm{initiation} \mathrm{factors,} \mathrm{fMet-tRNA}{ }^{\text {fMet }}$ and mRNA (Grigoriadou et al., 2007a; Milon et al., 2008). 30S-bound IF2 in complex with GTP promotes rapid subunit association (Antoun et al., 2006a; Milon et al., 2008). The subunit joining was not influenced by absence of IF2-catalyzed GTP hydrolysis when GTP was substituted for its non-hydrolysable analog GDPNP (Antoun et al., 2003; Marshall et al., 2009). Being bound to the ribosome, IF2 together with fMet-tRNA ${ }^{\text {fMet }}$ provide a large docking surface for the 50S subunit helping to overcome the electrostatic barrier (Julián et al., 2011; Marshall et al., 2009). Rapid subunit association depends on the interaction of IF2 with the protein L12 on the 50S subunit (Huang et al., 2010; Mandava et al., 2012). Joining of the 50S subunit most likely occurs in several steps (Fabbretti et al., 2007; Grigoriadou et al., 2007a; Milon et al., 2008). It was shown by time-resolved chemical probing that intersubunit bridges are formed in a sequential manner upon subunits joining (Hennelly et al., 2005). The stepwise nature of 50S docking explains the presence of IF3 in the 70S IC (Allen et al., 2005), although the position of IF3 on the ribosome creates a steric clash for the formation of an important intersubunit bridge $2 b$ (Dallas and Noller, 2001; Julián et al., 2011; McCutcheon et al., 1999). The adjustment of the 50S subunit was shown to happen prior to the final ejection of IF3 from the complex (Fabbretti et al., 2007; Milon et al., 2008). Binding of fMet-tRNA ${ }^{\text {fMet }}$ to the $30 S \mathrm{IC}$ and the start codon recognition lower the affinity of IF3 to the ribosome, but the factor remains bound to the 30 S subunit (Milon et al., 2008). When the 50S subunit docks, it prevents IF3 from rebinding at the interface side of the 30S subunit (Dallas and Noller, 2001; McCutcheon et al., 1999). The docking of the 50S subunit is slower in the presence of IF3 in the complex (Antoun et al., 2006a; Milon et al., 2008), but IF3 is required for control of fidelity of translation initiation on a given mRNA as it is involved in the TIR selection (Milon et al., 2008).

The docking of the 50S subunit to the 30 S IC triggers IF2 GTPase (Grigoriadou et al., 2007a; Huang et al., 2010; Tomsic et al., 2000). The IF2-catalyzed GTP hydrolysis triggers conformational changes of the factor itself as well as the entire ribosome (Allen et al., 2005; Myasnikov et al., 2005). In its GTP-bound form, IF2 interacts mostly with the $30 \mathrm{~S}$ subunit and forms a contact with the fMet-tRNA ${ }^{\text {fMet }}$. After GTP hydrolysis, IF2 accepts socalled "ready-to-leave" conformation moving away from the fMet-tRAN" fMet and partially losing its contacts with the ribosome (Myasnikov et al., 2005). The ribosomal conformation upon GTP hydrolysis changes from the rotated to non-rotated state 
(Marshall et al., 2009). Dissociation of IF2 from the ribosome after GTP hydrolysis and Pi release is the final step of initiation committing the ribosome to translation.

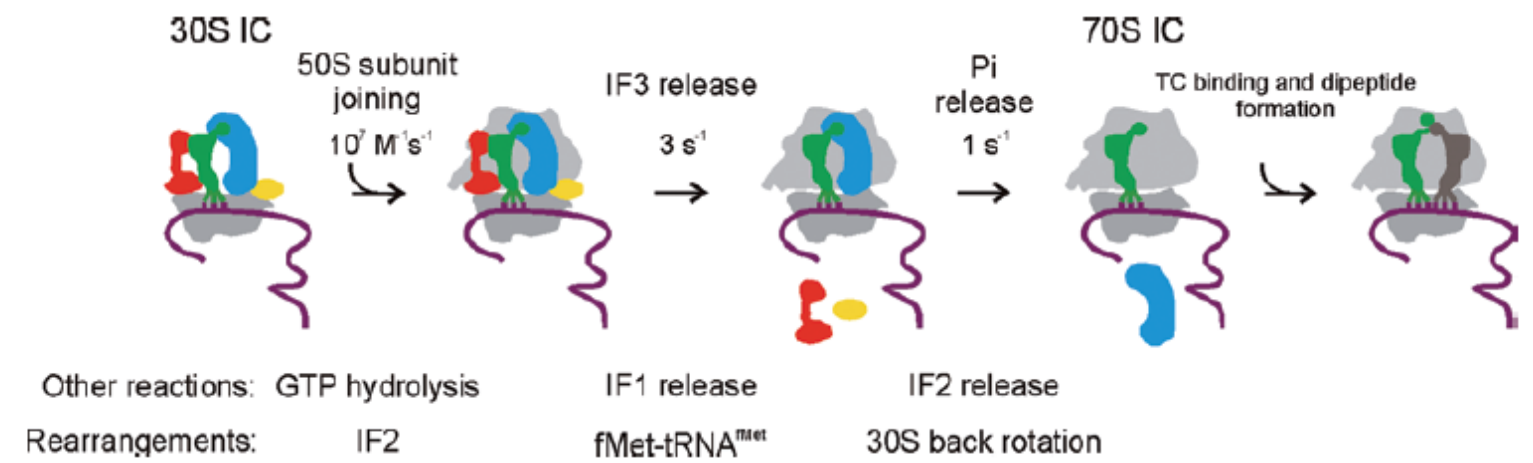

Figure 1.8 (from Milon and Rodnina, 2012) Formation of the 70S IC. Step 1, docking of the $50 \mathrm{~S}$ subunit to the $30 \mathrm{~S} \mathrm{IC} \mathrm{containing} \mathrm{all} \mathrm{three} \mathrm{initiation} \mathrm{factors.} \mathrm{Joining} \mathrm{of} \mathrm{the} 50 \mathrm{~S}$ subunit triggers GTP hydrolysis by IF2, which promotes conformational rearrangements of the factor. Step 2, dissociation of IF3 and IF1. The position of fMet-tRNA ${ }^{\text {fMet }}$ likely changes. Step 3, release of Pi from IF2, dissociation of IF2, and the rearrangements of the 30 S subunit from the rotated to nonrotated state, resulting in a mature 70S IC. The final step, binding of the ternary complex (TC) EFTu -GTP-a-tRNA is followed by the formation of the first peptide bond, committing the complex for elongation. 


\section{Chapter 2. Kinetics of mRNA binding to the 30S IC and 30S PIC.}

\section{Introduction.}

Early events on translation initiation include initiation factors, mRNA and fMet-tRNA ${ }^{\text {fMet }}$ assembly on the 30S ribosomal subunit thereby forming the 30S IC (Gualerzi et al., 2001). After the start codon recognition and 50S subunit joining, the $30 \mathrm{SIC}$ is converted to the 70S IC. The late events of translation initiation comprise GTP hydrolysis by IF2, fMet-tRNA $^{\text {fMet }}$ adjustment in the $P$ site of the ribosome and IF2 dissociation form the ribosome, thus making the 70S IC able to progress to elongation. Although translation initiation has been extensively studied, the studies mostly focused on the late events of translation initiation (Antoun et al., 2006a; Grigoriadou et al., 2007a; Marshall et al., 2009; Milon et al., 2008; Tomsic et al., 2000). Early events in translation initiation have received less attention and, until recently, dynamics of 30S PIC formation was unknown. In a study of 2012 by Milon et al., the dynamics of the 30S PIC formation was monitored using fluorescence resonance energy transfer (FRET) between ligands in the 30S PIC (Milón et al., 2012b). The study revealed the kinetically favored order of initiation factors binding to the 30 S subunit and suggested a kinetic model of the 30S PIC assembly (Milón et al., 2012b).

Binding of mRNA to the $30 \mathrm{~S}$ subunit is independent on the presence of IFs or fMettRNA $^{\text {fMet }}$ on the 30S (Calogero et al., 1988; Milón et al., 2012b; Studer and Joseph, 2006), and vice versa, binding of all three initiation factors and initiator tRNA to the ribosome does not depend on the presence of mRNA (Gualerzi et al. 2001). Based on the structural work, mRNA was suggested to bind to the conserved pre-initiation site on the platform of the 30S subunit where mRNA forms a number of interactions with the ribosome (Marzi et al., 2007; Yusupova et al., 2006). mRNA is thought to interact with the ribosome in two steps (Studer and Joseph, 2006). The initial binding of mRNA to the ribosomal site requires single-stranded regions of the mRNA. Double-stranded mRNA without single-stranded regions is not able of binding $30 \mathrm{~S}$ even when IFs and/or fMettRNA $^{\text {fMet }}$ are present (Studer and Joseph, 2006). The influence of mRNA secondary structure on mRNA binding to the ribosome was studied by Studer and Josef (Studer and Joseph, 2006). A strong correlation was observed between the stability of the mRNA structure and its association and dissociation rate constants (Studer and Joseph, 2006). After binding to the ribosome, mRNA secondary structure unfolds and TIR becomes 
exposed. The SD sequence is capable of interacting with the aSD sequence of $16 S$ rRNA. The position of mRNA on the ribosome is slightly changed due to this interaction. mRNA is stabilized in the 30S PIC complex by SD-aSD interactions (De Smit and Van Duin, 2003; Yusupova et al., 2006).

The efficiency of the first step of mRNA binding to the ribosome depends on mRNA concentration in the cell. In principle, any mRNA that has a single-stranded region shows fast association with the ribosome. So seemingly the more abundant mRNAs will be translated more frequently. However, there exists a kinetic checkpoint after the primary mRNA binding to the $30 \mathrm{~S}$ subunit where mRNAs with favorable TIR structure have the chance to be stabilized in the 30S PIC via SD-aSD interaction and start codon recognition. These type of mRNAs will show slow dissociation from the PIC and are more likely to enter translation (Kudla et al., 2009; McCarthy and Gualerzi, 1990).

TIR was shown to play a role in mRNA stabilization on the 30S PIC when the MRNA is already bound (Studer and Joseph, 2006). However, it was previously unclear whether TIR has any impact on the primary mRNA interaction with the ribosome. It was also unknown whether the initiation factors that are already bound to the ribosome favor the subsequent mRNA selection by TIR. The initiation factors trigger conformational rearrangements of the $30 \mathrm{~S}$ subunit which, in turn, may favor binding of mRNA with particular TIR structure (Studer and Joseph, 2006; La Teana et al., 1995).

The aim of this study was to measure kinetic parameters of the primary binding of mRNA with different TIR to the 30S subunit. We also investigated the influence of initiation factors and fMet-tRNA ${ }^{\text {fMet }}$ on the mRNA binding.

\section{Materials and methods.}

mRNA.

Synthetic mRNAs were used in the study (IBA, Göttingen, Germany). All mRNAs were labeled at the 3'-end with Atto488 (ATTO-TEC GmbH, Siegen, Germany). The nomenclature and sequence of the mRNAs is shown below with the SD sequence shown in red and the start codon is shown in blue. Secondary structures were predicted by Mfold and are shown in Supplementary materials (Supplementary Figure 1.). Melting temperatures and Gibbs free energies of folding are summarized in Supplementary table 1. 
Table 2.1. The nomenclature of the model mRNAs. SD sequence is shown in red and start codon is shown in blue.

\begin{tabular}{|l|l|}
\hline m022 AUG & uuaacagguauacauacuauguucacgauc \\
\hline m002 AUG & cacuuaaggagguauacuauguucacgauc \\
\hline m002 no AUG & cacuuaaggagguauacugguuucacgauc \\
\hline m003 AUG & uuaaucccuucuuauacuauguucacgauc \\
\hline m003 no AUG & uuaaucccuucuuauacuaccuucacgauc \\
\hline m6a & cacuuaaggagacagaaauguuuccuauc \\
\hline
\end{tabular}

\section{Kinetic experiments.}

Kinetic experiments were performed using the SX-20MV stopped-flow apparatus (Applied Photophysics, Leatherhead, UK). Experiments were carried out by rapidly mixing equal volumes ( $60 \mu \mathrm{l}$ each) of reactants at $20^{\circ} \mathrm{C}$, containing typically $30 \mathrm{~S}, 30 \mathrm{~S} \mathrm{PIC}$, $30 S$ IC and different mRNAs. All binding and dissociation reactions were performed in TAKM $_{7}$ buffer: $50 \mathrm{mM}$ Tris $\mathrm{pH}$ 7.5, $70 \mathrm{mM} \mathrm{NH}_{4} \mathrm{Cl}, 30 \mathrm{mM} \mathrm{KCl}, 7 \mathrm{mM} \mathrm{MgCl}_{2}$. All reactions were performed at $20^{\circ} \mathrm{C}$ unless otherwise stated. Atto 488 was excited at $465 \mathrm{~nm}$ and the fluorescence change upon binding of fluorescently labeled mRNA to the 30S subunit monitored using a cut-off filter for $500 \mathrm{~nm}$. The average of 7-10 time courses was taken to decrease the fluorescent signal to noise ratio. The apparent rate constant was determined by fitting the traces with single exponential equation $\mathrm{Y}=\mathrm{A} 0+\mathrm{A} 1^{*} \exp \left(-\mathrm{k} 1^{*} \mathrm{t}\right)$ or double exponential equation $\mathrm{Y}=\mathrm{A} 0+\mathrm{A} 1 * \exp (-\mathrm{k} 1 * \mathrm{t})+\mathrm{A} 2 * \exp (-\mathrm{k} 2 * \mathrm{t})$ where $\mathrm{A} 0$ corresponds to the final amplitude, $A 1, A 2$ to amplitudes of the corresponding phases and $k 1, k 2$ to the respective apparent rates.

\section{Binding experiments.}

mRNA binding to the 30S subunit. Mix 1 contained fixed concentration of fluorescent mRNA-Atto488 (0.05 $\mu \mathrm{M})$ which was the limiting component for the reaction. Mix 2 contained varied concentrations of 30 s ribosomal subunit $(0.15 \mu \mathrm{M} ; 0.2 \mu \mathrm{M} ; 0.3 \mu \mathrm{M} ; 0.4$ $\mu \mathrm{M} ; 0.5 \mu \mathrm{M})$ in excess to the mRNA. 
mRNA binding to the 30S PIC. Mix 1 contained mRNA-Atto488 (0.05 $\mu \mathrm{M})$. Mix 2 contained 30S PIC (0.15 $\mu \mathrm{M}$ 30S; $0.3 \mu \mathrm{M}$ IF1; $0.3 \mu \mathrm{M}$ IF2; $0.3 \mu \mathrm{M}$ IF3; $250 \mu \mathrm{M}$ GTP). Mix 2 was incubated at $37^{\circ} \mathrm{C}$ for 30 min and kept on ice for 10 min before mixing with mRNA.

mRNA binding to the 30S IC. Mix 1 contained mRNA-Atto488 (0.05 $\mu \mathrm{M})$. Mix 2 contained 30S IC (0.3 $\mu \mathrm{M}$ 30S; $0.6 \mu \mathrm{M}$ IF1; $0.6 \mu \mathrm{M}$ IF2; $0.6 \mu \mathrm{M}$ IF3; $0.6 \mu \mathrm{M}$ fMet-tRNA $^{\text {fMet; }}$; $250 \mu \mathrm{M}$ GTP). Mix 2 was incubated at $37^{\circ} \mathrm{C}$ for $30 \mathrm{~min}$ and kept on ice for $10 \mathrm{~min}$ before mixing with mRNA.

\section{Dissocation experiments.}

mRNA dissociation from the 30S. Mix 1 contained $30 \mathrm{~S}(0.15 \mu \mathrm{M})$ and fluorescent mRNA $(0.05 \mu \mathrm{M})$. Mix 1 was incubated for $37^{\circ} \mathrm{C} 30 \mathrm{~min}$ and then kept on ice for $10 \mathrm{~min}$. Mix 2 contained non-fluorescent mRNA $(0.5 \mu \mathrm{M})$ of the same type as one in complex with $30 \mathrm{~S}$.

mRNA dissociation from 30S PIC. Mix 1 contained 30S IC (0.3 $\mu \mathrm{M}$ 30S; $0.6 \mu \mathrm{M}$ IF1; 0.6 $\mu \mathrm{M}$ IF2; $0.6 \mu \mathrm{M}$ IF3; $0.05 \mu \mathrm{M}$ mRNA-Atto 488; $250 \mu \mathrm{M}$ GTP). Mix 1 was incubated at $37^{\circ} \mathrm{C}$ for $30 \mathrm{~min}$ and kept on ice for 10 min before mixing with mRNA. Mix 2 contained nonfluorescent mRNA $(0.5 \mu \mathrm{M})$ of the same type as one in complex with $30 \mathrm{~S}$.

mRNA dissociation from the 30S IC. Mix 1 contained 30S IC (0.3 $\mu \mathrm{M} 30 \mathrm{~S} ; 0.6 \mu \mathrm{M}$ IF1; 0.6 $\mu \mathrm{M}$ IF2; $0.6 \mu \mathrm{M}$ IF3; $0.6 \mu \mathrm{M}$ fMet-tRNA ${ }^{\text {fMet }}$; $0.05 \mu \mathrm{M}$ mRNA-Atto 488; $250 \mu \mathrm{M}$ GTP). Mix 1 was incubated at $37^{\circ} \mathrm{C}$ for $30 \mathrm{~min}$ and kept on ice for $10 \mathrm{~min}$ before mixing with mRNA. Mix 2 contained non-fluorescent mRNA $(0.5 \mu \mathrm{M})$ of the same type as one in complex with 30 S.

\section{Renaturation of mRNAs}

All mRNAs were kept in deionized water at $-20^{\circ} \mathrm{C}$. For renaturation after thawing, mRNAs were incubated at $98^{\circ} \mathrm{C}$ for $2 \mathrm{~min}$ and then kept on ice for $2 \mathrm{~min}$. In half of the aliquots concentration of $\mathrm{Mg}^{2+}$ was increased to $7 \mathrm{mM}$ by adding of $1 / 10$ volume of $10 \times \mathrm{TAKM}_{7}$ buffer. We did not increase $\mathrm{Mg}^{2+}$ concentration in the other half of the aliquots, adding $1 / 10$ of volume of the same buffer but containing no $\mathrm{Mg}^{2+}$. After adding the buffer mRNAs were kept at room temperature for $30 \mathrm{~min}$ for renaturation and subsequent analysis by non-denaturating PAGE. 


\section{Mobility of mRNAs in a non-denaturing PAGE.}

10\% acrylamide gel (19:1) was prepared in TBE buffer (89 mM Tris-OH; 89 mM boric acid; 2 mM EDTA). 10 pmol of each fluorescent mRNA were loaded onto a gel after renaturation in the presence or absence of $7 \mathrm{mM}$ magnesium acetate. Gels were run at $80 \mathrm{~V}$ for $2.5 \mathrm{~h}$ at $4^{\circ} \mathrm{C}$ in TBE buffer and scanned using F7000 scanner with a fluorescence filter for Cy2 (GE Healthcare).

\section{Filter binding experiments.}

30S IC (0.3 $\mu \mathrm{M}$ 30S; 0.6 $\mu \mathrm{M}$ IF1; $0.6 \mu \mathrm{M}$ IF2; $0.6 \mu \mathrm{M}$ IF3; $0.9 \mu \mathrm{M}$ mRNA; $0.1 \mu \mathrm{M}\left[{ }^{3} \mathrm{H}\right.$ ]fMettRNA $^{\text {fMet }} ; 500 \mu \mathrm{M}$ GTP) was prepared in the $\mathrm{TAKM}_{7}$ buffer. For mRNA titration filter binding experiments varying concentrations of mRNA were used $(0.1 \mu \mathrm{M} ; 0.2 \mu \mathrm{M} ; 0.3$ $\mu \mathrm{M} ; 0.6 \mu \mathrm{M} ; 0.9 \mu \mathrm{M}$; same concentrations for the rest of the components as in reaction 2). Reaction mixtures were incubated at $37^{\circ} \mathrm{C}$ for $30 \mathrm{~min}$ and kept on ice for $10 \mathrm{~min}$.

The nitrocellulose filters were equilibrated ca. $30 \mathrm{~min}$ in the $\mathrm{TAKM}_{7}$ buffer before use. $50 \mu \mathrm{l}$ of the mixture containing initiation complexes were applied to the nitrocellulose filter, spreading the mixture evenly around the filter. The filter was washed from the unbound components with $3 \mathrm{ml}$ of the ice-cold TAKM 7 buffer. The filter was dissolved in $10 \mathrm{ml}$ of the scintillation cocktail Quickscint 361 (Zinsser Analytic) and radioactivity in the samples was measured by liquid scintillation counting. The amounts of $\left.{ }^{3} \mathrm{H}\right] \mathrm{fMet}-$ tRNA ${ }^{\text {fMet }}$ bound to the ribosome was calculated using the specific activity of $\left[{ }^{3} \mathrm{H}\right] \mathrm{fMet}$ $\operatorname{tRNA}^{\mathrm{fMet}}$. The efficiency of the initiation complex formation was calculated as the ratio of the amount of radioactive tRNA, bound to the filter, to the amount of the 30 s subunits added into the mixture.

\section{Dipeptide formation.}

Dipeptide was formed using prokaryotic in vitro translation system. The reactions were carried out in the TAKM 7 buffer. The reaction contained 30S IC (0.4 $\mu \mathrm{M}$ 30S; $0.8 \mu \mathrm{M}$ IF1; $0.8 \mu \mathrm{M}$ IF2; $0.8 \mu \mathrm{M}$ IF3; 1 mM GTP; $2 \mu \mathrm{M}$ mRNA; $1.2 \mu \mathrm{M}\left[{ }^{3} \mathrm{H}\right.$ ]fMet-tRNAfMet). Initiation complexes were incubated for $20 \mathrm{~min}$ at $37^{\circ} \mathrm{C}$ and kept on ice for $10 \mathrm{~min}$. After the incubation time, $50 \mathrm{~S}$ subunits $0.6 \mu \mathrm{M}$ were added to the initiation complex. Simultaneously ternary complexes were prepared (1.6 $\mu \mathrm{M}$ EF-Tu; $0.8 \mu \mathrm{M}\left[{ }^{14} \mathrm{C}\right]$ Phe-tRNA ${ }^{\text {Phe }} ; 1 \mathrm{mM}$ GTP; 3 mM phosphoenolpyruvate; $1 \%$ pyruvate kinase). 
Ternary complexes were incubated for $15 \mathrm{~min}$ at $37^{\circ} \mathrm{C}$. Equal volumes of the initiation and ternary complexes were mixed together and incubated for $5 \mathrm{~min}$ at room temperature. The reaction was stopped by addition of $1 / 10$ of the volume of $5 \mathrm{M} \mathrm{KOH}$. Before applying to the HPLC column the alkaline $\mathrm{pH}$ was neutralized with $100 \%$ acetic acid, and the total volume of the mixture adjusted to $200 \mu \mathrm{l}$ with $0.1 \%$ trifluoroacetic acid (TFA). The $\left[{ }^{3} \mathrm{H}\right] \mathrm{fMet}-\left[{ }^{14} \mathrm{C}\right] \mathrm{Phe}$ dipeptide was purified by the reverse-phase HPLC on the Chromolith RP-8e HPLC column, equilibrated in the $0.1 \%$ TFA. The sample was eluted from the column by a gradient of $0.1 \%$ TFA with $65 \%$ acetonitril. Radioactivity associated with HPLC fractions was measured both for ${ }^{3} \mathrm{H}$ and ${ }^{14} \mathrm{C}$ using double-label protocols for Packard 2500TR Liquid Scintillation Analyzer. The total amount of dipeptide was calculated based on the known specific activity of ${ }^{14} \mathrm{C}$-Phe $(1117 \mathrm{dpm} / \mathrm{pmol})$. The efficiency of dipeptide formation was shown in \% of the theoretical maximal possible dipeptide formation which is calculated based on the total amount of the ribosome in the reaction.

\section{Results.}

\section{Design of the mRNA set.}

All mRNAs used in this study were 30 nucleotides-long and labeled at the 3 '-end with Atto488 (ATTO-TEC GmbH, Siegen, Germany). mRNAs varied in the structure of the TIR, i.e. the length of the SD sequence (4 nucleotides, 9 nucleotides or none) and the presence or absence of the start codon (Table 2.1, Fig. 2.1). All mRNAs except m6a were designed such as not to have stable secondary structures. The secondary structure of mRNAs was predicted by Mfold software (Zuker, 2003) and the melting temperatures of the predicted folds were in the range between $25^{\circ} \mathrm{C}$ and $55^{\circ} \mathrm{C}$. One mRNA, m6a, was designed to form a hairpin structure so that its TIR was not accessible (Studer and Joseph, 2006). mRNA m6a was used as a negative reference control in binding experiments.

mRNAs were analyzed for the presence of actual secondary structure elements by nondenaturing PAGE (Fig 2.2). The influence of $\mathrm{Mg}^{2+}$ on the mRNA secondary structure was also checked. Renaturation of mRNAs was carried out in the presence of $7 \mathrm{mM} \mathrm{Mg}^{2+}$ or in the absence of magnesium ions. The folding of these mRNAs seems to be independent on the presence of $\mathrm{Mg}^{2+}$ but is greatly influenced by exchange of just two nucleotides in the primary sequence. 


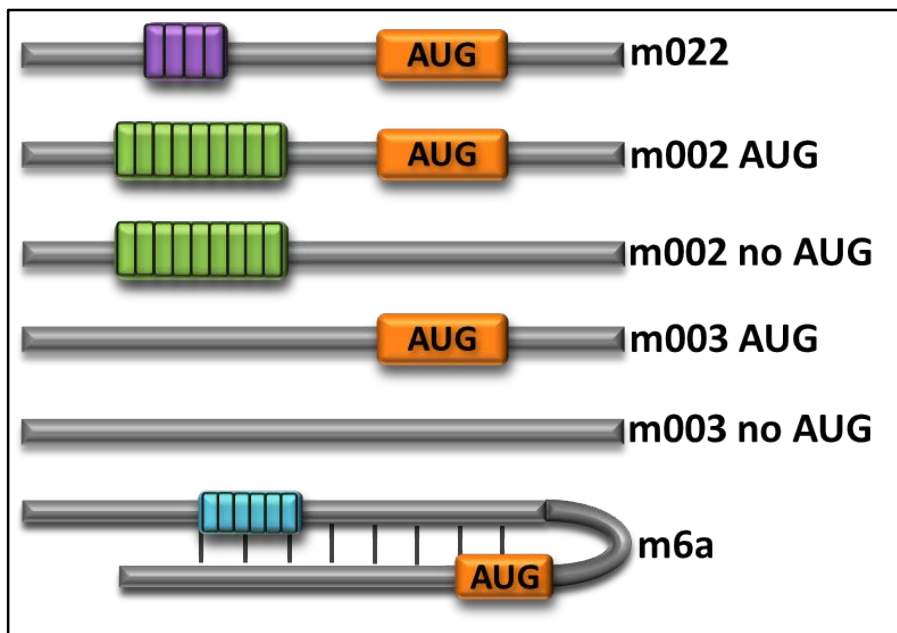

Figure 2.1 Schematic representation of mRNAs used in this work.

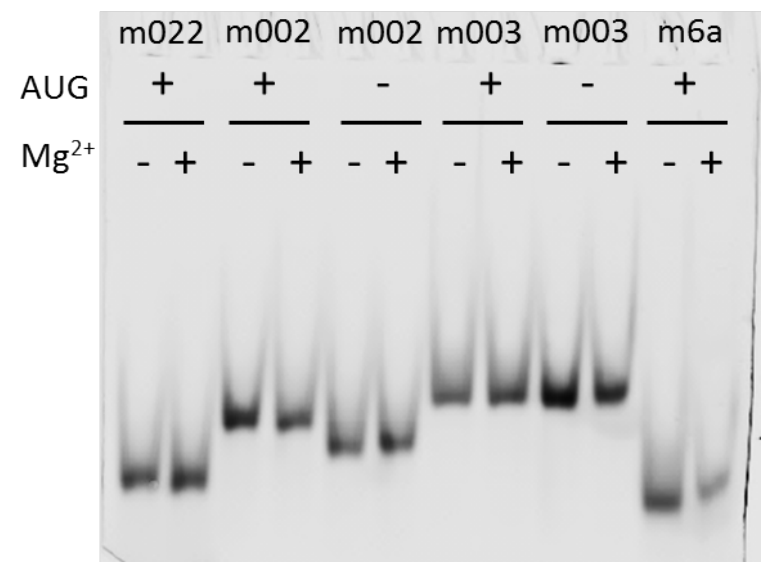

Figure 2.2 mRNA secondary structure analysis by nondenaturing PAGE.

Binding of mRNA to the 30S IC.

We checked whether fluorescent labeled synthetic mRNAs labeled with Atto-488 are capable of binding to the $30 \mathrm{SIC}$ in the presence of $\left[{ }^{3} \mathrm{H}\right] \mathrm{fMet}-\mathrm{tRNA}{ }^{\mathrm{Met}}$ by nitrocellulose filter binding (Figure 2.3). We took advantage of the fact that fMet-tRNA ${ }^{\text {fMet }}$ is stabilized on the $30 \mathrm{~S} \mathrm{IC} \mathrm{via} \mathrm{codon-anticodon} \mathrm{interaction,} \mathrm{allowing} \mathrm{us} \mathrm{to} \mathrm{indirectly} \mathrm{observe} \mathrm{the}$ presence of the mRNA in the complex to counting ${ }^{3} \mathrm{H}$ radioactivity of $\left[{ }^{3} \mathrm{H}\right]$ fMet-tRNA ${ }^{\text {Met }}$ retained in the complex with the $30 \mathrm{~S}$ subunits on the filter.

To test the effect of mRNA on the 30S IC formation, 30S IC was formed in excess of mRNA over other components (Fig. 2.3, A). We clearly see the pattern in $30 \mathrm{~S} \mathrm{IC}$ 
formation efficiency - the largest amount of the 30S IC (58 \%) is formed when the mRNA m002 AUG with the strongest SD (9 nt) is used, followed by m022 AUG with the efficiency of 30 S IC formation $41 \%$. When m002 with strong SD, but without AUG start codon was used for initiation, the efficiency of 30 S IC formation was $12 \%$, which is comparable to the control where no mRNA was used. This is explained by the fact that in the absence of the proper start codon, $\left[{ }^{3} \mathrm{H}\right] \mathrm{fMet}$-tRNA ${ }^{\mathrm{Met}}$ is not stabilized in the complex and dissociated easily. Curiously, when mRNA without SD sequence is used for the $30 \mathrm{~S}$ IC formation, the efficiency of complex formation is $29 \%$ in the presence of AUG start codon compared to $6 \%$ in the absence of AUG. Lacking the SD sequence, mRNA is not stabilized on the 30S subunit, hence low efficiency of 30S IC formation compared to when mRNAs with SD sequence is used. However, even in the absence of SD sequence, there is a difference in mRNA stabilization on the ribosome depending on the presence of the AUG start codon. Efficiency of the 30S IC formation using the hairpin m6a mRNA, the TIR of which is not accessible, was comparable to the 30S IC formation efficiency using the mRNAs without AUG start codon and/or without SD sequence. This finding supports the idea that TIR of the mRNA has to be exposed for the mRNA to be stabilized on the ribosome (Studer and Joseph, 2006). Alternatively, mRNA can have short singlestranded regions flanking the TIR which help its initial binding to the ribosome and provides time for TIR unwinding (De Smit and Van Duin, 2003).

We also performed 30S IC formation experiments using different amounts $(0.1 \mu \mathrm{M}-0.9$ $\mu \mathrm{M}$ ) of the mRNAs containing AUG codon to test whether TIR influences mRNA affinity to the ribosome (Fig. 2.3 B). Indeed, 30S IC formation with m002 AUG mRNA which has the strongest SD sequence reached saturation already at the lowest amount of mRNA used $(0.1 \mu \mathrm{M})$. The m022 AUG mRNA which has weaker SD than m002 AUG (4 nucleotides vs 9 nucleotides) has lower affinity to the 30S subunit. The m003 AUG mRNA which has no SD sequence has the lowest affinity to the 30S subunit of all the mRNAs tested. The 30S IC formation efficiency with mRNAs without AUG start codon and m6a mRNA was independent on the mRNA concentration and was indistinguishable from $30 \mathrm{~S}$ IC formation when no mRNA was used (data not shown). 

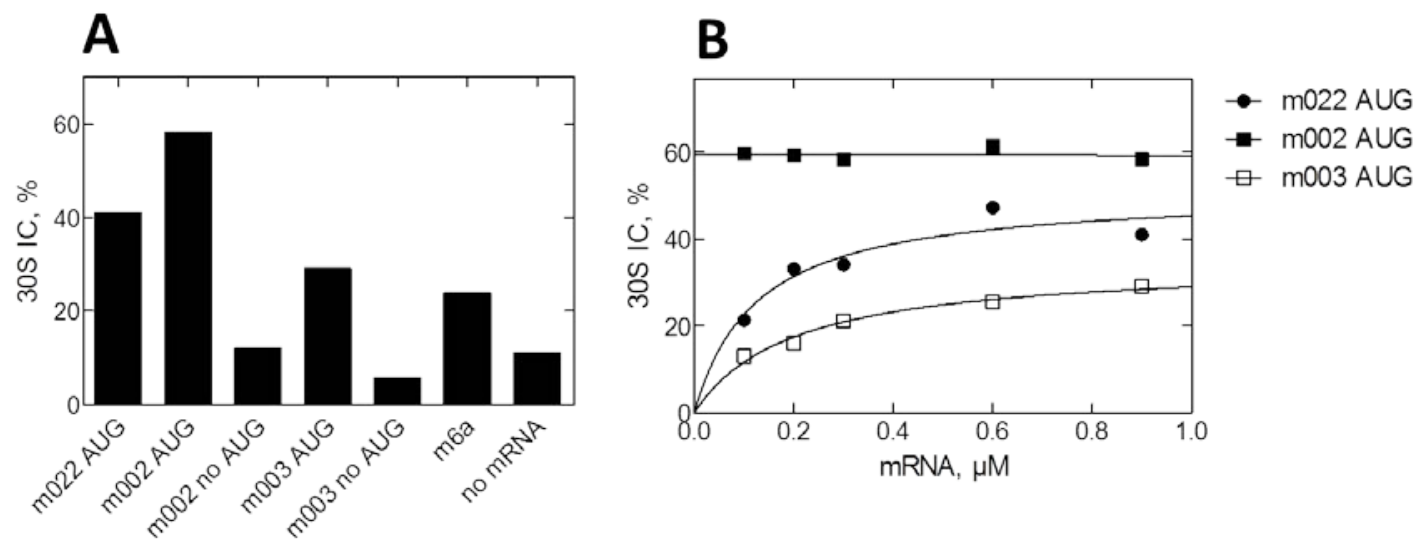

Figure 2.3 30S IC formation determined by filter binding. (A) Comparison of mRNAs differing in the presence of SD and AUG start codon. (B) Dependence from mRNA concentration.

\section{Dipeptide formation.}

To make sure that our mRNAs bind $30 \mathrm{~S}$ subunits and form functional initiation complexes we performed dipeptide formation experiments (Fig. 2.4). The preformed $70 S$ IC was mixed and incubated with the ternary complex containing EF-Tu and $\left[{ }^{14} \mathrm{C}\right]$ Phe-tRNA ${ }^{\text {Phe }}$ which resulted in the formation of $\left[{ }^{3} \mathrm{H}\right]$ Met- $\left[{ }^{14} \mathrm{C}\right]$ Phe dipeptide. The efficiency of dipeptide formation reflects the portion of properly formed initiation complexes which are able to progress to elongation. The results of dipeptide formation experiment showed that in the absence of AUG start codon there was no dipeptide formation. When AUG start codon was present in mRNA, the amount of dipeptide formed corresponded to the amount of 30S IC formed, reflecting efficiency of initiation on the particular mRNA. The amount of dipeptide formed on the m003 AUG mRNA lacking SD is close to that formed in case of m022 mRNA with weak SD which indicates that SD is not essential for translation initiation. Small amount of dipeptide formed on the complexes with m6a mRNA (9\%) possibly reflects the portion of mRNA that bound initiation complexes due to spontaneous unfolding and was stabilized on the ribosome. Even in the presence of mRNAs with strong SD and AUG start codon initiation efficiency did not reach $100 \%$. This can be possibly explained by partially inactive ternary complex or 50 s subunit.

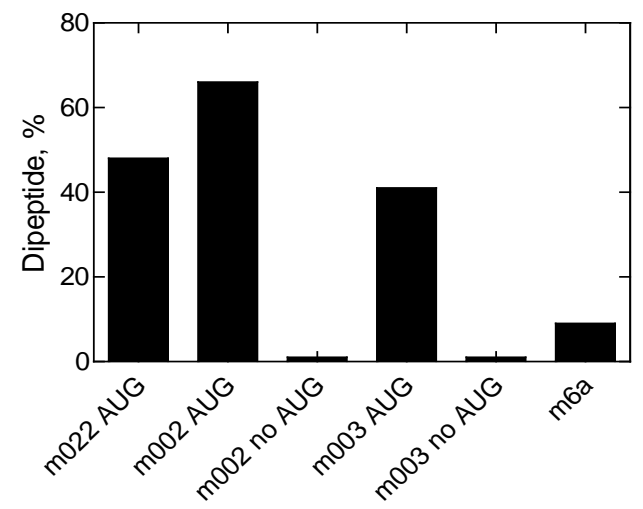

Figure 2.4 Dipeptide formation on 70S ICs formed with different mRNAs. 


\section{Binding of mRNA to the 305 subunit.}

We have observed that TIR structure of mRNA has impact on the $30 \mathrm{~S}$ and $70 \mathrm{~S} \mathrm{IC}$ formation. The differences in efficiencies of IC formation can be explained by different degree of mRNA stabilization in the complex depending on its TIR structure. To study the interaction of mRNA with the $30 \mathrm{~S}$ subunit directly, independent on fMet-tRNA ${ }^{\text {fMet }}$ or codon recognition, we used fluorescence-labeled mRNA and rapidly mixed them with the $30 \mathrm{~S}$ subunits in the stopped-flow apparatus. Binding of fluorescently labeled mRNAs to $30 \mathrm{~S}$ subunit led to the fluorescence change. To exclude the possibility of binding mRNA molecules to each other or fluorescence change upon dilution, the control experiment was performed where fluorescent mRNAs were mixed with buffer instead of the $30 \mathrm{~S}$ subunits (Fig. 2.5). Dilution of mRNA by buffer does not lead to fluorescence change.

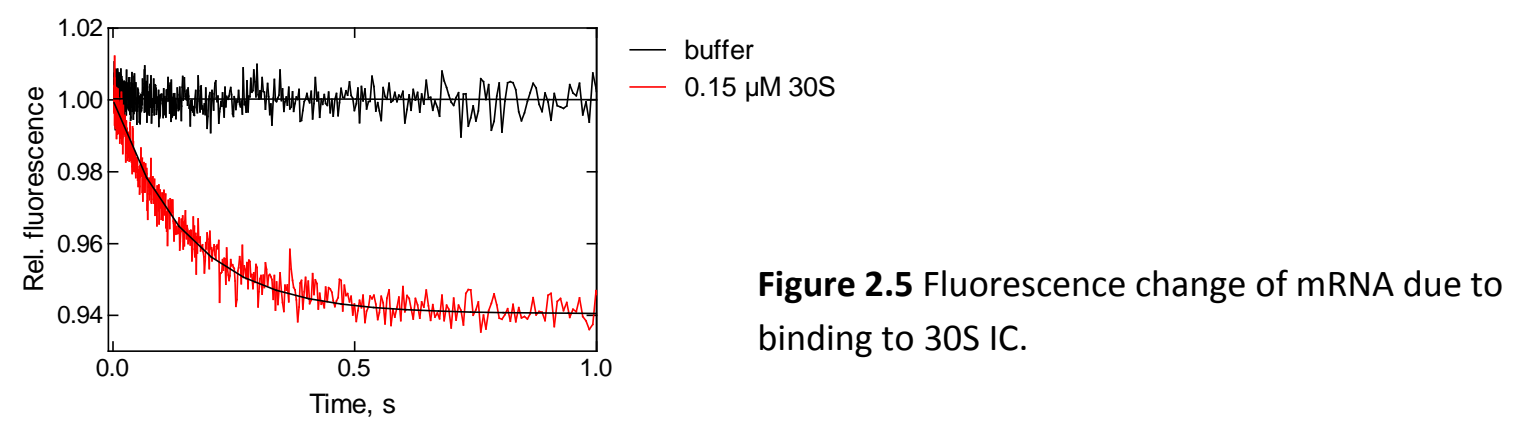

To test whether observed fluorescence change reflects interaction of single-stranded mRNA with the 30S subunit, we performed the control experiment where hairpin m6a mRNA was mixed with $30 \mathrm{~S}$ subunits (Fig. 2.6). There was no fluorescence change observed upon mixing of m6a with $30 \mathrm{~S}$ subunits supporting the notion that mRNA should not have stable secondary structure around TIR region to bind the ribosome (Studer and Joseph, 2006). Similarly, no fluorescence change of m6a mRNA was observed in the dissociation experiment where m6a was incubated with the $30 \mathrm{~S}$ and then mixed rapidly with the large excess of non-fluorescent m6a mRNA. This means that even after 30 min of incubation of the hairpin mRNA with the $30 \mathrm{~S}$ subunit, there is no binding, and hence, no dissociation of the m6a mRNA due to the stable secondary structure. For comparison, decrease in fluorescence was observed upon binding of m022 mRNA to the ribosome and increase in fluorescence upon its dissociation. 

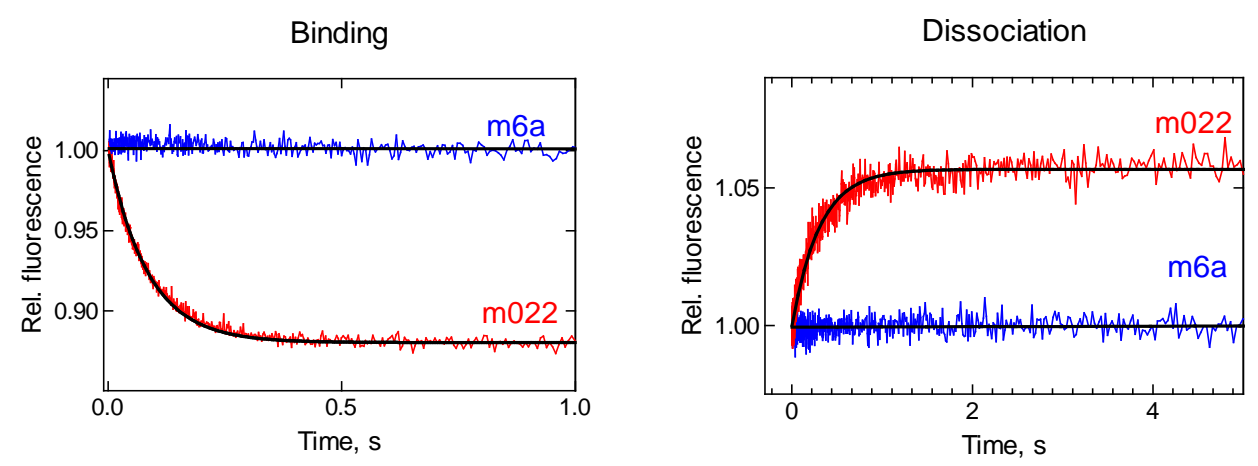

Figure 2.6 Binding of $\mathrm{m} 6 \mathrm{a}$ and $\mathrm{m} 022$ to the $30 \mathrm{~S}$ subunit.

\section{Kinetic parameters of mRNA binding the $30 \mathrm{~S}$ subunit.}

The binding experiments were carried out in a stopped-flow apparatus. Increasing concentrations of $30 \mathrm{~S}$ subunits were rapidly mixed with a constant concentration of fluorescent mRNAs. In most cases binding of mRNAs to the $30 \mathrm{~S}$ subunit resulted in decrease in fluorescence. The reactions were carried out at pseudo-first order conditions. The change in fluorescent signal was fitted by the single exponential function $Y=A 0+A 1 * \exp (-k 1 * t)$ or double exponential function $Y=A 0+A 1 * \exp (-k 1 * t)+A 2 * \exp (-k 2 * t)$ where $A 0$ corresponds to the final amplitude, $A 1, A 2$ to amplitudes of the corresponding phases and k1, k2 to the respective apparent rates. The dependence of the apparent rate constant on the concentration of $30 \mathrm{~S}$ subunits was linear, suggesting that a bimolecular association reaction was monitored. In such case the slope is equal to the association rate constant $\left(k_{o n}\right)$ and the $Y$-axis intercept indicates the dissociation rate constant $\left(k_{\text {off }}\right)$. In addition, chase experiments were performed to measure the $k_{\text {off }}$ value. The fluorescent mRNA-30S subunit complex was rapidly mixed with the access of unlabeled mRNA of the same type. The time courses of chase were fitted with single exponential equation; the apparent rate in this case corresponds to the $\mathrm{k}_{\text {off }}$.

\section{Binding of mRNAs to the $30 \mathrm{~S}$ subunit at $20^{\circ} \mathrm{C}$.}

For binding experiments concentration of mRNA was kept constant in all of the cases; each mRNA was titrated with 5 different concentrations of $30 \mathrm{~S}$ subunits. Binding of all mRNAs to the 30S subunit is shown in Figure 2.7. Rapid mixing of fluorescent mRNAs with the $30 \mathrm{~S}$ subunits resulted in decrease of fluorescent change in all but one case. The only exception was m002 no AUG mRNA, in which case binding of mRNA to the 30S led 
to increase in fluorescence at a fast rate followed by the slow decrease of fluorescence. The rate of the fast phase in this case is comparable to the association rates of other mRNAs. Therefore we assume that in case of m002 no AUG mRNA increase in fluorescence may also indicate binding. The possible explanation for the increase in fluorescence instead of decrease may be different folding of m002 no AUG mRNA compared to other mRNAs including m002 AUG mRNA. Upon binding of the mRNA to the 305 subunit, the environment of the fluorophore may change in a way that leads to the increase rather than decrease in fluorescence. Traces for m002 no AUG, m003 AUG and m003 no AUG mRNAs were fitted with double exponential function. In these cases the rates of the first (faster) phase were taken for further analysis as second (slower) phase is several orders of magnitude slower than possible subsequent initiation events. The apparent rate constants of mRNA binding to $30 \mathrm{~S}$ were derived from exponential fitting of the traces and are summarized in Fig 2.8 and Table 2.3. The apparent rate constants for the tested mRNAs were in the range of $15-60 \mu \mathrm{M}^{-1} \mathrm{~s}^{-1}$. There was no apparent correlation between the association rate constants and mRNA TIR sequence.

Dissociation constant can be determined by two different approaches. It can be be either derived from the linear dependence of the $k_{\text {app }}$ values on the 305 concentration or measured directly by chase experiments. Theoretically these two values should be the same; however the experiments showed up to 10 -fold different values depending on how the dissociation rate constants were measured, with those measured by chase experiments being always lower (Table 2.2). We suggest the following explanation to this observation. mRNA first binds the ribosome via a standby site, from which it can dissociate rapidly. This value is reflected in the intercepts of the $k_{\text {app }}$ dependencies on the $30 \mathrm{~S}$ subunit concentration (denoted as $k_{-1}$ here). Subsequently, mRNA accommodates in the initiation site which increases the stability of its binding to the ribosome and results in slower dissh8ociation when the chase from preformed complexes is monitored (Fig. 2.10). 


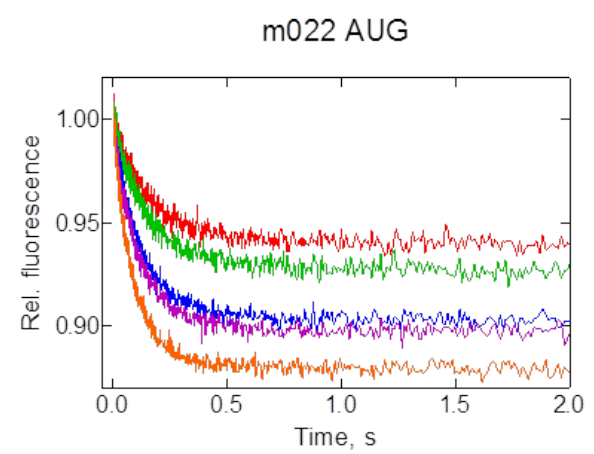

$-0.15 \mu \mathrm{M} 30 \mathrm{~S}$

- $0.2 \mu \mathrm{M} 30 \mathrm{~S}$

$-0.3 \mu \mathrm{M} 30 \mathrm{~S}$

- $0.4 \mu \mathrm{M} 30 \mathrm{~S}$

- $0.5 \mu \mathrm{M} 30 \mathrm{~S}$

m002 AUG

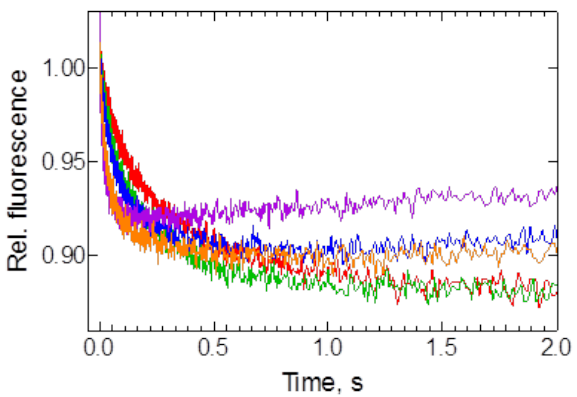

m002 no AUG

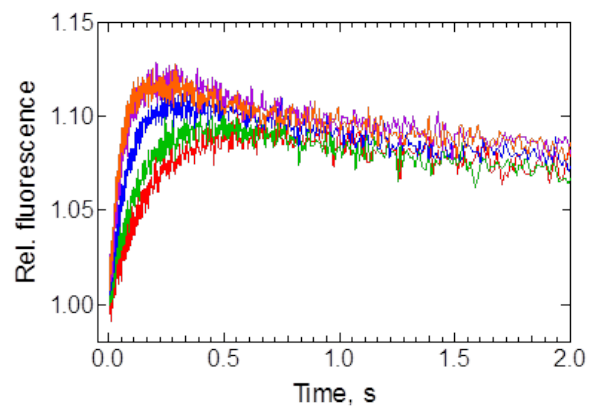

m003 AUG
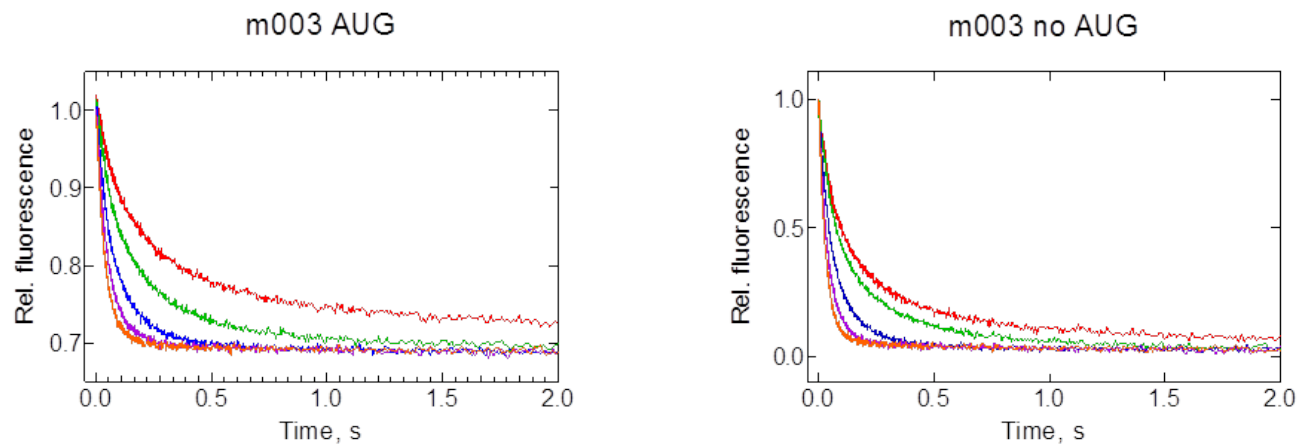

Figure 2.7 Binding of mRNAs to the $30 \mathrm{~S}$ subunit. See explanations in the text.

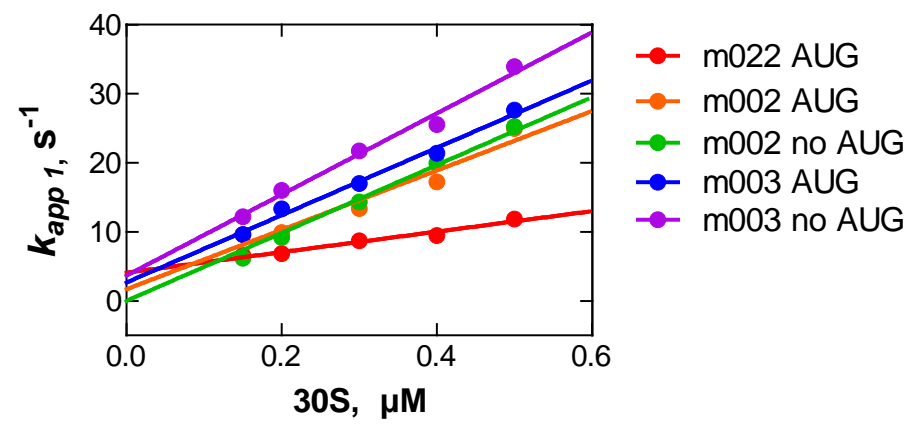

Figure 2.8 Concentration dependence of $k_{a p p}$ values for mRNA binding to 30S subunits measured for different mRNAs. 

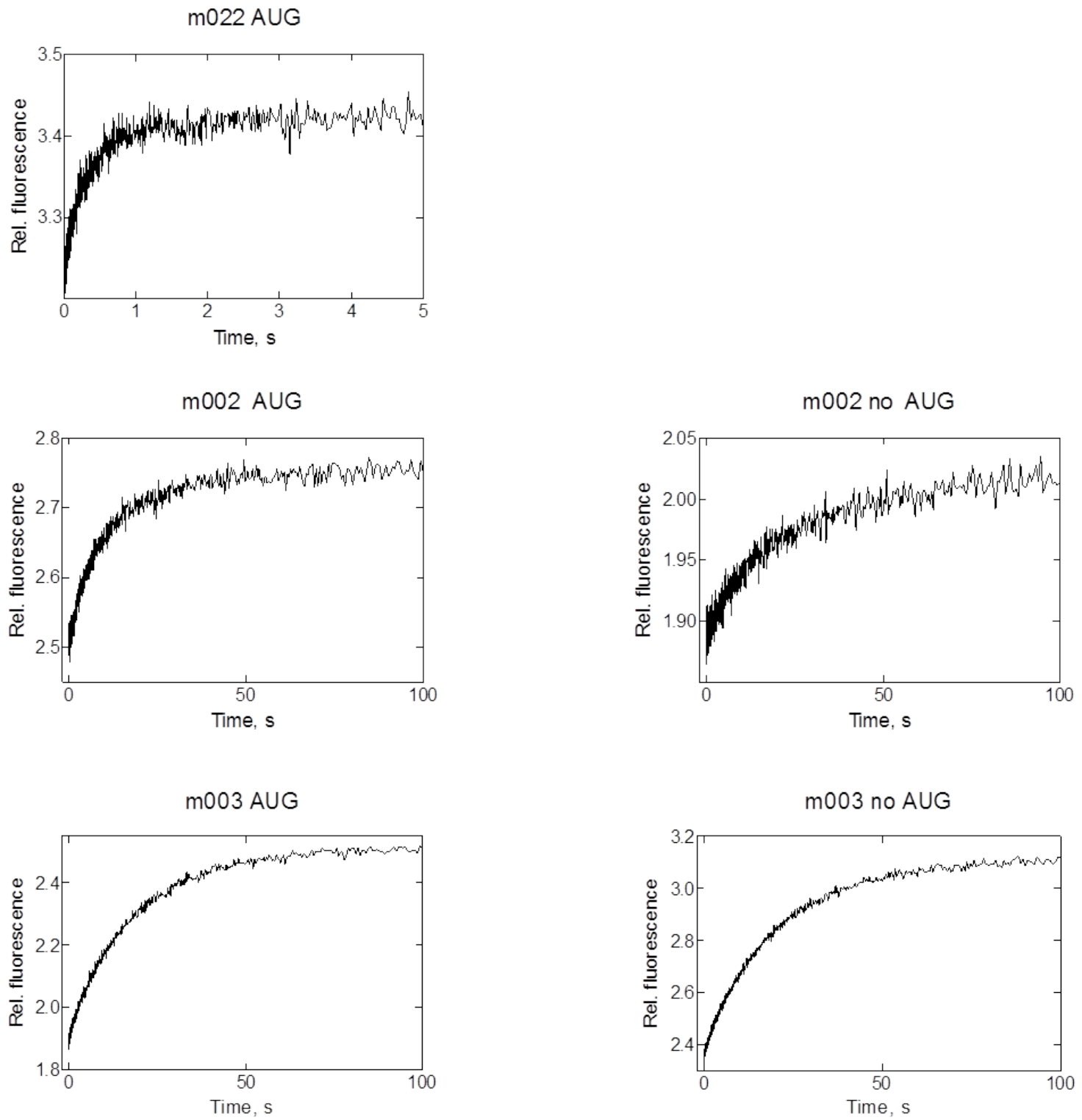

Figure 2.9 mRNA dissociation from the $30 \mathrm{~S}$ subunit.

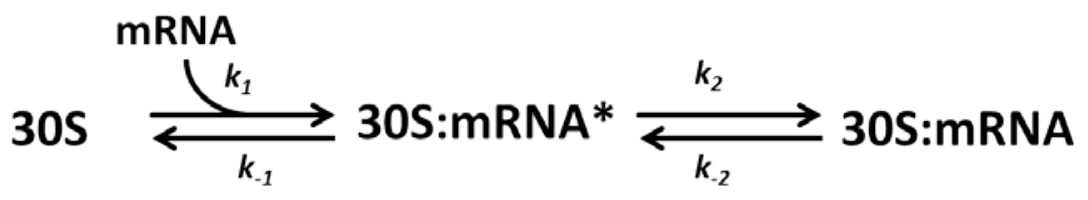

Figure 2.10 Interaction of mRNA with the $30 \mathrm{~S}$ subunit. Two steps of interaction are schematically represented: (1) the binding of the mRNA to the standby site and (2) mRNA accommodation to the $P$ site in the ribosome. 


\section{Binding of mRNAs to the $30 \mathrm{~S}$ subunits at $37^{\circ} \mathrm{C}$.}

In the series of experiments performed at $20^{\circ} \mathrm{C}$, the association rate constants were approximately the same in all cases. We did not observe any dependence of mRNA primary binding to the $30 \mathrm{~S}$ subunit on the TIR structure. This could be due to some secondary structures that mRNAs may form at $20^{\circ} \mathrm{C}$ and render the TIR inaccessible for the interaction with the $30 \mathrm{~S}$ subunit. Increase of temperature would destabilize possible secondary mRNA structures and TIR would be exposed for the interaction with the ribosome, which prompted us to repeat the experiments at $37^{\circ} \mathrm{C}$.

Fluorescent changes upon interaction of mRNAs with the $30 \mathrm{~S}$ subunits at $37^{\circ} \mathrm{C}$ are shown in Fig. 2.11. Binding of all mRNAs except m002 no AUG to the 30 s subunit led to decrease in fluorescence. Binding of m002 no AUG led to increase of fluorescence which is consistent with data obtained at $20^{\circ} \mathrm{C}$, suggesting that increase of temperature to $37^{\circ} \mathrm{C}$ did not alter secondary structure of m002 no AUG if this was the reason for different behavior of this mRNA in comparison to the other mRNAs from this set. The dependencies of the $k_{\text {app }}$ values on the 30S subunit concentration are shown in Fig. 2.12. The values of association rate constants calculated from these dependencies are summarized in Table 2.4. As expected, all the apparent rates are faster at $37^{\circ} \mathrm{C}$. However, we still did not find any correlation between the association rate constants and TIR structure.

The dissociation rate constant was measured at $37^{\circ} \mathrm{C}$ by chasing the fluorescencelabeled mRNA from the preformed complexes with the $30 \mathrm{~S}$ subunit by non-labeled mRNA of the same type (Fig. 2.13). Exactly as with binding experiments, the dissociation rates at $37^{\circ} \mathrm{C}$ were faster than at $20^{\circ} \mathrm{C}$ but there was no correlation with the primary TIR structure (Table 2.4).

\section{Binding of mRNAs to the 30S PIC and 30S IC.}

mRNA is thought to bind $30 \mathrm{~S}$ initiation complex independent on the presence of initiation factors or fMet-tRNA ${ }^{\text {fMet }}$ (Calogero et al., 1988; Milón et al., 2012b; Studer and Joseph, 2006). However if IFs and/or fMet-tRNA ${ }^{\text {fMet }}$ are already bound to the $30 \mathrm{~S}$ subunit, the conformation of the $30 \mathrm{~S}$ subunit may be altered possibly creating more favorable environment for the mRNA to bind. We aimed to test whether the TIR 
structure influences the primary binding of mRNA in the presence of IFs and fMettRNA $^{\text {fMet }}$.

m022 AUG

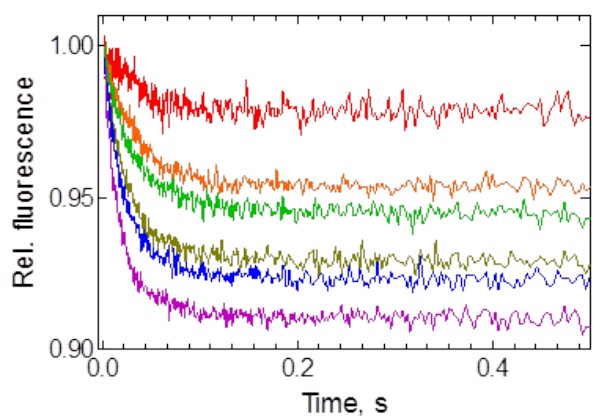

m002 AUG

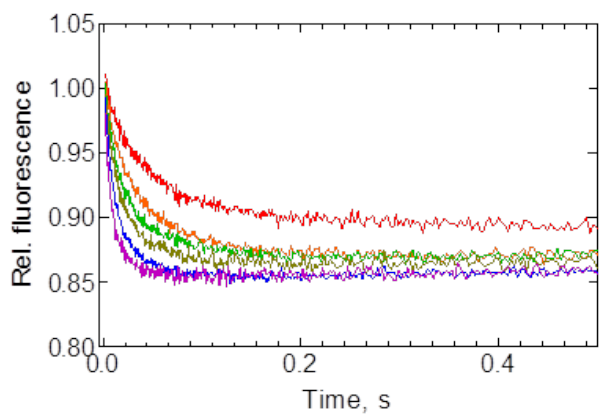

m003 AUG

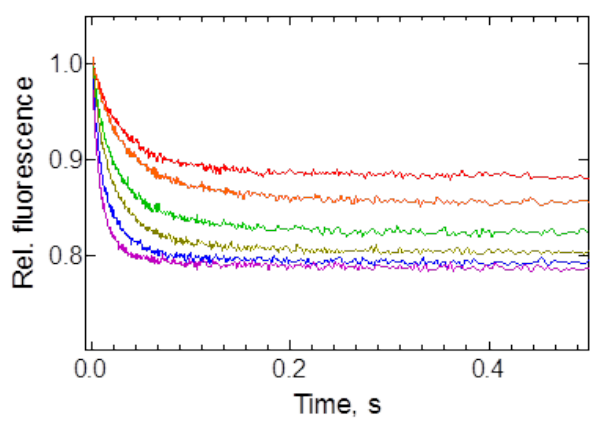

m002 no AUG

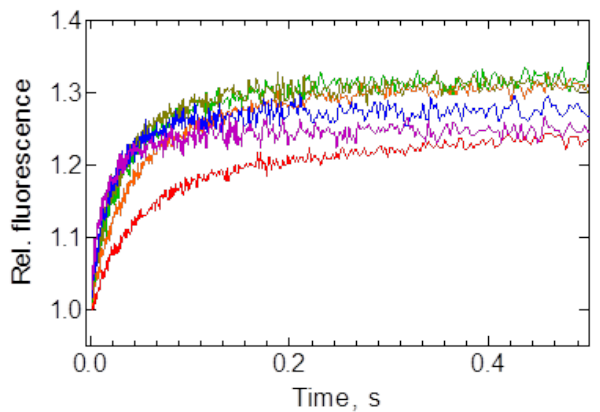

Figure 2.11 Time courses of mRNA binding to the $30 \mathrm{~S}$ subunit at $37^{\circ} \mathrm{C}$.

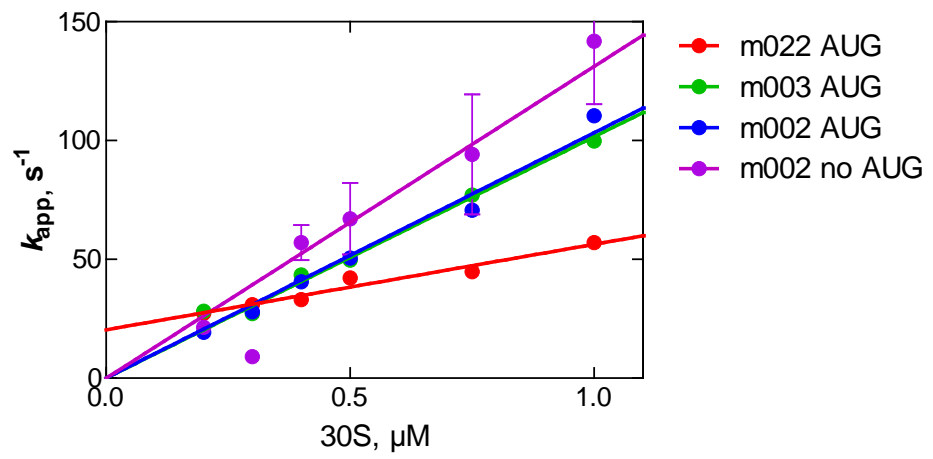

Figure 2.12 Dependence of the $k_{\text {app }}$ values on $30 \mathrm{~S}$ concentration at $37^{\circ} \mathrm{C}$. 
30S PIC and 30S IC were prepared and rapidly mixed with fluorescently labeled mRNAs in a stopped-flow apparatus. Unlike in the titration experiments of mRNA binding to the vacant 30S, we tested only single concentration of $30 \mathrm{~S} \mathrm{PIC/IC} \mathrm{for} \mathrm{binding} \mathrm{to} \mathrm{mRNAs} \mathrm{(Fig.}$ 2.14). Experiments of dissociation of mRNA from the 30S PIC/IC were performed in the same way as for mRNA dissociation from the vacant $30 \mathrm{~S}$ (Fig. 2.14).
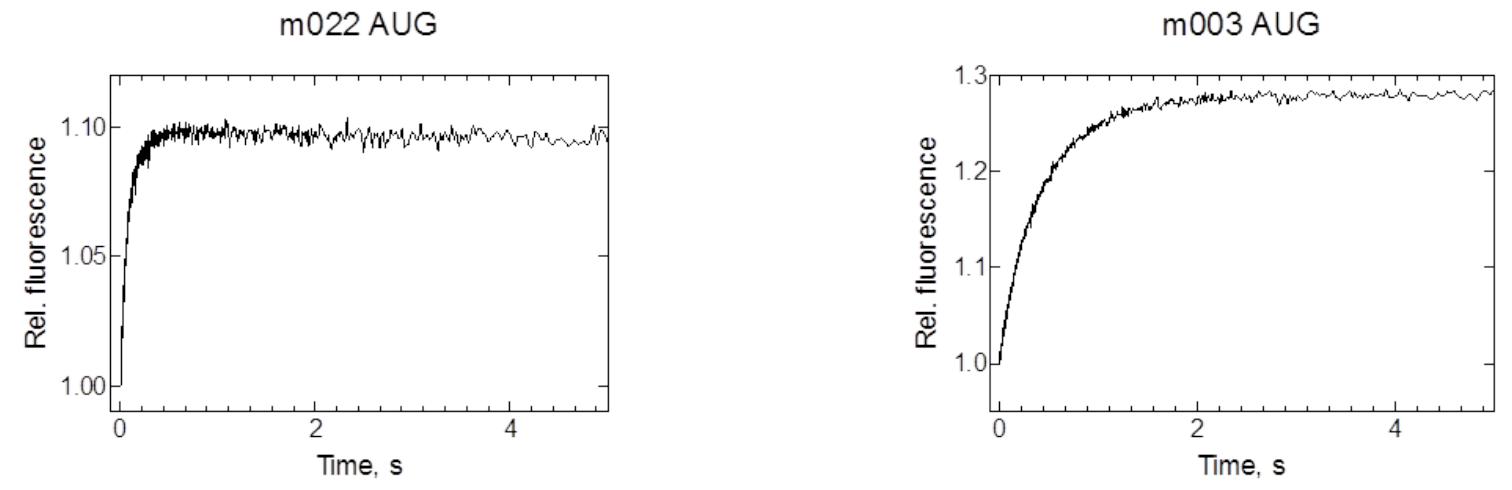

Figure 2.13 Dissociation of mRNAs from the $30 \mathrm{~S}$ at $37^{\circ} \mathrm{C}$.

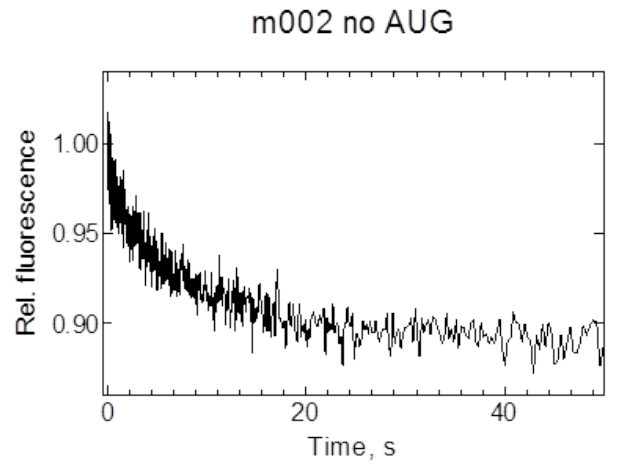

Measured $k_{a p p}$ values are summarized in Fig. 2.15. The $k_{a p p}$ values were independent of whether IFs and fMet-tRNA ${ }^{\text {fMet }}$ were present in the 30 S complex. Again, there was no obvious correlation between the mRNA binding to the 30S/30S PIC/30S IC and its TIR structure. The observed differences in the association rates can be rather explained by possible variations in the secondary structures of these mRNAs.

Dissociation experiments showed that mRNA was stabilized on the 30S IC when fMet$\mathrm{tRNA}^{\mathrm{fMet}}$ was present. This stabilization occurs via codon-anticodon interaction. The dissociation of m022 AUG and m002 AUG from 30S IC is not detectable. Both mRNAs have an AUG start codon and a SD sequence, although of different strength. mRNA m003 AUG, which has an AUG start codon, dissociates independently of whether fMettRNA $^{\text {fMet }}$ is present in the complex. Due to the lack of the SD sequence, m003 AUG may be incorrectly positioned on the ribosome, so that the AUG will not be in the P site of the ribosome. However, a portion of the m003 AUG mRNA is positioned correctly which 
is proven by its ability to stimulate 30S IC formation (Fig. 2.3) and formation of the functional 70S IC (Fig. 2.4). The efficiency of the 30S IC formation with m003 AUG was 29 $\%$, which is significant, compared to $41 \%$ of the 30 S IC formation with the m022 AUG (Fig. 2.3). Also, dipeptide formation efficiency with m003 AUG was $41 \%$, compared to 48 $\%$ when m022 AUG was used (Fig. 2.4). These facts suggest that a portion of m003 AUG is able to establish the correct reading frame with the AUG in the $P$ site of the ribosome. This portion of mRNA is stabilized on the ribosome by the fMet-tRNA ${ }^{\mathrm{fMet}}$ the same way, as SD-containing mRNAs are, and therefore its dissociation is undetectable. The other portion of m003 AUG mRNA does not have its AUG in the $P$ site of the ribosome and therefore dissociates even in the presence of the fMet-tRNA ${ }^{\text {fMet }}$. 

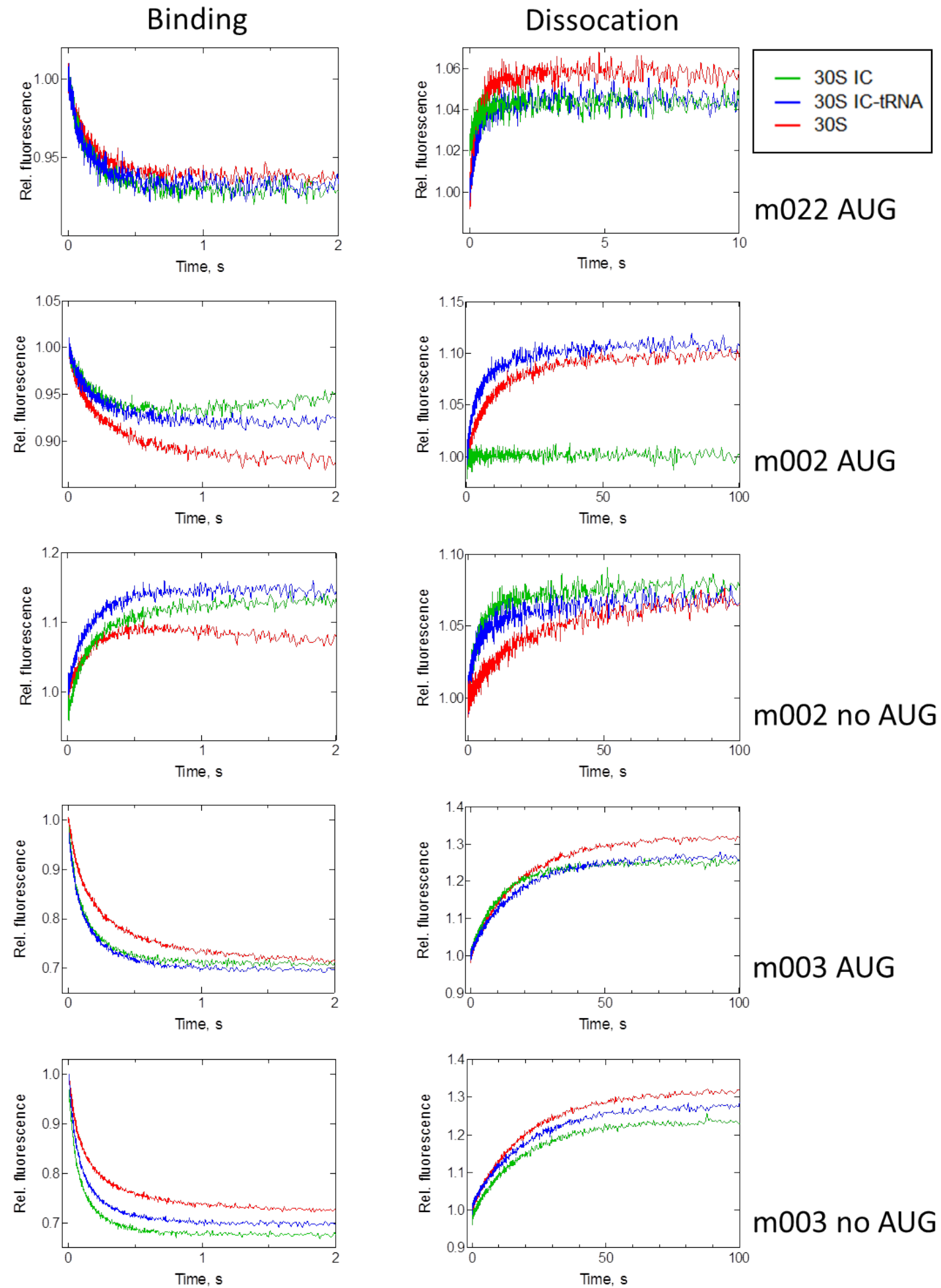

Figure 2.14 Binding and dissociation of mRNAs from 30S/30S PIC/30S IC. 

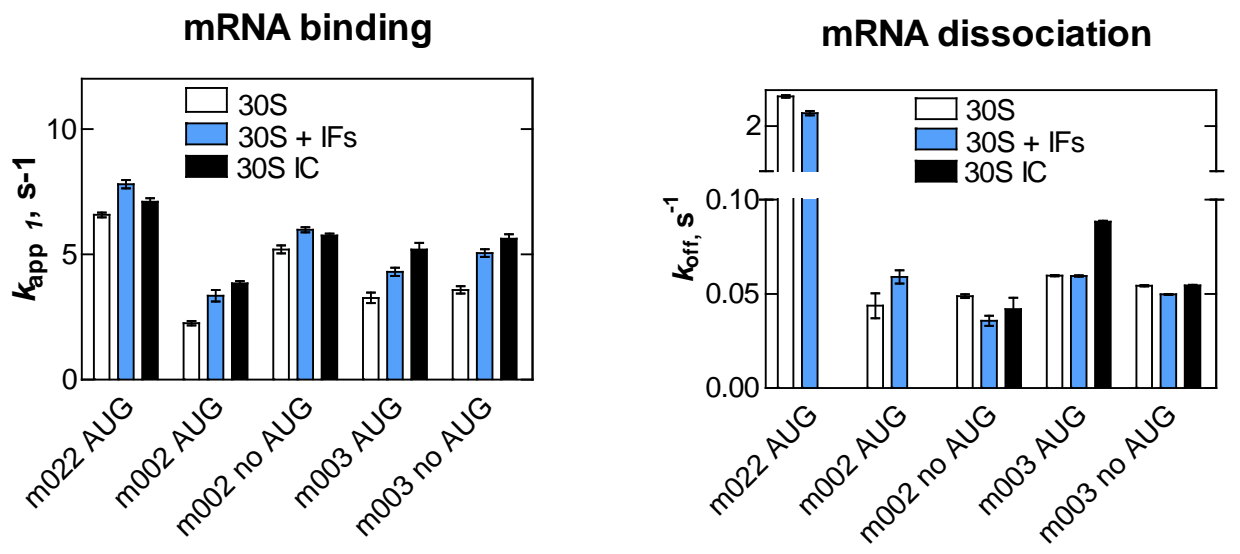

Figure 2.15 Association and dissociation rate constants of mRNAs binding to $30 \mathrm{~S}$ complexes.

Table 2.2 Association and dissociation rate constants of mRNAs binding to/dissociation from the $30 \mathrm{~S}$ complexes at $20^{\circ} \mathrm{C}$.

\begin{tabular}{|l|l|l|l|l|l|l|}
\hline $20^{\circ} \mathrm{C}$ & \multicolumn{3}{l}{$30 \mathrm{~S}$} & \multicolumn{2}{l|}{$30 \mathrm{~S}$ PIC } & \multicolumn{2}{l|}{ OS IC } \\
\hline & $\begin{array}{l}k_{\text {on }} \\
\mu \mathrm{M}^{-1} \mathrm{~s}^{-1}\end{array}$ & $\begin{array}{l}k_{\text {off }} \\
\mathrm{s}^{-1}\end{array}$ & $\begin{array}{l}k_{\text {on }} \\
\mu \mathrm{M}^{-1} \mathrm{~s}^{-1}\end{array}$ & $\begin{array}{l}k_{\text {off }} \\
\mathrm{s}^{-1}\end{array}$ & $\begin{array}{l}k_{\text {on }} \\
\mu \mathrm{M}^{-1} \mathrm{~s}^{-1}\end{array}$ & $k_{\text {off }}$ \\
\hline m022 AUG & $15 \pm 2$ & $3 \pm 0.1$ & $36 \pm 1$ & $2.5 \pm 0.1$ & $48 \pm 1$ & n.d. \\
\hline m002 AUG & $43 \pm 6$ & $0.05 \pm 0.005$ & $40 \pm 1$ & $0.06 \pm 0.005$ & $38 \pm 1$ & n.d. \\
\hline m002 no AUG & $54 \pm 1$ & $0.05 \pm 0.005$ & $46 \pm 1$ & $0.04 \pm 0.005$ & $45 \pm 1$ & $0.04 \pm 0.01$ \\
\hline m003 AUG & $49 \pm 3$ & $0.06 \pm 0.005$ & $84 \pm 1$ & $0.06 \pm 0.005$ & $95 \pm 1$ & $0.09 \pm 0.001$ \\
\hline m003 no AUG & $59 \pm 4$ & $0.05 \pm 0.005$ & $118 \pm 2$ & $0.05 \pm 0.005$ & $112 \pm 2$ & $0.05 \pm 0.001$ \\
\hline
\end{tabular}


Table 2.3 Association and dissociation rate constants of mRNAs binding to $30 \mathrm{~S}$ complexes at $37^{\circ} \mathrm{C}$.

\begin{tabular}{|l|l|l|}
\hline $37^{\circ} \mathrm{C}$ & \multicolumn{2}{|l|}{$30 \mathrm{~S}$} \\
\hline & $k_{\text {on }}$ & $k_{\text {off }}$ \\
& $\mu \mathrm{M}^{-1} \mathrm{~s}^{-1}$ & $\mathrm{~s}^{-1}$ \\
\hline $\mathrm{m} 022$ AUG & $36 \pm 4$ & $13 \pm 0.1$ \\
\hline m002 AUG & $110 \pm 7$ & $1.2 \pm 0.05$ \\
\hline m002 no AUG & $94 \pm 11$ & $1 \pm 0.1$ \\
\hline m003 AUG & $95 \pm 6$ & $2.8 \pm 0.02$ \\
\hline
\end{tabular}

\section{Discussion.}

The efficiency of recruitment of a particular mRNA to the 30S PIC depends on the mRNA abundance in the sell as well as its TIR structure (Kudla et al., 2009; McCarthy and Gualerzi, 1990). Such parameters as the stability of the TIR secondary structure, the strength of SD-aSD interaction and the start codon recognition determine the efficiency of the $30 \mathrm{~S}$ PIC formation. In the cell, there is a number of checkpoints for mRNA selection (Fig 2.16) (Milón et al., 2012b). The first checkpoint occurs whens mRNAs with various TIR structure compete for $30 \mathrm{~S}$ binding (Calogero et al., 1988). At this time point any mRNA having short single-stranded regions shows fast association with the 30S subunit (Studer and Joseph, 2006). The mRNA binds to the standby site at the platform of the 30 S subunit (Marzi et al., 2007; Yusupova et al., 2006). Subsequently the secondary structure of TIR unfolds and mRNA is adjusted in the mRNA-binding channel. At this step there is the second kinetic checkpoint. Whether mRNA remains bound to the ribosome depends on the ratio between the rate of mRNA dissociation form the $30 \mathrm{~S}$ subunit and the rate of mRNA unfolding (Milón et al., 2012b). An mRNA with stronger secondary structure unfolds slower and is more likely to be released form the ribosome prior to being stabilized (Studer and Joseph, 2006). Upon SD-aSD interaction and 
positioning of the start codon in the $\mathrm{P}$ site of the ribosome, the start codon recognition by the fMet-tRNA ${ }^{\text {fMet }}$ takes place. Here the third mRNA checkpoint occurs, as the codon recognition acts as an affinity switch that stabilizes the $m R N A$ and $f M e t-t R N A^{\text {fMet }}$ in the complex (Milón et al., 2012b). The mRNAs with the canonical AUG start codon are favored, while the presence of a non-canonical initiation codon destabilizes the 30S IC formation (Milon et al., 2008). The last checkpoint occurs at the transition of the $30 \mathrm{~S} \mathrm{IC}$ to the 70S IC where the structural elements of TIR and the correct start codon recognition are monitored (Antoun et al., 2006a; Milon et al., 2008). The mRNA with the optimal combination of elements promotes the conformation of the $30 \mathrm{~S} \mathrm{IC} \mathrm{that}$ facilitates the 50 S subunit joining (Grigoriadou et al., 2007a; Julián et al., 2011; Milon et al., 2008; Simonetti et al., 2009). The presence of the mRNA with unfavorable TIR slows down the 70S IC formation and leads to lower mRNA expression (Milon et al., 2008). Alternatively, if there is no codon-anticodon recognition, 50S may dissociate from the initiation complex. The formation of the 30S PIC and its transition to the 30S IC and 70S IC provides four kinetic checkpoints for the mRNA selection. At each checkpoint mRNA can either dissociate from or be stabilized in the initiation complex depending on its structure and thus, the fate of the mRNA is decided (Milón et al., 2012b).

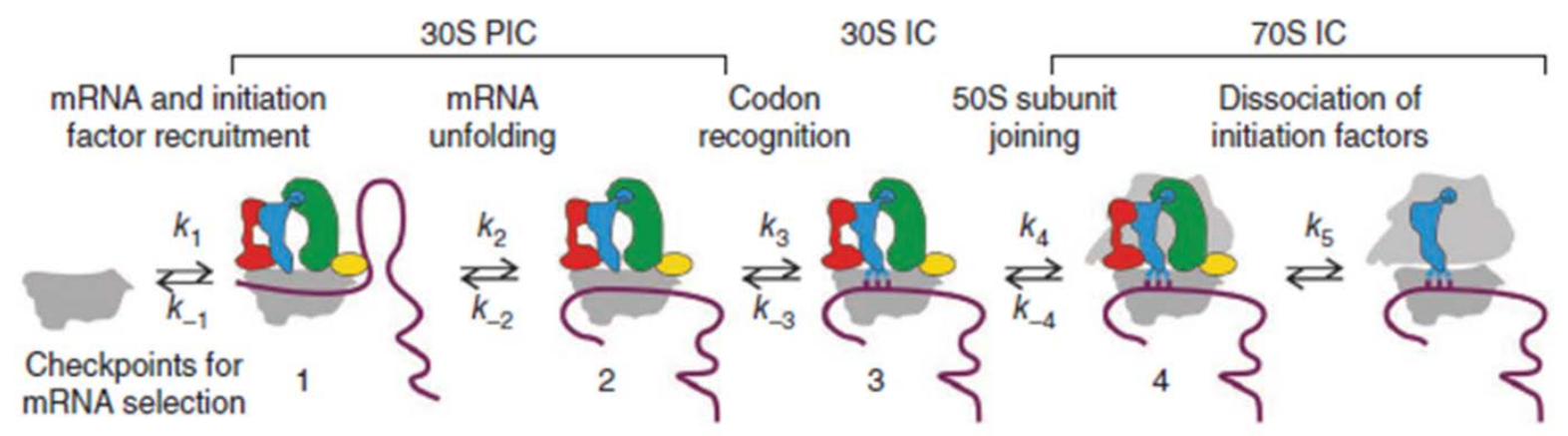

Figure 2.16 (from Milon et al., 2012) Checkpoints of mRNA selection. The kinetic partitioning checkpoints operate at the stages of mRNA recruitment (checkpoint 1), mRNA unfolding and transition from standby to initiation sites (checkpoint 2), start codon recognition and transition from the 30 S PIC to the 30 S IC complex (checkpoint 3) and upon 50S subunit joining and transition from the 30 S IC to the 70 SIC (checkpoint 4).

In the scope of the current work we were interested in the kinetics of the primary mRNA binding to the $30 \mathrm{~S}$ subunit. The influence of the mRNA TIR structure, initiation factors and $f M e t-t R N A^{f M e t}$ on the mRNA binding was investigated. We designed a set of fluorescently labeled mRNAs which differed in the TIR structure to study whether the SD 
sequence and the presence of the AUG start codon influence the mRNA binding kinetics or its stability of binding to the 30S IC. mRNAs had either weak or strong Shine-Dalgarno sequence, or it was absent at all. mRNAs were designed not to have stable secondary structures such that TIR was available for the interaction with the ribosome. One of the mRNAs (m6a) which formed a closed hairpin structure was used as a negative control for binding experiments. Different secondary structures of mRNAs were predicted by Mfold and confirmed by mRNA mobility in the nondenaturing PAGE. The mRNAs were tested for the stimulation of the 30S IC formation as well as for the functional 70S IC formation. The mRNAs which contained AUG codon were found to stabilize fMet-tRNA ${ }^{\text {fMet }}$ in the 30S IC and stimulate dipeptide formation independently on the SD presence, although to a different extent depending on the SD presence and strength.

We checked the set of mRNAs for binding to and dissociation from vacant 30 S ribosomal subunits, 30S PIC (lacking fMet-tRNA ${ }^{\text {fMet }}$ ) and $30 \mathrm{~S} \mathrm{IC.} \mathrm{In} \mathrm{most} \mathrm{cases} \mathrm{a} \mathrm{change} \mathrm{in}$ fluorescence upon mRNA binding or dissociation from the ribosome was observed. Based on our experiments we were able to calculate association and dissociation rate constants for all mRNAs studied. The rates of binding of all mRNAs to vacant 30 S subunit or 30S PIC/IC did not show any significant differences. The possible explanation for this is that 30 subunit has initial strong affinity to any kind of single stranded RNA. This also suggests that primary binding of the mRNA to the ribosome does not depend on the presence of the SD sequence and the start codon. The rates of dissociation from the vacant 30S subunit were approximately the same for all of the mRNAs tested (except m022 AUG). We also conducted binding and dissociation experiments with vacant $30 \mathrm{~S}$ subunits at $37^{\circ} \mathrm{C}$ to overcome the possible secondary structures in mRNA. No dependence of mRNA binding and dissociation on the TIR structure was found. When mRNA dissociation from the 30S IC was measured, m022 AUG and m002 AUG were greatly stabilized on the complex and did not dissociate. Dissociation rates of all other mRNAs from the $30 \mathrm{SIC}$ were similar.

If fMet-tRNA ${ }^{\text {fMet }}$ was omitted from the complex, m022 AUG and m002 AUG dissociated from the 30S PIC the same way as all other mRNAs. This suggests that codon-anticodon interaction is important for mRNA stabilization on the 30S IC but not SD-aSD interaction. This is supported by the finding that m002 lacking AUG codon dissociated from the complex containing fMet-tRNA ${ }^{\mathrm{fMet}}$. The mRNA construct m022 AUG exhibited much faster dissociation from the vacant $30 \mathrm{~S}$ and $30 \mathrm{~S}$ PIC than the rest of the mRNAs. This difference in the dissociation rates can possibly be explained by the differences in the 
secondary structures of mRNAs rather than TIR structure. Moreover, upon addition of fMet-tRNA ${ }^{\text {fMet }}$, m022 AUG was stabilized in the complex similarly to the m002 AUG.

Both m003 mRNAs which lacks SD show the same association rates regardless the presence of the start codon. The mRNA m003 AUG is not stabilized in the presence of fMet-tRNA ${ }^{\text {fMet }}$ most likely due to the incorrect positioning on the ribosome (start codon is not in the $\mathrm{P}$ site). However there is evidence that proper initiation is possible on the m003 AUG mRNA even in the absence of SD-aSD interaction (Calogero et al. 1988 and this work). In such a case the explanation is that there is a portion of this mRNA that has AUG codon in the P site and a portion that is incorrectly positioned.

Our work shows that the primary arrival of mRNA to the ribosome is independent from its TIR structure. TIR was shown to play an important role on the later steps of mRNA stabilization on the 30S IC. We also found that the presence of initiation factors and/or $\mathrm{fMet} \mathrm{tRNA}{ }^{\mathrm{fMet}}$ in the $30 \mathrm{~S}$ IC does not influence the primary docking of the mRNA. Our data support previous observations that initial mRNA docking on the ribosome strongly correlates with mRNA abundance in the cell (Passalacqua et al., 2009). We conclude that during the assembly of 30S PIC mRNA binds to the complex in parallel but independent on the other components (Milón et al., 2012b). It was shown earlier that binding of IF1, IF2, IF3 and FMet-tRNA ${ }^{\text {fMet }}$ trigger global conformational changes in the 30 s subunit (Allen et al., 2005; McCutcheon et al., 1999; Pioletti et al., 2001; Wakao et al., 1991). Although the initiation factors appear to have no role in direct stabilization of mRNA in the IC, the conformation changes induced by initiation factors binding stabilize fMettRNA $^{\text {fMet }}$ in the complex. The initiator tRNA in its turn stabilizes mRNA via codonanticodon interaction. Conformational changes of the $30 \mathrm{~S}$ subunit also help SD-aSD interaction to be formed (Gualerzi and Pon, 1990; de Smit and van Duin, 1994; La Teana et al., 1996). Whether mRNA remains bound to the ribosome during translation initiation depends on the ratio between mRNA dissociation from the 3OS PIC and MRNA stabilization. We also showed that there is an affinity switch upon start codon recognition. mRNAs with AUG codon in the P site are stabilized in the 30S PIC if fMettRNA ${ }^{\text {fMet }}$ is present. Thus, on the basis of kinetic constants determined in the current work, we identified one more potential checkpoint for mRNA selection and completed a scheme of potential mRNA checkpoints during translation initiation. 


\section{Supplementary Information for Chapter 1.}

Supplementary table 1. Melting temperatures and Gibbs free energies of mRNA secondary structures predicted by Mfold for the mRNA constructs. The predicted alternative folding forms are indicated with numbers in brackets ((1), (2), (3)).

\begin{tabular}{|l|l|l|}
\hline & $\mathrm{T}_{\mathrm{m}}{ }^{\circ} \mathrm{C}$ & $\begin{array}{l}\Delta \mathrm{G}^{\circ}, \\
\mathrm{kcal} / \mathrm{mol}\end{array}$ \\
\hline m022 AUG (1) & 49.3 & -5.98 \\
\hline m022 AUG (2) & 51.6 & -5.6 \\
\hline m002 AUG (1) & 55.1 & -4.39 \\
\hline m002 AUG (2) & 41.8 & -3.53 \\
\hline m002 no AUG (1) & 46.7 & -5.38 \\
\hline m002 no AUG (2) & 47.7 & -5.07 \\
\hline m003 AUG (1) & 25.0 & -0.59 \\
\hline m003 AUG (2) & 24.2 & -0.52 \\
\hline m003 AUG (3) & 17.4 & -0.19 \\
\hline m003 no AUG (1) & 21.9 & -0.15 \\
\hline m003 no AUG (2) & 18.9 & 0.09 \\
\hline m003 no AUG (3) & 17.4 & 0.19 \\
\hline m6a & 96.3 & -15.31 \\
\hline & & \\
\hline
\end{tabular}


Supplementary Figure 1. mRNA structures predicted by Mfold at $20^{\circ} \mathrm{C}$. The predicted alternative folding forms of the same mRNA are indicated with numbers in brackets ((1), (2), (3).

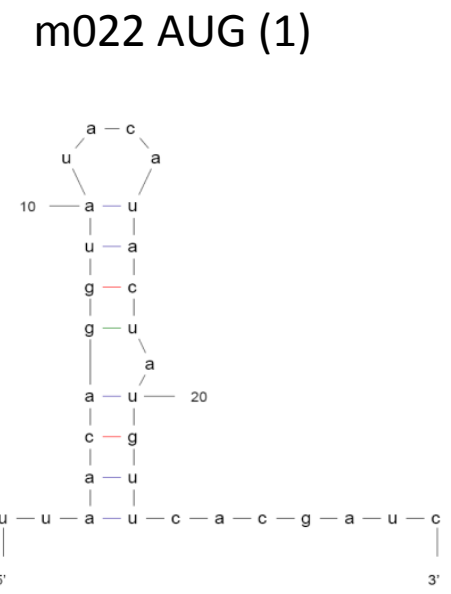

m002 AUG (1)

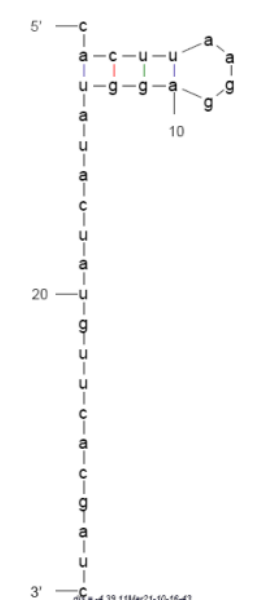

m002 no AUG (1)

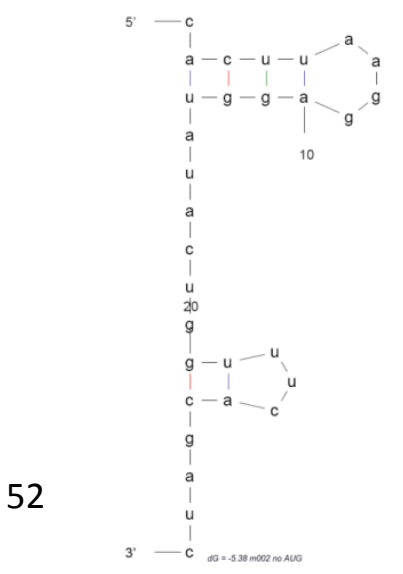

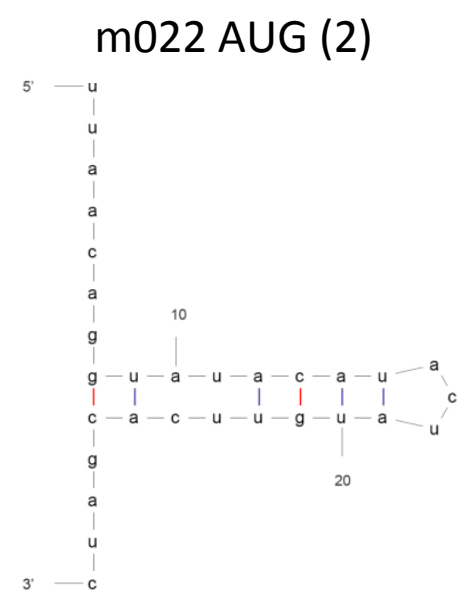

m002 AUG (2)

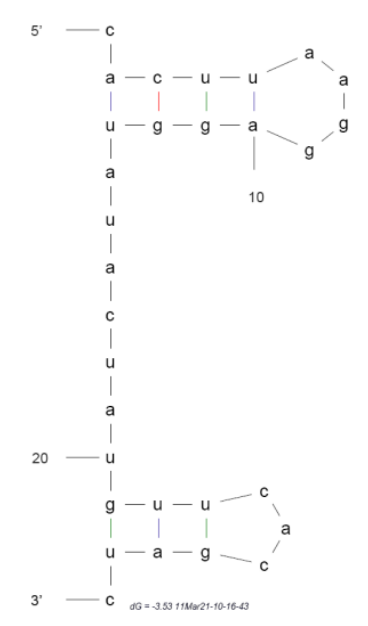

m002 no AUG (2)

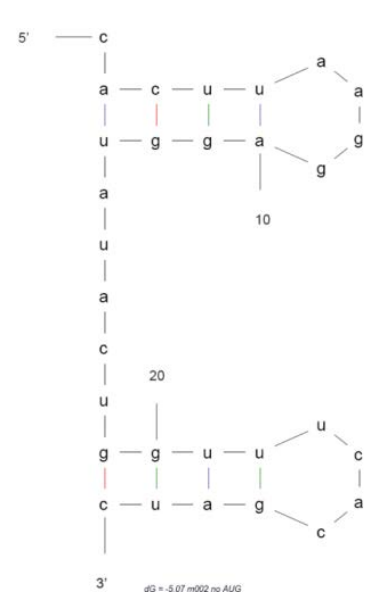


Supplementary Figure 1 (continuation). mRNA structures predicted by Mfold at $20^{\circ} \mathrm{C}$. The predicted alternative folding forms of the same mRNA are indicated with numbers in brackets ((1), (2), (3).
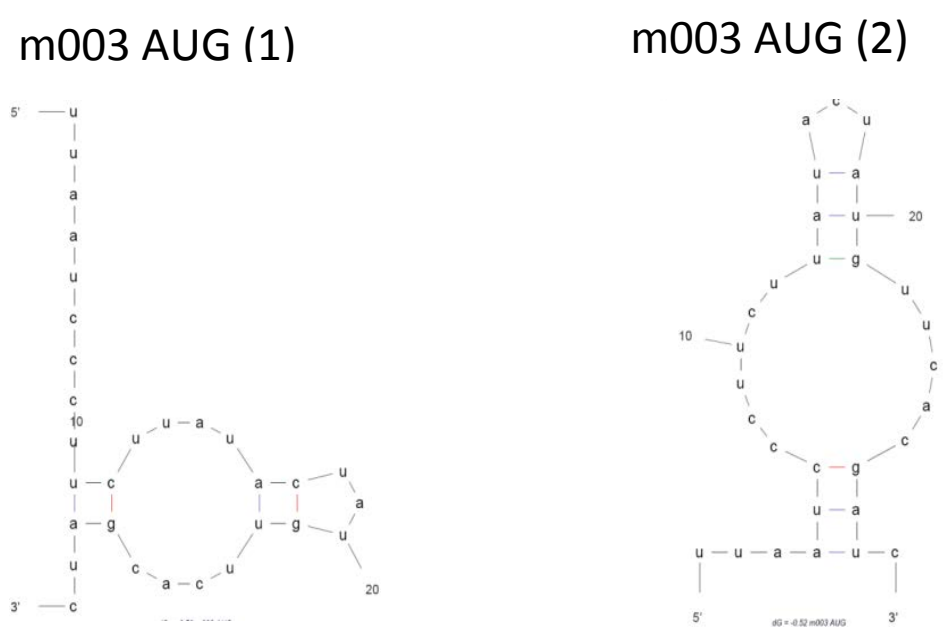

m003 AUG (3)

m003 no AUG (1)

m003 no AUG (2)

m003 no AUG (3)
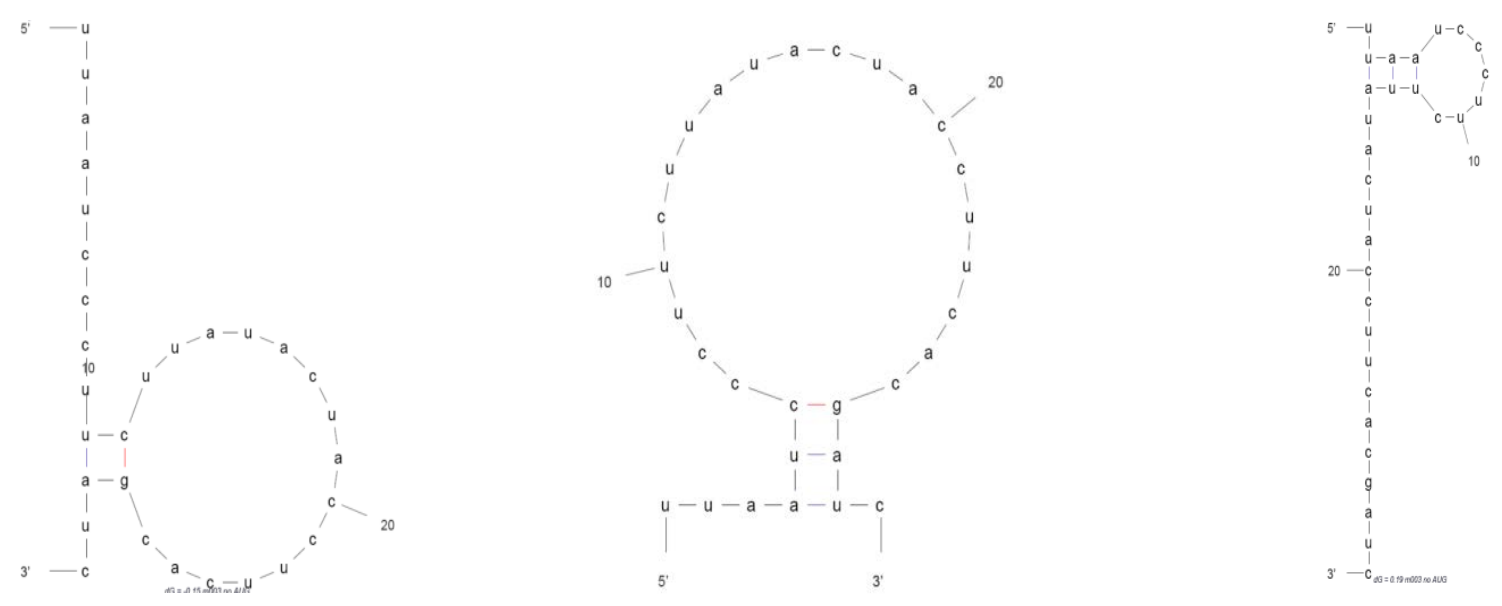

m6a

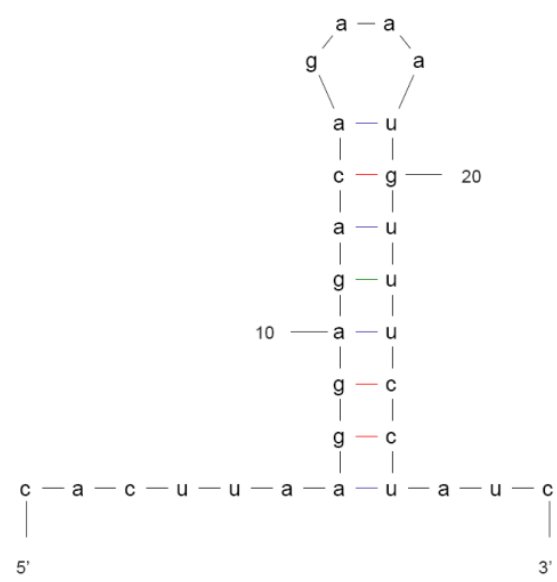




\section{Chapter 3. Kinetics of GTP hydrolysis by IF2.}

\section{Introduction.}

IF2 belongs to the superfamily of translational GTPases. It is the largest of the three prokaryotic initiation factors and is comprised of three domains: the N-terminal domain; the middle GTP-binding domain which has the typical structure of the GTP-binding domains and shares common motifs with the other translational GTPases; and the Cterminal domain which interacts with the CCA-end of fMet-tRNA ${ }^{\text {fMet }}$ (Roll-Mecak et al., 2000). IF2 binds GTP or GDP, as well as their analogs with the affinities in the $10-100$ $\mu \mathrm{M}$ range. Under the physiological conditions GDP concentration in the cell is very low, and thus the factor is expected to be present predominantly in the GTP-bound form (Pon et al., 1985). The intrinsic GTPase of IF2 is very low (Severini et al., 1991). However IF2 bound to the ribosome shows increased GTPase activity (Dubnoff and Maitra, 1972). During initiation complex formation, IF2 in the complex with GTP binds to the 30S PIC (Milón et al., 2012b), followed by the recruitment off Met-tRNA ${ }^{\text {fMet }}$ to IF2 (Milon 2010). Formation of the 30S IC as a results of start codon recognition and joining of the $50 \mathrm{~S}$ subunit to the 30S IC induces rapid GTP hydrolysis (Grigoriadou et al., 2007a; Tomsic et al., 2000). Subsequently IF2 undergoes conformational rearrangements (Grigoriadou et al., 2007a; Myasnikov et al., 2005), small and large ribosomal subunits move relative to one another (Marshall et al., 2009) and fMet-tRNA ${ }^{\text {fMet }}$ is positioned into the P site (La Teana et al., 1996).

Cryo-electron microscopy reconstructions of the $30 \mathrm{~S}$ subunit in complex with mRNA, IF1, IF2-GTP, and fMet-tRNA ${ }^{\text {fMet }}$ indicated that upon 50S subunit joining, the GTP-binding pocket of IF2 is placed in contact with the sarcin-ricin loop of the 23S rRNA of the 50S subunit, which triggers the GTPase of IF2 (Simonetti et al., 2008). These structural studies revealed that the GTP-binding domain of IF2 is positioned on the 50 S subunit in the same way as that of other ribosome-associated GTPases and suggested the conformational changes of IF2 that occur during the initiation. The factor was found in three distinct conformations: 1) after the association of the 50S subunit but before GTP hydrolysis (IF2-GTP), 2) after GTP hydrolysis (IF2-GDP-Pi), and 3) after the phosphate release (IF2-GDP) (Myasnikov et al., 2005; Simonetti et al., 2008). Given the sequence of events during late steps of translation initiation and the conformational changes of IF2 on the ribosome, it is likely that the first conformational rearrangement of IF2 may be related to the GTPase activation of IF2. The second conformational change of IF2 
possibly reflects stabilization of $70 \mathrm{~S} I \mathrm{C}$ and the third might represent PMet-tRNA $^{\text {fMet }}$ positioning in the $\mathrm{P}$ site (Grigoriadou et al., 2007a; Myasnikov et al., 2005; Simonetti et al., 2008).

Unlike small GTPases of the Ras family, IF2 does not function as a switch, which is active in the GTP-bound and inactive in the GDP-bound form (Tomsic et al., 2000). Nucleotide binding to IF2 is required for the $30 \mathrm{~S} \mathrm{IC} \mathrm{formation,} \mathrm{however} \mathrm{the} \mathrm{complex} \mathrm{is} \mathrm{formed} \mathrm{with}$ either GTP or GDP. On the other hand, GTP-bound IF2 has a higher affinity to the 30S subunit than IF2-GDP or IF2 alone (Pon et al., 1985). The question about the role of IF2 GTPase remained opened for a long time. Several functions have been attributed to the GTPase of IF2. It was suggested that GTP hydrolysis by IF2 might be required for proper fMet-tRNA $^{\text {fMet }}$ positioning in the $P$ site on the ribosome (Gualerzi and Pon, 1990). However, the presence of IF2-bound GTP was not necessary for the fMet-tRNA ${ }^{\text {fMet }}$ to react with the puromycin implying that also in the absence of GTP hydrolysis by IF2 fMet-tRNA $^{\text {fMet }}$ was properly positioned in the P site (La Teana et al., 1996). It was also proposed that GTP hydrolysis by IF2 is needed for IF2 to change conformation and be able to leave the ribosome as IF2 partially occupies the same binding site as EF-Tu and EF-G (La Teana et al., 2001). This hypothesis appeared to be supported by the observations that in the absence of GTP hydrolysis there was no progression of the ribosome to the elongation step (Luchin et al., 1999). It is important to note here that IF2 changes its conformation on the ribosome depending on being GTP or GDP bound. In the GTP-bound form, the IF2 is forming a contact with fMet-tRNA ${ }^{\text {fMet }}$, but in the GDP form this interaction is broken, bringing the IF2 into the "ready-to-leave" conformation (Myasnikov et al., 2005). Therefore, it is possible that GTP hydrolysis by IF2 may be required for the dissociation of the IF2 C-terminus from the fMet-tRNA ${ }^{\mathrm{fMet}}$. Yet another function for the GTPase function of IF2 was proposed by Marshall et al. (Marshall et al., 2009). Based on single molecule studies the authors conclude that IF2-catalyzed GTP hydrolysis promotes the rotation of the ribosomal subunits relative to one another from the so called "rotated state" to the "nonrotated", which controls the progression of ribosomes from initiation to elongation and through the elongation cycles.

Understanding of the functional role of the GTPase of IF2 is impeded by the uncertainties concerning its precise timing and molecular mechanism. In the present work the rate of GTP hydrolysis by IF2 was measured using a novel fluorescence-based assay. These measurements, in conjunction with the experiments on dissociation of IF1 and IF3, allowed for placing the GTPase reaction in the model of late events in translation initiation. 


\section{Materials and Methods.}

All reactions described in this chapter were carried out at $20^{\circ} \mathrm{C}$ in $\mathrm{TAKM}_{7}$ buffer $(50 \mathrm{mM}$ Tris $\mathrm{pH}$ 7.5, $70 \mathrm{mM} \mathrm{NH}_{4} \mathrm{Cl}, 30 \mathrm{mM} \mathrm{KCl}, 7 \mathrm{mM} \mathrm{MgCl}_{2}$ ) unless stated otherwise. The exact concentrations of the components of the reactions are given in the Results section as they vary in different experiments. Ribosomes, initiation factors and fMet-tRNA ${ }^{\text {fMet }}$ and mRNA were prepared as described (Milon et al., 2007).

\section{GTPase assay.}

\section{GTP-solutions.}

Mant-GTP was purchased from Jena Biosciences, Jena, Germany. [ $\left.{ }^{3} \mathrm{H}\right] \mathrm{GTP}$ (Sigma) or ${ }^{32}$ P]GTP (Hartmann Analytic) stock solutions were diluted with GTP (Roche) to the appropriate molar concentration and specific radioactivity (specific activity range was $2000 \mathrm{~m}-10000 \mathrm{dpm} / \mathrm{pmol})$.

\section{GTPase reaction.}

Two mixtures were prepared: one containing the $30 \mathrm{~S} \mathrm{IC} \mathrm{( \operatorname {mix }} 1$ ) and the second containing 50S subunit and GTP (mix 2). Mix 1 was prepared by mixing 30 S subunits, IF1, IF2, IF3, GTP, fMet-tRNA ${ }^{\text {fMet }}$ and mRNA in TAKM $_{7}$ buffer (exact concentrations are provided in the Results section) and incubating at $37^{\circ} \mathrm{C}$ for $30 \mathrm{~min}$. Subsequently the mix was kept in ice for $10 \mathrm{~min}$. Mix 2 was prepared from the 50S subunits and GTP at appropriate concentrations in the $\mathrm{TAKM}_{7}$ buffer and kept in ice before use.

The fast kinetics of IF2 GTPase reaction was measured using quench-flow apparatus (KinTek). The reaction was started by mixing 30S IC with 50S subunit in the apparatus. The instrument triggers rapid mixing of equal volumes $(14 \mu \mathrm{l})$ of the respective solutions in the mixing chamber. The reaction is then incubated for defined times (ranging from 5 $\mathrm{ms}$ to any desired time) before stopping with a quencher solution. For the GTPase experiments, $50 \%$ formic acid was used as a quencher (Rodnina et al., 1999). Slow GTPase reactions were performed in the same way, except that the solutions were mixed manually. 
After quenching, the reaction mixtures were centrifuged for $20 \mathrm{~min}$ at $4^{\circ} \mathrm{C}(13000 \mathrm{rpm})$ to eliminate protein and rRNA precipitates. Guanosine nucleotides, small molecules, tRNA and mRNA are soluble at acidic conditions and remain in solution. The supernatant after centrifugation was transferred to a new tube. $\mathrm{pH}$ of the sample was increased to 4 -4.5 by adding the appropriate volume of $5 \mathrm{~N} \mathrm{KOH}$. The samples were loaded onto High Performance Liquid Chromatography (HPLC)(if mant-GTP or $\left[{ }^{3} \mathrm{H}\right]$ GTP were used in the reaction) or analyzed by TLC (if [ ${ }^{32}$ P]GTP was used in the reaction).

\section{Separation of guanine nucleotides by HPLC.}

GTP was separated from GDP using an anion exchange HPLC column Nucleosil 4000-7 PEI. The chromatography material of the column is silica, particle size $7 \mu \mathrm{m}$, pore size $4000 \AA$, and covalently bonded polyethyleneimine network which works as a weak basic anion exchanger. The negatively charged phosphate groups of guanine nucleotides are reversely bound to the positively charged groups of the resin from where they can be eluted by either increasing the ionic strength of the eluent. Two buffers were used for separating GTP from GDP: buffer A (10 mM Tris pH 8.0) and buffer $B(20 \mathrm{mM}$ Tris $\mathrm{pH}$ 8.0; $2 \mathrm{M} \mathrm{NaCl}$ ). For separating mant-GTP from mant-GDP $10 \%$ acetonitrile was added to buffer $A$ and buffer $B$. The column was first equilibrated by salt-free buffer A. After loading of the sample, the column was washed with 3 bed volumes of buffer $A$ to remove the unbound compounds. Nucleotides were eluted by an adapted gradient of from $30 \%$ to $100 \%$ of buffer B. At the end of the gradient, the column is washed by $100 \%$ buffer $B$ for $1 \mathrm{~min}$ and then with buffer $A$ for $1 \mathrm{~min}$. The flow rate was $2 \mathrm{ml} / \mathrm{min}$; total running time including the washing steps $11 \mathrm{~min}$. The fluorescence of mant-GTP was analyzed by flow-through fluorescent detector (2475 Multi $\lambda$ Fluorescence Detector, Waters). For the analysis of $\left[{ }^{3} \mathrm{H}\right]$-labeled nucleotides, $0.5 \mathrm{ml}$ HPLC fractions were collected. The radioactivity in the fractions was determined using liquid-liquid scintillation counting: $2 \mathrm{ml}$ of scintillation cocktail Lumasafe Plus (Perkin Elmer) was added to each fraction.

\section{Data analysis.}

Mant-GTP. After separation of mant-GTP and mant-GDP on the HPLC, two distinct peaks can be detected in the chromatogram. The chromatograms were analyzed and the area of the fluorescent peaks was quantified using the Empower 3 software (Waters). 
[3H]GTP. After separation of GTP and GDP by HPLC, fractions were collected and position of peaks identified by radioactivity counting. The total amount of radioactivity associated to $\left[{ }^{3} \mathrm{H}\right] \mathrm{GTP}$ and $\left[{ }^{3} \mathrm{H}\right] \mathrm{GDP}$ peaks was quantified.

The area of the peak corresponding to fluorescent nucleotide or radioactive nucleotide was quantified in relative fluorescence units or in units of radioactivity (dpm) respectively. The ratio of the GDP peak to the sum of GDP and GTP peaks was calculated yielding the percentage of GTP hydrolyzed in the reaction. As only IF2 bound to the $30 \mathrm{~S}$ IC can hydrolyze GTP, dividing the amount of hydrolyzed GTP by the amount of the 30S IC in the reaction gives the amount of GTP hydrolyzed per 30S IC. Thus, we obtain the percentage of GTP hydrolyzed by IF2 per 30S IC at every time point measured. These values are plotted as a function of time. The data were processed and evaluated by single- or double-exponential fitting using the Prism (Graphpad Software).

\section{Thing layer chromatography (TLC).}

When $\left[\gamma^{-32} \mathrm{P}\right]$ GTP was used in the GTPase reaction, the reaction products were separated on the TLC. TLC was performed on the polyethyleneimine-cellulose plates. The plates were prewashed with water and air-dried. $2 \mu \mathrm{l}$ of the samples after GTPase reaction were loaded and the plate was air-dried. The separation of $\left[\mathrm{\gamma}^{-32} \mathrm{P}\right] \mathrm{GTP}$ from $\left[{ }^{32} \mathrm{P}\right] \mathrm{Pi}$ was carried out using $0.5 \mathrm{M} \mathrm{KH}_{2} \mathrm{PO}_{4}, \mathrm{pH} 3.5$, as a mobile phase. After the separation the plate was air-dried and visualized on the Fuji Phosphorimager FLA7000. The intensity of the bands was quantified using ImageGauge software (Fuji). The data was processed further as described for mant-GTP.

\section{Measuring mant-GTP fluorescent change in the stopped-flow.}

Kinetic experiments were performed in the SX-20MV stopped-flow apparatus (Applied Photophysics, Leatherhead, UK). Experiments were carried out by rapidly mixing equal volumes ( $60 \mu \mathrm{l}$ each) of reactants at $20^{\circ} \mathrm{C}$, containing 30S IC with mant-GTP in one of the syringes and large excess (1250 times) of non-labeled GTP. Mant was excited at $290 \mathrm{~nm}$ and fluorescence change upon its dissociation from the $30 \mathrm{~S} \mathrm{IC} \mathrm{was} \mathrm{monitored} \mathrm{using} \mathrm{a}$ cut-off filter for $408 \mathrm{~nm}$ (KV408, Schott). The average of 7-10 repetitions was taken to decrease the fluorescent signal error. The apparent rate constant was determined by fitting the traces with single exponential equation $Y=A 0+A 1 * \exp (-k 1 * t)$ where $A 0$ 
corresponds to the final amplitude, A1 to amplitude of the corresponding phase and $\mathrm{k} 1$ to the apparent rate.

\section{Results.}

Separation of the guanine nucleotides on the anionic exchange HPLC column Nucleosil 4000-7 PEI.

To establish conditions for the separation of GTP from GDP, we first tested the separation of equal amounts of radioactive $\left[{ }^{3} \mathrm{H}\right]$ GTP and $\left[{ }^{3} \mathrm{H}\right]$ GDP (20 nmoles each) with the Nucleosil 4000-7 PEI column (Fig. 3.1). To detect the nucleotides, optical density of the eluate was measured at $\lambda=260 \mathrm{~nm}$, as well as the radioactivity in the HPLC fractions. Various conditions for the separation were tested, including composition of the elution buffer, different elution times and velocity of elution. To distinguish which of the elution peaks corresponds to the particular nucleotide, chromatography runs with $\left[{ }^{3} \mathrm{H}\right] \mathrm{GTP}$ alone or $\left[{ }^{3} \mathrm{H}\right]$ GDP alone were performed. Clear separation of the two nucleotides was achieved with GDP eluting earlier (ca. 9.5 min retention time) and GTP eluting later (ca. 12 min retention time) (Fig 3.1). When the radioactivity in the HPLC fractions was measured, the location of the peaks in the chromatogram corresponded to that in the optical density chromatograms. The fractions containing $\left[{ }^{3} \mathrm{H}\right]$ GDP and $\left[{ }^{3} \mathrm{H}\right] \mathrm{GTP}$ were separated by one fraction due to a relatively large volume of fractions collected, which is however sufficient for quantification of the peaks.
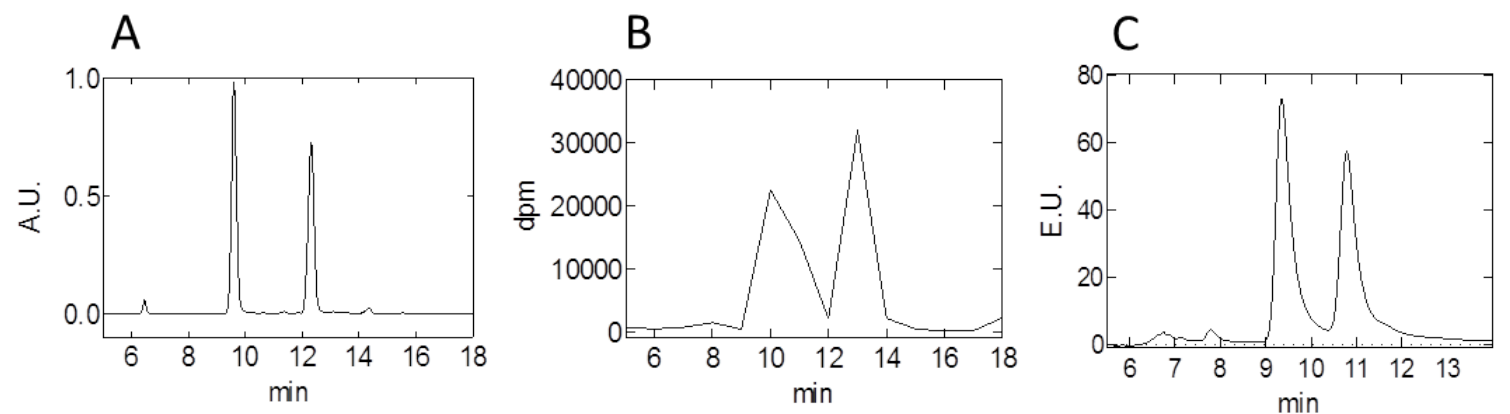

Figure 3.1 Elution profiles of GDP and GTP from the Nucleosil 4000-7 PEI (A) absorbance at 260 $\mathrm{nm}$; (B) radioactivity of tritium labeled nucleotides counted in the collected HPLC fractions; (C) fluorescence of mant-derivatives of guanine nucleotides (excitation $360 \mathrm{~nm}$, emission 448 $\mathrm{nm})$. 
The best conditions for separating GDP from GTP were found to be the following. Elution buffer A contained $10 \mathrm{mM}$ Tris- $\mathrm{HCl}$ pH 8.0; elution buffer B contained $20 \mathrm{mM}$ Tris- $\mathrm{HCl} \mathrm{pH}$ $8.0 ; 2 \mathrm{M} \mathrm{NaCl}$. Elution speed was $2 \mathrm{ml} / \mathrm{min}$.

Studying the kinetics of single turnover GTPase reactions requires precise quantification of the amount of GTP hydrolyzed within time periods in a milliseconds range. The amount of reaction product formed in these time intervals cannot be detected by measuring the nucleotide absorbance at $260 \mathrm{~nm}$ as it is below the sensitivity limit of the flow-through detector. Quantification of the tritium labeled reaction products also has a disadvantage of having long handling times and using additional materials such as vials and scintillation cocktail. Therefore, we additionally modified the GTPase assay to use a fluorescent derivative of GTP (Fig. 3.2). Mant-GTP is a nucleotide analog where either the 2'- or the 3'-hydroxy group of ribose has been esterified by the fluorescent methylisatoic acid. Mant-label is relatively small compared to other fluorescent labels. The replacement of GTP with this fluorescent analog was shown not to alter the rate of GTP hydrolysis by IF2 (see below).

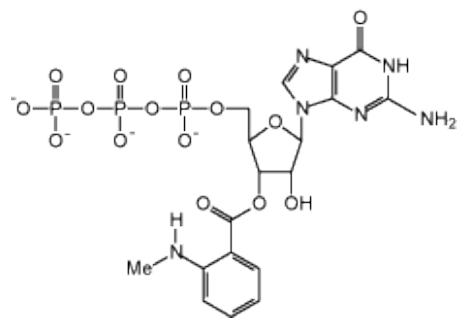

Figure 3.2 Mant-GTP - fluorescent GTP derivative used in the study.

Mant-GDP and mant-GTP were found to be eluted from the Nucleosil 4000-7 PEI later than non-fluorescent nucleotides when same eluting conditions were used. The retention time was close to the end of the gradient, where it was not possible to precisely quantify the amount of mant-GTP. The protocol was further optimized for separation of the mant-derivatives of the nucleotides by addition of $10 \%$ acetonitrile to the elution buffers $A$ and $B$, which shifted the retention times of mant-GDP and mantGTP to ca. $5.5 \mathrm{~min}$ and $7 \mathrm{~min}$ respectively. Due to the increase in solvent hydrophobicity, sharper and more symmetrical elution peaks were obtained, providing a reliable signal to quantify GTP hydrolysis. 


\section{Validation of the GTPase assay.}

GTPase reaction was carried out as described in "Materials and Methods" section. The GTPase activity of IF2 was triggered by mixing the 30S IC with the 50S subunits. The reaction was quenched with 50\% formic acid (Rodnina et al., 1999). At such acidic conditions, the ribosomes and translation factors are inactivated, and spontaneous hydrolysis of GTP does not occur. The proteins and rRNA were removed by centrifugation. In the remaining supernatant the $\mathrm{pH}$ value was increased to ca. 4.5 by adding $\mathrm{KOH}$ as it is not possible to apply samples with a $\mathrm{pH}<2$ to the HPLC column. The reaction products are separated on the ion-exchange column using the protocol described before. Mant-labeled nucleotides were detected by the flow through detector; for tritium-labeled nucleotides the fractions after the HLC run were collected and radioactivity of the fractions measured. The separation pattern for GDP and GTP after the GTPase reaction looked the same as when separating standard nucleotide solutions. The relative sizes of the GTP and GDP peaks depended on the incubation time of the GTPase reaction or the concentration of the GTPase activator (50S subunit), indicating that the assay is suitable for measuring fast kinetics of the GTPase reaction.
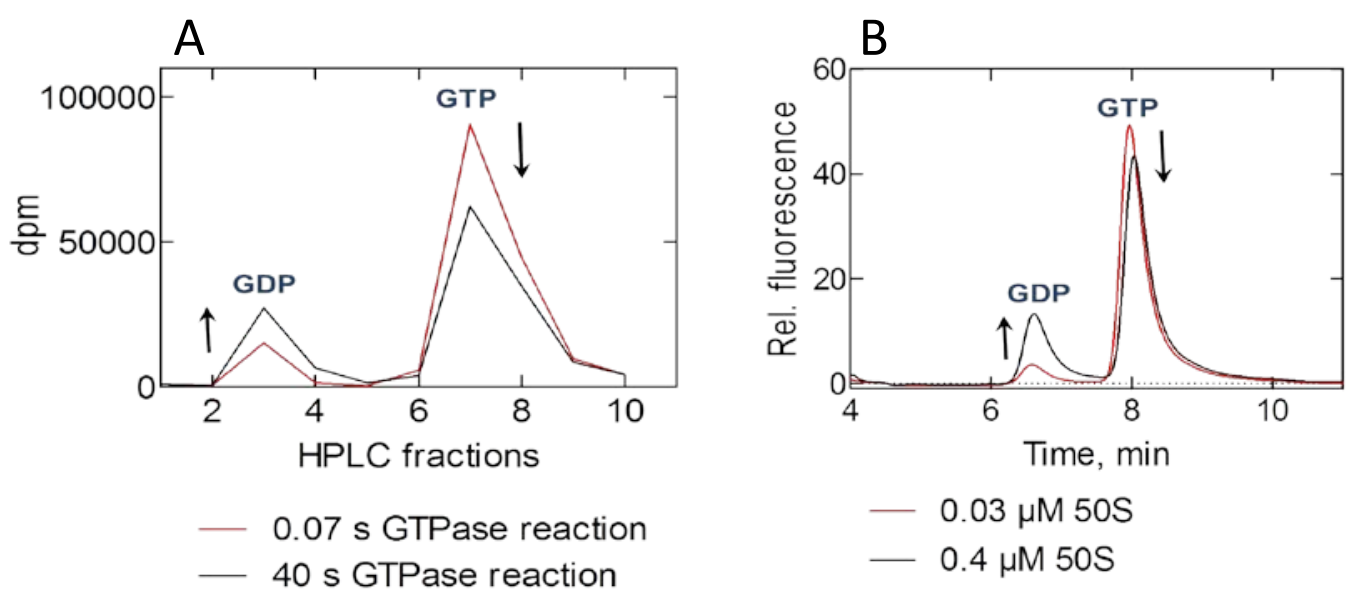

Figure 3.3 (A) The separation pattern of $\left[{ }^{3} \mathrm{H}\right] \mathrm{GTP}$ and $\left[{ }^{3} \mathrm{H}\right] \mathrm{GDP}$ depending on the incubation time of the GTPase reaction. (B) The separation pattern of mant-GTP and mant-GDP depending on the concentration of the $50 \mathrm{~S}$ subunit in the GTPase reaction.

\section{Rapid kinetic measurements of GTP hydrolysis by IF2 using [ $\left.{ }^{32} \mathrm{P}\right] \mathrm{GTP},\left[{ }^{3} \mathrm{H}\right] \mathrm{GTP}$ or mant- GTP.}

When establishing the GTPase assay, we performed a number of experiments where the rates of GTPase reaction with $\left[{ }^{3} \mathrm{H}\right.$ ]GTP and mant-GTP were compared to exclude the possibility of artifacts introduced in the measurement by fluorescent nucleotide 
derivative. For control, GTPase reaction utilizing $\left[{ }^{32} \mathrm{P}\right]$ GTP and separating the reaction products on TLC was also performed. The latter method was used previously in the field for measuring the velocity of GTP hydrolysis by IF2 (Tomsic et al., 2000).

The GTPase reactions were carried out as described in Materials and Methods section. The products of the GTPase reactions were separated either on HPLC (for $\left[{ }^{3} \mathrm{H}\right] \mathrm{GTP}$ and mant-GTP) or on TLC (for [ $\left.{ }^{32} \mathrm{P}\right] \mathrm{GTP}$ ). The amounts of reaction products were quantified and the percentage of GTP hydrolyzed by IF2 relative to the total amount of 30S IC was plotted against time (Fig. 3.4). In all three versions of the GTPase reaction, independently on the GTP analog used, an exponential phase can be distinguished followed by the slow linear phase. The burst phase corresponds to GTP hydrolysis by IF2, the slope may represent multiple turnover of GTP on IF2. In the reaction where radioactive nucleotides were used the initial level of GDP is high, possibly due to the unspecific GTP hydrolysis which happens during the storage, or some contaminations that move together with $\mathrm{Pi}$ on the TLC plates. The reaction where mant-GTP was used shows the lowest initial level of GDP, but the stoichiometry of the reaction per 30S IC is only $30 \%$, compared to about $60 \%$ found with $\left[{ }^{3} \mathrm{H}\right] \mathrm{GTP}$. The rates of the first round of GTP hydrolysis by IF2 obtained in these experiments are similar (Table 3.1). The small differences observed are insignificant and may be caused by minor differences sample handling.
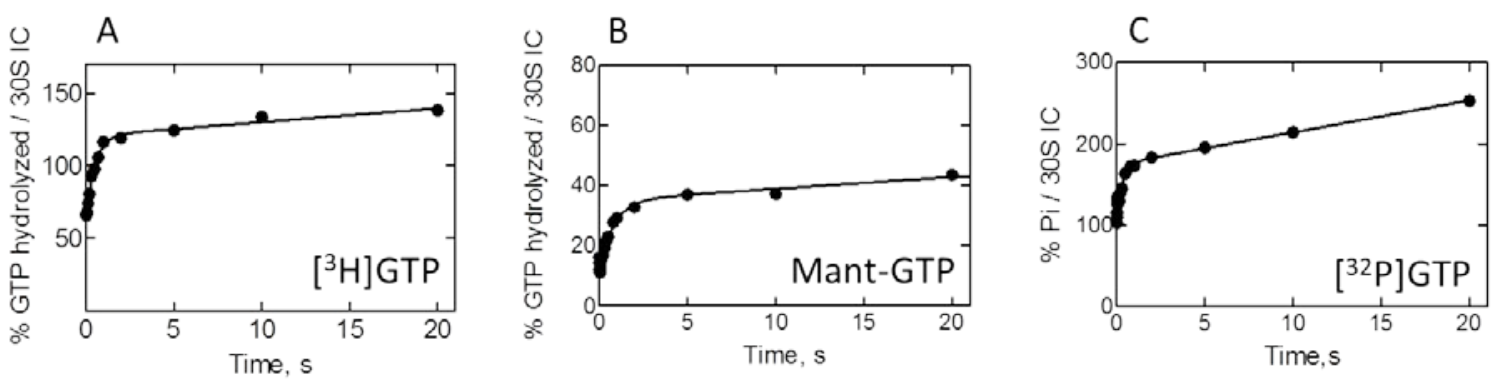

Figure 3.4 GTP hydrolysis by IF2 using (A) $\left[{ }^{3} \mathrm{H}\right] \mathrm{GTP}$; (B) mant-GTP; (C) $\left[{ }^{32} \mathrm{P}\right] \mathrm{GTP}$. (A) and (B) - GTPase reaction products were separated on the HPLC, (C) - GTPase reaction products were separated by TLC, as described in Materails and Methods. The final concentrations of the components were as follows: (A) $0.15 \mu \mathrm{M}$ 30S subunits; $0.45 \mu \mathrm{M}$ IF1; $0.3 \mu \mathrm{M}$ IF2, $0.3 \mu \mathrm{M}$ IF3; $0.3 \mu \mathrm{M}$ fMet-tRNA ${ }^{\text {fMet; }}$; $1 \mu \mathrm{M}$ mRNA; $2 \mu \mathrm{M}\left[{ }^{3} \mathrm{H}\right] \mathrm{GTP}$; GTP hydrolysis was triggered by $0.45 \mu \mathrm{M} 50 \mathrm{~S}$ subunits together with $2 \mu \mathrm{M}\left[^{3} \mathrm{H}\right] \mathrm{GTP}$; (B) $0.2 \mu \mathrm{M}$ 30S subunits; $0.4 \mu \mathrm{M}$ IF1; $0.25 \mu \mathrm{M}$ IF2; $0.4 \mu \mathrm{M}$ IF3; $0.5 \mu \mathrm{M}$ fMet-tRNA ${ }^{\text {fMet }}$; $1 \mu \mathrm{M}$ mRNA; $1 \mu \mathrm{M}$ mant-GTP; GTP hydrolysis was triggered by $0.6 \mu \mathrm{M}$ 50S subunits together with $1 \mu \mathrm{M}$ mant-GTP; (C) $0.2 \mu \mathrm{M}$ 30S subunits; $0.5 \mu \mathrm{M}$ IF1; $0.25 \mu \mathrm{M}$ IF2; $0.5 \mu \mathrm{M}$ IF3; $0.4 \mu \mathrm{M}$ fMet-tRNA ${ }^{\text {fMet }}$; $0.6 \mu \mathrm{M}$ mRNA; $18 \mu \mathrm{M}\left[{ }^{32} \mathrm{P}\right] \mathrm{GTP} ;$ GTP hydrolysis was triggered by $0.6 \mu \mathrm{M} 50 \mathrm{~S}$ subunits together with 
Table 3.1 The rates of GTP hydrolysis by IF2 obtained using different GTP analogs.

\begin{tabular}{|l|l|}
\hline GTP analog & $k_{a p p}, \mathrm{~s}^{-1}$ \\
\hline$\left[{ }^{3} \mathrm{H}\right]$ GTP & $2.0 \pm 0.2$ \\
\hline Mant-GTP & $1.2 \pm 0.2$ \\
\hline$\left[{ }^{32} \mathrm{P}\right]$ GTP & $2.9 \pm 0.7$ \\
\hline
\end{tabular}

\section{Rapid kinetic measurements of mant-GTP hydrolysis by IF2.}

The rate of rapid GTP hydrolysis by IF2 was measured using the GTPase assay utilizing fluorescent mant-GTP. GTP hydrolysis was measured in the time periods ranging from 6 $\mathrm{ms}$ to $60 \mathrm{~s}$. The kinetics of GTP hydrolysis by IF2 is described by the single exponential equation with a slope (Fig. 3.5). The rate of the fast phase of GTPase is essentially independent from the 50S subunit concentration arriving (Fig. 3.7). It was previously shown that the association rate of the 30S IC joining with the 50S subunit is linearly dependent on the $50 \mathrm{~S}$ concentration with the association rate constant about $10 \mu \mathrm{M}^{-1} \mathrm{~s}^{-1}$ (Antoun et al., 2006a; Milon et al., 2008). Taking into account that $50 \mathrm{~S}$ subunit is the activator of GTP hydrolysis by IF2 and that we see no dependence of the rate of GTPase reaction on the $50 \mathrm{~S}$ subunit concentration, one can suggest that the rate limiting step is either GTPase activation step or the GTP hydrolysis itself.
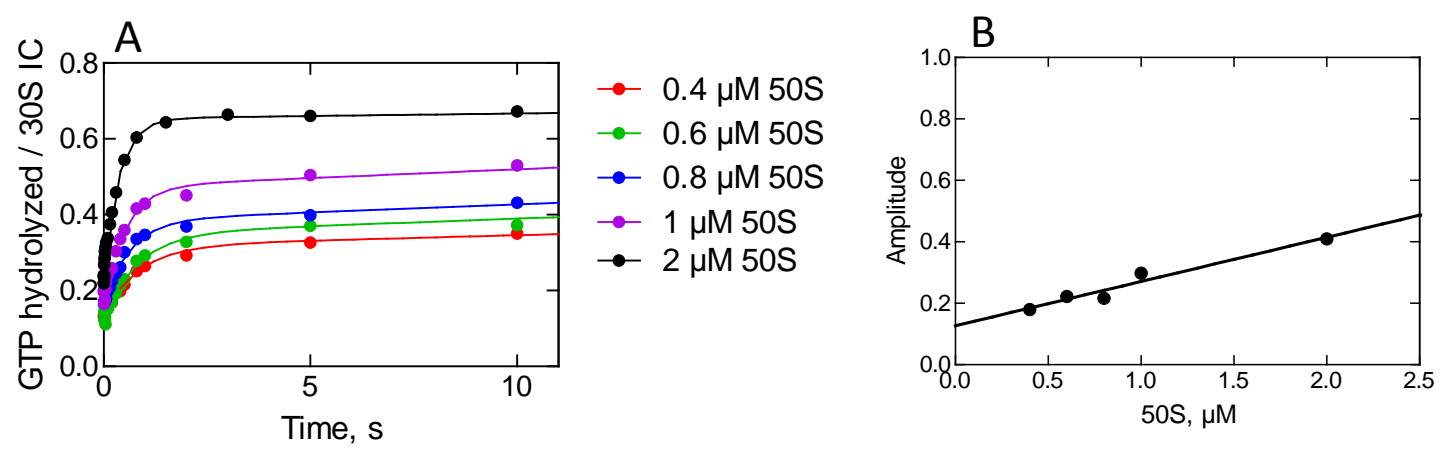

Figure 3.5 (A) Dependence of mant-GTP hydrolysis by IF2 on the 50 S subunits concentration. The following final concentrations were used for the reaction: $0.2 \mu \mathrm{M}$ 30S subunit; $0.4 \mu \mathrm{M}$ IF1; $0.25 \mu \mathrm{M}$ IF2; $0.4 \mu \mathrm{M}$ IF3; $0.4 \mu \mathrm{M}$ fMet-tRNA ${ }^{\text {fMet }} ; 0.8 \mu \mathrm{M}$ mRNA, $1 \mu \mathrm{M}$ mant-GTP. The reaction was triggered by rapid mixing with various concentrations of $50 \mathrm{~S}$ subunit and $1 \mu \mathrm{M}$ mant-GTP to avoid GTP concentration change. The following concentrations of $50 \mathrm{~S}$ subunit were used for titration: $0.4 \mu \mathrm{M} ; 0.6 \mu \mathrm{M} ; 0.8 \mu \mathrm{M} ; 1 \mu \mathrm{M} ; 2 \mu \mathrm{M}$. (B) Amplitude dependence from the $50 \mathrm{~S}$ subunit concentration. 


\section{IF1 and IF3 do not affect IF2 catalyzed GTP hydrolysis.}

To check the influence of the components of the 30 S IC on the GTPase activity of IF2, we measured the rate of GTP hydrolysis by IF2 in the complex where IF1 or IF3 were omitted (Fig. 3.6). The apparent rate constants of GTP hydrolysis by IF2 in the absence of IF1 or IF3 at different concentrations of the 50S subunits were calculated from the time courses of reactions by exponential fitting. The difference in the amplitudes reflects rather the efficiency of 30S IC formation in the given conditions. The dependence of the apparent rate constant on the concentration of the 50S subunit is hyperbolic, whereas the concentration dependence of the apparent rate constants of subunit joining is linear and does not reach saturation (Milon et al., 2008). These observations suggest that the subunit association is a bimolecular reaction that precedes the GTPase activation and GTP hydrolysis by IF2. The rate of the GTPaser reaction is slightly higher in the absence of IF1 or IF3 as the rate of the 50S subunit association is three times higher in the absence of IF1 and 5 times higher in the absence of IF3 compared to the complete 30S IC. The rate of GTPase reaction reaches saturation at the $2 \mu \mathrm{M}$ concentration of the $50 \mathrm{~S}$ subunit. It is also possible that some processes after subunit joining are rate limiting for the GTPase reaction, for example structural rearrangements in the 305 initiation complex upon 50 S subunit binding or the GTPase activation of IF2.
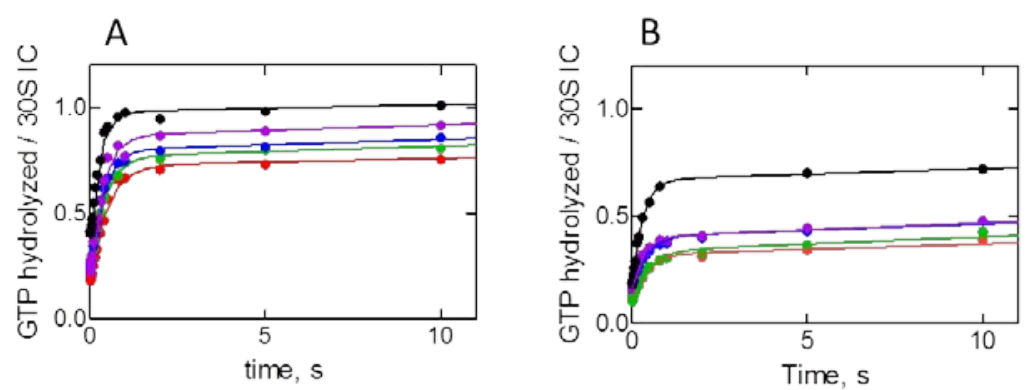

$$
\begin{aligned}
& \rightarrow 0.4 \mu \mathrm{M} 50 \mathrm{~S} \\
& \rightarrow 0.6 \mu \mathrm{M} 50 \mathrm{~S} \\
& \rightarrow 0.8 \mu \mathrm{M} 50 \mathrm{~S} \\
& \rightarrow 1 \mu \mathrm{M} 50 \mathrm{~S} \\
& \rightarrow 2 \mu \mathrm{M} 50 \mathrm{~S}
\end{aligned}
$$
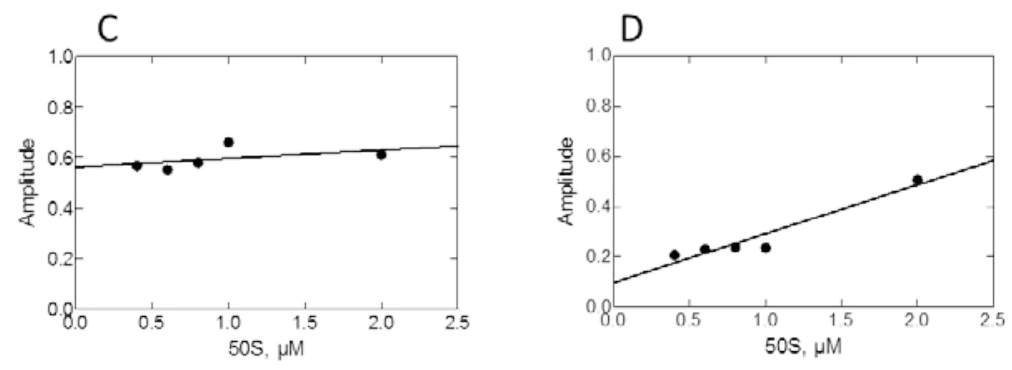

Figure 3.6 Mant-GTP hydrolysis by IF2 in the absence of (A) IF1; (B) IF3. The same procedure was used for forming 30 S IC without IF1 or IF3 as for the complete 30S IC formation. The following final concentrations of the components were used: $0.2 \mu \mathrm{M}$ 30S subunits; $0.4 \mu \mathrm{M}$ IF1 (where applicable); $0.25 \mu \mathrm{M}$ IF2; $0.4 \mu \mathrm{M}$ IF3 (where applicable); $0.4 \mu \mathrm{M}$ fMet-tRNA ${ }^{\text {fMet }}$; $0.8 \mu \mathrm{M}$ mRNA; 1 $\mu \mathrm{M}$ mant-GTP. The GTPase reaction was triggered by mixing $30 \mathrm{~S} \mathrm{IC}$ with $50 \mathrm{~S}$ subunit of various concentrations with $1 \mu \mathrm{M}$ mant-GTP. Concentrations of 50S subunit used were: $0.4 \mu \mathrm{M} ; 0.6 \mu \mathrm{M}$; $0.8 \mu \mathrm{M} ; 1 \mu \mathrm{M} ; 2 \mu \mathrm{M}$. (C), (D) - amplitude dependence on the 50S subunit concentration of $A$ and $B$, respectively. 


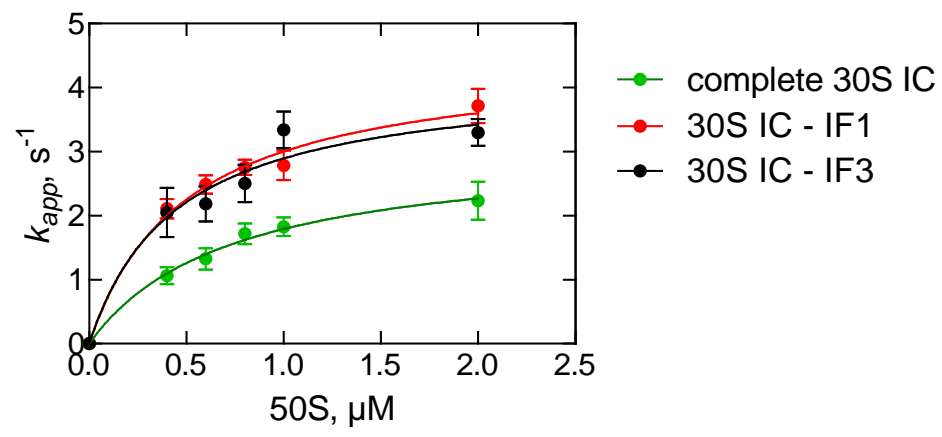

Figure 3.7 The apparent rate constants of GTP hydrolysis by IF2 plotted against the concentration of the $50 \mathrm{~S}$ subunit.

Table 3.2 Evaluation of the maximal velocity of GTP hydrolysis by IF2.

\begin{tabular}{|l|l|}
\hline & $\mathrm{V}_{\max } \mathrm{s}^{-1}$ \\
\hline complete 30S IC & 3.7 \\
\hline 30S IC lacking IF1 & 3.2 \\
\hline 30S IC lacking IF3 & 3.8 \\
\hline
\end{tabular}

\section{Rapid GTP hydrolysis by IF2 is triggered only in the complete $30 \mathrm{~S} \mathrm{IC.}$}

It has been shown that rapid 70S IC formation can occur only when a canonical 30S IC has been fully formed (Milon et al., 2008). The lack of initiator tRNA or mRNA causes slower association of the $50 \mathrm{~S}$ subunit with the $30 \mathrm{~S} \mathrm{IC.} \mathrm{The} \mathrm{GTPase} \mathrm{activity} \mathrm{of} \mathrm{IF2} \mathrm{is}$ thought to be triggered by the interaction with the sarcin loop in the $50 \mathrm{~S}$ subunit. Thus, one may expect that even in those cases where subunit association is slowed down, IF2 should hydrolyze GTP, albeit at slower rates.

To test the requirement for the complete 30 S IC, we performed an experiment where fMet-tRNA $^{\text {fMet }}$ was omitted. Two similar GTPase reactions were set up - with and without tRNA (Fig. 3.8). In the case of the complete $30 \mathrm{IC}$, the amount of $\left[{ }^{3} \mathrm{H}\right] \mathrm{GTP}$ hydrolyzed is increasing with time which corresponds to multiple GTP hydrolysis rounds. In the reaction with $\mathrm{fMet}_{\mathrm{TRNA}} \mathrm{fMet}$ omitted, there is no appreciable GTPase activity 
triggered by the binding of the $50 \mathrm{~S}$ subunit. The association rate constant of the $50 \mathrm{~S}$ subunit was shown to be about $10 \mu \mathrm{M}^{-1} \mathrm{~s}^{-1}$ (Antoun et al., 2006a; Milon et al., 2008). This suggests that in the time range where IF2 GTPase was measured all 50 S subunits should have been bound to the $30 \mathrm{~S}$ complexes. So it is not only the binding of the $50 \mathrm{~S}$ subunit, but also the presence of the fMet-tRAN ${ }^{\mathrm{fMet}}$, which is required for activation of the GTPase function of IF2. Most likely when the 30 S IC is incomplete, IF2 is present in the complex in a conformation which is insensitive to GTPase activation.

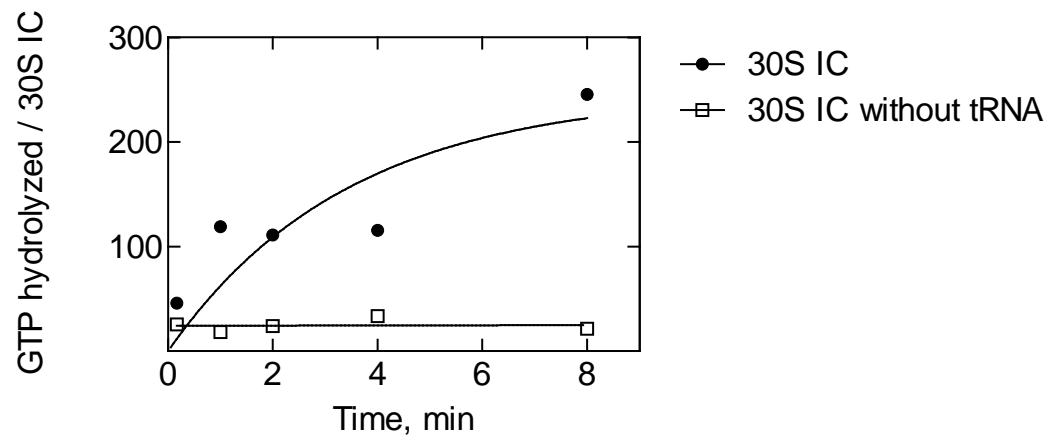

Figure 3.8 IF2 GTPase reaction on complete 30S IC (0.5 $\mu \mathrm{M}$ 30S subunits; $1 \mu \mathrm{M}$ IF1; $1 \mu \mathrm{M}$ IF2; $1 \mu \mathrm{M} \mathrm{IF3} ; 20 \mu \mathrm{M}$ [ $\left.{ }^{3} \mathrm{H}\right] \mathrm{GTP} ; 0.8 \mu \mathrm{M} 002$ mRNA and $0.8 \mu \mathrm{M}$ fMet-tRNA ${ }^{\mathrm{fMet}}$ ) and on the 30S PIC without fMet-tRNA ${ }^{\text {fMet }}$. The reaction was started manually by adding the $50 \mathrm{~S}$ ribosomes $(1 \mu \mathrm{M})$ to the mixture.

\section{Increasing mant-GTP concentration does not alter the rate of GTP hydrolysis by IF2.}

In the GTPase experiments described above the concentration of the reaction substrate was relatively low ( $1 \mu \mathrm{M}$ mant-GTP). Taken into consideration the affinity of IF2 to GTP $(10-40 \mu \mathrm{M})$ (Hauryliuk et al., 2009; Milon et al., 2006; Pon et al., 1985) and the concentrations used in the reaction, it was possible that the rate of GTP that we observe is in fact limited by GTP binding to the 30S IC. Therefore we determined the rate of IF2 catalyzed GTP hydrolysis at increasing concentrations of GTP (Fig 3.9).

The rate of the multiple turnover GTP hydrolysis is increasing with increasing GTP concentration which is expected. The rate of the fast phase of GTP hydrolysis is independent of the GTP concentration (Fig. 3.9 B). This means that GTP is bound to the 30S IC even al the lower GTP concentration used, and we observe indeed the rapid kinetics of GTP hydrolysis by IF2. This also supports the previously made observation that GTP is stabilized on IF2 upon the interaction of IF2 with the 30 S (Antoun et al., 2003) as free IF2 was shown to have relatively low affinity to GTP (Milon et al., 2006). 
Moreover, we confirmed the fact that GTP has different affinity to free IF2 and 30Sbound IF2 by the stopped-flow experiment using the fluorescent mant-GTP. We monitored the fluorescence change of mant-GTP upon dissociation from either free IF2 or IF2 bound to the 30S IC (Fig. 3.10). In both cases the traces were fitted by single exponential equation yielding the dissociation rate constants of $0.02 \pm 0.01$ for 30S IC (red trace in fig. 3.10) and $3.4 \pm 0.2$ for free IF2 (blue trace in fig. 3.10).
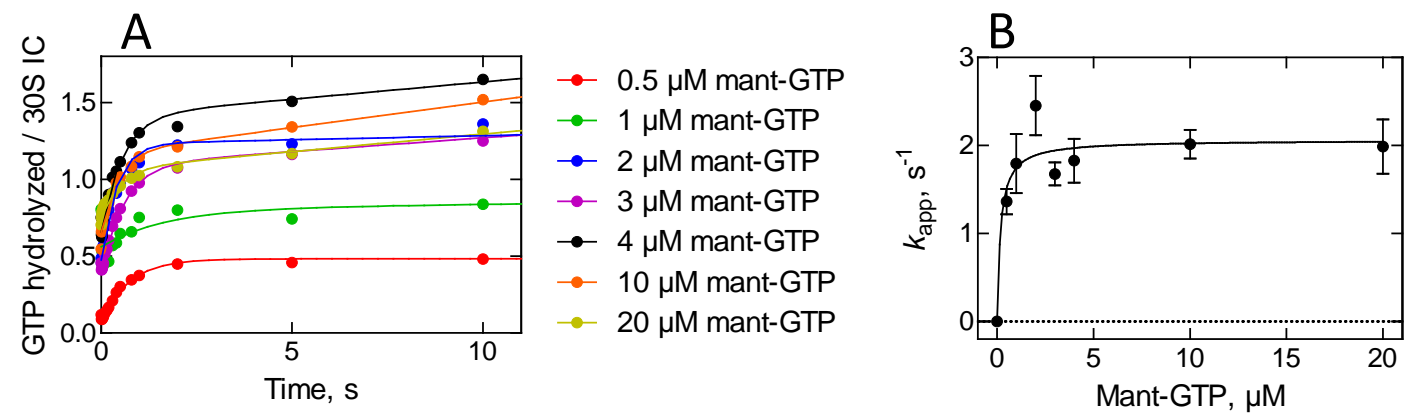

Figure 3.9 (A) The apparent rate constants of GTP hydrolysis by IF2 using different concentrations of the substrate. (B) The concentration dependence of the apparent rate constants. The $30 \mathrm{~S} \mathrm{IC}$ was formed using the following final concentrations of the components: $0.2 \mu \mathrm{M}$ 30S subunits; $0.4 \mu \mathrm{M}$ IF1; $0.25 \mu \mathrm{M}$ IF2; $0.4 \mu \mathrm{M}$ IF3; $0.4 \mu \mathrm{M}$ fMet-tRNA ${ }^{\text {fMet }}$; $0.8 \mu \mathrm{M}$ mRNA and varying concentrations of mant-GTP. The GTPase reaction was triggered by rapid mixing with $0.5 \mu \mathrm{M} 50 \mathrm{~S}$ subunits together with the respective mant-GTP concentration.

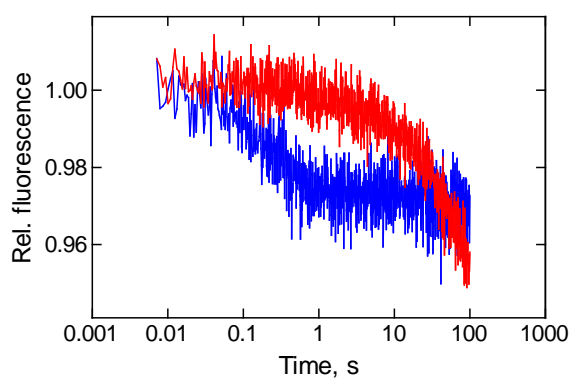

Figure 3.10 Dissociation of mant-GTP from free IF2 (blue trace) or $30 \mathrm{~S} \mathrm{IC} \mathrm{(red} \mathrm{trace).} \mathrm{30S} \mathrm{IC} \mathrm{mix} \mathrm{contained}$ (30S IC): $0.3 \mu \mathrm{M}$ 30S, $0.6 \mu \mathrm{M}$ IF1, $0.3 \mu \mathrm{M}$ IF2, $0.6 \mu \mathrm{M}$ IF3, $0.9 \mu \mathrm{M}$ mRNA, $0.6 \mu \mathrm{M}$ fMet-tRNA ${ }^{\text {fMet }}, 0.2 \mu \mathrm{M}$ mantGTP; free IF2 mix contained $0.3 \mu \mathrm{M}$ IF2 and $0.2 \mu \mathrm{M}$ mant-GTP. The dissociation of mant-GTP was started by rapid mixing with $250 \mathrm{uM}$ non-labeled GTP.

\section{Comparison of our measurements of GTP hydrolysis by IF2 to earlier studies.}

Previous studies have shown that the binding of the 50S subunits to the $30 \mathrm{~S} \mathrm{IC} \mathrm{triggers}$ fast GTP hydrolysis by IF2 which proceeds at a rate of about $30 \mathrm{~s}^{-1}$ (Grigoriadou et al., 2007a; Tomsic et al., 2000). At that time, the rates of other reactions involved in 70S IC formation, such as the $50 \mathrm{~S}$ subunit association, and dissociation of the initiation factors, was not measured due to the lack of appropriate experimental tools. Recently new 
methods have been established in our lab which allowed for measuring the rates of the reactions involved in 70S IC formation using fluorescence change and FRET. In other research groups the rates of initiation factors dissociation during the formation of 70S IC were measured using direct single molecule tracking (Tsai et al., 2012). The $50 \mathrm{~S}$ subunit was shown to join 30S IC with an association rate constant of about $10 \mu^{-1} \mathrm{~s}^{-1}$ (Milon et al., 2008) and in the living cell the rate of subunit association is expected to be ca. $10 \mathrm{~s}^{-1}$. It appears that the previously determined rate of GTP hydrolysis $\left(30 s^{-1}\right)$ is faster than the estimated rate of subunit association in the cell. In our experiments however the GTPase rate measured was about $4 \mathrm{~s}^{-1}$, which is comparable to the rates of other reactions during 70 S IC formation. Moreover we were able to fit the GTPase reaction into the scheme of 70S IC formation that was built in our lab.

There may be several reasons why the GTPase rate measured by Tomsic et al. and Grigoriadou et al. (Grigoriadou et al., 2007a; Tomsic et al., 2000) differs from that measured in the present work. We performed a number of tests concerning different conditions of the GTPase reaction and based on the results we suggest the following explanations for the observed discrepancy.

In some cases the GTPase reaction of IF2 can be triggered by the 50 S subunit alone (Fig. 3.11) (Grigoriadou et al., 2007a). However this phenomenon was observed only when particular preparations of IF2 and/or ribosome were used in the experiment and the result was not reproducible. The rate of GTP hydrolysis by IF2 triggered by 50S subunit measured by us (Fig. 3.11) is similar to the rates measured by Tomsic et al., 2000, and Grigoriadou et al., 2007, and was about $25 \mathrm{~s}^{-1}$. When using different IF2 and ribosomal preparation we did not observe GTP hydrolysis by IF2 in the presence of 50 S subunit. The rate of IF2 GTPase in the complete 30S IC in this case was $2.2 \pm 0.5$ at $20^{\circ} \mathrm{C}$ and 14.2 \pm 0.04 at $37^{\circ} \mathrm{C}$. It cannot be excluded that some preparations of IF2 had an increased propensity for GTP hydrolysis or were contaminated with other GTPase.

In cases when we observed GTP hydrolysis by IF2 without 30S IC triggered by 50S subunit, we did not find dependence of the GTPase rate from the concentration of the 50 S subunit (Fig. 3.12). This means that either IF2 is fully bound to the 50 S subunits at given concentrations or that this IF2 preparation was capable of hydrolyzing GTP without $50 \mathrm{~S}$ subunit. The rate of GTP hydrolysis in this case was in the range of $20-30 \mathrm{~s}^{-1}$, consistent with previous data (Tomsic, 2000). 


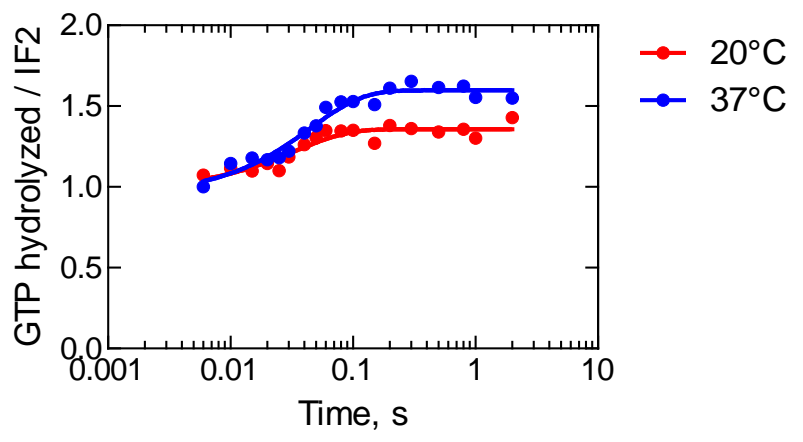

Figure 3.11 GTP hydrolysis by IF2 triggered by $50 \mathrm{~S}$ in the absence of other components of the $30 \mathrm{~S} \mathrm{IC} \mathrm{at} 20^{\circ} \mathrm{C}$ and $37^{\circ} \mathrm{C}$. The final concentrations were as follows: $0.7 \mu \mathrm{M}$ IF2; $18 \mu \mathrm{M}$ mant-GTP; $1.2 \mu \mathrm{M}$ 50S.
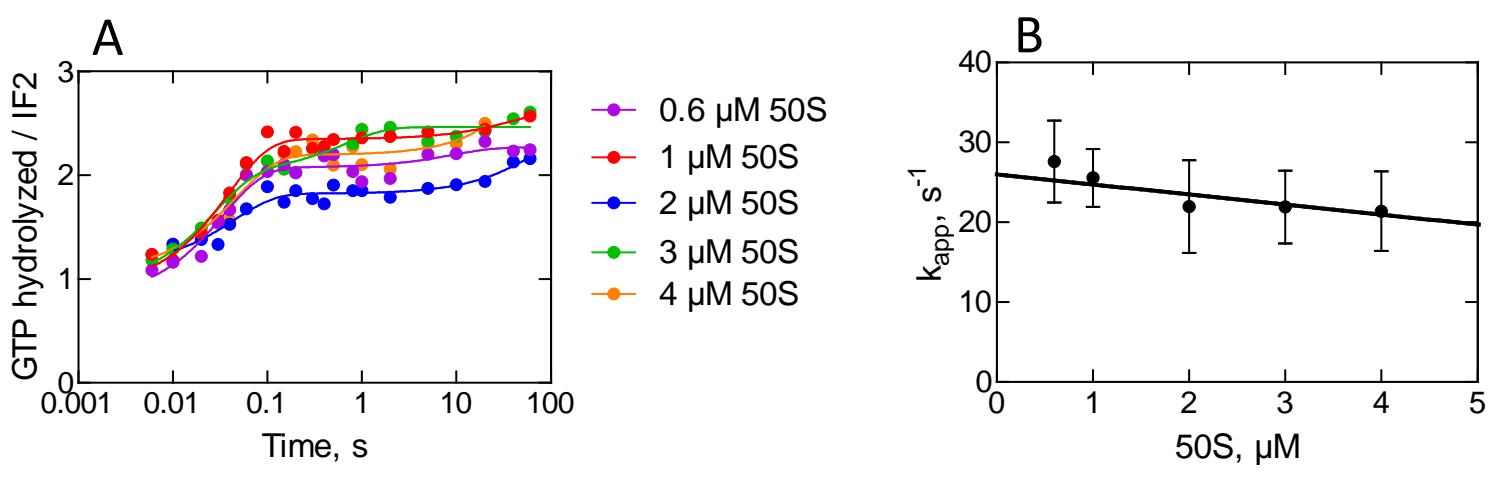

Figure 3.12 GTP hydrolysis by IF2 with increasing 50 S concentrations. (A) Time courses of reaction. (B) Apparent rate constants from A plotted against 50S concentration. The concentrations used in the reaction are as follows: $0.5 \mu \mathrm{M}$ IF2; $20 \mu \mathrm{M}$ mant-GTP; $0.3 \mu \mathrm{M}, 0.5$ $\mu \mathrm{M}, 1 \mu \mathrm{M}, 1.5 \mu \mathrm{M}, 2 \mu \mathrm{M}$ 50S.

We also measured 50S subunit-induced GTP hydrolysis by IF2, independent of addition of 30 s subunits, at increasing GTP concentrations. In all of the cases, but one (Fig. 3.13), when 30S independent IF2 GTPase was observed, the amplitude of the burst phase of GTP hydrolysis was relatively small (Fig 3.13). This reflects the portion of IF2 which is able to perform GTP hydrolysis without being bound to the 30S IC. The amplitudes of the fast GTP hydrolysis phase increased linearly with increasing GTP concentration (fig 3.13). The apparent rates of GTP hydrolysis also increased slightly with increasing GTP concentration but the dependence was not prominent (fig. 3.13). The apparent rates in this case were in the range of $25-35 s^{-1}$.

After testing different IF2 preparations for the activity of GTP hydrolysis triggered by $50 S$, we tested the same IF2 preparations for the intrinsic GTPase activity in the absence of 50 S subunits or 30 S ICs (fig 3.14). Different concentrations of IF2 were incubated with 
$1 \mu \mathrm{M}$ mant-GTP at $20^{\circ} \mathrm{C}$ for $2 \mathrm{~min}$. The reaction products were analyzed on the HPLC. Four different preparations of IF2 were tested. Astonishingly we found out that one of the IF2 preparations was able to hydrolyze GTP without the presence of the ribosome. The rest of the IF2 preparations did not hydrolyze GTP without the GTPase activator added. We explain this phenomenon by possible variations in the IF2 purification procedure which may lead to the increased capacity for the GTPase activation or by contamination of the IF2 preparation with another GTPase of similar size. For future work we took IF2 preparations which showed no intrinsic GTPase activity.
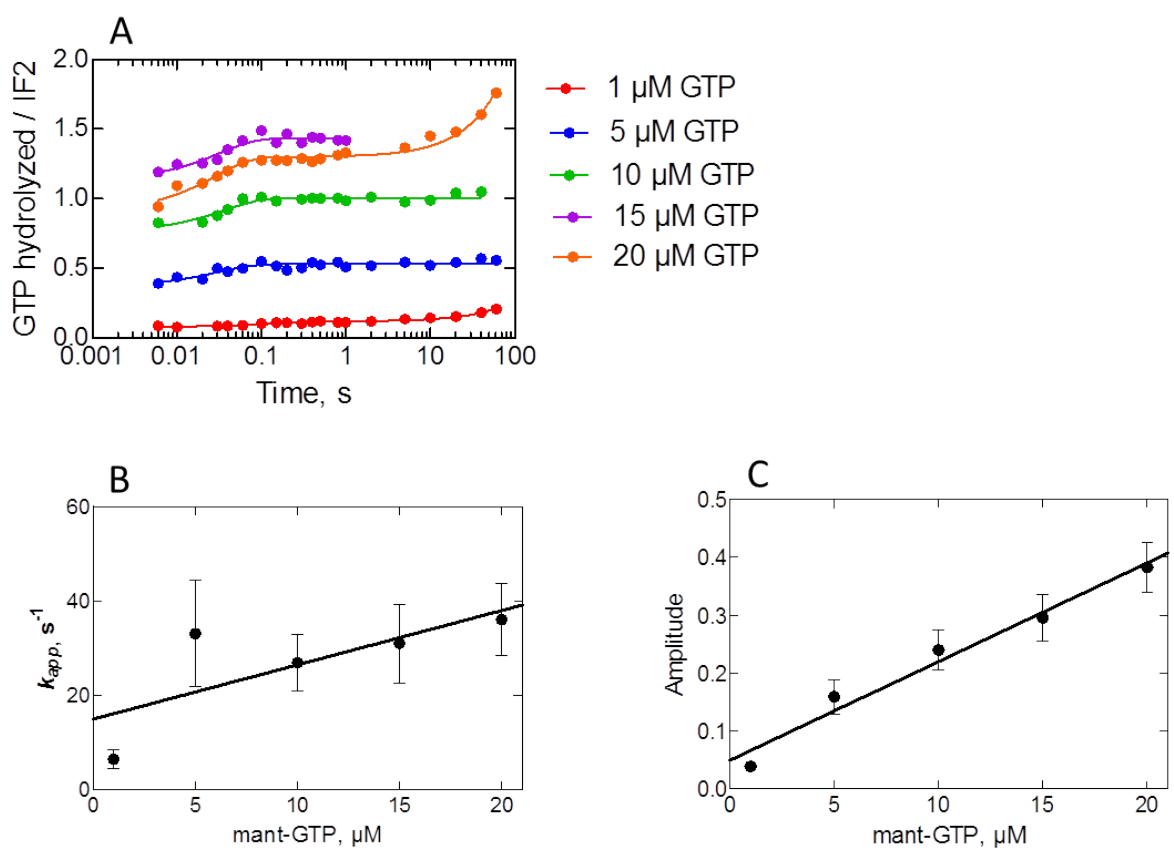

Figure 3.13 (A) GTP hydrolysis by IF2 with increasing mant-GTP concentrations. (B) Apparent rate constants from A plotted against mant-GTP concentration. (C) Amplitudes of the burst phase of the $30 \mathrm{~S}$ independent IF2 GTPase plotted against mant-GTP concentration. The final concentrations used in the reaction are as follows: $0.2 \mu \mathrm{M}$ IF2; $0.5 \mu \mathrm{M} 50 \mathrm{~S} ; 1 \mu \mathrm{M}, 5 \mu \mathrm{M}$, $10 \mu \mathrm{M}, 15 \mu \mathrm{M}, 20 \mu \mathrm{M}$ mant-GTP.

One more reason why the rate of the IF2 GTPase measured by Tomsic et al. and Grigoriadou et al. differs from the rate measured in the current work could be the different assay used for measuring the rate of GTPase reaction. In the experiments carried out by Tomsic et al. and Grigoriadou et al. the GTPase reaction was stopped by addition of the mixture of the $\mathrm{HClO}_{4}$ and $\mathrm{KH}_{2} \mathrm{PO}_{4}$ and the amount of GTP hydrolyzed was determined by molybdate extraction of $\left[{ }^{32} \mathrm{P}\right]$ phosphate. The amount of GTP hydrolyzed is quantified indirectly based on the amount of free $\left[{ }^{32} \mathrm{P}\right]$ phosphate present in the 
reaction. Instead, we used fluorescent mant-GTP in most of our experiments, the GTPase reaction was stopped by $50 \%$ formic acid and the reaction products were separated on the HPLC. The amount of GTP hydrolyzed is quantified as a ratio between the reaction products as we are able to quantify the amount of both - GTP and GDP present in the mixture before and after GTP hydrolysis have occurred.

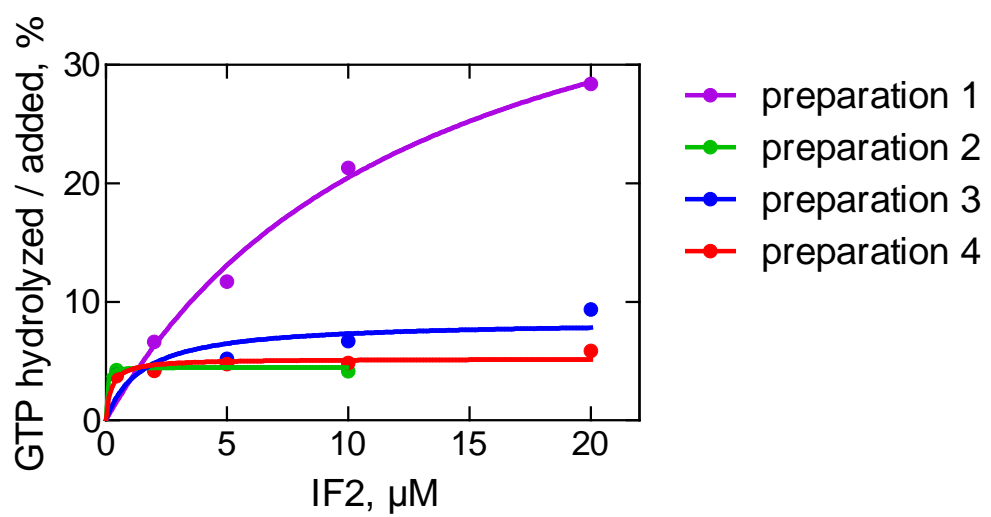

Figure 3.14 Intrinsic GTP hydrolysis by different preparations of IF2. The following concentrations were used in the reaction: $1 \mu \mathrm{M}$ mant-GTP; $0.45 \mu \mathrm{M}, 2 \mu \mathrm{M}, 5 \mu \mathrm{M}, 10 \mu \mathrm{M}$, $20 \mu \mathrm{M}$ IF2.

To exclude the possibility that mant-GTP is hydrolyzed by IF2 with a different rate than $\left[{ }^{32} \mathrm{P}\right] \mathrm{GTP}$, we performed a number of experiments using $\left[{ }^{32} \mathrm{P}\right] \mathrm{GTP}$ and mant-GTP (Fig. 3.15). The reaction products from the same mix were analyzed using TLC and HPLC. We compared multiple turnover GTP hydrolysis by IF2 in the complete 70S IC, containing all components of the complex; in the presence of 70S ribosome or in the presence of the $50 \mathrm{~S}$ subunit alone (Fig. 3.15) in the time range of $20-600 \mathrm{~s}$. We observe no significant differences in the amount of GTP hydrolyzed depending on whether the fluorescent or radioactive GTP analog was used in the reaction. Also the amount of GTP hydrolyzed did not depend on the method of separation of the reaction products (HPLC or TLC) and sample handling. As expected, the amount of GTP hydrolyzed varied considerably depending on whether the complete initiation complex was formed, involving all 3 initiation factors, fMet-tRNA ${ }^{\text {fMet }}$ and $\mathrm{mRNA}$, or only 70S ribosome and IF2 were present. In case when only IF2 and 50S subunit were present in the reaction mix, the amount of GTP hydrolyzed in 10 min was negligible (less than 5\%). In case when GTP hydrolysis was measured in the presence of $70 \mathrm{~S}$ alone, about $25 \%$ of GTP was hydrolyzed, which corresponds to the background level of GTP hydrolysis observed in the previous experiment where fMet-tRNA ${ }^{\text {fMet }}$ was omitted from the system (Fig 3.8). 


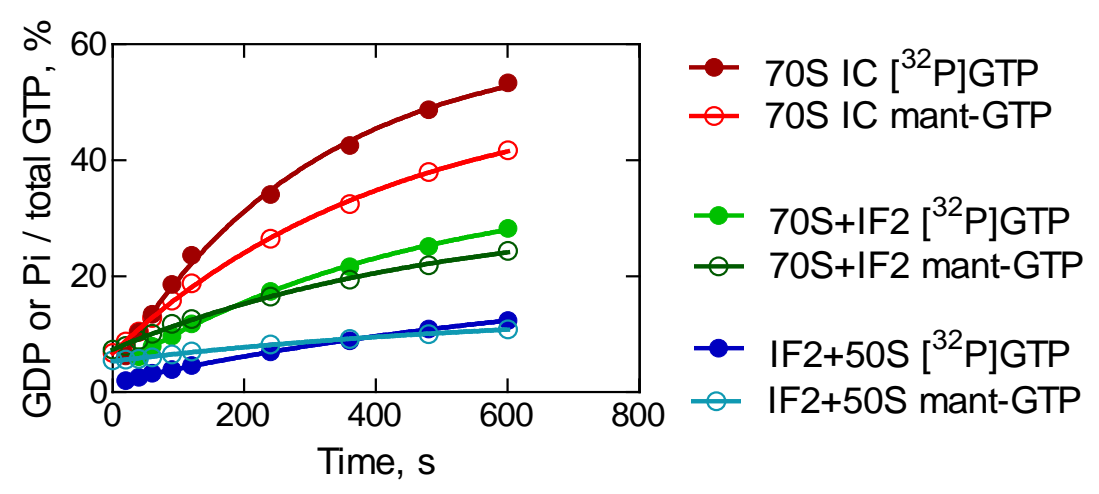

Figure 3.15 Comparison of mant-GTP and $\left[{ }^{32} \mathrm{P}\right] \mathrm{GTP}$ hydrolysis by IF2 in the presence of complete 70S IC (red curves), 70S ribosome alone (green curves) and $50 \mathrm{~S}$ subunit alone (blue curves). The following concentrations of the components were used: 1) complete $70 \mathrm{~S}$ IC $-0.4 \mu \mathrm{M}$ 30S, $1 \mu \mathrm{M}$ IF1, $0.9 \mu \mathrm{M}$ IF2, $1 \mu \mathrm{M}$ IF3, $0.8 \mu \mathrm{M}$ fMet-tRNA ${ }^{\text {fMet }}, 1.2 \mu \mathrm{M}$ mRNA, 36 $\mu \mathrm{M}$ mant-GTP/ $\left.\left[{ }^{32} \mathrm{P}\right] \mathrm{GTP} ; 2\right) 70 \mathrm{~S}$ alone - $0.4 \mu \mathrm{M}$ 30S, $0.9 \mu \mathrm{M}$ IF2, $36 \mu \mathrm{M}$ mant-GTP/ $\left[{ }^{32} \mathrm{P}\right] \mathrm{GTP}$; 3) IF2 alone $-0.9 \mu \mathrm{M}$ IF2, $36 \mu \mathrm{M}$ mant-GTP/[32 P] GTP. All the reactions were triggered by addition of $2 \mu \mathrm{M} 50 \mathrm{~S}$.

We also checked for the rapid phase of GTP hydrolysis by IF2 triggered by 50 S subunit alone using mant-GTP and $\left[{ }^{32} \mathrm{P}\right]$ GTP in the same reaction mix. GTP hydrolysis in the time range of $0.005-20 \mathrm{~s}$ was measured. The reaction products were separated on TLC and HPLC (Fig. 3.16). However, with these particular preparations of IF2 and 50 S no rapid GTP hydrolysis was observed. As expected, there was some slow background GTP hydrolysis which increases linearly with time. The reaction showed no dependence on whether mant-GTP or $\left[{ }^{32} \mathrm{P}\right] \mathrm{GTP}$ was used.

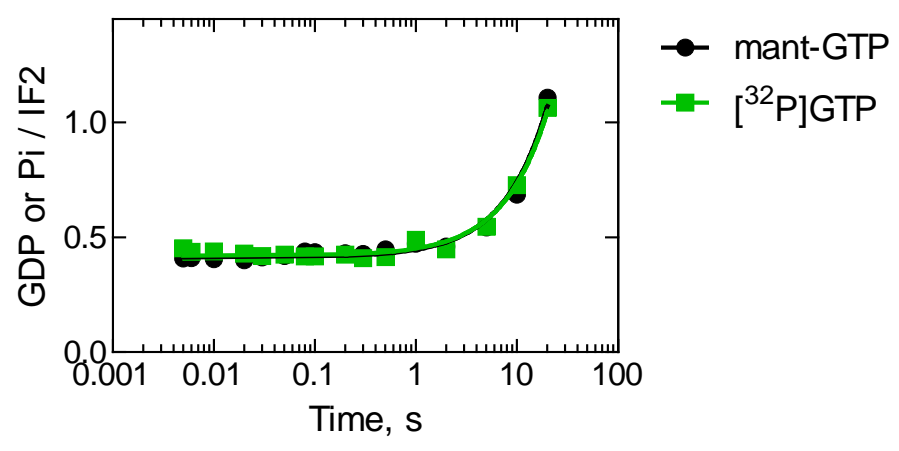

Figure 3.16 GTP hydrolysis by IF2 triggered by $50 \mathrm{~S}$ subunit alone. No fast phase of GTP hydrolysis is observed using these preparations of IF2 and 50S. The data obtained with mant-GTP or [ $\left.{ }^{32} \mathrm{P}\right] \mathrm{GTP}$ in the reaction are exactly the same. The concentrations of the components are as follows: $2 \mu \mathrm{M}$ IF2; $20 \mu \mathrm{M}$ mix of mant-GTP and $\left[{ }^{32} \mathrm{P}\right] \mathrm{GTP} ; 4 \mu \mathrm{M} 50 \mathrm{~S}$. 
The last reason for the different rates of IF2-catalyzed GTPase reaction observed in the presented work and in the work of Grigoriadou et al. could be that IF2 from different organisms were used. Grigoriadou et al. used IF2 purified from B. stearothermophilus which is a Gram-positive thermophile, whereas we used IF2 purified from E. coli which is a Gram-negative mesophile. It was reported that IF2 from B. stearothermophilus is able to hydrolyze GTP in the absence of ribosomes which creates the additional GDP background (Severini et al., 1991). This however does not explain why the rate of IF2 GTPase measured in the work of Tomsic et al. corresponds to the rate of Grigoriadou et al. but not measured by us, as they used IF2 purified from E. coli as well.

\section{Discussion.}

\section{Validation of the GTPase assay.}

Kinetic studies of GTPase reactions require sensitive methods which allow for precise quantification of the amounts of GTP hydrolyzed. The reaction has to be stopped rapidly and the products separated and quantified. In single turnover GTPase reaction the amount of hydrolyzed GTP is low; therefore, it is not possible to detect the reaction products via absorption measurements. Previously $\left[\mathrm{r}^{-32} \mathrm{P}\right] \mathrm{GTP}$ or $\left[{ }^{3} \mathrm{H}\right] \mathrm{GTP}$ was used in the most studies of the GTPase reactions. The methods for quantifying the reaction products were the following: extraction of radioactive $\mathrm{Pi}$ into the dodecamolybdate complex or analysis of GTP and GDP ratio by thin layer chromatography (TLC). Both methods have a disadvantage of having high radioactive background and long hand-on times. One more disadvantage of working with $\left[\gamma^{-32} \mathrm{P}\right]$ GTP-based assay is that the stock solutions of the $\left[\mathrm{Y}^{-}{ }^{32} \mathrm{P}\right] \mathrm{GTP}$ can contain radioactive contaminations which increase the radioactive background in the sample therefore leading imprecise quantification. We developed an alternative HPLC-based method for separation of the substrates/products of the GTPase reaction. The procedure can utilize fluorescent or radioactive (tritium-labeled) nucleotide derivatives. When using GTP derivatives labeled in the guanine-base ring, it is possible to quantify both - the remaining substrate (GTP) after the reaction and the reaction product (GDP). Calculating the ratio product/substrate, rather than absolute amounts, eliminates the possible error due to the sample handling.

We performed a number of experiments where we compared GTP hydrolysis by IF2 using mant-GTP, $\left[{ }^{3} \mathrm{H}\right]$ GTP and/or $\left[{ }^{32} \mathrm{P}\right] \mathrm{GTP}$ in parallel. However, the use of fluorescent GTP derivatives is possible only when the attached fluorophore does not alter the 
affinity of GTP to the GTPase, which was shown for the other prokaryotic and eukaryotic translational GTPases (Pisareva et al., 2006, 2007; Savelsbergh et al., 2000; Wilden et al., 2006). If the fluorescent group interferes with the GTPase function, it is recommended to use $\left[{ }^{3} \mathrm{H}\right]$ GTP. The established assay is suitable for measuring both single turnover and multiple turnover reactions of GTP and ATP hydrolysis. In our group it was successfully used for measuring the rapid kinetics of GTP hydrolysis by IF2 and EF-G.

\section{The rate of GTP hydrolysis by IF2 compared to the previous works.}

The rate of the IF2-catalyzed GTP hydrolysis measured in this work appeared to be an order of magnitude lower than the rate of GTP hydrolysis measured in previous works (Grigoriadou et al., 2007a; Tomsic et al., 2000) which was around $30 \mathrm{~s}^{-1}$. However, the rate of GTP hydrolysis measured by is in line with the rates of other reactions during formation of the 70S IC measured in our lab (Milon et al., 2008). We can suggest several reasons for the differences in the IF2 GTPase rates.

Firstly, it is possible that GTPase activity of IF2 can be triggered by isolated 50 S subunit. Such hydrolysis in the absence of the other components of the 30S IC can happen at a rate much higher than the rate of GTP hydrolysis by IF2 in the presence of 30 S subunit $\left(30 \mathrm{~s}^{-1}\right.$ vs $\left.4 \mathrm{~s}^{-1}\right)$. Taking into consideration that the affinity of GTP to the 30S-bound IF2 is higher than to the free IF2, it is expected that at cellular GTP concentrations most of it will be bound to IF2 on the 30S IC. As Tomsic et al. carried out the GTPase reaction in the presence of large excess of GTP $\left(0.6 \mu \mathrm{M}\right.$ 30S, $0.9 \mu \mathrm{M}$ IF2, $72 \mu \mathrm{M}\left[{ }^{32} \mathrm{P}\right] \mathrm{GTP}, 0.9 \mu \mathrm{M}$ IF1, 0.9 $\mu \mathrm{M}$ IF3, $0.9 \mu \mathrm{M}$ fMet-tRNA ${ }^{\mathrm{fMet}}, 1.8 \mu \mathrm{M}$ mRNA; reaction triggered by $0.6 \mu \mathrm{M}, 1.2 \mu \mathrm{M}$ or $1.6 \mu \mathrm{M} 50 \mathrm{~S}$ ), it was possible that a portion of GTP was hydrolyzed by free IF2 triggered by free 50S. Secondly, we have observed that one of our IF2 preparations was able to perform GTP hydrolysis in solution in the absence of any activator possibly due to the contamination of this preparation by some other GTPase. Last, Grigoriadou et al. used IF2 purified form B. stearothermophilus, whereas in our study we used IF2 purified from E. coli. We cannot exclude that IF2 from different organisms can hydrolyze GTP with different rates at similar conditions.

At cellular concentrations of the initiation components the rate of $50 \mathrm{~S}$ subunit joining is expected to be about $10 \mathrm{~s}^{-1}$. The rates of IF1 and IF3 dissociation are about $3 \mathrm{~s}^{-1}$ (Milon et al., 2008). The rate of IF2 dissociation is $1.4 \mathrm{~s}^{-1}$ (Milon et al., unpublished data) which coincides with the previously measured rate of Pi release (Tomsic et al., 2000). The rate 
of GTP hydrolysis measured by us in the present work (about $4 \mathrm{~s}^{-1}$ ) fits into this kinetic scheme of 70S IC formation events.

\section{Kinetics and role of GTP hydrolysis by IF2.}

GTP hydrolysis by IF2 is an important step of initiation. GTPase-deficient mutants of $E$. coli were either not viable, or the growth of the cells was impaired (Laalami et al., 1994; Luchin et al., 1999). IF2 in complex with GTP (or non-hydrolysable GTP analog) bound to the 30 S IC was shown to promote rapid 50S subunit association resulting in 70S IC formation (Antoun et al., 2003; Grigoriadou et al., 2007a). On the contrary, if IF2 in complex with GDP was present on the 30S IC, 50 S association was 50 times slower (Antoun et al., 2003). No dipeptide formation was observed in the absence of GTP hydrolysis by IF2 when GTP was replaced by the non-hydrolysable analogs (Antoun et al., 2003). Also IF2 dissociation from the ribosome was impaired when either nonhydrolysable GTP analog was used (Antoun et al., 2003) or GTPase deficient mutant (IF2 H448S) was present in the reaction (Laalami et al., 1994; Luchin et al., 1999).

Interesting findings concerning the role of GTP hydrolysis by IF2 were presented in the work of Fabbretti et al. (Fabbretti et al., 2012). Two GTPase deficient mutants of IF2 were studied - IF2 E571K (E. coli) and IF2 E424K (B. stearothermophilus). Unlike the GTPase deficient mutant IF2 H448S (Laalami et al., 1994; Luchin et al., 1999), the mutation in both IF2 mutants E571K and E424K was not in the active center, but in the G3 domain. This mutation is thought to alter the conformation of IF2 disrupting the communication between domains $G 2$ and G3. The change in the structure most likely affects the position of catalytic H448 (E. coli) and H301 (B. stearothermophilus) which leads to GTPase inactivation (Voorhees et al., 2010). The mutant factors (IF2 E571K and E424K) are possibly locked in the GDP-bound conformation. Although both mutants are able to bind both, GTP and GDP, the affinity for the GTP is decreased 4-fold compared to wild type (wt) IF2, whereas affinity to GDP is increased 6-fold in comparison to wt IF2. Astonishingly, despite the absence of GTPase function, IF2 E571K was shown to support in vitro $30 \mathrm{~S} \mathrm{IC} \mathrm{formation,} \mathrm{the} 50 \mathrm{~S}$ subunit association with the $30 \mathrm{~S} \mathrm{IC,} \mathrm{dipeptide}$ formation and mRNA translation, although to a lesser extent than wt IF2. The reduced activity of the factor is attributed not to the lack of GTPase, but rather to its GDP-bound conformation. Moreover, the IF2 E424K from B. stearothermophilus could substitute for IF2 in the E. coli IF2 null strain allowing cells to grow with almost wt duplication time. The study shows the importance of the GTP-bound IF2 conformation for the translation 
initiation steps occurring before GTP hydrolysis, namely interaction of IF2 with the $30 \mathrm{~S}$ subunit, recruitment and stabilization of the fMet-tRNA ${ }^{\text {fMet }}$ on the ribosome and docking of the $50 \mathrm{~S}$ subunit. However the free energy generated by the IF2-catalyzed GTP hydrolysis is not required for progression of the ribosome to the elongation step. The mRNA was shown to be translated in vitro and in vivo without GTPase function of IF2 (Fabbretti et al., 2012)

GTP hydrolysis by IF2 is triggered by the 50S subunit association with the $30 \mathrm{~S} \mathrm{IC}$ (Grigoriadou et al., 2007a, 2007b; Tomsic et al., 2000). The observed rate of GTP hydrolysis in our experiments was about $2 \mathrm{~s}^{-1}$. Evaluation of the time courses of GTP hydrolysis by numerical integration yielded the rate constant of $3.7 \mathrm{~s}^{-1}$. We found that the rate of GTP hydrolysis by IF2 did not depend on the concentration of the substrate, supporting the previous observation (Antoun et al., 2003; Pon et al., 1985) that affinity of IF2 to GTP differs depending on whether IF2 is free or ribosome-bound. The rate of IF2 GTPase did not depend on the concentration of the 50S subunits, although the rate of subunits association increases linearly with the increasing of $50 \mathrm{~S}$ concentration (Milon et al., 2008). Neither did the rate of IF2 GTPase depend on the presence of IF1 and/or IF3 in the 30S IC. It was previously observed in our lab (Milon et al., 2008) that the rate of subunits association increases 6 times in the absence of IF3. Nevertheless, omitting IF3 from the system did not affect the rate of GTP hydrolysis by IF2. This suggests structural rearrangements in the initiation complex upon association of the subunits. The structural rearrangements may involve overall ribosomal landscape change as well GTPase activation step.

We observed absolutely no GTP hydrolysis by IF2 when fMet-tRNA ${ }^{\mathrm{fMet}}$ was omitted form the 30S PIC. IF2 in its GTP bound form is known to form the interaction with fMettRNA $^{\text {fMet }}$ on the $P$ site of the ribosome (Allen et al., 2005; Myasnikov et al., 2005). It was also shown that affinity of GTP to IF2 is increased if fMet-tRNA ${ }^{\text {fMet }}$ is present in the $30 \mathrm{~S}$ IC (Antoun et al., 2003). It is possible that without the contact with fMet-tRNA ${ }^{\text {fMet }}$ on the $30 S$ IC, IF2 is in a GTPase inactive conformation or in the wrong orientation so that $50 \mathrm{~S}$ subunit is not able to trigger IF2 GTPase activity.

In summary, we conclude that during the 70S IC formation, IF2 functions independently from IF1 or IF3. The fate of IF2 as a part of initiation complex can be described as follows. IF2 binds the $30 \mathrm{~S} \mathrm{IC} \mathrm{in} \mathrm{the} \mathrm{GTP-bound} \mathrm{form.} \mathrm{Association} \mathrm{of} \mathrm{the} 50 \mathrm{~S}$ subunit triggers IF2-catalyzed GTP hydrolysis if the essential elements of the 70S (fMet-tRMA ${ }^{\text {fMet, }}$, mRNA) IC are present. As the rate of GTP hydrolysis does not depend on either 
substrate, or GTPase activator concentration, one can suggest that there are some conformational rearrangements in the entire complex at this step involving the GTPase activation. After GTP hydrolysis occurred, IF2 undergoes a conformational change (Allen et al., 2005; Myasnikov et al., 2005) accepting "ready-to leave" conformation and disrupting the interaction with $\mathrm{fMet-tRNA} \mathrm{fMet}^{\mathrm{P}}$. The latter becomes positioned in the $\mathrm{P}$ site of the ribosome. Subsequently, hydrolyzed phosphate group is released from the ribosome (Tomsic et al., 2000) as well as the factor itself. Perhaps IF2 can be called a landscape sensor as only in the properly formed 70S IC IF2 GTPase is triggered, which leads further to the Pi and IF2 dissociation from the complex, rotation of the ribosomal subunit from the "rotated" to the "non-rotated" state (Marshall et al., 2009) and progression of the whole system to the elongation (Grigoriadou et al., 2007a; Tomsic et al., 2000). 


\section{Chapter 4. Reconstitution, Functional Analysis and Structural Studies of the Translation Initiation Complex of T. thermophilus.}

\section{Introduction.}

The structures of the 30S IC and 70S IC were solved using the cryo-electron microscopy (Allen et al., 2005; Julián et al., 2011; Myasnikov et al., 2005; Simonetti et al., 2008). In these works the location of initiation factors, $f M e t-t R N A^{\text {fMet }}$ and $m R N A$ on the ribosome were shown. Comparison of these structures suggests which conformational changes occur in the complex upon the transition from the 30S IC to the 70S IC. However, up to date there is no crystal structure of the translation initiation complex available. In the current work we aimed to crystallize 70S IC from $T$. thermophilus containing fMettRNA ${ }^{\mathrm{fMet}}$, mRNA and IF2 in complex with guanine nucleotides.

The conformation of the $30 \mathrm{~S}$ subunit was found to be different depending on the presence of the initiation factors in the complex (Julián et al., 2011). The vacant 30S exists in the so-called non-rotated state (Julián et al., 2011). In the presence of the initiation factors, FMet-tRNA $^{\text {fMet }}$ and mRNA the head of the $30 \mathrm{~S}$ subunit is rotated with respect to the body, which is called the rotated state (Julián et al., 2011). The same conformation of the $30 \mathrm{~S}$ subunit is observed after the $50 \mathrm{~S}$ subunit docking but prior to GTP hydrolysis by IF2 (Allen et al., 2005). After GTP hydrolysis by IF2, the ribosome changes conformation to the non-rotated state (Marshall et al., 2009; Myasnikov et al., 2005).

The dimerization surface of the $30 \mathrm{~S}$ and $50 \mathrm{~S}$ is composed of negatively charged rRNA (Allen et al., 2005) which creates the electrostatic barrier for the subunit joining. Upon subunit joining twelve intersubunit bridges are formed which are comprised of RNA-RNA interactions. On the vacant ribosome, $\mathrm{Mg}^{2+}$ ions help the formation of the bridges partially compensating for the electrostatic barrier (Schuwirth et al., 2005; Yusupov et al., 2001). IF2 was located at the intersubunit cleft of the 705 ribosome (Allen et al., 2005; Julián et al., 2011; Myasnikov et al., 2005). The ribosome-bound IF2 facilitates 50S subunit joining partially masking the negative charge of the ribosome and providing the large surface area for 50 S subunit docking (Julián et al., 2011; Marshall et al., 2009). After IF2 performs GTP hydrolysis, there are conformational rearrangements in the G1, G2 and C1 domains of the factor (corresponding to the G-domain and domains II and III of the alF5B) (Roll-Mecak et al., 2000). Consequently, the intersubunit bridges are 
exposed (Myasnikov et al., 2005), which promotes 50S association and the subunits rotation (Marshall et al., 2009).

The structural information about bacterial IF2 is based on the biochemical experiments (Gualerzi et al., 1991) and homology models (Allen et al., 2005; Julián et al., 2011; Myasnikov et al., 2005; Roll-Mecak et al., 2000; Simonetti et al., 2009).The structure of separate IF2 domains was solved by NMR (Laursen et al., 2003; Meunier et al., 2000; Wienk et al., 2005, 2012) and SAXS (Rasmussen and Oliveira, 2008). However, being bound to the ribosome, IF2 adopts the conformation totally different from that of its crystallized archaeal homolog alF2 (Allen et al., 2005). IF2 was shown to have two anchor points on the $30 \mathrm{~S}$ subunit: one through its NTD forming an interaction with the ribosomal protein S12 and one through the CTD touching the acceptor end of the fMettRNA $^{\text {fMet }}$ (Julián et al., 2011; Simonetti et al., 2008) (Fig 4.1, Fig 4.4 A). The direct interaction of IF2 with the fMet-tRNA ${ }^{\text {fMet }}$ was observed (Julián et al., 2011) (Fig 4.1).

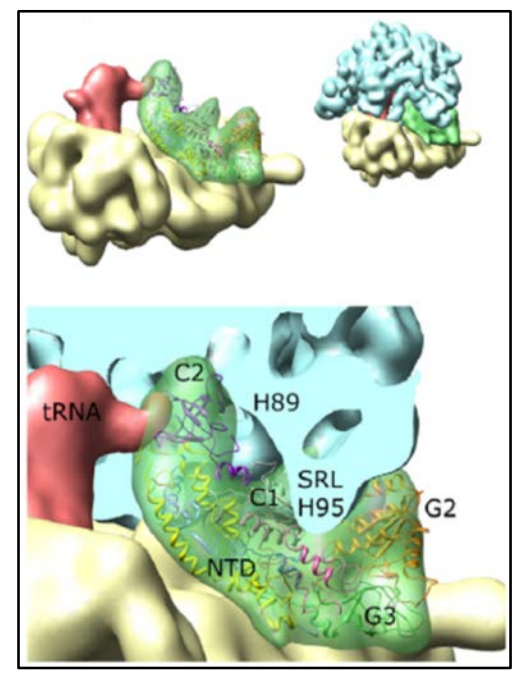

Figure 4.1 (from Julian et al., 2011) The position of IF2 (green) and fMet-tRNA ${ }^{\text {fMet }}$ (red) on the 30 S subunit (yellow). The $50 \mathrm{~S}$ subunit (blue) position is modeled from the alignment with the cryo-EM reconstruction of the 70S IC (Allen et al., 2005). The position of the sarcin-ricin loop (SRL) at the cleft formed by domains $\mathrm{G} 2$ and $\mathrm{C} 1$ of IF2 is indicated. Labels on the $50 \mathrm{~S}$ subunit indicate helices in $23 \mathrm{~S}$ rRNA (H89 and H95) and the stalk region for protein L1.

The conformation of IF2 changes during the initiation process. Comparison of the structures of IF2 in the GTP-bound form before and after the 50S subunit joining revealed the existence of several distinct conformations of IF2 (Simonetti et al., 2008) (Fig 4.3). The conformation of IF2 also depends on the nucleotide bound (Myasnikov et al., 2005) (Fig 4.2). Transition from GTP-bound to GDP-bound form involves significant rearrangements in the factor as well as in the entire ribosome. The 70S IC structure with IF2 in the GTP-bound form represents the state of the complex right after the $50 \mathrm{~S}$ subunit joining, whereas the GDP-bound form of IF2 represents the state right before IF2 dissociation from the ribosome. In the GTP-bound form, IF2 interacts mostly with the 
small ribosomal subunit and with the initiator tRNA. In the GDP-bound form, the CTD of IF2 is rotated counter-clockwise along its long axis as compared to GTP-bound-form (Myasnikov et al., 2005; Simonetti et al., 2008)(Fig. 4.2, Fig 4.3).

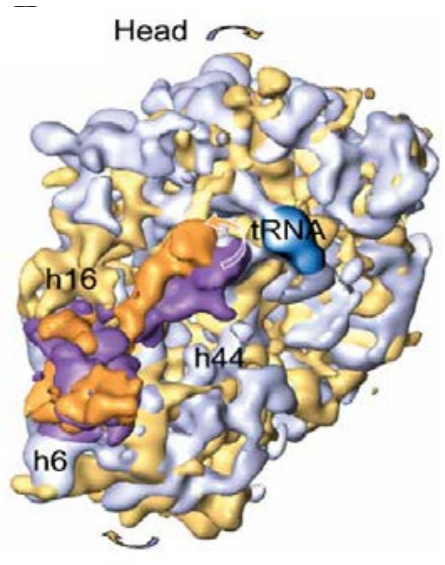

Figure 4.2 (from Myasnikov et al., 2005) Cryo-EM structure of the 70S IC form $T$. thermophilus. Only small ribosomal subunit is shown in the picture. IF2 in the GTP-bound state (purple) and GDP-bound state (orange) are superimposed on the 30S; tRNA is shown in blue. GDPCP is used to model GTP-bound state of IF2. The state of the 30S with IF2GDPCP is shown in light purple; with IF2-GDP in light orange. Upon transition from the GTP-bound state to the GDP-bound state, IF2 loses the contact with P-site tRNA, and interactions with the decoding region and the ribosomal GTPase domain are reduced. The associated conformational change of the ribosome is a slight rotation of the $30 \mathrm{~S}$ subunit with respect to the $50 \mathrm{~S}$ subunit (the head movement is shown with an arrow; helix 6, 16 and 44 are marked as h6, h16 and h44.

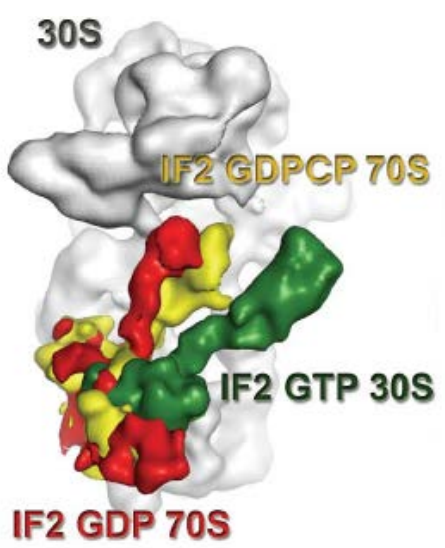

Figure 4.3 (from Simonetti et al., 2008) Positions of IF2 on the 30S subunit: green - before $50 \mathrm{~S}$ joining, yellow - after $50 \mathrm{~S}$ subunit joining but prior to GTP hydrolysis (form Myasnikov et al., 2005), red - after 50 S subunit and after GTP hydrolysis (from Myasnikov et al., 2005). The $30 \mathrm{~S}$ model is shown in gray.

Being in the GTP-bound form, CTD of IF2 contacts the single stranded acceptor stem of fMet-tRNA $^{\mathrm{fMet}}$ and the formylated methionine (Guenneugues et al., 2000; Petersen et al., 1979, 1981; Wakao et al., 1989), thus placing the initiator tRNA in the P/I position (Allen et al., 2005) (Fig. 4.4). This position was discovered only for initiator tRNA (Allen et al., 2005). It is suggested that $P / I$ position may serve as a checkpoint for discrimination against non- initiator tRNAs. It may require higher standard free energy for any tRNA in the $P / I$ site than in the classical position in the $P$ site, and the energy barrier may be too high for the elongator tRNAs (Allen et al., 2005). After GTP hydrolysis, fMet-tRNA ${ }^{\text {fMet }}$ is 
positioned into the ribosomal $\mathrm{P}$ site which is also coupled to the ribosomal subunits rotation relative to one another (Marshall et al., 2009; Myasnikov et al., 2005). The CTD

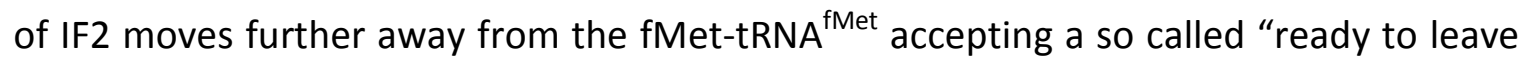
conformation" (Myasnikov et al., 2005) (Fig 4.2). As the interaction surface of IF2 with the ribosome is decreased upon these conformational rearrangements, this also stimulates the dissociation of the factor (Allen et al., 2005).

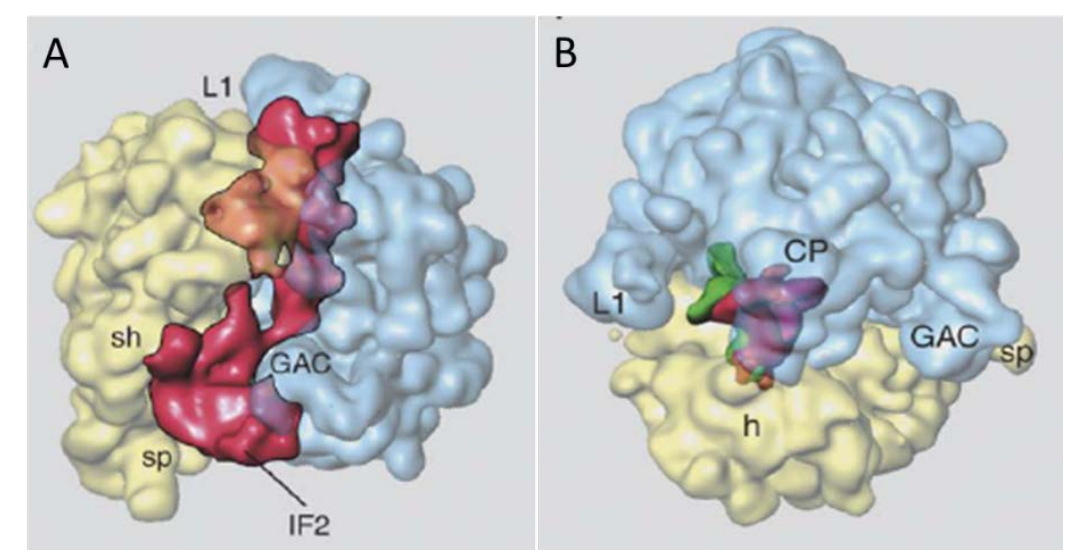

Figure 4.4 (from Allen et al., 2005) (A) Cryo-EM Structure of the 70S IC from E. coli with IF2 in complex with GDPNP. IF2 together with tRNA are shown in red with black outline. IF2 and tRNA interact with the 50 S subunit (blue) at the GTPase associated center (GAC) and with the 30 subunit (yellow) over a large surface on the intersubunit side of the shoulder (sh). Ribosomal spur (sp) and the L1 stalk (L1 are also indicated. (B) Comparison of tRNA in the P/I Site (green) of the initiation complex with its $P$ Site (red) and $P / E$ Site (purple) positions. The central protuberance is indicated as CP; h, head; sp, spur; L1, L1 stalk; GAC, GTPase center.

All translational GTPases bind to the same region on the 50S called the GTPase activating center and their binding is mutually exclusive. The elongator ternary complex binding cannot occur prior to the release of the IF2 from the ribosome. Therefore the transition of the complex from initiation to elongation may be controlled at this step (Grigoriadou et al., 2007a; Myasnikov et al., 2005). The G-domains of IF2, EF-Tu and EF-G occupy approximately the same position on the ribosome and in similar orientations, though they exhibit structural differences (Allen et al., 2005). The mechanism by which the ribosome activates GTP hydrolysis is suggested to be common for all translational GTPases (Voorhees et al., 2010) as there is no GTPase activator protein involved. In fact, there are no ribosomal proteins in the vicinity of the binding site of the IF2 or the other translational GTPases (Allen et al., 2005; Voorhees et al., 2010). Stimulation of GTP hydrolysis on the ribosome requires the positioning of a catalytic histidine of the factor 
into the active site by the sarcin-ricin loop (SRL) residue A2662. GTPase activation by the SRL is suggested to be universal and conserved in all kingdoms of life (Voorhees et al., 2010).

Lacking crystal structure of the 70S IC, a number of important questions about translation initiation remains opened: (1) how does the IF2 position on the ribosome change upon 505 joining?; (2) what rearrangements occur in the complex upon GTPase activation and GTP hydrolysis? (3) how exactly is IF2 released from the 70S IC? The current investigation aimed to answer these questions through the crystallization of the 70S IC from T. thermophilus.

\section{Materials and methods.}

mRNA (5'-GGG AAG GAG GUA AAA $\underline{\text { AUG }}$ AUC AAA AAA AAA-3') was purchased from Dharmacon.

\section{Preparations.}

70SAL9.

T. thermophilus cells for preparation of the $70 \mathrm{~S} \Delta \mathrm{L} 9$ ribosomes were grown commercially (Brown University, Rhode Island, USA) and stored at $-80^{\circ} \mathrm{C}$.

\section{Buffers.}

BufferA: 20 mM Hepes-KOH pH 7.5, $100 \mathrm{mM} \mathrm{NH}_{4} \mathrm{Cl}, 10.5 \mathrm{mM} \mathrm{MgAc}_{2}, 0.5 \mathrm{mM}$ EDTA, 1

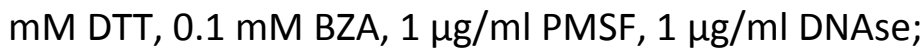

BufferB: 20 mM Hepes-KOH pH 7.5, $1.1 \mathrm{M}$ sucrose, $500 \mathrm{mM} \mathrm{KCl}, 10.5 \mathrm{mM} \mathrm{MgAc}, 1 \mathrm{mM}$ DTT;

Buffer C: 20 mM Tris- $\mathrm{HCl}$ pH 7.5, $10 \mathrm{mM} \mathrm{MgCl}, 400 \mathrm{mM} \mathrm{NaCl}, 1 \mathrm{M}(\mathrm{NH} 4)_{2} \mathrm{SO}_{4}, 0.1 \mathrm{mM}$ EDTA, 1 mM DTT;

Buffer D: 20 mM Tris- $\mathrm{HCl}$ pH 7.5, $10 \mathrm{mM} \mathrm{MgCl}$, 400 mM NaCl, 0.1 mM EDTA, 1 mM DTT; 
Buffer E 5x: 50 mM Hepes-KOH pH 7.5, $51.25 \mathrm{mM} \mathrm{MgAc}_{2}, 50 \mathrm{mM} \mathrm{NH}_{4} \mathrm{Cl}, 250 \mathrm{mM} \mathrm{KCl}$, 1.25 mM EDTA, 1 mM DTT;

Buffer G: 5 mM Hepes-KOH pH 7.5, $10 \mathrm{mM} \mathrm{MgAc}_{2}, 10 \mathrm{mM} \mathrm{NH}_{4} \mathrm{Cl}, 50 \mathrm{mM} \mathrm{KCl}, 1.25 \mathrm{mM}$ EDTA, 1 mM DTT;

Sucrose gradients buffer 1: $5 \%$ sucrose in $1 \times$ buffer $E$;

Sucrose gradient buffer 2: $20 \%$ sucrose in $1 x$ buffer $E$.

After $\mathrm{pH}$ adjustment all solutions were filtered through a $0.2 \mu \mathrm{m}$ cellulose acetate filter (Sartorius Stedim).

If required, DTT, BZA, PMSF and DNAse were added to the buffers right before use.

$30 \mathrm{~g}$ of cells were used for each ribosome preparation. The cells were thawed in Buffer $\mathrm{A}$ in a beaker at $4^{\circ} \mathrm{C}$ with constant stirring for $2 \mathrm{~h}$. The cell suspension was filtered through gauze and further diluted to $170 \mathrm{ml}$ with buffer $A$. The cells were disrupted by 3 passes through an M-110EH-30 Microfluidizer Proseccor (Microfluidics, USA) at 20000 psi. The cell lysate was centrifuged using a JLA-16.25 rotor at $12500 \mathrm{rpm}$ for $45 \mathrm{~min}$. The volume of the supernatant was then increased to $240 \mathrm{ml}$ with Buffer A. Six Ti45 tubes were filled with $25 \mathrm{ml}$ of buffer B (sucrose cushion) and $40 \mathrm{ml}$ of cell lysate were carefully overlaid on each sucrose cushion. The ribosomes were purified through the sucrose cushion by centrifugation in a Beckman Ultracentrifuge using a Ti45 rotor at $45000 \mathrm{rpm}, 4^{\circ} \mathrm{C}, 12 \mathrm{~h}$.

After centrifugation through the sucrose cushion, the supernatant was discarded, and the pellet was washed twice with $10 \mathrm{ml}$ of Buffer C. Each pellet was dissolved in $8 \mathrm{ml}$ Buffer $\mathrm{C}$ using small magnetic stirrers at $4^{\circ} \mathrm{C}$. The dissolved samples were combined and centrifuged in a JA 25.50 rotor at $10000 \mathrm{rpm}$ for $10 \mathrm{~min}$ at $4^{\circ} \mathrm{C}$. The supernatant was filtered through $0.2 \mu \mathrm{M}$ sterile filters. The final volume of the sample was $50 \mathrm{ml}$. The purification of $70 \mathrm{~S}$ ribosomes from the $50 \mathrm{~S}$ and $30 \mathrm{~S}$ subunits was accomplished by reverse-phase chromatography. The sample was loaded using a peristaltic pump into a self-packed Toyopearl Butyl (Tosoh Bioscience) column (200 ml) equilibrated in $80 \%$ Buffer C $-20 \%$ Buffer $D$ at $4^{\circ} \mathrm{C}$ attached to a BioLogic LP system (Bio-Rad). After the sample was loaded, the column was washed with 2 CV of Buffer C. The sample was eluted with a linear gradient from 20\% to 60\% Buffer D in 4.5 CV (900 ml) at a flow rate 6 $\mathrm{ml} / \mathrm{min}$. 75 fractions of $12 \mathrm{ml}$ each were collected. The absorbance at $260 \mathrm{~nm}$ was 
checked in fractions from 30 to 74 (every second fraction) using a Nanodrop 2000 (Thermo Scientific) (Fig. 4.5).

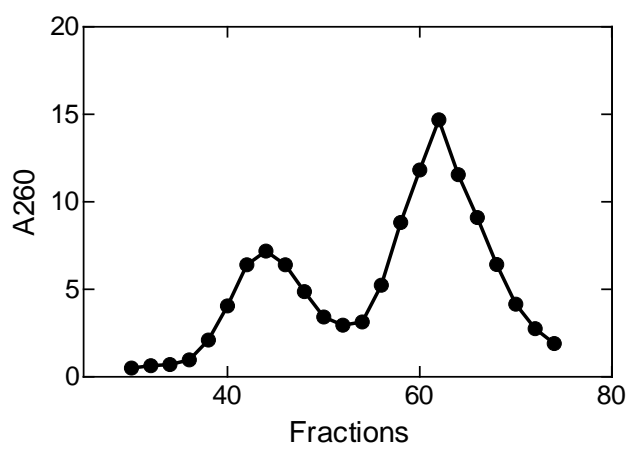

Figure 4.5 Typical separation profile of $T$. thermophilus ribosomes after the reverse-phase chromatography on the Toyopearl Butyl column. The absorbance of the chromatography fractions is measured at $260 \mathrm{~nm}$ by Nanodrop 2000 (Thermo Scientific). Fractions $1-29$ do not contain ribosomal material, therefore are not shown in this profile. The first peak (fractions $40-50$ ) corresponds to the $50 \mathrm{~S}$ subunits; the second peak (fractions $60-70$ ) corresponds to 705 ribosomes.

Fractions 58-66 were pooled together. The volume of the sample was increased to 180 $\mathrm{ml}$ with Buffer $\mathrm{E}$ to fill three Ti45 tubes. The concentration of $\mathrm{MgCl}_{2}$ in the sample was increased to $42 \mathrm{mM}$ by adding $1 \mathrm{M} \mathrm{MgCl}_{2}$ solution. The ribosomes were pelleted by centrifugation in a Beckman Ultracentrifuge using Ti45 rotor at $45000 \mathrm{rpm}, 4^{\circ} \mathrm{C}, 17 \mathrm{~h}$.

After the centrifugation step, the supernatant was discarded and each ribosomal pellet was dissolved in $1 \mathrm{ml}$ of Buffer $\mathrm{E}$ at $4^{\circ} \mathrm{C}$. The ribosome containing solutions were collected in one tube for sample homogenization. Sucrose gradients from $5 \%$ to $20 \%$ sucrose in Buffer E were prepared a day before using a Gradient Maker (Biocomp) and were left to equilibrate overnight at $4^{\circ} \mathrm{C}$. Ribosomal samples were then loaded on SW-28 Rotor tubes (Beckman Coulter) containing the sucrose gradients for further purification by centrifugation at $14500 \mathrm{rpm}$ at $4^{\circ} \mathrm{C}$ for $19 \mathrm{~h}$.

The sucrose gradients were fractionated after the centrifugation using a Bio-Rad Econo System (Bio-Rad Laboratories). The absorbance of each fraction was checked using the Nanodrop 2000 (Thermo Scientific) at $260 \mathrm{~nm}$. The typical profile after ribosomal purification on the sucrose gradients is depicted in Fig. 4.6 with the large peak corresponding to the $70 \mathrm{~S}$ ribosomes and the small peak, around fraction 20, 
corresponding to the remaining free $50 \mathrm{~S}$ subunits. Only the fractions containing $70 \mathrm{~S}$ ribosome were combined and pelleted by centrifugation using a Beckman Ti45 rotor at $45000 \mathrm{rpm}$ at $4^{\circ} \mathrm{C}$ for $19 \mathrm{~h}$.

The ribosomal pellet was dissolved in $400 \mu \mathrm{l}$ of Buffer $\mathrm{G}$ at $4^{\circ} \mathrm{C}$. The concentration of the ribosomes in the final preparation was determined by measuring the absorbance at 260 $\mathrm{nm}$ and using the corresponding extinction coefficient. The preparation was stored at $80^{\circ} \mathrm{C}$.

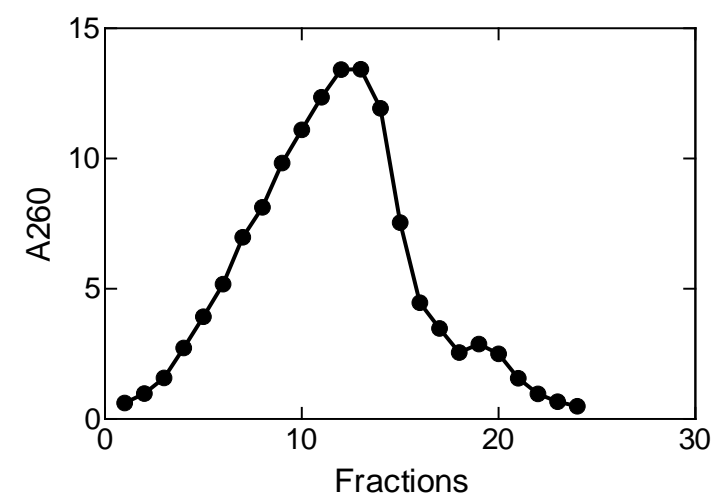

Figure 4.6 Typical separation profile of $T$. thermophilus ribosomes after the centrifugation in the sucrose gradient ( $5 \%-20 \%$ sucrose). The sucrose gradients were fractionated using BioRad Econo System (Bio-Rad Laboratories) and the absorbance of each fraction was checked on the Nanodrop (Thermo Scientific). The large peak corresponds to the $70 \mathrm{~S}$ ribosomes, the small peak at fraction 20 corresponds to the $50 \mathrm{~S}$.

\section{IF1 and IF3 purification.}

\section{Buffers.}

Lysis buffer: $50 \mathrm{mM}$ Hepes-KOH pH 7.5, $100 \mathrm{mM} \mathrm{KCl}, 10 \mathrm{mM} \mathrm{MgCl}_{2}$, 5\% glycerol, $1 \mathrm{mM}$ DTT, 1 mM Pefablock, Protease inhibitor cocktail 1 tablet $/ 25$ ml lysate, $1 \mu \mathrm{g} / \mathrm{ml}$ DNAse.

Buffer A: : 50 mM Hepes-KOH pH 7.5, 10 mM MgCl , 5 \% glycerol, 1 mM DTT, Buffer B: 50 mM Hepes-KOH pH 7.5, $1 \mathrm{M} \mathrm{KCl,} 10$ mM MgCl 2,5 \% glycerol, 1 mM DTT, Storage buffer: $50 \mathrm{mM}$ Tris- $\mathrm{HCl} \mathrm{pH} \mathrm{7.5;} 70 \mathrm{mM} \mathrm{NH}_{4} \mathrm{Cl}, 30 \mathrm{mM} \mathrm{KCl}, 5 \%$ glycerol, $1 \mathrm{mM}$ DTT. 
After $\mathrm{pH}$ adjustment all solutions were filtered through a $0.2 \mu \mathrm{m}$ cellulose acetate filter (Sartorius Stedim).

If required, DTT, Pefablock, Protease inhibitor cocktail and DNAse were added to the buffers right before use.

The vector $\mathrm{pET}-30 \mathrm{~b}$ containing infA or infC gene from $T$. thermophilus was transformed

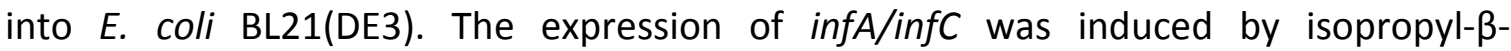
thiogalactopyranoside (IPTG) for $3 \mathrm{~h}$. The cells were harvested when $\mathrm{OD}_{600}$ reached 2.5. Two liters of culture yielded approximately $5 \mathrm{~g}$ of cells. Cells were resuspended in $20 \mathrm{ml}$ of lysis buffer and opened by sonication at $4^{\circ} \mathrm{C}$. The cell lysate was centrifuged to remove cell debris using a JA 25.50 rotor at $13000 \mathrm{rpm}$ at $4^{\circ} \mathrm{C}$ for $30 \mathrm{~min}$. The supernatant was incubated at $65^{\circ} \mathrm{C}$ for $20 \mathrm{~min}$. At this temperature, most of $E$. coli proteins are denatured. The suspension was cooled on ice for $30 \mathrm{~min}$ and centrifuged again using a JA 25.50 rotor at $13000 \mathrm{rpm}$ at $4^{\circ} \mathrm{C}$ for $30 \mathrm{~min}$. The supernatant containing T. thermophilus IF1/IF3 was cleared through a $0.2 \mu \mathrm{m}$ sterile filter. The protein solution was loaded into a HiTrap SP HP $5 \mathrm{ml}$ column equilibrated in 5 CV of Buffer A. After sample loading, the unbound material was washed with $5 \mathrm{CV}$ of Buffer $\mathrm{A}$. The sample was eluted with a gradient from $0 \%$ to $100 \%$ of Buffer B in $20 \mathrm{CV}$ at a flow rate of 2 $\mathrm{ml} / \mathrm{min}$. FPLC fractions were analyzed by SDS-PAGE and the fractions containing either IF1 or IF3 were dialyzed against Storage buffer. The preparations were flash frozen in liquid nitrogen and stored at $-80^{\circ} \mathrm{C}$.

\section{IF2 purification.}

Buffers.

Lysis buffer: $40 \mathrm{mM}$ Tris- $\mathrm{HCl} \mathrm{pH}$ 7.5, $10 \mathrm{mM} \mathrm{MgCl} 2,1 \mathrm{mM}$ DTT, $1 \mu \mathrm{g} / \mathrm{ml} \mathrm{PMSF}, 1 \mu \mathrm{g} / \mathrm{ml}$ DNAse.

Buffer A: 40 mM Tris-HCl pH 7.5, 0.1 mM EDTA, 1 mM DTT.

BufferB: 40 mM Tris-HCl pH 7.5, 0.1 mM EDTA, 1 mM DTT, $1 \mathrm{M} \mathrm{NaCl}$.

Buffer C: 40 mM Tris- $\mathrm{HCl}$ pH 7.5, 0.1 mM EDTA, 1 mM DTT, $1 \mathrm{M}(\mathrm{NH} 4)_{2} \mathrm{SO}_{4}$.

Buffer D: 40 mM Tris-HCl pH 7.5, 0.1 mM EDTA, 1 mM DTT. 
Storage buffer: $20 \mathrm{mM}$ Tris- $\mathrm{HCl} \mathrm{pH}$ 7.5, $50 \mathrm{mM} \mathrm{KCl}, 10 \mathrm{mM} \mathrm{NH}_{4} \mathrm{Cl}, 10 \mathrm{mM} \mathrm{MgAc}_{2}, 1 \mathrm{mM}$ DTT.

After $\mathrm{pH}$ adjustment all solutions were filtered through a $0.2 \mu \mathrm{m}$ cellulose acetate filter (Sartorius Stedim).

If required, DTT, PMSF and DNAse were added to the buffers right before use.

The vector $\mathrm{pET}-30 \mathrm{~b}$ containing infB gene from $T$. thermophilus was transformed into $E$. coli BL21(DE3). The cells were grown in LB medium containing kanamycin $30 \mu \mathrm{g} / \mathrm{ml}$. The culture was grown until the $\mathrm{OD}_{600}$ reached 0.8 , then the expression of the IF2 was induced by addition of $1 \mathrm{mM}$ IPTG. The cells from 2 I culture were harvested by centrifugation, resuspended in $20 \mathrm{ml}$ in Lysis buffer and opened by sonication. The cell lysate was centrifuged using a JA25.50 rotor at $13000 \mathrm{rpm}$ at $4^{\circ} \mathrm{C}$ for $30 \mathrm{~min}$ to eliminate cell debris. The supernatant was transferred to a fresh tube and centrifuged using a $\mathrm{Ti}$ 50.2 rotor at $50000 \mathrm{rpm}$ at $4^{\circ} \mathrm{C}$ for $2 \mathrm{~h}$ in order to clear the lysate of heavy molecular material. After this procedure, the lysate was loaded into a self-packed Q Sepharose 75 $\mathrm{ml}$ column (GE Healthcare) equilibrated in Buffer A. The unbound material was washed away with 2 bed volumes of Buffer $A$ and the sample was eluted with a gradient form $0 \%$ to $100 \%$ of Buffer $B$ in 12 bed volumes. The fractions were analyzed by SDS-PAGE and the IF2 containing fractions were pooled together, incubated for $20 \mathrm{~min}$ at $65^{\circ} \mathrm{C}$ for the heat denaturation of the $E$. coli proteins and then incubated on ice for $20 \mathrm{~min}$. The sample was centrifuged in a JA 25.50 rotor at $13000 \mathrm{rpm}$ at $4^{\circ} \mathrm{C}$ for $30 \mathrm{~min}$ to get rid of the formed precipitates. An ammonium sulfate $\left((\mathrm{NH} 4)_{2} \mathrm{SO}_{4}\right)$ solution was then added to the supernatant to a final concentration of $1 \mathrm{M}$ and the sample was centrifuged in a JA25.50 rotor at $13000 \mathrm{rpm}$ at $4^{\circ} \mathrm{C}$ for $30 \mathrm{~min}$. The supernatant was loaded into a HiPrep Phenyl FF 16/10 column (GE healthcare) equilibrated in buffer $C$. The unbound material was washed with $2 \mathrm{CV}$ of Buffer $\mathrm{C}$ and the sample was eluted in a gradient of $0 \%$ to $100 \%$ Buffer $D$ in $10 \mathrm{CV}$ at a flow rate $4 \mathrm{ml} / \mathrm{min}$. FPLC fractions were analyzed by SDSPAGE and the IF2 containing fractions were diluted in $3.8 \mathrm{M}(\mathrm{NH} 4)_{2} \mathrm{SO}_{4}$ solution to reach a final concentration of $1 \mathrm{M}$. Subsequently, the sample was loaded into a self-packed 20 $\mathrm{ml}$ Toyopearl Butyl column (Tosoh Bioscience) equilibrated in Buffer $\mathrm{C}$. The unbound material was washed with $2 \mathrm{CV}$ of Buffer $\mathrm{C}$ and the sample was eluted with a gradient from $25 \%$ to $80 \%$ Buffer D. The fractions were analyzed by SDS-PAGE and the IF2 containing fractions were combined and concentrated to $8 \mathrm{mg} / \mathrm{ml}$ in Storage Buffer. The preparation was flash frozen in liquid nitrogen and kept at $-80^{\circ} \mathrm{C}$. 


\section{Methionyl-tRNA ${ }^{\text {fMet }}$ synthethase.}

\section{Buffers.}

Lysis Buffer: 20 mM Tris-HCl pH 7.0, 30 mM KCl, 1 mM DTT, 1 g/ml PMSF, 0.1 mM BZA.

Superdex Buffer: 20 mM Tris- $\mathrm{HCl}$ pH 7.0, 30 mM KCl, 1 mM DTT.

Buffer A MonoQ: 20 mM Tris-HCl pH 7.0, 1 mM DTT.

Buffer B MonoQ: 20 mM Tris-HCl pH 7.0, 1 M KCl, 1 mM DTT.

Storage Buffer: 20 mM Tris-HCl pH 7.0, 30 mM KCl, 50 \% glycerol, 1 mM DTT.

After $\mathrm{pH}$ adjustment all solutions were filtered through a $0.2 \mu \mathrm{m}$ cellulose acetate filter (Sartorius Stedim).

If required, DTT, BZA and PMSF were added to the buffers right before use.

The pUC18Ftg plasmid overexpressing methionyl-tRNA synthethase from E. coli was kindly provided by Prof. Y. Mechulam (Fourmy et al., 1991; Hirel et al., 1988; Mellot et al., 1989). The methionyl-tRNA synthethase used in this work is not a full length enzyme, but a fully active truncated monomeric form of the $E$. coli methionyl-tRNA synthetase encompassing the $547 \mathrm{~N}$-terminal residues of the methionyl-tRNA synthethase (MTS547) (Fourmy et al., 1991; Mellot et al., 1989). The plasmid was transformed into $E$. coli strain JM101Tr. The cells were inoculated directly into $1 \mathrm{I}$ of 2xTY medium (16 g tryptone, $10 \mathrm{~g}$ yeast extract, $5 \mathrm{~g} \mathrm{NaCl}$ ) containing ampicillin $50 \mu \mathrm{g} / \mathrm{ml}$ and $1 \mathrm{mM}$ IPTG and grown overnight. Cells were harvested by centrifugation and the pellets were resuspended in $20 \mathrm{ml}$ of Lysis Buffer. The cells were opened by sonication and cell debris was pelleted via centrifugation using a JA 25.50 rotor at $15000 \mathrm{rpm}$ at $4^{\circ} \mathrm{C}$ for $10 \mathrm{~min}$. The supernatant was transferred to a new tube and one tenth of sample volume of a 30 $\%(w / v)$ streptomycin sulphate solution was added to the supernatant to precipitate nucleic acids. The solution was incubated at constant stirring at $4^{\circ} \mathrm{C}$ for $30 \mathrm{~min}$. The precipitates were pelleted via centrifugation using a JA 25.50 rotor at $15000 \mathrm{rpm}$ at $4^{\circ} \mathrm{C}$ for $10 \mathrm{~min}$. The supernatant was transferred to a new tube and $\left(\mathrm{NH}_{4}\right)_{2} \mathrm{SO}_{4}$ solution was added to the sample to $80 \%$ of saturation and left at constant stirring at $4^{\circ} \mathrm{C}$ for $30 \mathrm{~min}$. The sample was centrifuged using a JA 25.50 rotor at $15000 \mathrm{rpm}$ at $4^{\circ} \mathrm{C}$ for $10 \mathrm{~min}$. The supernatant was discarded, the pellet was dissolved in $3 \mathrm{ml}$ of the Superdex Buffer and 
dialyzed against $3 \mathrm{I}$ of fresh Superdex Buffer to get rid of the residual $\left(\mathrm{NH}_{4}\right)_{2} \mathrm{SO}_{4}$. The sample was further purified by gel filtration using a $200 \mathrm{ml}$ column self-packed with Superdex 200 prep grade resin (Sigma-Aldrich). The gel filtration was performed using Superdex buffer. The fractions containing MTS5547 were collected and loaded tino a MonoQ HR 5/5 column (GE Healthcare) equilibrated in 3\% Buffer B MonoQ. The sample was eluted using a gradient from $3 \%$ to $30 \%$ of Buffer B MonoQ in $20 \mathrm{CV}$ at a flow rate of $1 \mathrm{ml} / \mathrm{min}$. Fractions of $0.5 \mathrm{ml}$ were collected. The fractions containing the protein of interest were combined and concentrated to $50 \mathrm{mg} / \mathrm{ml}$. Finally, the sample was dialyzed into the storage buffer using Mini Dialysis Devices (Thermo Scientific), flash frozen and stored at $-80^{\circ} \mathrm{C}$.

\section{Methionyl-tRNA ${ }^{\text {fMet }}$ formyltransferase.}

\section{Buffers.}

Lysis Buffer: $20 \mathrm{mM}$ Tris- $\mathrm{HCl} \mathrm{pH}$ 7.0, $100 \mathrm{mM} \mathrm{KCl}, 1 \mathrm{mM}$ DTT, $1 \mu \mathrm{g} / \mathrm{ml}$ PMSF, $0.1 \mathrm{mM}$ BZA.

Superdex Buffer: 20 mM Tris- $\mathrm{HCl}$ pH 7.0, 30 mM KCl, 1 mM DTT.

Buffer A: 20 mM Tris-HCl pH 7.0, 1 mM DTT.

Buffer B: 20 mM Tris- $\mathrm{HCl}$ pH 7.0, $1 \mathrm{M} \mathrm{KCl,} 1$ mM DTT.

Storage Buffer: 20 mM Tris- $\mathrm{HCl}$ pH 7.0, $30 \mathrm{mM} \mathrm{KCl,} 50$ \% glycerol, $1 \mathrm{mM}$ DTT.

After $\mathrm{pH}$ adjustment all solutions were filtered through a $0.2 \mu \mathrm{m}$ cellulose acetate filter (Sartorius Stedim).

If required, DTT, BZA, PMSF and DNAse were added to the buffers right before use.

The pUC18Ftg plasmid overexpressing methionyl-tRNA ${ }^{\text {fMet }}$ formyltransferase (MFT) from E. coli was kindly provided by Prof. Y. Mechulam (Schmitt et al., 1996a, 1996b, 1999). The plasmid was transformed into E. coli strain JM101Tr. The cells were grown in the $2 x T Y$ medium containing ampicillin $50 \mu \mathrm{g} / \mathrm{ml}$. When the $\mathrm{OD}_{600}$ of the culture reached the 0.9 , the expression of the MFT was initiated by addition of $0.3 \mathrm{mM}$ IPTG. The cells were grown for $2 \mathrm{~h}$ after induction until the $\mathrm{OD}_{600}$ of the culture reached 1.85 . The cells 
were harvested by centrifugation and the pellets were resuspended in $20 \mathrm{ml}$ of Lysis Buffer. The cells were opened by sonication and the cell lysate was centrifuged using a JA 25.50 rotor at $15000 \mathrm{rpm}, 4^{\circ} \mathrm{C}$ for $10 \mathrm{~min}$. The supernatant was transferred to a new tube and $1 / 10$ of the sample volume of a $30 \%(w / v)$ streptomycin sulphate solution was added. The solution was incubated at $4^{\circ} \mathrm{C}$ at constant stirring for $20 \mathrm{~min}$ for nucleic acid precipitation and centrifuged using a JA 25.50 rotor at $15000 \mathrm{rpm}, 4^{\circ} \mathrm{C}$ for $10 \mathrm{~min}$. A $\left(\mathrm{NH}_{4}\right)_{2} \mathrm{SO}_{4}$ solution was then added to the sample to $45 \%$ of saturation and incubated at constant stirring at $4^{\circ} \mathrm{C}$ for $20 \mathrm{~min}$. The sample was centrifuged using a JA 25.50 rotor at $15000 \mathrm{rpm}$ at $4^{\circ} \mathrm{C}$ for $30 \mathrm{~min}$. The supernatant was transferred to a new tube, $\left(\mathrm{NH}_{4}\right)_{2} \mathrm{SO}_{4}$ was again added to the sample to $70 \%$ of saturation and incubated at constant stirring at $4^{\circ} \mathrm{C}$ for $20 \mathrm{~min}$. The sample was centrifuged using a JA 25.50 rotor at $15000 \mathrm{rpm}$ at $4^{\circ} \mathrm{C}$ for $30 \mathrm{~min}$. The pellet was dissolved in $2 \mathrm{ml}$ of Superdex buffer. The sample was loaded into a $200 \mathrm{ml}$ gel filtration column self-packed with Superdex 200 prep grade (Sigma-Aldrich). Gel filtration was performed using the Superdex Buffer. Gel filtration fractions were collected and analyzed by SDS-PAGE. The fractions containing the protein of interest were combined. MFT was further purified using a $8 \mathrm{ml}$ column self-packed with Q Sepharose (GE Healthcare) equilibrated in $3 \%$ Buffer B. The sample was eluted using a gradient from $3 \%$ to $50 \%$ Buffer B in $20 \mathrm{CV}$. The fractions were analyzed by the SDS-PAGE and MFT containing fractions were pooled and loaded into a MonoQ HR 5/5 column (GE Healthcare) equilibrated in 3\% Buffer B MonoQ. The sample was eluted using a gradient from $3 \%$ to $30 \%$ of Buffer B MonoQ in $20 \mathrm{CV}$ at a flow rate of $1 \mathrm{ml} / \mathrm{min}$. Fractions of $0.5 \mathrm{ml}$ were collected. The fractions containing the MFT were combined and concentrated to $43 \mathrm{mg} / \mathrm{ml}$. Glycerol was added to the sample to a final concentration of $50 \%$. The final MFT preparation was flash frozen in liquid nitrogen and stored at $-80^{\circ} \mathrm{C}$.

\section{tRNA $^{\text {fMet }}$}

Buffers.

Buffer T1: $1 \mathrm{mM}$ Tris- $\mathrm{HCl} \mathrm{pH}$ 7.5, $10 \mathrm{mM} \mathrm{MgAc}$.

Buffer T2: 20 mM Tris- $\mathrm{HCl}$ pH 7.5, 8 mM MgCl $2,0.1$ mM EDTA, $0.2 \mathrm{M} \mathrm{NaCl}$.

Buffer A: 20 mM Tris- $\mathrm{HCl}$ pH 7.5, 8 mM MgCl $2,0.1$ mM EDTA.

Buffer B: 20 mM Tris- $\mathrm{HCl}$ pH 7.5, 8 mM MgCl $2,0.1$ mM EDTA, $1 \mathrm{M} \mathrm{NaCl}$. 
After $\mathrm{pH}$ adjustment all solutions were filtered through a $0.2 \mu \mathrm{m}$ cellulose acetate filter (Sartorius Stedim).

The plasmid pBStRNAfMetY2 overexpressing tRNA ${ }^{\text {fMet }}$ from E. coli was kindly provided by Prof. Y. Mechulam (Mechulam et al., 2007). The plasmid was transformed into E. coli strain JM101Tr. The cells were inoculated directly to 1 I flasks containing 2x TY medium and ampicillin $50 \mu \mathrm{g} / \mathrm{ml}$ for $24 \mathrm{~h}$. The cells were harvested by centrifugation and resuspended in Buffer T1 ( $1 \mathrm{ml}$ of Buffer T1 per gram of cells). An equal volume of phenol saturated in Buffer T1 was added to the cell suspension and mixed by vortexing for $3 \mathrm{~min}$. The cell lysate was centrifuged using a JLA 25.50 rotor at $15000 \mathrm{rpm}$ at $20^{\circ} \mathrm{C}$ for $30 \mathrm{~min}$. The aqueous phase was transferred to a new tube. The ionic strength of the sample was increased by adding $\mathrm{NaCl}$ to a final concentration of $0.5 \mathrm{M}$. The RNA was precipitated by addition of 2.2 volumes of ice-cold ethanol. The sample was mixed on vortex for $10 \mathrm{~s}$ and centrifuged using a JA 25.50 rotor at $15000 \mathrm{rpm}, 4^{\circ} \mathrm{C}$ for $30 \mathrm{~min}$. The pellet was dissolved in $5 \mathrm{ml}$ of $1 \mathrm{M} \mathrm{NaCl}$ and centrifuged at $15000 \mathrm{rpm}$ at $4^{\circ} \mathrm{C}$ for $30 \mathrm{~min}$ to get rid of non-dissolved material. The supernatant was transfered to a new tube and the tRNA was precipitated by adding of 2.2 volumes of ice-cold ethanol and further centrifugation at $15000 \mathrm{rpm}$ at $4^{\circ} \mathrm{C}$ for $30 \mathrm{~min}$. The pellet was dried in the lyophilic dryer for $30 \mathrm{~min}$. The dry sample was dissolved in $2 \mathrm{ml}$ of $1.8 \mathrm{M}$ Tris- $\mathrm{HCl} \mathrm{pH} 8.0$ and incubated at $37^{\circ} \mathrm{C}$ for $90 \mathrm{~min}$ for tRNA deacylation. After the deacylation step, $\mathrm{NaCl}$ was added to a final concentration of $0.5 \mathrm{M}$ to increase the ionic strength and tRNA was precipitated by adding of 2.2 volumes of ice-cold ethanol. The precipitation was done at $-20^{\circ} \mathrm{C}$ overnight. The tRNA was pelleted by centrifugation in a JA25.50 rotor at $15000 \mathrm{rpm}$ at $4^{\circ} \mathrm{C}$ for $20 \mathrm{~min}$. The pellet was dried and dissolved in $5 \mathrm{ml}$ of Buffer T2. The tRNA was further purified using a $25 \mathrm{ml}$ column self-packed with $Q$ Sepharose resin (GE Healthcare) equilibrated in $20 \%$ Buffer $A$. The unbound material was washed with $1 \mathrm{CV}$ of $20 \%$ Buffer $A$. The sample was eluted at a rate $2 \mathrm{ml} / \mathrm{min}$ with a gradient starting from $36 \%$ to $38 \%$ Buffer $B$ in $5 \mathrm{CV}$ and then with a isocratic flow step at the concentration of $38 \%$ Buffer $B$ for $1 \mathrm{~h}$. Fractions of $4 \mathrm{ml}$ were collected. The fractions containing tRNA ${ }^{\text {fMet }}$ were combined; $\mathrm{NaCl}$ was added to a final concentration of $0.5 \mathrm{M}$ and tRNA was precipitated by addition of 2.2 volumes of ice-cold ethanol at $-20^{\circ} \mathrm{C}$ overnight. The tRNA ${ }^{\mathrm{fMet}}$ was pelleted by centrifugation in a JA 25.50 rotor at $15000 \mathrm{rpm}$ at $4^{\circ} \mathrm{C}$ for 30 min. The pellet was dried in the lyophilic dryer and dissolved in $300 \mu$ of deionized water. The preparation was flash frozen and kept at $-80^{\circ} \mathrm{C}$. 


\section{Preparation of formyl-donor.}

Folinic acid (Sigma-Aldrich) is used as a formyl-donor precursor. $25 \mathrm{~g}$ of folinic acid were dissolved in $2 \mathrm{ml}$ of $50 \mathrm{mM}$ 2-mercaptoethanol and $0.22 \mathrm{ml}$ of $1 \mathrm{~N} \mathrm{HCl}$ and incubated at room temperature for $4 \mathrm{~h}$. The maximum absorbance of the folinic acid is at $290 \mathrm{~nm}$, whereas the maximum absorbance of the formyl donor is at $350 \mathrm{~nm}$. Therefore, the amount of formyl donor formed in the reaction was monitored at $350 \mathrm{~nm}$ every hour. After $4 \mathrm{~h}$, the reaction was completed. The sample was diluted 6 times with water and the $\mathrm{pH}$ was adjusted to 7.5 with $2 \mathrm{M}$ Tris-base $\mathrm{pH} 11$. The preparation was kept at $-20^{\circ} \mathrm{C}$.

\section{Aminoacylation and formylation of tRNA ${ }^{\text {fMet }}$.}

\section{Buffers}

Buffer for aminoacylation: $30 \mathrm{mM}$ imidazole- $\mathrm{HCl} \mathrm{pH} \mathrm{7.5,} 100 \mathrm{mM} \mathrm{KCl}, 10 \mathrm{mM} \mathrm{MgAc}, 1$ mM DTT.

Buffer A: $50 \mathrm{mM}$ potassium phosphaste $\mathrm{pH}$ 7.0.

Buffer B: $50 \mathrm{mM}$ potassium phosphaste $\mathrm{pH}$ 7.0, $1.7 \mathrm{M}\left(\mathrm{NH}_{4}\right)_{2} \mathrm{SO}_{4}$.

Buffer C: $50 \mathrm{mM}$ sodium acetate $\mathrm{pH}$ 5.0.

Buffer D: $50 \mathrm{mM}$ sodium acetate $\mathrm{pH}$ 5.0, $1 \mathrm{M} \mathrm{NaCl}$.

After $\mathrm{pH}$ adjustment all solutions were filtered through a $0.2 \mu \mathrm{m}$ cellulose acetate filter (Sartorius Stedim).

If required, DTT was added to the buffers right before use.

The reaction of tRNA $^{\text {fMet }}$ aminoacylation and formylation was performed in aminoacylation buffer. An amount corresponding to 20 optical units of tRNA ${ }^{\text {fMet }}$ was used in the reaction. The following concentrations were used: $0.1 \mu \mathrm{g} / \mu \mathrm{l}$ methionyltRNA $^{\text {fMet }}$ synthethase, $5 \mathrm{mM}$ ATP, $5 \mathrm{mM}$ phosphoenolpyruvate, $0.025 \mathrm{mg} / \mathrm{ml}$ pyruvate kinase, $80 \mu \mathrm{M}$ methionine, $0.1 \mu \mathrm{g} / \mu \mathrm{l}$ methionyl-tRNA ${ }^{\text {fMet }}$ formyltransferase, $0.3 \mathrm{mM}$ formyl donor. The total volume of the reaction was $1.5 \mathrm{ml}$. The reaction was incubated at $30^{\circ} \mathrm{C}$ for $50 \mathrm{~min}$. Aminoacylation was stopped by adding $1 / 10$ of volume of $20 \%$ potassium acetate solution at $\mathrm{pH}$ 5.0. $\mathrm{fMet}^{\mathrm{tRNA}}{ }^{\mathrm{fMet}}$ phenol extraction was performed 92 
by adding of an equal volume of water saturated phenol, mixing on the vortex and centrifuging at $5000 \mathrm{rpm}$ at $4^{\circ} \mathrm{C}$ for $5 \mathrm{~min}$. The aqueous phase was transfered to a new tube. Extraction of the remaining tRNA from the phenol phase was done by adding once again an equal volume of buffer for aminoacylation and repeating the vortexing and centrifugation step. The aqueous phases of each extraction step were combined for further steps. Potassium acetate $\mathrm{pH} 5.0$ was added to the extracted fMet-tRNA ${ }^{\text {fMet }}$ to a final concentration of $2 \%$. fMet-tRNA ${ }^{\text {fMet }}$ was precipitated with 2.2 volumes of ice-cold ethanol at $-20^{\circ} \mathrm{C}$ overnight. The tRNA was pelleted by centrifugation at $13000 \mathrm{rpm}$ at $4^{\circ} \mathrm{C}$ for $30 \mathrm{~min}$. The pellet was washed twice in ice-cold $70 \%$ ethanol and dried in a Speedvac Concentrator (Savant) for $4 \mathrm{~min}$. The tRNA pellet was dissolved in $1 \mathrm{ml}$ of Buffer $\mathrm{A}$ and filtered through the $0.2 \mu \mathrm{M}$ centrifugal filter units. The tRNA solution was further purified using a TSKgel phenyl $-5 \mathrm{PW}(7.5 \mathrm{~cm} \times 7.5 \mathrm{~mm})$ column (Tosoh Bioscience) equilibrated in Buffer A. fMet-tRNA ${ }^{\mathrm{fMet}}$, Met-tRNA ${ }^{\mathrm{fMet}}$ and $\mathrm{TRNA}^{\mathrm{fMet}}$ were separated on the TSKgel phenyl-5PW column using a gradient from $0 \%$ to $100 \%$ of Buffer $B$ in $99 \mathrm{ml}$ at a flow rate of $0.7 \mathrm{ml} / \mathrm{min}$. The fractions containing $\mathrm{fMet}_{\mathrm{N}} \mathrm{tRNA} \mathrm{fMet}^{\mathrm{f}}$ were combined and concentrated to $4 \mathrm{ml}$ in Buffer C. The sample was loaded into a MonoQ HR 5/5 (GE Healthcare) equilibrated in Buffer $C$. The unbound sample was washed with $5 \mathrm{CV}$ of Buffer $A$ and the sample was eluted with a gradient from $0 \%$ to $100 \%$ Buffer B in $20 \mathrm{CV}$. The fMet-tRNA ${ }^{\text {fMet }}$ was precipitated by ice-cold ethanol and pelleted by centrifugation. The pellets were air-dried and dissolved in water. The preparation was kept at $-80^{\circ} \mathrm{C}$.

\section{Crystallization trials.}

To identify initial crystallization conditions, available home-made crystallization reagents were screened in 96-well MRC plates by sitting drop vapor diffusion technique. Drops of $1000 \mathrm{nl}$ (500 nl protein solution $+500 \mathrm{nl}$ reservoir) were dispensed using a TTP Mosquito Crystallization Robot (TTP LabTech). Manual setups in a 24-well format also using the sitting drop vapor diffusion technique were performed by addition of $2 \mu$ l reservoir solution to $2 \mu \mathrm{l}$ of the sample. Crystallization plates were kept at $22^{\circ} \mathrm{C}$.

\section{Fluorescent labeling of IF2.}

Atto488 labeling buffer: $130 \mathrm{mM}$ Hepes-KOH pH 8.1, $150 \mathrm{mM} \mathrm{NaCl}$.

Mant labeling buffer: 5 mM Hepes- $\mathrm{KOH}$ pH 7.5, $70 \mathrm{mM} \mathrm{NH}_{4} \mathrm{Cl}, 30 \mathrm{mM} \mathrm{KCl}$. 
IF2 storage buffer: $10 \mathrm{mM}$ Tris- $\mathrm{HCl} \mathrm{pH} 7.5,10 \mathrm{mM} \mathrm{MgAc}_{2}, 50 \mathrm{mM} \mathrm{KCl}, 10 \mathrm{mM} \mathrm{NH}_{4} \mathrm{Cl}, 1$ mM DTT, 5 \% glycerol.

IF2 was labeled with either Atto488 (ATTO-TEC GmbH, Siegen, Germany) or mant (Jena Bioscience, Jena, Germany) fluorescent dyes. The labeling dye Atto 488 was added to the IF2 in labeling buffer to a final concentration of $140 \mu \mathrm{M}$. The labeling procedure with mant dye was done using the Mant Protein Labeling Kit (Jena Bioscience) according to manufacturer's instructions. The labeling reactions were carried out for $1 \mathrm{~h}$ at room temperature in the dark at constant shaking. After $1 \mathrm{~h}$, the reaction was stopped by removing the excess of the dye using the Dye Removal Columns (Thermo Scientific). The buffer was exchanged for storage buffer using the ZEBA Spin Desalting Columns 7 MWCO (Thermo Scientific).

\section{Nitrocellulose filter binding assay .}

Nitrocellulose filters (Sartorius, Germany) were equilibrated in the $\mathrm{TAKM}_{7}$ buffer (50 $\mathrm{mM}$ Tris- $\mathrm{HCl} \mathrm{pH} \mathrm{7.5,} 70 \mathrm{mM} \mathrm{NH}_{4} \mathrm{Cl}, 30 \mathrm{mM} \mathrm{KCl}, 7 \mathrm{mM} \mathrm{MgCl}_{2}$ ) before use. $50 \mu \mathrm{l}$ of the mixture containing initiation complexes were applied to the nitrocellulose filter, spreading the mixture evenly around the filter. The filter was washed from the unbound components with $3 \mathrm{ml}$ of the ice-cold $\mathrm{TAKM}_{7}$ buffer. Filters were dissolved in $10 \mathrm{ml}$ of scintillation cocktail Quickscint 361 (Zinsser Analytic) and the radioactivity in the samples was measured by liquid scintillation counting. The amounts of $\left[{ }^{3} \mathrm{H}\right] \mathrm{fMet}$-tRNA ${ }^{\text {fMet }}$ bound to the ribosome was calculated using the specific activity of $\left[{ }^{3} \mathrm{H}\right] \mathrm{fMet}-\mathrm{tRNA} \mathrm{fMet}^{\mathrm{f}}$. The efficiency of the initiation complex formation was calculated as the molar ratio between tRNA, bound to the filter, and the $30 \mathrm{~S}$ subunit added into the mixture.

\section{GTPase reaction.}

IF2 GTPase reaction was performed by hand in TAKM 7 buffer $(50 \mathrm{mM}$ Tris $-\mathrm{HCl}$ pH 7.5, 70 $\mathrm{mM} \mathrm{NH}{ }_{4} \mathrm{Cl}, 30 \mathrm{mM} \mathrm{KCl}, 7 \mathrm{mM} \mathrm{MgCl}_{2}$ ) for $E$. coli system and in TAKM 10 buffer ( $50 \mathrm{mM}$ Tris- $\mathrm{HCl} \mathrm{pH} \mathrm{7.5,} 70 \mathrm{mM} \mathrm{NH}_{4} \mathrm{Cl}, 30 \mathrm{mM} \mathrm{KCl}, 10 \mathrm{mM} \mathrm{MgCl}_{2}$ ) for $T$. thermophilus system using fluorescent mant-GTP. The GTPase assay is described in detail in the Materials and Methods of Chapter 2 of the present work. 


\section{Gel filtration of the initiation complexes.}

Analytical gel filtration of the initiation complexes was performed on a Biosuite $4508 \mu \mathrm{m}$ HR SEC 7.8X300mm (Waters) column. The initiation complexes were formed at various conditions, as described in the Results section, and loaded into a gel filtration column to separate the ribosomal complexes from free factors, $\left[{ }^{3} H\right] f M e t-t R N A^{f M e t}, m R N A$ and GTP. The gel filtration was performed in TAKM 7 buffer at a flow rate of $0.7 \mathrm{ml} / \mathrm{min}$ for $30 \mathrm{~min}$. The fluorescent compounds were detected by the flow-through detector (Waters 2475 Multiwavelength Fluorescent Detector) and the ribosome was detected by the absorbance at $260 \mathrm{~nm}$ using the Waters 2487 Dual Absorbance Detector. Fractions of $0.7 \mathrm{ml}$ were collected. The radioactivity associated to $\left[{ }^{3} \mathrm{H}\right] \mathrm{fMet}-\mathrm{tRNA}{ }^{\mathrm{fMet}}$ was determined using liquid-liquid scintillation counting in $2 \mathrm{ml}$ of scintillation cocktail Lumasafe Plus (Perkin Elmer).

\section{Analytical sucrose gradients.}

Buffers.

Buffer A 10x: 100 mM Hepes-KOH pH 7.5, 500 mM KCl, $100 \mathrm{mM} \mathrm{NH}_{4} \mathrm{Cl}, 100 \mathrm{mM} \mathrm{MgAc}_{2}$, $10 \mathrm{mM}$ DTT.

Sucrose Buffer 1: $10 \%$ sucrose, 1 x Buffer A.

Sucrose Buffer 2: $30 \%$ sucrose, 1 x Buffer A.

After $\mathrm{pH}$ adjustment all solutions were filtered through a $0.2 \mu \mathrm{m}$ cellulose acetate filter (Sartorius Stedim).

The gradients were prepared from sucrose Buffer 1 and sucrose Buffer 2 using a Gradient Maker (Bio-Rad). The samples contained either $44 \mu \mathrm{M} 70 \mathrm{~S}$ alone or $70 \mathrm{~S}$ incubated with IF3 in 1:1 and 1:5 ratios. The total volume of reaction was $20 \mu$ l. All the samples were incubated at $55^{\circ} \mathrm{C}$ for $15 \mathrm{~min}$ and then loaded into the gradients. The ribosomal subunits were separated via centrifugation in a SW40 Ti rotor at $19500 \mathrm{rpm}$ at $4^{\circ} \mathrm{C}$ for $20 \mathrm{~h}$. The gradients were unloaded using a peristaltic pump P1 (GE Healthcare) at a rate $0.5 \mathrm{ml} / \mathrm{min}$ (rate 2 on the pump scale) and fractions of $0.5 \mathrm{ml}$ were collected. The absorbance of the fractions was measured at $260 \mathrm{~nm}$ on the Nanodrop 2000 (Thermo Scientific). 


\section{Sucrose cushion.}

Buffers.

$\mathrm{HAKM}_{7}$ 10x: 500 mM Hepes-KOH pH 7.5, $300 \mathrm{mM} \mathrm{KCl,} 700 \mathrm{mM} \mathrm{NH}_{4} \mathrm{Cl}, 70 \mathrm{mM} \mathrm{MgCl}_{2}, 10$ mM DTT.

Sucrose cushion: $1 \times \mathrm{HAKM}_{7}, 30 \%$ sucrose.

$500 \mu \mathrm{l}$ of sucrose cushion were laid into Beckman transparent tubes for the TLS55 rotor. $1.5 \mathrm{ml}$ of the $70 \mathrm{~S}$ IC $\left(0.2 \mu \mathrm{M}\right.$ 70S, $0.5 \mu \mathrm{M}$ IF1, $0.5 \mu \mathrm{M}$ IF2, $0.5 \mu \mathrm{M}$ IF3, $0.5 \mu \mathrm{M}\left[{ }^{3} \mathrm{H}\right.$ ]fMettRNA ${ }^{\text {fMet }}, 0.5 \mu \mathrm{M}$ mRNA, $10 \mu \mathrm{M}$ GTP) was formed for $20 \mathrm{~min}$ at $37^{\circ} \mathrm{C}$. The 70 I IC sample was overlaid on the sucrose cushion and centrifuged in a TLS55 rotor at $55000 \mathrm{rpm}$ at $4^{\circ} \mathrm{C}$ for $2 \mathrm{~h}$. The pellets were dissolved in $20 \mu$ of $\mathrm{HAKM}_{7}$ buffer. The concentration of the purified 70S IC was measured by absorbance of a 500x diluted sample at $260 \mathrm{~nm}$. Radioactivity contained in the $2 \mu \mathrm{l}$ of the sample was counted by liquid-liquid scintillation in $2 \mathrm{ml}$ of scintillation cocktail Lumasafe Plus (Perkin Elmer).

\section{Puromycin extraction.}

All the components of the 70S IC were mixed together except 70S. The ribosome was simultaneously added in the last round with puromycin. The reaction was incubated at $37^{\circ} \mathrm{C}$ for $20 \mathrm{~min}$. The reaction was stopped by addition of $500 \mu \mathrm{l}$ of PuroStopper solution (1.5 $\mathrm{M}$ sodium acetate, saturated solution of $\mathrm{MgSO}_{4}, \mathrm{pH} 4.5$ ). Subsequently, $800 \mu \mathrm{l}$ of ethyl acetate was added. $\left[{ }^{3} \mathrm{H}\right]$ methionine-puromycin was extracted to the ethyl acetate phase by intensive vortexing of the mixture for $2 \mathrm{~min}$. The phases were separated by centrifugation for $5 \mathrm{~min}$ at $15000 \mathrm{rpm}$ at $20^{\circ} \mathrm{C}$ in a benchtop centrifuge. $600 \mu \mathrm{l}$ of the ethyl acetate phase were sampled for counting the radioactivity associated with $\left[{ }^{3} \mathrm{H}\right]$ methionine-puromycin by liquid-liquid scintillation counting. $2 \mathrm{ml}$ of scintillation cocktail Lumasafe Plus (Perkin Elmer) were added to each sample.

\section{Results.}

\section{S $\Delta$ L9 characterization.}

For crystallization of the $70 S$ IC, we made use of mutant $70 S \Delta L 9$ ribosomes $T$. thermophilus HB8 strain. These ribosomes lack the large ribosomal subunit protein L9 (Selmer et al., 2012). In the wild type (WT) ribosomes, the preferred crystal packing 
involves protein L9 thus preventing binding of translational GTPases to the ribosome (Selmer et al., 2012). In order to solve this problem, a number of ribosomal complexes containing translational GTPases (EF-G, EF-Tu, RF3) were successfully crystalized using the 70S $\Delta$ L9 mutant (Gao et al., 2009; Jin et al., 2011; Schmeing et al., 2009; Voorhees et al., 2010).

The mutant 70S was purified using the same approach as the as the WT 70S is purified for crystallization. The quality of the preparation of the 70S $\Delta \mathrm{L} 9$ was compared to the WT $70 \mathrm{~S}$ preparation by ribosome profiling using sucrose gradients (Fig. 4.7, A). The migration behavior of the 70S $\Delta \mathrm{L} 9$ ribosomal subunits in the agarose gel was also compared to the WT 70 S (Fig. 4.7, B). The standard purification method was found to yield equally homogenous preparations suitable for crystallization of WT and mutant ribosomes .
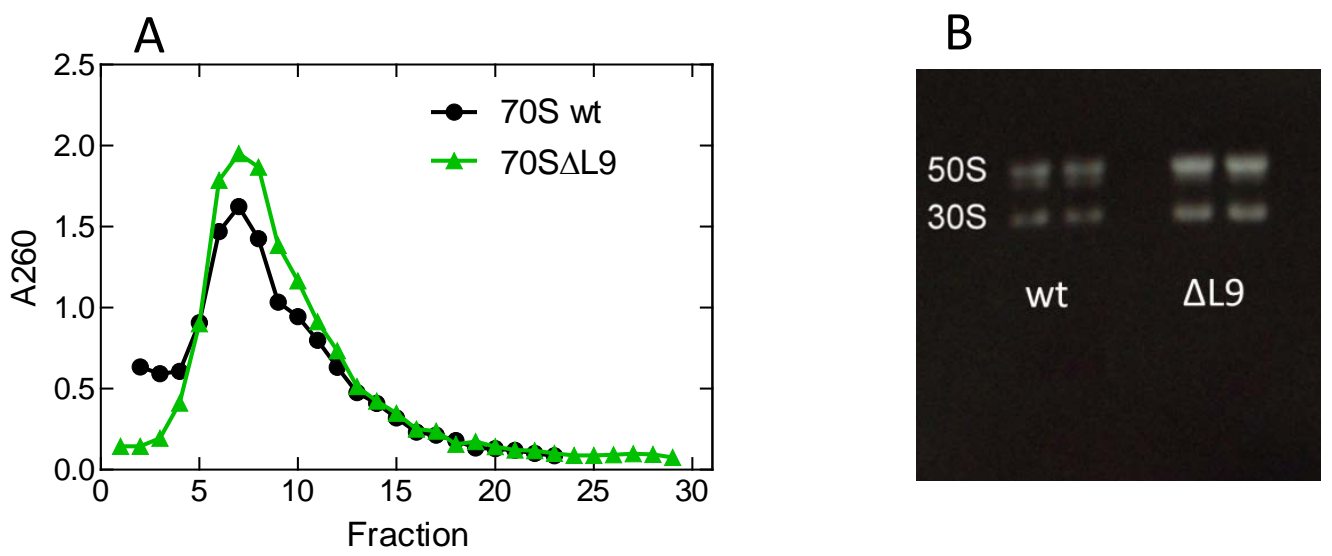

Figure 4.7 (A) 70 s profiling in the sucrose gradient of from $5 \%$ to $20 \%$ sucrose. Comparison of $70 \mathrm{~S} \Delta \mathrm{L} 9$ (green) to the $70 \mathrm{~S}$ wt (black). (B) Comparison of migration behavior of the 70S $\Delta \mathrm{L} 9$ to $70 \mathrm{~S}$ wt in the $1 \%$ agarose gel.

We have also analyzed whether the $70 \mathrm{~S} \Delta \mathrm{L} 9$ preparation is suitable for crystallization at known conditions. We managed to obtain needle crystals for the vacant 70S $\Delta$ L9 and large crystals when $70 \mathrm{~S} \Delta \mathrm{L} 9$ was cocrystallized with tRNA ${ }^{\mathrm{fMet}}$ (Fig. 4.8). Therefore, we concluded that the material is suitable for further crystallization experiments. 
A

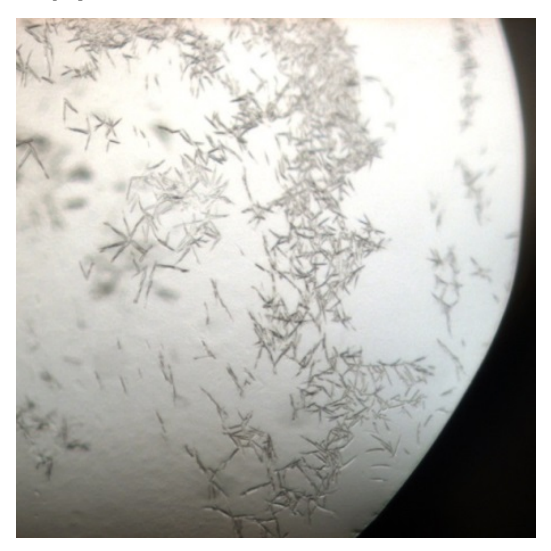

B

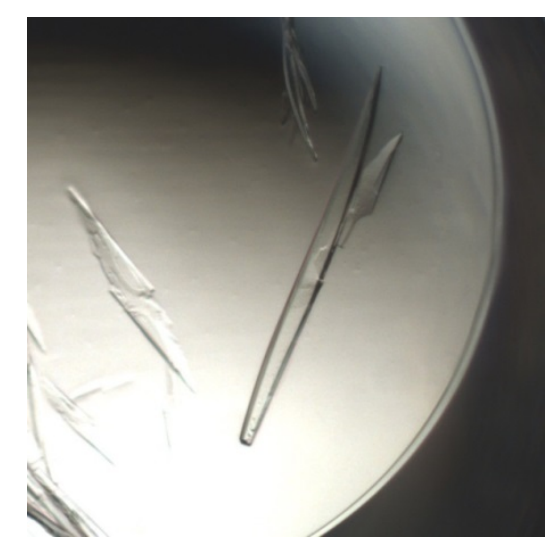

Figure 4.8 (A) Needle crystals of the vacant $70 S \Delta L 9$ (B) Crystals of $70 S \Delta$ L9 cocrystallized with tRNA ${ }^{\text {fMet }}$.

\section{IF2 purification for crystallization.}

IF2 from T. thermophilus was successfully expressed in E. coli and purified. Proteins from thermal organisms withstand higher temperatures than proteins from mesophilic organisms. Therefore, an important step of the purification procedure is the heat denaturation at $55^{\circ} \mathrm{C}$. Most of the $E$. coli proteins are precipitated at this step. The IF2 preparation obtained this way is of high purity which makes it suitable for crystallization (Fig. 4.9).

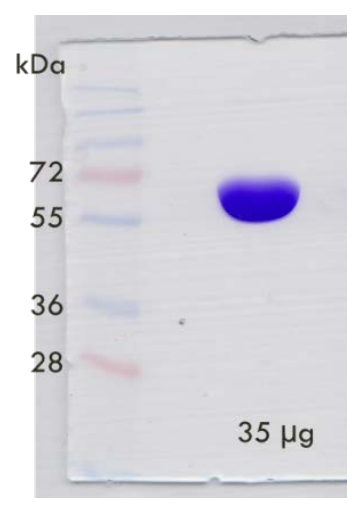

Figure 4.9 IF2 from $T$. thermophlilus expressed and purified from E. coli.

\section{IC crystallization trials.}

First, we tried 70S IC preparation for crystallization without adding IF1 and IF3 as we wanted to avoid additional 70S IC purification steps. 70S IC (3.6 $\mu \mathrm{M}$ 70S, $5.4 \mu \mathrm{M}$ IF2, 7.2 $\mu \mathrm{M}$ fMet-tRNA ${ }^{\mathrm{fMet}}, 7.2 \mu \mathrm{M}$ mRNA, $400 \mu \mathrm{M}$ GDPNP) was prepared by mixing all the 
components together in Buffer $G$ (see Materials and Methods section, 70S $\Delta$ L9 preparation) and incubating at $37^{\circ} \mathrm{C}$ for $30 \mathrm{~min}$. Subsequently, special non-ionic detergent (Deoxy Big Chap, Hampton Research) was added and the mixture was further incubated for $30 \mathrm{~min}$ at room temperature. The mixture was then pipetted into the crystallization trays. A number of conditions for crystallization were tested varying the composition of reservoir solution, special additives, ratio of the 70S IC components and conditions of 70S IC formation and preparation. So far the growth of microcrystalline precipitates or spherulites was observed in certain conditions, but no formation of three dimensional crystals. It is possible that the 70S IC preparation used for crystallization was not homogenous enough and contained a considerable portion of the vacant ribosomes and unbound IF2 which prevented three dimensional crystal formation. To increase IF2 incorporation into the 70S IC, we tried to assemble the 70S IC in the presence of all three initiation factors. For this purpose, IF1 and IF3 form $T$. thermophilus were expressed in E. coli and purified.

\section{IF1 activity test.}

IF1 from $T$. thermophilus was tested for activity using the components of the initiation system from $E$. coli as the activities of $E$. coli components were determined previously. Since both systems are homologous, the components from $T$. thermophilus could function in the $E$. coli initiation complex. The activity of IF1 was assessed by measuring the efficiency of $\left.{ }^{3} \mathrm{H}\right] \mathrm{fMet}-\mathrm{tRNA}{ }^{\mathrm{fMet}}$ incorporation into the $30 \mathrm{~S}$ IC by nitrocellulose filter binding. IF1 stimulates 30 S IC formation via promoting IF2 binding to the complex which, in turn, stabilizes fMet-tRNA ${ }^{\mathrm{fMet}}$ on the ribosome (Canonaco et al., 1986; Caserta et al., 2006; Milon et al., 2010). The components of the initiation complexes were mixed together and incubated at $37^{\circ} \mathrm{C}$ for $2 \mathrm{~min}$. Such short-time incubation time was chosen in this case as the kinetics of fMet-tRNA ${ }^{\text {fMet }}$ binding to the $30 \mathrm{~S} \mathrm{IC}$ is different in the presence or absence of initiation factors. The differences of $\mathrm{fMet-tRNA}{ }^{\text {fMet }}$ binding to the ribosome due to IF1 are more prominent when 30S IC formation is performed in a short time. Incubation of the $30 \mathrm{~S} \mathrm{IC} \mathrm{for} \mathrm{time} \mathrm{periods} \mathrm{of} \mathrm{about} 30$ min would mask the differences in binding of $\mathrm{fMet}-\mathrm{tRNA} \mathrm{fMet}^{\mathrm{f}}$ to the $30 \mathrm{~S}$ subunit in the presence or absence of IF1.

The effect of IF1 from T. thermophilus on 30S IC formation is shown on Fig. 4.10. IF1 from $T$. thermophilus functions in the $E$. coli system promoting binding of fMet-tRNA ${ }^{\text {fMet }}$ to the 30 S IC to the same level as IF1 from E. coli. In the absence of IF1, the binding of 
fMet-tRNA ${ }^{\mathrm{fMet}}$ to the $30 \mathrm{~S}$ is approximately 2 times lower after 2 min of incubation time. When no IF1 and IF3 were present in the system, only about $1 \%$ of 30 S subunits were occupied by fMet-tRNA ${ }^{\text {fMet }}$.

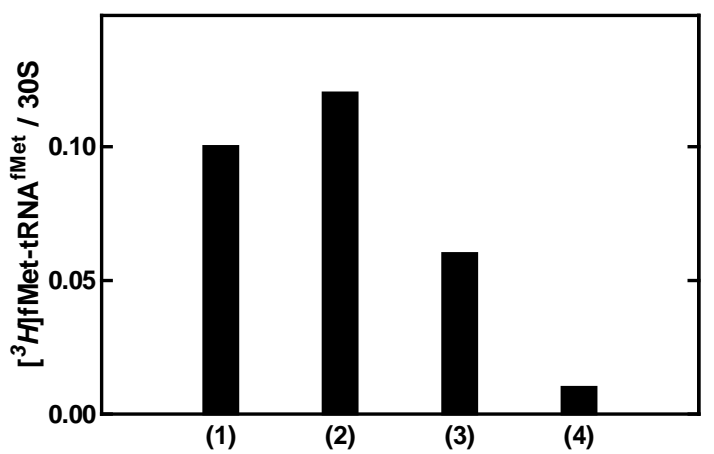

Figure 4.10 30S IC formation (all components from E. coli: $0.4 \mu \mathrm{M}$ 30S, $0.8 \mu \mathrm{M}$ IF2, $0.8 \mu \mathrm{M}$ IF3 or none, $0.8 \mu \mathrm{M}$ fMet-tRNA ${ }^{\text {fMet }}, 1.2 \mu \mathrm{M}$ mRNA, $10 \mu \mathrm{M}$ GTP) using $0.8 \mu \mathrm{M}$ IF1 from $E$. coli (bar (1)), $0.8 \mu \mathrm{M}$ IF1 from $T$. thermophilus (bar (2)), no IF1 (bar (3)), no IF1 and IF3 (bar (4).

\section{IF2 activity test.}

IF2 from $T$. thermophilus was tested for the GTPase activity using a fluorescent GTPase assay. Multiple turnover GTP hydrolysis by IF2 was measured in the time range from $20 \mathrm{~s}$ to 5 min (Fig. 4.11).The GTPase reaction in the T. thermophilus system was measured at $55^{\circ} \mathrm{C}$. For comparison of the overall GTP hydrolysis level in T. thermophilus and E. coli system, the GTPase reaction in the $E$. coli system was measured at $37^{\circ} \mathrm{C}$. We also checked if IF2 from $T$. thermophilus is able to function in the $E$. coli system. In this case, the reaction was carried out at $37^{\circ} \mathrm{C}$. We have seen that IF2 from $T$. thermophilus is active as it hydrolyzes GTP at a level similar to the one of IF2 GTP hydrolysis in the $E$. coli system, although the conditions for the GTPase reaction in $T$. thermophilus system used may be suboptimal. Curiously, we also observed that IF2 from T. thermophilus is capable of GTP hydrolysis in the $E$. coli system, although IF2 from thermophilic organisms is known to have shorter NTD than IF2 from E. coli which may be involved in some important interactions.

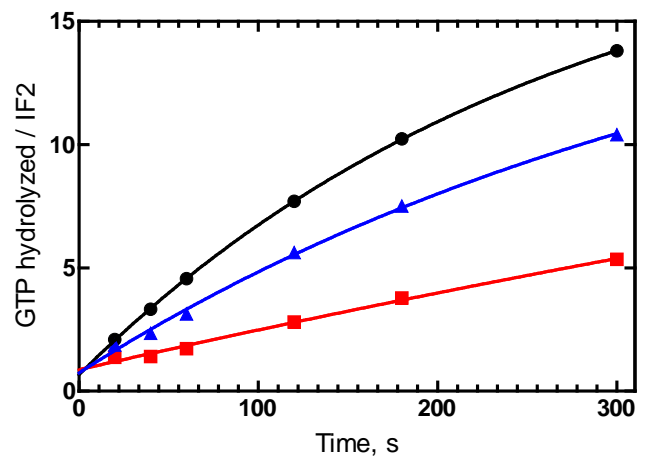

100
Figure 4.11 Comparison of GTPase reaction of IF2 in the E. coli and T. thermophilus systems: black - E.coli system; blue - T. thermophilus system; red - IF2 from $T$. thermophilus in E. coli system. The 70S IC was formed using the following concentrations of the components: $1 \mu \mathrm{M}$ 70S, $2 \mu \mathrm{M}$ IF1, $0.5 \mu \mathrm{M}$ IF2, $2 \mu \mathrm{M}$ IF3, $2 \mu \mathrm{M}$ fMettRNA ${ }^{\text {fMet }}, 3 \mu \mathrm{M}$ mRNA, $10 \mu \mathrm{M}$ mant-GTP. 


\section{IF3 activity test.}

One of the known functions of IF3 is stimulating $70 \mathrm{~S}$ dissociation into the $30 \mathrm{~S}$ and $50 \mathrm{~S}$ subunits (Karimi et al., 1999; Wolfrum et al., 2003). To probe the activity of IF3 from $T$. thermophilus, thermophilic 70S ribosomes were incubated with the factor in the 1:1 or 1:5 ratio respectively. After the incubation time, 70S was separated from $50 \mathrm{~S}$ and $30 \mathrm{~S}$ on sucrose gradients. The absorption profiles of the ribosomes after the separation on sucrose gradients are shown on the Fig 4.10. Without IF3, only one absorption peak is observed which corresponds to intact 705 ribosomes. After the incubation of 705 with IF3 in the 1:1 ration, three absorption peaks are observed. The peaks become more prominent at a 1:5 ratio of ribosomes to IF3 meaning more efficient dissociation of the ribosomes into the subunits (Fig. 4.12).

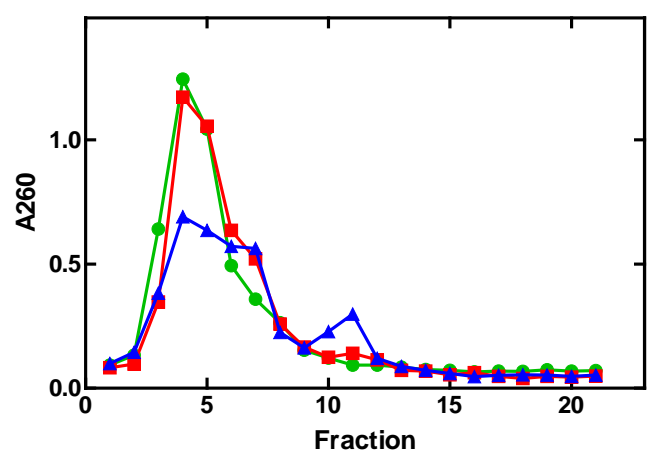

Figure 4.12 Absorption profiles of sucrose gradient after the centrifugation. Green - intact $70 \mathrm{~S}$ in the absence of IF3; red - 70S incubated with IF3 in the ration of $1: 1$; blue - 70 S incubated with IF3 in the ration of 1:5 respectively. Three peaks can be detected: 705 - with the maximum absorption at fraction $4 ; 505$ - with the maximum absorption at fraction 6; 30S with the maximum absorption at a fraction 11.

\section{Reconstitution of 70S IC of T. thermophilus.}

Initiation factors from $T$. thermophilus were first verified using the initiation system components from $E$. coli. Subsequently, we reconstituted the initiation system using the ribosome and all three initiation factors from $T$. thermophilus. The $70 \mathrm{~S}$ initiation complex was formed at varying concentrations of IF2 and incubated at $37^{\circ} \mathrm{C}$ for $30 \mathrm{~min}$. The initiation efficiency was checked by nitrocellulose filter binding (Fig 4.13). The incorporation of fMet-tRNA ${ }^{\text {fMet }}$ into the 70S IC showed no significant dependence on the concentration of IF2 or even IF2 presence. However, the level of incorporation of fMettRNA $^{\text {fMet }}$ into the 70S IC after 30 min of incubation was dependent on the presence of IF1 and IF3. In the presence of all IFs, the incorporation of fMet-tRNA ${ }^{\text {fMet }}$ was 1.4 times higher than in the absence of IF1 and IF3. The explanation is as follows. The canonical initiation pathway starts with the assembly of the initiation complex on the 30 s subunit 
with the $50 \mathrm{~S}$ arriving after the fMet-tRNA ${ }^{\text {fMet }}$ and mRNA are bound to the small ribosomal subunit. In our experiment we started not with the separate ribosomal subunits, but with the 70S ribosome. First, the $70 S$ needed to be separated into the subunits by the action of IF3 and IF1 so that fMet-tRNA ${ }^{\text {fMet }}$ and mRNA could bind. This may explain why we observe increased $f M e t-t R N A^{f M e t}$ incorporation in the presence of IF1 and IF3 compared to their absence. The incorporation of the fMet-tRNA ${ }^{\text {fMet }}$ into the 70 IC did not depend on IF 2 concentration as $30 \mathrm{~min}$ of incubation time was sufficient for the large portion of the fMet-tRNA ${ }^{\text {fMet }}$ to bind the 70S without requiring IF2 function. One should expect to see more significant differences in the fMet-tRNA ${ }^{\text {fMet }}$ binding to the $70 \mathrm{~S}$ after shorter incubation times. Based on this experiment, one can also conclude that the components of the 70S IC from $T$. thermophilus are active as the efficiency of 70 IC formation with all three initiation factors was close to $100 \%$.

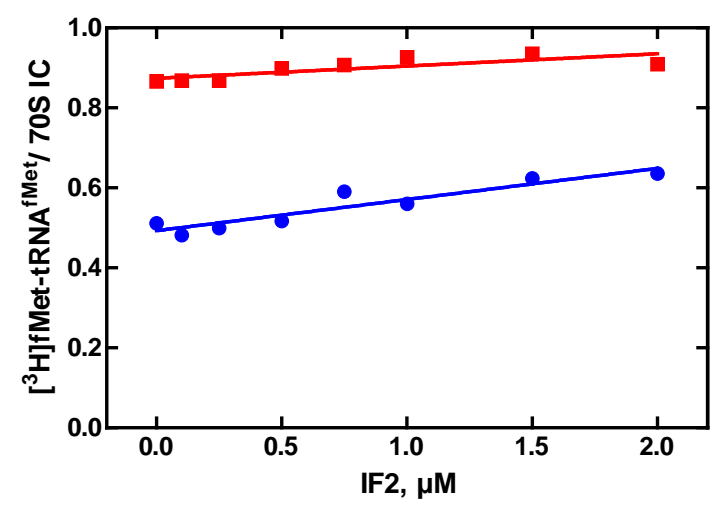

Figure 4.13 70S IC formation measured by the nitrocellulose filter binding. 70S IC was formed in the presence of IF1 and IF3 (red) or in the absence of IF1 and IF3 (blue) with varying IF2 concentrations. 70S IC contained where indicated $0.5 \mu \mathrm{M}$ 70S, $1 \mu \mathrm{M}$ IF1 or none, $1 \mu \mathrm{M}$ IF3 or none, $0.25 \mu \mathrm{M}\left[{ }^{3} \mathrm{H}\right] \mathrm{fMet}-\mathrm{tRNA}{ }^{\mathrm{fMet}}, 1.5 \mu \mathrm{M}$ mRNA, $10 \mu \mathrm{M}$ GDPNP and IF2 in varying concentrations (from $0 \mu \mathrm{M}$ to $2 \mu \mathrm{M}$ ).

\section{Quantification of efficiency of the IF2 binding to the 70S IC.}

The 7OS IC was formed using all three initiation factors, fMet-tRNA ${ }^{\mathrm{fMet}}$, mRNA and a nonhydrolysable GTP analog GDPNP. When GTP hydrolysis is not possible, IF2 does not dissociate from the 70S thus yielding the 70S IC containing not only fMet-tRNA ${ }^{\text {fMet }}$ and mRNA, but also IF2. To quantify the amount of these components bound to the ribosome, analytical gel filtration was performed. It was possible to separate the 70S IC from the excess of the free factors, fMet-tRNA ${ }^{\text {fMet }}$, mRNA and GDPNP and quantify the amount of each component bound to the ribosome. It was not possible, however, to separate vacant 70 S from the 70 S IC using a gel filtration column. 
The 70S IC was reconstituted from the $T$. thermophilus components. To be able to quantify the amount of components bound to the 70S, the following fluorescent derivatives were used: IF2-Atto488, mRNA-Atto488, GDPNP-mant. As IF2 and mRNA were labeled by the same fluorescent label, either of the fluorescent derivatives was used for the reaction, but not both fluorescent derivatives together. We also used radioactive $\left[{ }^{3} \mathrm{H}\right] \mathrm{fMet}-\mathrm{tRNA}{ }^{\mathrm{fMet}}$ for the complex formation. The 70S IC was formed by incubation of the complex at $55^{\circ} \mathrm{C}$ for $30 \mathrm{~min}$. After incubation, the complex was loaded into a gel filtration column (Fig 4.14). As a control, the reaction containing all of the components except the 70S was prepared the same way as the reaction with $70 \mathrm{~S}$ and loaded into the gel filtration column. The elution profile of the ribosome was checked by the absorbance at $260 \mathrm{~nm}$. The elution profile of IF2, mRNA and GDPNP was detected by the fluorescent flow-through detector at the appropriate wave length. The gel filtration fractions were collected and the radioactivity in the fractions was measured, so the amount of the initiator tRNA bound to the 70S IC was determined by radioactive counting. Using the components of the complex labeled with different kinds of fluorescent or radioactive labels, it is possible to simultaneously detect and quantify the amount of every component bound to the ribosome.
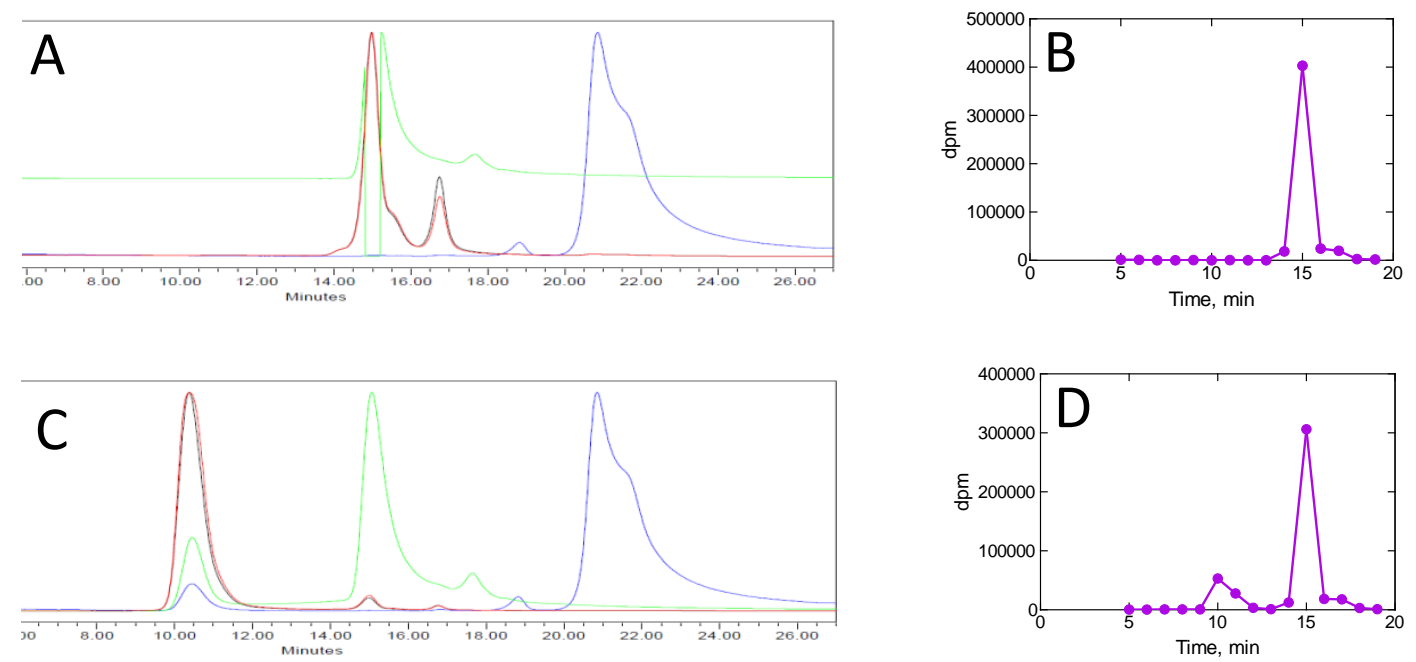

Figure 4.14 The gel filtration of the 70S IC $(1 \mu \mathrm{M}$ 70S, $2 \mu \mathrm{M}$ IF1, $2 \mu \mathrm{M}$ IF2-Atto488, $2 \mu \mathrm{M}$ IF3, $3 \mu \mathrm{M}\left[{ }^{3} \mathrm{H}\right] \mathrm{fMet}-\mathrm{tRNA}{ }^{\mathrm{fMet}}, 3 \mu \mathrm{M}$ mRNA, $20 \mu \mathrm{M}$ mant-GDPNP). The $70 \mathrm{~S}$ IC was formed at $55^{\circ} \mathrm{C}$ for 30 min. A, B - elution profile of the components of the 70S IC in the absence of the 70S ribosome; C, D - elution profile of the 70S IC. Separation of the 70S IC from the excess of free components is observed. A, C - overlaid chromatogram recorded by the absorbance and fluorescent flow-through detectors, where: red line $-70 \mathrm{~S}$ detected by absorbance at $260 \mathrm{~nm}$; green line - IF2-Atto488 detected by fluorescence (ex501 nm/em $523 \mathrm{~nm}$ ); blue line - mantGDPNP detected by fluorescence (ex $360 \mathrm{~nm} / \mathrm{em} 448 \mathrm{~nm}$ ). B, D - elution profile of [ $\left.{ }^{3} \mathrm{H}\right] \mathrm{fMet}-$ tRNA $^{\text {fMet }}$ detected by the radioactive counting of the gel filtration fractions. 
When the 70S was present in the reaction, the coelution of the IF2, fMet-tRNA ${ }^{\text {fMet }}$ and GDPNP was observed together with the $70 \mathrm{~S}$ at the elution time of about $10.5 \mathrm{~min}$ (Fig 4.14, $C$ and D). In the absence of the 70S, free IF2 and fMet-tRNA ${ }^{\text {fMet }}$ were eluted at about $15 \mathrm{~min}$, and free GDPNP at about $21 \mathrm{~min}$ (Fig. 4.14, A and B). The area of the peaks was integrated by the Empower Pro Software (Waters) and the ratio between the bound and total amount of the component was calculated. Knowing the amount of the components initially present in the reaction, the binding efficiency of incorporation of the component to the 70S IC was calculated.

The presence of the fluorescent label on the protein can alter the affinity of the protein to its binding partners. To avoid IF2 labeling with the fluorescent dye, we tried determining its presence in the 70S IC indirectly through measuring the presence of other labeled components. For this purpose $\left[{ }^{3} \mathrm{H}\right] \mathrm{fMet}-\mathrm{tRNA}{ }^{\mathrm{fMet}}$ and mRNA-Atto488 were used as introducing the label does not affect the affinity in this cases (Fig 4.15). However, the amount of $\left[{ }^{3} \mathrm{H}\right] \mathrm{FMet}-\mathrm{tRNA}{ }^{\text {fMet }}$ and mRNA-Atto488 bound to the ribosome after the incubation time of $30 \mathrm{~min}$ in the absence and in the presence of IF2 was the same. This means that neither $\left[{ }^{3} \mathrm{H}\right] \mathrm{fMet}-\mathrm{tRNA}{ }^{\mathrm{fMet}}$ nor mRNA-Atto488 can be used as a marker of IF2 presence in the 70S IC.
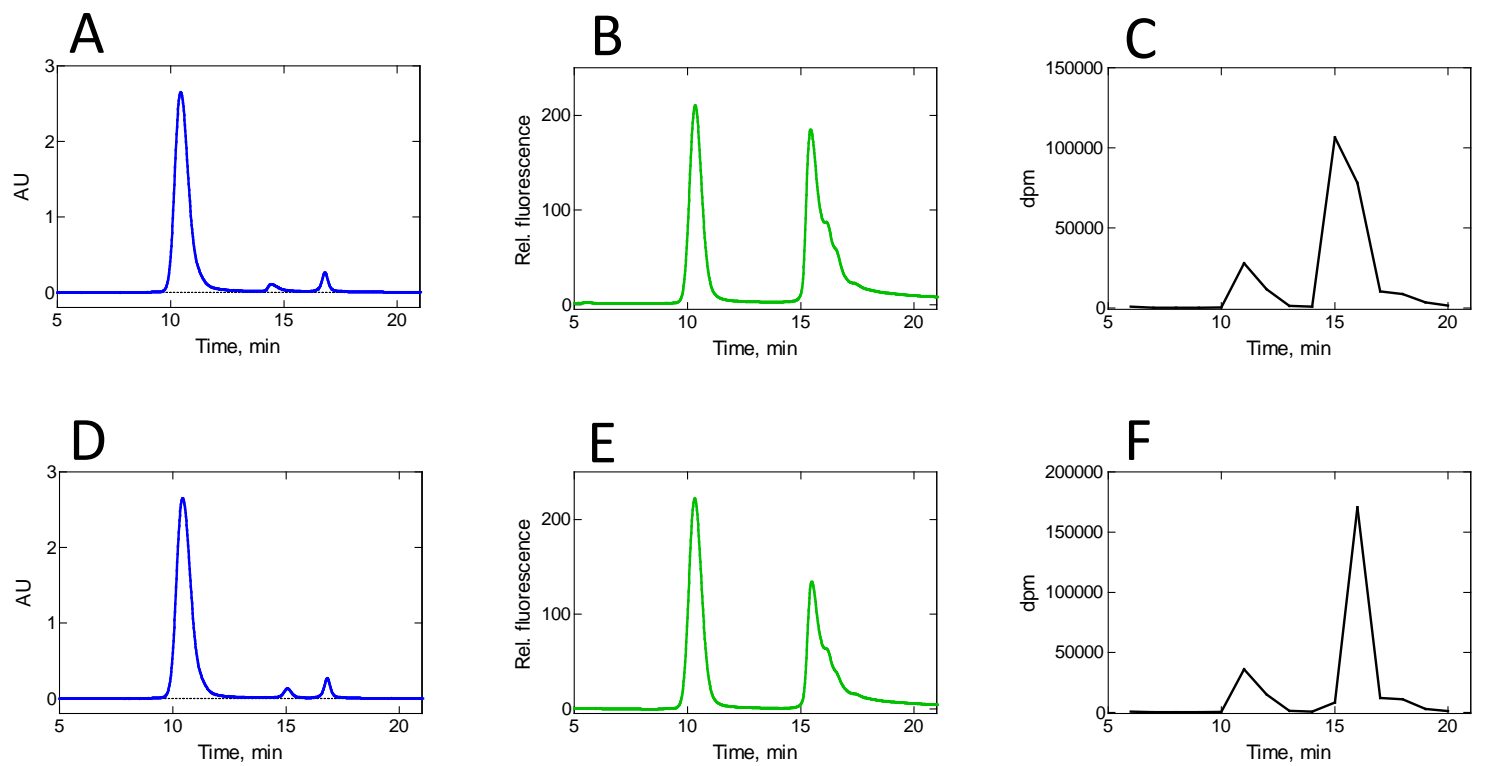

Figure 4.15 (A), (B), (C) The gel filtration of the 70S IC (1 $\mu \mathrm{M}$ 70S, $2 \mu \mathrm{M}$ IF1, $2 \mu \mathrm{M}$ IF2, $2 \mu \mathrm{M}$ IF3, $3 \mu \mathrm{M}\left[{ }^{3} \mathrm{H}\right] \mathrm{fMet}-\mathrm{TRNA}{ }^{\mathrm{fMet}}$, $3 \mu \mathrm{M}$ mRNA-Atto488, $20 \mu \mathrm{M}$ GDPNP). (D), (E), (F) The same, but $70 \mathrm{~S} \mathrm{IC} \mathrm{was} \mathrm{formed} \mathrm{in} \mathrm{the} \mathrm{absence} \mathrm{of} \mathrm{IF2.} \mathrm{The} 70 \mathrm{~S} \mathrm{IC} \mathrm{was} \mathrm{formed} \mathrm{at} 55^{\circ} \mathrm{C}$ for $30 \mathrm{~min}$. Elution profiles: (A), (D) - 70S measured by absorbance at $260 \mathrm{~nm}$; (B), (E) mRNA-Atto488 measured by fluorescence (ex $440 \mathrm{~nm} / \mathrm{em} 523 \mathrm{~nm}$ ); (C),(F) [ $\left.{ }^{3} \mathrm{H}\right] \mathrm{fMet}-\mathrm{tRNA}{ }^{\mathrm{fMet}}$ measured by radioactive counting. 
We also tried detecting IF2 presence in the 70S IC by a different approach. IF2 binds guanine nucleotides. IF2 was incubated with mant-GDPNP and purified from the excess of the fluorescent nucleotide using the desalting column prior to 70S IC formation. We intended to detect IF2 in the 70S IC by the fluorescence of mant-GDPNP and thus avoid labeling of IF2 itself. However it seems not possible to purify IF2 in complex with GDPNP by this method due to the little stability of IF2-GDPNP complex (Fig 4.16).
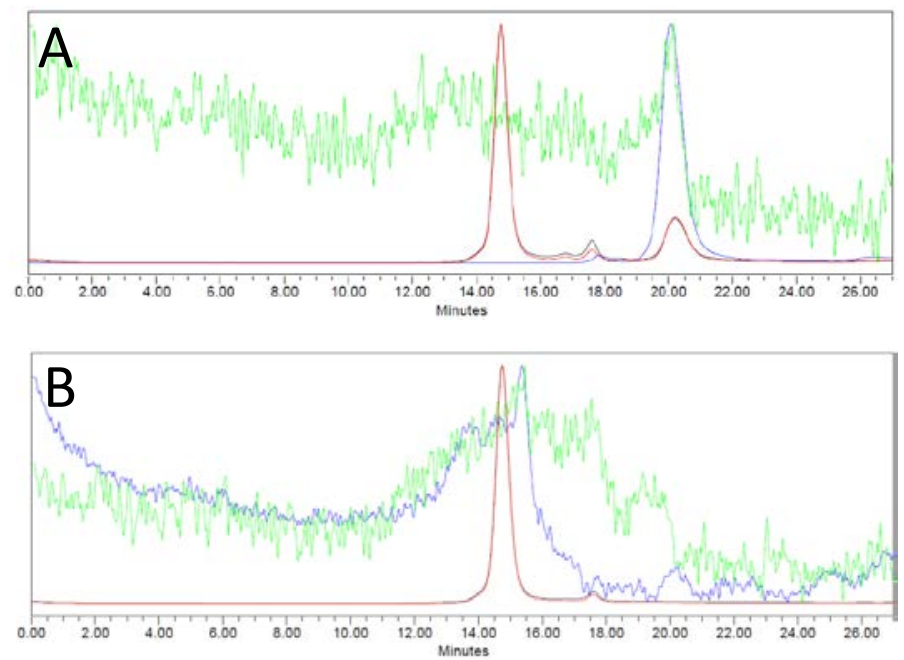

Figure 4.16 Gel filtration of the IF2-GDPNP complex (A) before purification of the complex on the desalting column, (B) after the purification on the desalting column. IF2 is detected by the absorbance at $280 \mathrm{~nm}$ (red), GDPNP is detected by fluorescence (ex $360 \mathrm{~nm} / \mathrm{em} 448 \mathrm{~nm}$ ) (blue). The peak eluting at $14.5 \mathrm{~min}$ corresponds to the IF2; the peak eluting at $20 \mathrm{~min}$ corresponds to the free GDPNP. No nucleotide coelution with IF2 is observed (A). After the purification of the IF2 - GDPNP complex on the desalting column no nucleotide remains in the reaction (B).

We have seen that the presence of IF2 on the $70 \mathrm{~S}$ ribosome is not reflected by the presence of the other components like fMet-tRNA ${ }^{\text {fMet }}$, mRNA of GDPNP. Therefore, to quantify the amount of IF2 bound to the 70S, IF2 had to be detected directly. This can be achieved by fluorescent labeling of IF2. We tried IF2 labeling with $N$-Hydroxysuccinimide (NHS) esters employing two different fluorescent dyes, Atto488 and mant. The NHSesters of the fluorescent dyes react with the amino groups at random positions on the surface of the protein. 70S IC was formed using either IF2-Atto488 or IF2-mant. The 70S IC formation was measured using gel filtration. A portion of the fluorescently labeled IF2 was coeluting with the ribosome meaning it was bound to the 70S IC (Fig. 4.17). The area of the fluorescent peaks was integrated by the Empower Pro Software (Waters) and the amount of the 70S IC formed was calculated. However, according to our calculations, 
the efficiency of IF2 binding to the 70 S was $170 \%$ in this case. As it is not possible that stoichiometry of the IF2 to 70S binding is more than 1, the only possible explanation is that the relative fluorescence level of mant-IF2 is increased upon binding to the 70S IC. If this is the case, mant-IF2 cannot be used for the determination of the efficiency of the IF2 binding to the 70S. An alternative explanation could be that mant-IF2 preparations still contained traces of free mant dye, which could bind the ribosome while incubation of the 7OS IC and hence increase the fluorescence of the entire complex.

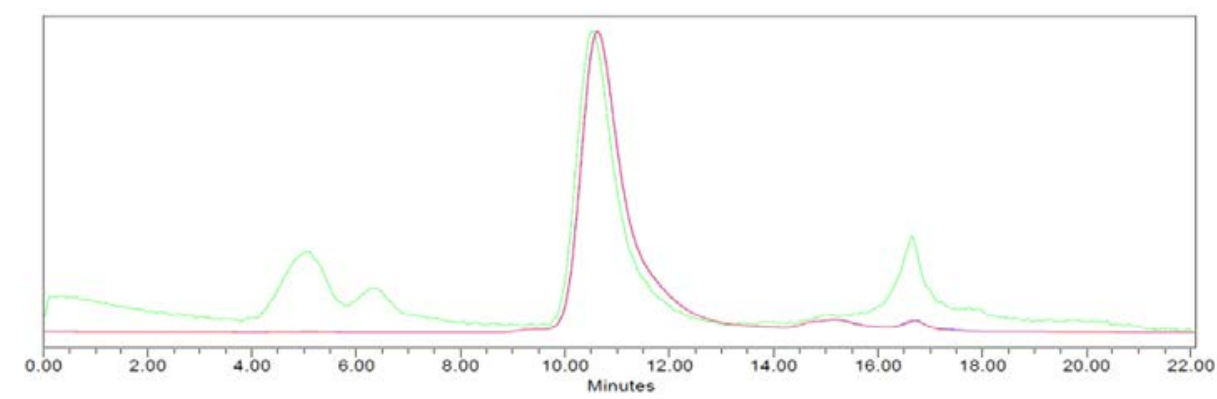

Figure 4.17 Gel filtration of the70S IC formed with mant-IF2 (0.5 $\mu \mathrm{M}$ 70S, $1 \mu \mathrm{M}$ IF1, $1 \mu \mathrm{M}$ IF2, $1 \mu \mathrm{M}$ IF3, $1 \mu \mathrm{M}$ fMet-tRNA ${ }^{\mathrm{fMet}}, 1.5 \mu \mathrm{M}$ mRNA, $10 \mu \mathrm{m}$ GDPNP). Red - elution profile of the ribosome measured by the absorbance at $260 \mathrm{~nm}$; green - fluorescent profile of mantIF2. A peak with maximum intensity at 11 min corresponds to the 70S. A portion of IF2 is coeluting with the ribosome, a peak eluting at 16.5 min corresponds to the free mant-IF2; the fluorescent peaks with the maximum intensities at $5 \mathrm{~min}$ and $6.5 \mathrm{~min}$ may correspond to the aggregates of free fluorescent dye in the preparation.

We also tried 70S IC formation with IF2-Atto488. The coelution of the fluorescent IF2 with $70 \mathrm{~S}$ was observed, suggesting that a portion of IF2 was bound to the ribosome (Fig 4.18, A). However, the precise quantification of the efficiency of the IF2 binding to the 705 was not possible as we found out that IF2-Atto488 preparation was not homogenous, but rather consisted of two populations of IF2 with different level of fluorescent labeling (Fig. 4.18, B). The affinity of these two IF2-Atto488 populations to the 7OS IC as well as the relative fluorescent level could be different and therefore 70S IC formation cannot be measured using such a preparation. 

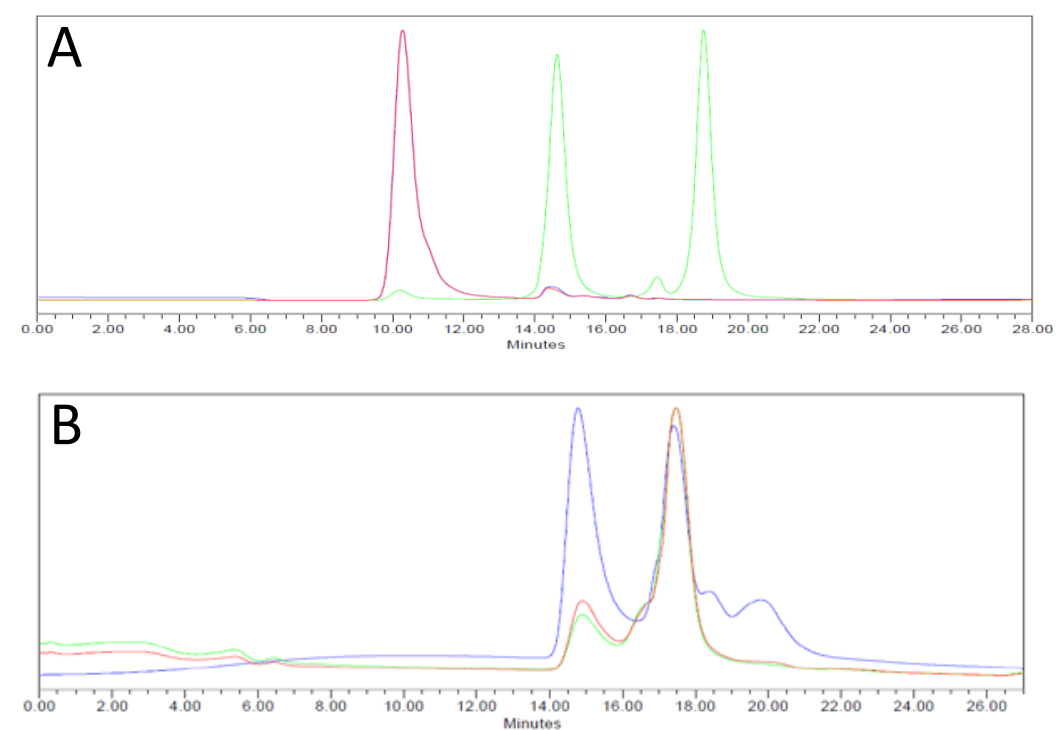

Figure 4.18 (A) Gel filtration of the 70S IC formed with IF2-Atto488 (1 $\mu$ M 70S, $2 \mu \mathrm{M}$ IF1, 4 $\mu \mathrm{M}$ IF2, $2 \mu \mathrm{M}$ IF3, $3 \mu \mathrm{M}$ fMat-tRNA ${ }^{\mathrm{fMet}}, 3 \mu \mathrm{M}$ mRNA, $20 \mu \mathrm{M}$ GDPNP). Red - elution profile of the ribosome measured by the absorbance at $260 \mathrm{~nm}$; green - fluorescent profile of IF2-Atto488. A peak with maximum intensity at 10.5 min corresponds to the $70 \mathrm{~S}$. A small portion of IF2 is coeluting with the ribosome, peaks eluting at $14.5 \mathrm{~min}$ and 17 min may correspond to the populations of IF2 with different degree of Atto488 labeling. (B) Gel filtration of the IF2-Atto488. Red and green - absorbance measured at 260 and $280 \mathrm{~nm}$ respectively; blue - the fluorescence of the IF2-Atto488. Two prominent peaks may correspond to the populations of IF2 with different degree of labeling with Atto488.

IF2-Atto488 was checked for the GTPase activity (Fig. 4.19) to see if the labeled protein maintains the same activity level as the intact IF2. The level of GTP hydrolyzed by IF2Atto488 was found to be $28 \%$ lower than the level of GTP hydrolyzed by intact IF2 after 5 min of reaction. Most probably upon labeling, IF2 GTPase activity is partially lost due to the steric hindrance caused by the fluorophore attached in the vicinity of the GTPase active center or at a binding surface. If this is the case, it is not possible to use IF2Atto488 for quantification of the IF2 incorporated into the 70S IC.

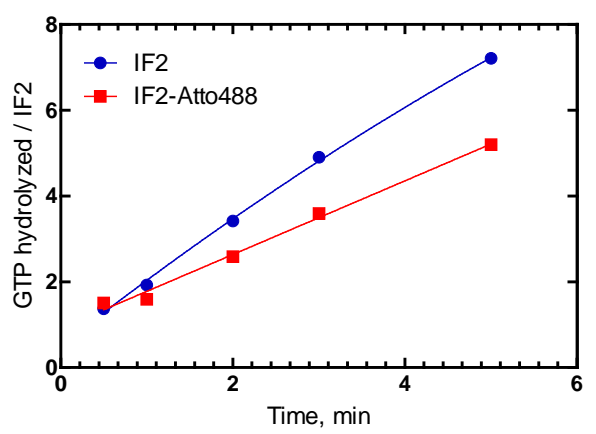

Figure 4.19 GTPase reaction by IF2 (blue) or IF2-Atto488 (red).The loss of GTPase activity by IF2-Atto488 is observed. After 5 min of incubation time the difference in the level of GTP hydrolyzed is $28 \%$. The reactions contained: $0.5 \mu \mathrm{M}$ 70S, $1 \mu \mathrm{M}$ IF1, $0.25 \mu \mathrm{M}$ IF2 or IF2Atto488, $1 \mu \mathrm{M}$ IF3, $1 \mu \mathrm{M}$ fMet-tRNA ${ }^{\text {fMet }}, 1.5 \mu \mathrm{M}$ mRNA, 5 $\mu \mathrm{M}$ mant-GTP. 
To quantify the amount of IF2 bound to the 70S IC, we labeled IF2 with two fluorescent dyes. After complex formation, 70S IC was separated from the unbound components using gel filtration. The portion of IF2 bound to the ribosome was detected by fluorescence of the label attached to IF2. This approach was promising in terms of speed and precision of detection of IF2 bound to the 70S IC. However, it required a long time to establish a reasonable protocol for IF2 labeling and to obtain active homogenous preparation. As the general goal was not IF2 labeling, but quantification of IF2 bound to the 70 S IC, another approach was tested which did not require IF2 modification.

\section{Quantification of IF2 bound to the 70S IC using puromycin reaction.}

IF2 in the GTP-bound form is known to form a contact with the CCA-end of the fMettRNA $^{\text {fMet }}$ (Allen et al., 2005; Myasnikov et al., 2005). It has also been observed on the cryo-EM structures that being in the GDP-bound form, the CTD of IF2 moves away from the initiator tRNA (Allen et al., 2005; Myasnikov et al., 2005). Puromycin is an antibiotic that resembles the $3^{\prime}$ end of the aminoacylated tRNA. It can enter the ribosomal A site and form a peptide bond with the amino acid in the $\mathrm{P}$ site thereby causing premature chain release (Pestka, 1971). Taking the information from the cryo-EM structures into consideration, one can suggest that if the fMet-tRNA ${ }^{\text {fMet }}$ in the P site is shielded by GTPbound IF2, the reaction with puromycin should not occur. In the opposite scenario, if IF2 is not bound to the 70S IC, the reaction with puromycin can take place. Therefore, the reaction with puromycin could be an indicator of IF2 presence in the 70S IC. To mimic the GTP-bound state of IF2, a non-hydrolysable GTP analog, GDPNP, was used in the experiments.

The 70S IC was formed using GDPNP to block IF2 on the 70S IC. For control, 70S IC was formed using GTP. In another control reaction IF2 was omitted from the complex. As expected, no fMet-puromycin formation was seen when 70S IC was formed with GDPNP, as IF2 remained bound to the 70S IC blocking the fMet-group on the TRNA ${ }^{\mathrm{fMet}}$ in the ribosomal P site (Fig 4.20, A, blue). When 70S IC was formed in the presence of GTP, the efficiency of the fMet-puromicin complex formation was close to $100 \%$ (Fig 4.20, A, green). IF2 performed GTP hydrolysis and dissociated from the complex thus making the formylmethionine available for the reaction with the puromycin. In the third reaction IF2 was omitted from the complex (Fig 4.20, A, red) and almost no formation of methioninepuromycin complex was observed, but the amount slightly increased with time. Possibly this corresponds to the rate of spontaneous fMet-tRNA ${ }^{\mathrm{fMet}}$ accommodation in the P site. 108 
To prove that fMet-tRNA ${ }^{\text {fMet }}$ is bound to the ribosome in the absence of IF2, we performed a filter binding experiment using the complexes formed in the same way. After 10 min of incubation, the amount of $\left[{ }^{3} \mathrm{H}\right] \mathrm{fMet}-\mathrm{tRNA} \mathrm{fMet}^{\mathrm{fm}}$ bound to the ribosome in the presence and in the absence of IF2 reached approximately the same level. Even in the absence of IF2 and the nucleotide, fMet-tRNA ${ }^{\text {fMet }}$ is able to bind the ribosome, but is not able to perform the reaction of transpeptidation confirming that the function of IF2 is required for the proper $f M e t-t R N A^{f M e t}$ positioning in the $P$ site. The similar effect was earlier observed on the initiation system from another thermophilic organism B. stearotermophilus (La Teana et al., 1996).
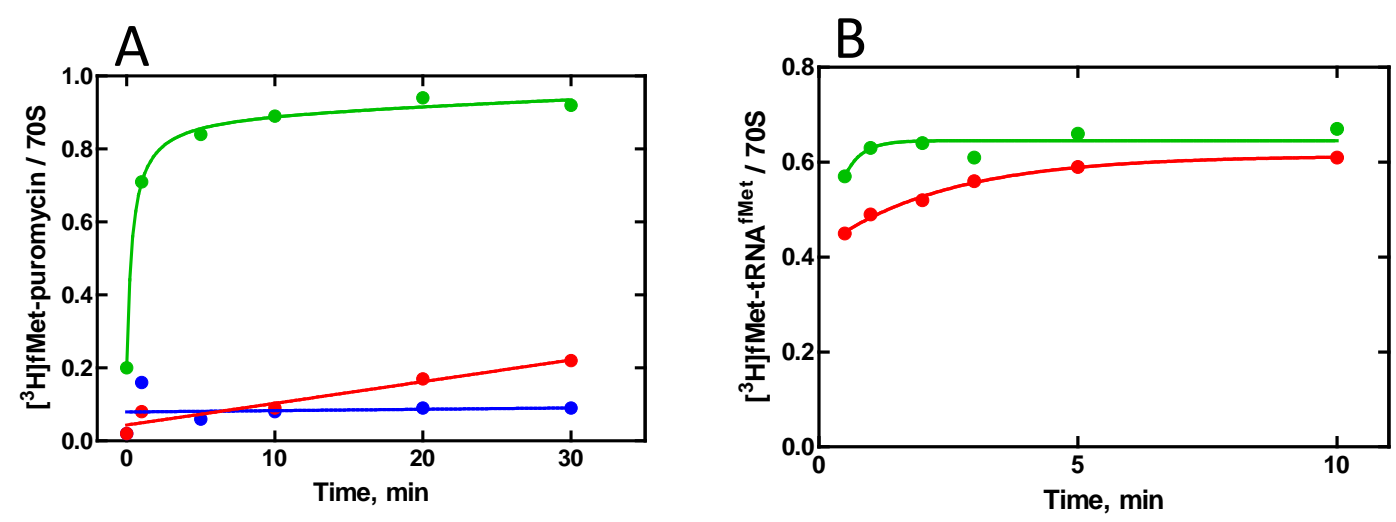

Figure 4.20 (A) Puromycin reaction in the: 70S IC formed with IF2 and GTP (green), 70S IC formed with IF2 and GDPNP (blue), 70S IC formed without IF2 and nucleotide (red). The concentration of the components were as follows: $0.5 \mu \mathrm{M}$ 70S, $1 \mu \mathrm{M}$ IF1, $1 \mu \mathrm{M}$ IF2 or none, $1 \mu \mathrm{M}$ IF3, $0.25 \mu \mathrm{M}$ $\left[{ }^{3} \mathrm{H}\right] \mathrm{fMet}-\mathrm{tRNA}{ }^{\mathrm{fMet}}, 1.5 \mu \mathrm{M}$ mRNA, $10 \mu \mathrm{M}$ GTP or GDPNP or none, $1 \mu \mathrm{M}$ puromycin. (B) Efficiency of $\left[{ }^{3} \mathrm{H}\right] \mathrm{fMet}-\mathrm{tRNA}{ }^{\mathrm{fMet}}$ binding to the $70 \mathrm{~S}$ IC $(0.5 \mu \mathrm{M}$ 70S, $1 \mu \mathrm{M}$ IF1, $1 \mu \mathrm{M}$ IF2 or none, $1 \mu \mathrm{M}$ IF3, $0.25 \mu \mathrm{M}\left[{ }^{3} \mathrm{H}\right.$ ] fMet-tRNA ${ }^{\text {fMet }}, 1.5 \mu \mathrm{M}$ mRNA, $10 \mu \mathrm{M}$ GTP) formed with IF2 (green) or without IF2 (red) measured by nitrocellulose filter binding.

70S IC for crystallization should be formed in the presence of GDPNP to prevent IF2 from dissociating from the ribosome. Therefore we were interested in the efficiency of 70S IC formation using GDPNP nucleotide. Using the puromycin reaction as indicator for the presence of IF2 in the 70S IC, the amount of IF2 bound to the 70S IC was checked (Fig 4.21). At the same time, the amount of fMet-tRNA ${ }^{\text {fMet }}$ bound to the 70S IC in the very same reaction was checked by nitrocellulose filter binding (Fig 4.21). The efficiency of binding of $\mathrm{FMet-tRNA}{ }^{\text {fMet }}$ and IF2 to the ribosome in the presence and in the absence of IF1 and IF3 was also compared. When 70S IC was formed using GDPNP and all three initiation factors, the incorporation of fMet-tRNA ${ }^{\text {fMet }}$ into the complex was 2.5 times 
higher than in the absence of IF1 and IF3. In the presence of all three initiation factors, the efficiency of fMet-puromycin formation is $92 \%$ when GTP is used and $12 \%$ when GDPNP is used, meaning that efficiency of IF2 binding to the ribosome is about $80 \%$.

To have efficient fMet-tRNA ${ }^{\text {fMet }}$ and IF2 incorporation into the 70S IC formed in the presence of GDPNP, the complex should be formed using all three initiation factors. As the 7OS IC is aimed to be used for crystallization, the purification of the complex from IF1 and IF3 as well as the excess of the other components is required.

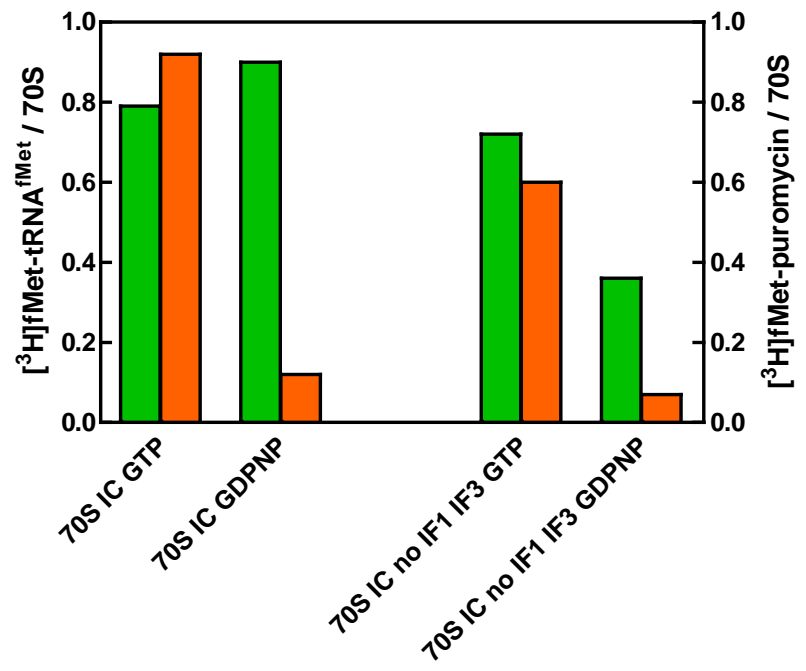

Figure 4.21 The efficiency of fMet-tRNA ${ }^{\text {fMet }}$ (green; checked by nitrocellulose filter binding) and IF2 (orange; checked by puromycin reaction) binding to the 7OS IC in the presence of GTP or GDPNP. The influence of IF1 and IF3 on the incorporation of fMet-tRNA ${ }^{\text {fMet }}$ and IF2 is also checked. The70S IC was formed using the following concentrations of the components: $0.5 \mu \mathrm{M}$ 70S, $1 \mu \mathrm{M}$ IF1 or none, $1 \mu \mathrm{M}$ IF2, $1 \mu \mathrm{M}$ IF3 or none, $0.25 \mu \mathrm{M}$ $\left[{ }^{3} \mathrm{H}\right]$ fMet-tRNA ${ }^{\mathrm{fMet}}, 1.5 \mu \mathrm{M}$ mRNA, $10 \mu \mathrm{M}$ GTP or GDPNP, $1 \mathrm{mM}$ puromycin. The complex was incubated at $37^{\circ} \mathrm{C}$ for $20 \mathrm{~min}$.

\section{IC purification and IF 2 rebinding.}

To get rid of the excess of initiation factors, the 70S IC was formed in the presence of GTP and purified through the sucrose cushion. After purification, 70S IC contains fMettRNA $^{\text {fMet }}$ and mRNA, but no IF2. However, it is possible to rebind IF2 in complex with GDPNP to the purified 70S IC. We incubated 70S IC with increasing concentrations of IF2GDPNP and used puromycin reaction to measure the efficiency of IF2-GDPNP rebinding to the preformed 70S IC. The portion of IF2 bound was dependent on the IF2 
concentration (Fig 4.22). Rebinding of IF2 to the $70 \mathrm{~S} \mathrm{IC} \mathrm{at} 55^{\circ} \mathrm{C}$ was ca. $10 \%$ more effective than rebinding at $37^{\circ} \mathrm{C}$ (Fig 4.22). The maximum level of IF2 incorporation to the 70S IC using this method was $70 \%$ out of the amount of 70S IC formed. The IF2 binding dependence was described by a hyperbolic function. The extrapolation of the dependence showed the concentration of IF2 that should be used to reach an efficiency of $70 \mathrm{~S} \mathrm{IC} \mathrm{formation} \mathrm{close} \mathrm{to} 100 \%$. We consider the suggested protocol for 70S IC preparation suitable for further crystallization experiments.

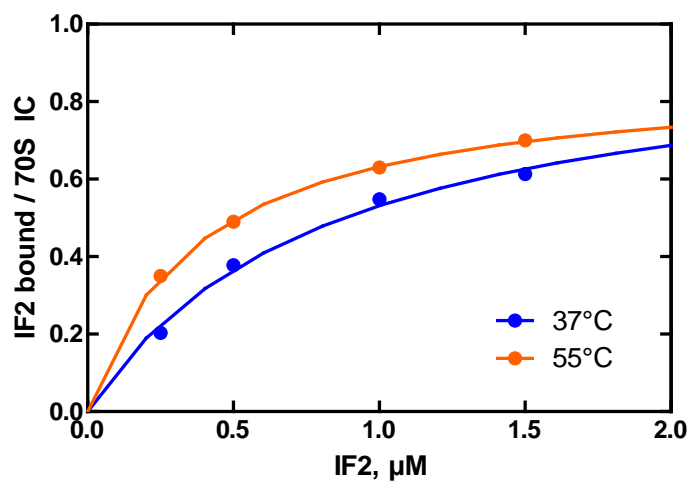

Figure 4.22 IF2 binding to the 70S IC measured by the puromycin reaction. IF2 binding was carried on at $37^{\circ} \mathrm{C}$ (blue) or $55^{\circ} \mathrm{C}$ (orange). The 70 S IC ( $0.25 \mu \mathrm{M}$ 70S, $0.6 \mu \mathrm{M}$ IF1, $0.6 \mu \mathrm{M}$ IF2, $0.6 \mu \mathrm{M}$ IF3, $0.6 \mu \mathrm{M}\left[{ }^{3} \mathrm{H}\right.$ ]fMet-tRNA ${ }^{\text {fMet }}, 0.6 \mu \mathrm{M}$ mRNA, $10 \mu \mathrm{M} \mathrm{GTP}$ ) was prepared at $37^{\circ} \mathrm{C}$ for $30 \mathrm{~min}$ and purified through the sucrose cushion. Various concentration of IF2 (0.25 $\mu \mathrm{M}, 0.5$ $\mu \mathrm{M}, 1 \mu \mathrm{M}, 1.5 \mu \mathrm{M}$ ) were incubated with the purified 70S IC for IF2 rebinding.

\section{Discussion}

The goal of the current project was the crystallization of 70S IC containing IF2 bound to the complex. 70S IC in its final stage contains mRNA and fMet-tRNA ${ }^{f M e t}$ in the $P$ site of the ribosome. In this state the complex is rather stable and ready to enter elongation. However, the aim was to trap the 70S IC at its early stage, right after the 50S subunit has associated to the 30S IC, but prior IF2 dissociating. One of the approaches to stabilize the translational factors on the ribosome for crystallization is to use antibiotics that prevent factors dissociation from the ribosome thereby blocking the translational complexes at particular step. So far no antibiotic is known that allows to block IF2 on the ribosome. As IF2 is a GTPase, we used the non-hydrolysable GTP analog GDPNP for 70S IC formation thus preventing IF2 from dissociation off the complex. 
The components for crystallization were purified from $T$. thermophilus which is a model organism for crystallization studies. It is a Gram-negative thermophilic bacterium which lives in the hot springs at $65^{\circ} \mathrm{C}-80^{\circ} \mathrm{C}$. The components from thermophilic organisms are typically used for crystallization as they are more stable at lower temperatures. A number of ribosomal complexes were successfully crystallized to date using components purified from T. thermophilus (Gao et al., 2009; Jenner et al., 2013; Jin et al., 2011; Schmeing et al., 2009; Voorhees et al., 2010; Yusupov et al., 2001; Yusupova et al., 2001; Zhou et al., 2012)

It was earlier found that in the wild type ribosomes the preferred crystal packing involved protein L9 thus preventing binding of translational GTPases to the ribosome (Selmer et al., 2012). Therefore for our crystallization studies we used the mutant $70 \mathrm{~S}$ ribosomes from $T$. thermophilus HB8 strain which was initially introduced by $V$. Ramakrishnan. The mutant ribosomes lack large ribosomal subunit protein L9 (Selmer et al., 2012). A number of ribosomal complexes with translation GTPases were successfully crystalized using 70S LI9 mutant (Gao et al., 2009; Jin et al., 2011; Schmeing et al., 2009; Voorhees et al., 2010).

The IF2 containing 70S IC for crystallization could be obtained by two different approaches either involving or avoiding the purification of the formed complex. First, we tried the approach where all the components of the 70S IC including the ribosome, fMet-tRNA $^{\text {fMet }}$, mRNA, IF2 and GDPNP were mixed together and crystallized without subsequent 70S IC purification. The vast variety of conditions for crystallization was tested, including the ratio of components, crystallization solutions, additives and the procedure of complex formation. No crystals of the 70S IC was obtained so far using this approach. The possible reasons could be the inefficient complex formation and hence lack of homogeneity in the crystallization sample.

To increase the efficiency of 70S IC formation, we reconstituted the complete translation initiation system from $T$. thermophilus. IF1 and IF3 from $T$. thermophilus were purified and the activity of initiation factors was tested using the T.thermophilus system as well as the heterologous E. coli system. The factors were shown to be active in both systems and conditions for the $T$. thermophilus 70 S IC formation were optimized. Importantly, $T$. thermophilus IF2 could perform GTP hydrolysis on the 70S IC meaning that the preparation of the factor is functionally active and is capable of forming the proper initiation complex. 70S IC was formed by incubating the ribosome with all three initiation factors. $70 \mathrm{~S}$ ribosome had to be dissociated into to the subunits with the help 
of IF3 to allow the efficient binding of initiation factors, fMet-tRNA ${ }^{\text {fMet }}$ and mRNA on the small ribosomal subunit. Subsequently the large ribosomal subunit associated with the $30 \mathrm{~S} \mathrm{IC}$. The presence of IF2 in the 70S IC is important for the proper positioning of the fMet-tRNA $^{\text {fMet }}$ in the $P$ site of the ribosome. As the complex was formed in the presence of GDPNP, IF2 could not perform GTP hydrolysis and remained bound to the ribosome after 505 joining.

Next, the amount of IF2 bound to the 70S IC had to be estimated. Two approaches were possible for detecting the IF2 in the complex - direct and indirect estimation of IF2 bound to the ribosome. The indirect approach required the quantification of other labeled components bound to the 70S IC, like $\left[{ }^{3} \mathrm{H}\right] \mathrm{fMet}-\mathrm{tRNA}{ }^{\mathrm{fMet}}$, mRNA-Atto488 or mant-GDPNP. This approach had the advantage of using intact IF2 for the 70S IC formation. The quantification procedure of all of the 70S IC components using analytical gel filtration was established. However the presence of $\left[{ }^{3} \mathrm{H}\right] \mathrm{fMet}-\mathrm{tRNA}{ }^{\text {fMet }}$, mRNAAtto488 or mant-GDPNP in the 70S IC did not depend quantitatively on the IF2 presence in the complex. Therefore a direct IF2 quantification approach should be used. We used fluorescent labeled IF2 and analytical gel filtration analysis. This approach had advantage of fast and easy direct quantification of the protein bound to the ribosome. At the same time, the fluorescent label could alter the affinity of IF2 to the 70S or the relative fluorescence of the labeled IF2 could be changed upon binding to the ribosome. We tried labeling IF2 with two fluorescent dyes - mant-NHS and Atto488-NHS. However partial GTPase activity loss was found in the fluorescent preparation of IF2. Moreover, two populations of IF2 differing in the degree of labeling were seen when analyzing the fluorescent IF2 preparations by native PAGE or gel filtration. The IF2 labeling procedure required careful establishment and conditions optimization. As obtaining fluorescent derivative of IF2 was not the aim of the current project, we tried yet another approach of determining the amount of IF2 bound to the 70S IC. Cryo-EM reconstructions of the 70S IC with IF2 were previously obtained showing that IF2 bound to GTP formed an interaction with the CCA-end of the fMet-tRNA ${ }^{\mathrm{fMet}}$ (Allen et al., 2005; Julián et al., 2011; Myasnikov et al., 2005). This would prevent formylmethionine on the tRNA ${ }^{\text {fMet }}$ from reacting with puromycin as the CCA-end of the initiator tRNA would be shielded by IF2. The reaction of $\mathrm{fMet}$ with puromycin was used as an indicator reaction for the presence of IF2 in the 70S IC. The final level of the product formed in the reaction had reverse dependence from the amount of IF2 bound. In the absence of IF2, fMet-tRNA ${ }^{\text {fMet }}$ was bound to the ribosome but could not participate in the reaction of transpeptidation. The 
effect is possibly due to the incorrect $f M e t-t R N A^{f M e t}$ orientation in the $P$ site in the absence of IF2.

Including IF1 and IF3 in the 70S IC formation increased the incorporation of fMettRNA ${ }^{\text {fMet }}$ and IF2 into the complex however it introduced the need of a subsequent step of 70 S IC purification for crystallization. After 70S IC formation, the complex was purified from the excess of the initiation factors through the sucrose cushion. This yielded the 70S IC containing fMet-tRNA ${ }^{\text {fMet }}$ and mRNA but no IF2. This step is important as it allows to have most of the ribosomes in the pool (>90\%) occupied with the fMet-tRNA ${ }^{\text {fMet }}$ and mRNA. After sucrose cushion purification, IF2 in complex with GDPNP was bound to the $70 S$ complex again. So far we managed to obtain the efficiency of IF2 rebinding of about $70 \%$. The described procedure of 70 S IC purification will be used for further crystallization experiments. 


\section{References.}

Allen, G.S., Zavialov, A., Gursky, R., Ehrenberg, M., and Frank, J. (2005). The cryo-EM structure of a translation initiation complex from Escherichia coli. Cell 121, 703-712.

Allert, M., Cox, J., and Hellinga, H. (2010). Multifactorial determinants of protein expression in prokaryotic open reading frames. Journal of Molecular Biology 402, 905918.

Antoun, A., Pavlov, M.Y., Andersson, K., Tenson, T., and Ehrenberg, M. (2003). The roles of initiation factor 2 and guanosine triphosphate in initiation of protein synthesis. The EMBO Journal 22, 5593-5601.

Antoun, A., Pavlov, M.Y., Lovmar, M., and Ehrenberg, M. (2006a). How initiation factors tune the rate of initiation of protein synthesis in bacteria. The EMBO Journal 25, 25392550.

Antoun, A., Pavlov, M.Y., Lovmar, M., and Ehrenberg, M. (2006b). How initiation factors maximize the accuracy of tRNA selection in initiation of bacterial protein synthesis. Molecular Cell 23, 183-193.

Bae, W., Xia, B., Inouye, M., and Severinov, K. (2000). Escherichia coli CspA-family RNA chaperones are transcription antiterminators. Proceedings of the National Academy of Sciences of the United States of America 97, 7784-7789.

Bashan, A., and Yonath, A. (2008). Correlating ribosome function with high-resolution structures.

Baudet, M., Ortet, P., Gaillard, J.-C., Fernandez, B., Guérin, P., Enjalbal, C., Subra, G., De Groot, A., Barakat, M., Dedieu, A., et al. (2010). Proteomics-based refinement of Deinococcus deserti genome annotation reveals an unwonted use of non-canonical translation initiation codons. Molecular \& Cellular Proteomics : MCP 9, 415-426.

Bingel-Erlenmeyer, R., Kohler, R., Kramer, G., Sandikci, A., Antolić, S., Maier, T., Schaffitzel, C., Wiedmann, B., Bukau, B., and Ban, N. (2008). A peptide deformylaseribosome complex reveals mechanism of nascent chain processing. Nature $452,108-$ 111. 
Binns, N., and Masters, M. (2002). Expression of the Escherichia coli pcnB gene is translationally limited using an inefficient start codon: a second chromosomal example of translation initiated at AUU. Molecular Microbiology 44, 1287-1298.

Biou, V., Shu, F., and Ramakrishnan, V. (1995). X-ray crystallography shows that translational initiation factor IF3 consists of two compact alpha/beta domains linked by an alpha-helix. The EMBO Journal 14, 4056-4064.

Blattner, F.R., Plunkett, G., Bloch, C.A., Perna, N.T., Burland, V., Riley, M., Collado-Vides, J., Glasner, J.D., Rode, C.K., Mayhew, G.F., et al. (1997). The complete genome sequence of Escherichia coli K-12. Science (New York, N.Y.) 277, 1453-1462.

Boelens, R., and Gualerzi, C.O. (2002). Structure and function of bacterial initiation factors. Current Protein \& Peptide Science 3, 107-119.

Boileau, G., Butler, P., Hershey, J.W., and Traut, R.R. (1983). Direct cross-links between initiation factors 1,2 , and 3 and ribosomal proteins promoted by 2-iminothiolane. Biochemistry 22, 3162-3170.

Bollen, a, Heimark, R.L., Cozzone, a, Traut, R.R., and Hershey, J.W. (1975). Cross-linking of initiation factor IF-2 to Escherichia coli $30 \mathrm{~S}$ ribosomal proteins with dimethylsuberimidate. The Journal of Biological Chemistry 250, 4310-4314.

Boni, I. V, Isaeva, D.M., Musychenko, M.L., and Tzareva, N. V (1991). Ribosomemessenger recognition: mRNA target sites for ribosomal protein S1. Nucleic Acids Research 19, 155-162.

Bonissone, S., Gupta, N., Romine, M., Bradshaw, R.A., and Pevzner, P.A. (2013). Nterminal protein processing: a comparative proteogenomic analysis. Molecular \& Cellular Proteomics : MCP 12, 14-28.

Brandt, R., and Gualerzi, C.O. (1991). Ribosome-mRNA contact sites at different stages of translation initiation as revealed by cross-linking of model mRNAs. Biochimie 73, 15431549.

Brock, S., Szkaradkiewicz, K., and Sprinzl, M. (1998). Initiation factors of protein biosynthesis in bacteria and their structural relationship to elongation and termination factors. Molecular Microbiology 29, 409-417.

Butler, J.S., Springer, M., Dondon, J., Graffe, M., and Grunberg-Manago, M. (1986). Escherichia coli protein synthesis initiation factor IF3 controls its own gene expression at the translational level in vivo. Journal of Molecular Biology 192, 767-780. 
Butler, J.S., Springer, M., and Grunberg-Manago, M. (1987). AUU-to-AUG mutation in the initiator codon of the translation initiation factor IF3 abolishes translational autocontrol of its own gene (infC) in vivo. Proceedings of the National Academy of Sciences of the United States of America 84, 4022-4025.

Caldas, T., Laalami, S., and Richarme, G. (2000). Chaperone properties of bacterial elongation factor EF-G and initiation factor IF2. The Journal of Biological Chemistry 275, 855-860.

Calogero, R.A., Pon, C.L., Canonaco, M.A., and Gualerzi, C.O. (1988). Selection of the mRNA translation initiation region by Escherichia coli ribosomes. Proceedings of the National Academy of Sciences of the United States of America 85, 6427-6431.

Canonaco, M.A., Calogero, R.A., and Gualerzi, C.O. (1986). Mechanism of translational initiation in prokaryotes. Evidence for a direct effect of IF2 on the activity of the $30 \mathrm{~S}$ ribosomal subunit. FEBS Letters 207, 198-204.

Canonaco, M.A., Gualerzi, C.O., and Pon, C.L. (1989). Alternative occupancy of a dual ribosomal binding site by mRNA affected by translation initiation factors. European Journal of Biochemistry / FEBS 182, 501-506.

Carter, a P., Clemons, W.M., Brodersen, D.E., Morgan-Warren, R.J., Hartsch, T., Wimberly, B.T., and Ramakrishnan, V. (2001). Crystal structure of an initiation factor bound to the 30 S ribosomal subunit. Science (New York, N.Y.) 291, 498-501.

Caserta, E., Tomsic, J., Spurio, R., La Teana, A., Pon, C.L., and Gualerzi, C.O. (2006). Translation initiation factor IF2 interacts with the $30 \mathrm{~S}$ ribosomal subunit via two separate binding sites. Journal of Molecular Biology 362, 787-799.

Caserta, E., Ferrara, C., Milon, P., Fabbretti, A., Rocchetti, A., Tomsic, J., Pon, C.L., Gualerzi, C.O., and La Teana, A. (2010). Ribosomal interaction of Bacillus stearothermophilus translation initiation factor IF2: characterization of the active sites. Journal of Molecular Biology 396, 118-129.

Celano, B., Pawlik, R.T., and Gualerzi, C.O. (1988). Interaction of Escherichia coli translation-initiation factor IF-1 with ribosomes. European Journal of Biochemistry / FEBS 178, 351-355.

Chae, Y.B., Mazumder, R., and Ochoa, S. (1969). Polypeptide chain initiation in E. coli: isolation of homogeneous initiation factor E2 and its relation to ribosomal proteins. Proceedings of the National Academy of Sciences of the United States of America 62, 1181-1188. 
Chang, B., Halgamuge, S., and Tang, S.-L. (2006). Analysis of SD sequences in completed microbial genomes: non-SD-led genes are as common as SD-led genes. Gene 373, 90-99.

Chaudhuri, J., Si, K., and Maitra, U. (1997). Function of eukaryotic translation initiation factor $1 \mathrm{~A}$ (elF1A) (formerly called elF-4C) in initiation of protein synthesis. The Journal of Biological Chemistry 272, 7883-7891.

Choi, S.K., Lee, J.H., Zoll, W.L., Merrick, W.C., and Dever, T.E. (1998). Promotion of mettRNAiMet binding to ribosomes by yIF2, a bacterial IF2 homolog in yeast. Science (New York, N.Y.) 280, 1757-1760.

De Cock, E., Springer, M., and Dardel, F. (1999). The interdomain linker of Escherichia coli initiation factor IF3: a possible trigger of translation initiation specificity. Molecular Microbiology 32, 193-202.

Cory, S., Marcker, K.A., Dube, S.K., and Clark, B.F. (1968). Primary structure of a methionine transfer RNA from Escherichia coli. Nature 220, 1039-1040.

Croitoru, V., Bucheli-Witschel, M., Hagg, P., Abdulkarim, F., and Isaksson, L. a. (2004). Generation and characterization of functional mutants in the translation initiation factor IF1 of Escherichia coli. European Journal of Biochemistry 271, 534-544.

Croitoru, V. V, Bucheli-Witschel, M., and Isaksson, L. a (2005). In vivo involvement of mutated initiation factor IF1 in gene expression control at the translational level. FEBS Letters 579, 995-1000.

Cummings, H.S., and Hershey, J.W. (1994). Translation initiation factor IF1 is essential for cell viability in Escherichia coli. Journal of Bacteriology 176, 198-205.

Dahlquist, K.D., and Puglisi, J.D. (2000). Interaction of translation initiation factor IF1 with the E. coli ribosomal A site. Journal of Molecular Biology 299, 1-15.

Dallas, a, and Noller, H.F. (2001). Interaction of translation initiation factor 3 with the 30 S ribosomal subunit. Molecular Cell 8, 855-864.

Debey, P., Hui Bon Hoa, G., Douzou, P., Godefroy-Colburn, T., Graffe, M., and GrunbergManago, M. (1975). Ribosomal subunit interaction as studied by light scattering. Evidence of different classes of ribosome preparations. Biochemistry 14, 1553-1559.

Demeshkina, N., Jenner, L., Westhof, E., Yusupov, M., and Yusupova, G. (2012). A new understanding of the decoding principle on the ribosome. Nature 484, 256-259. 
Dreyfus, M. (1988). What constitutes the signal for the initiation of protein synthesis on Escherichia coli mRNAs? Journal of Molecular Biology 204, 79-94.

Dube, S.K., Marcker, K.A., Clark, B.F., and Cory, S. (1968). Nucleotide sequence of Nformyl-methionyl-transfer RNA. Nature 218, 232-233.

Dubnoff, J.S., and Maitra, U. (1972). Characterization of the ribosome-dependent guanosine triphosphatase activity of polypeptide chain initiation factor IF 2 . The Journal of Biological Chemistry 247, 2876-2883.

Fabbretti, A., Pon, C.L., Hennelly, S.P., Hill, W.E., Lodmell, J.S., and Gualerzi, C.O. (2007). The real-time path of translation factor IF3 onto and off the ribosome. Molecular Cell 25, 285-296.

Fabbretti, A., Brandi, L., Milón, P., Spurio, R., Pon, C.L., and Gualerzi, C.O. (2012). Translation initiation without IF2-dependent GTP hydrolysis. Nucleic Acids Research 40, 7946-7955.

Fakunding, J.L., Traut, R.R., and Hershey, J.W. (1973). Dependence of initiation factor IF2 activity on proteins L7 and L12 from Escherichia coli $50 \mathrm{~S}$ ribosomes. The Journal of Biological Chemistry 248, 8555-8559.

Fortier, P.L., Schmitter, J.M., Garcia, C., and Dardel, F. (1994). The N-terminal half of initiation factor IF3 is folded as a stable independent domain. Biochimie 76, 376-383.

Fourmy, D., Mechulam, Y., Brunie, S., Blanquet, S., and Fayat, G. (1991). Identification of residues involved in the binding of methionine by Escherichia coli methionyl-tRNA synthetase. FEBS Letters 292, 259-263.

Gao, Y.-G., Selmer, M., Dunham, C.M., Weixlbaumer, A., Kelley, A.C., and Ramakrishnan, V. (2009). The structure of the ribosome with elongation factor $G$ trapped in the posttranslocational state. Science (New York, N.Y.) 326, 694-699.

Garcia, C., Fortier, P.L., Blanquet, S., Lallemand, J.Y., and Dardel, F. (1995a). 1H and 15N resonance assignments and structure of the $\mathrm{N}$-terminal domain of Escherichia coli initiation factor 3. European Journal of Biochemistry / FEBS 228, 395-402.

Garcia, C., Fortier, P.L., Blanquet, S., Lallemand, J.Y., and Dardel, F. (1995b). Solution structure of the ribosome-binding domain of $\mathrm{E}$. coli translation initiation factor IF3. Homology with the U1A protein of the eukaryotic spliceosome. Journal of Molecular Biology 254, 247-259. 
Gaur, R., Grasso, D., Datta, P.P., Krishna, P.D. V, Das, G., Spencer, A., Agrawal, R.K., Spremulli, L., and Varshney, U. (2008). A single mammalian mitochondrial translation initiation factor functionally replaces two bacterial factors. Molecular Cell 29, 180-190.

Giuliodori, A.M., Brandi, A., Gualerzi, C.O., and Pon, C.L. (2004). Preferential translation of cold-shock mRNAs during cold adaptation. RNA (New York, N.Y.) 10, 265-276.

Giuliodori, A.M., Brandi, A., Giangrossi, M., Gualerzi, C.O., and Pon, C.L. (2007). Coldstress-induced de novo expression of infC and role of IF3 in cold-shock translational bias. RNA (New York, N.Y.) 13, 1355-1365.

Gold, L. (1988). Posttranscriptional regulatory mechanisms in Escherichia coli. Annual Review of Biochemistry 199-233.

Gribskov, M. (1992). Translational initiation factors IF-1 and elF-2 alpha share an RNAbinding motif with prokaryotic ribosomal protein S1 and polynucleotide phosphorylase. Gene 119, 107-111.

Grigoriadou, C., Marzi, S., Kirillov, S., Gualerzi, C.O., and Cooperman, B.S. (2007a). A quantitative kinetic scheme for $70 \mathrm{~S}$ translation initiation complex formation. Journal of Molecular Biology 373, 562-572.

Grigoriadou, C., Marzi, S., Pan, D., Gualerzi, C.O., and Cooperman, B.S. (2007b). The translational fidelity function of IF3 during transition from the $30 \mathrm{~S}$ initiation complex to the $70 \mathrm{~S}$ initiation complex. Journal of Molecular Biology 373, 551-561.

Grill, S., Gualerzi, C.O., Londei, P., and Bläsi, U. (2000). Selective stimulation of translation of leaderless mRNA by initiation factor 2: evolutionary implications for translation. The EMBO Journal 19, 4101-4110.

Grill, S., Moll, I., Hasenöhrl, D., Gualerzi, C.O., and Bläsi, U. (2001). Modulation of ribosomal recruitment to 5 '-terminal start codons by translation initiation factors IF2 and IF3. FEBS Letters 495, 167-171.

Grunberg-Manago, M., Dessen, P., Pantaloni, D., Godefroy-Colburn, T., Wolfe, a D., and Dondon, J. (1975). Light-scattering studies showing the effect of initiation factors on the reversible dissociation of Escherichia coli ribosomes. Journal of Molecular Biology 94, 461-478.

Gualerzi, C.O., and Pon, C.L. (1990). Initiation of mRNA translation in prokaryotes. Biochemistry 29, 5881-5889. 
Gualerzi, C., Risuleo, G., and Pon, C.L. (1977). Initial rate kinetic analysis of the mechanism of initiation complex formation and the role of initiation factor IF-3. Biochemistry 16, 1684-1689.

Gualerzi, C.O., Spurio, R., La Teana, A., Calogero, R., Celano, B., and Pon, C.L. (1989). Sitedirected mutagenesis of Escherichia coli translation initiation factor IF1. Identification of the amino acid involved in its ribosomal binding and recycling. Protein Engineering 3, 133-138.

Gualerzi, C.O., Severini, M., Spurio, R., La Teana, A., and Pon, C.L. (1991). Molecular dissection of translation initiation factor IF2. Evidence for two structural and functional domains. The Journal of Biological Chemistry 266, 16356-16362.

Gualerzi, C.O., Brandi, L., Caserta, E., Garofalo, C., Lammi, M., La Teana, A., Petrelli, D., Spurio, R., Tomsic, J., and Pon, C.L. (2001). Initiation factors in the early events of mRNA translation in bacteria. Cold Spring Harbor Symposia on Quantitative Biology 66, 363376.

Guenneugues, M., Caserta, E., Brandi, L., Spurio, R., Meunier, S., Pon, C.L., Boelens, R., and Gualerzi, C.O. (2000). Mapping the fMet-tRNA(f)(Met) binding site of initiation factor IF2. The EMBO Journal 19, 5233-5240.

Guillon, J.M., Mechulam, Y., Schmitter, J.M., Blanquet, S., and Fayat, G. (1992). Disruption of the gene for Met-tRNA(fMet) formyltransferase severely impairs growth of Escherichia coli. Journal of Bacteriology 174, 4294-4301.

Guillon, J.M., Heiss, S., Soutourina, J., Mechulam, Y., Laalami, S., Grunberg-Manago, M., and Blanquet, S. (1996). Interplay of methionine tRNAs with translation elongation factor Tu and translation initiation factor 2 in Escherichia coli. The Journal of Biological Chemistry 271, 22321-22325.

Haggerty, T.J., and Lovett, S.T. (1997). IF3-mediated suppression of a GUA initiation codon mutation in the recJ gene of Escherichia coli. Journal of Bacteriology 179, 67056713.

Hansen, P.K., Wikman, F., Clark, B.F., Hershey, J.W., and Uffe Petersen, H. (1986). Interaction between initiator Met-tRNAfMet and elongation factor EF-Tu from E. coli. Biochimie 68, 697-703.

Hartz, D., McPheeters, D.S., and Gold, L. (1989). Selection of the initiator tRNA by Escherichia coli initiation factors. Genes \& Development 3, 1899-1912. 
Hartz, D., Binkley, J., Hollingsworth, T., and Gold, L. (1990). Domains of initiator tRNA and initiation codon crucial for initiator tRNA selection by Escherichia coli IF3. Genes \& Development 4, 1790-1800.

Hauryliuk, V., Mitkevich, V. a, Draycheva, A., Tankov, S., Shyp, V., Ermakov, A., Kulikova, A. a, Makarov, A. a, and Ehrenberg, M. (2009). Thermodynamics of GTP and GDP binding to bacterial initiation factor 2 suggests two types of structural transitions. Journal of Molecular Biology 394, 621-626.

Helgstrand, M., Mandava, C.S., Mulder, F. a a, Liljas, A., Sanyal, S., and Akke, M. (2007). The ribosomal stalk binds to translation factors IF2, EF-Tu, EF-G and RF3 via a conserved region of the L12 C-terminal domain. Journal of Molecular Biology 365, 468-479.

Hennelly, S.P., Antoun, A., Ehrenberg, M., Gualerzi, C.O., Knight, W., Lodmell, J.S., and Hill, W.E. (2005). A time-resolved investigation of ribosomal subunit association. Journal of Molecular Biology 346, 1243-1258.

Hershey, J.W., Yanov, J., Johnston, K., and Fakunding, J.L. (1977). Purification and characterization of protein synthesis initiation factors IF1, IF2, and IF3 from Escherichia coli. Archives of Biochemistry and Biophysics 182, 626-638.

Hirel, P.H., Lévêque, F., Mellot, P., Dardel, F., Panvert, M., Mechulam, Y., and Fayat, G. (1988). Genetic engineering of methionyl-tRNA synthetase: in vitro regeneration of an active synthetase by proteolytic cleavage of a methionyl-tRNA synthetase--betagalactosidase chimeric protein. Biochimie 70, 773-782.

Hirokawa, G., Kiel, M.C., Muto, A., Selmer, M., Raj, V.S., Liljas, A., Igarashi, K., Kaji, H., and Kaji, A. (2002). Post-termination complex disassembly by ribosome recycling factor, a functional tRNA mimic. The EMBO Journal 21, 2272-2281.

Hirose, T., Ideue, T., Wakasugi, T., and Sugiura, M. (1999). The chloroplast infA gene with a functional UUG initiation codon. FEBS Letters 445, 169-172.

Hoe, N.P., and Goguen, J.D. (1993). Temperature sensing in Yersinia pestis: translation of the LcrF activator protein is thermally regulated. Journal of Bacteriology 175, 79017909.

Howe, J.G., and Hershey, J.W. (1983). Initiation factor and ribosome levels are coordinately controlled in Escherichia coli growing at different rates. The Journal of Biological Chemistry 258, 1954-1959. 
Hua, Y., and Raleigh, D.P. (1998). On the global architecture of initiation factor IF3: a comparative study of the linker regions from the Escherichia coli protein and the Bacillus stearothermophilus protein. Journal of Molecular Biology 278, 871-878.

Huang, C., Mandava, C.S., and Sanyal, S. (2010). The ribosomal stalk plays a key role in IF2-mediated association of the ribosomal subunits. Journal of Molecular Biology 399, 145-153.

Iwasaki, K., Sabol, S., Wahba, A.J., and Ochoa, S. (1968). Translation of the genetic message. VII. Role of initiation factors in formation of the chain initiation complex with Escherichia coli ribosomes. Archives of Biochemistry and Biophysics 125, 542-547.

Jenner, L., Romby, P., Rees, B., Schulze-Briese, C., Springer, M., Ehresmann, C., Ehresmann, B., Moras, D., Yusupova, G., and Yusupov, M. (2005). Translational operator of mRNA on the ribosome: how repressor proteins exclude ribosome binding. Science (New York, N.Y.) 308, 120-123.

Jenner, L., Starosta, A.L., Terry, D.S., Mikolajka, A., Filonava, L., Yusupov, M., Blanchard, S.C., Wilson, D.N., and Yusupova, G. (2013). Structural basis for potent inhibitory activity of the antibiotic tigecycline during protein synthesis. Proceedings of the National Academy of Sciences of the United States of America 1196, 1-5.

Jin, H., Zhao, Q., Gonzalez de Valdivia, E.I., Ardell, D.H., Stenström, M., and Isaksson, L. a (2006). Influences on gene expression in vivo by a Shine-Dalgarno sequence. Molecular Microbiology 60, 480-492.

Jin, H., Kelley, A.C., and Ramakrishnan, V. (2011). Crystal structure of the hybrid state of ribosome in complex with the guanosine triphosphatase release factor 3. Proceedings of the National Academy of Sciences of the United States of America 108, 15798-15803.

Johansson, J., Mandin, P., Renzoni, A., Chiaruttini, C., Springer, M., and Cossart, P. (2002). An RNA thermosensor controls expression of virulence genes in Listeria monocytogenes. Cell 110, 551-561.

Julián, P., Milon, P., Agirrezabala, X., Lasso, G., Gil, D., Rodnina, M. V, and Valle, M. (2011). The Cryo-EM structure of a complete $30 \mathrm{~S}$ translation initiation complex from Escherichia coli. PLoS Biology 9, e1001095.

Kaminishi, T., Wilson, D.N., Takemoto, C., Harms, J.M., Kawazoe, M., Schluenzen, F., Hanawa-Suetsugu, K., Shirouzu, M., Fucini, P., and Yokoyama, S. (2007). A snapshot of the $30 \mathrm{~S}$ ribosomal subunit capturing mRNA via the Shine-Dalgarno interaction. Structure (London, England : 1993) 15, 289-297. 
Kapralou, S., Fabbretti, A., Garulli, C., Spurio, R., Gualerzi, C.O., Dahlberg, A.E., and Pon, C.L. (2008). Translation initiation factor IF1 of Bacillus stearothermophilus and Thermus thermophilus substitute for Escherichia coli IF1 in vivo and in vitro without a direct IF1IF2 interaction. Molecular Microbiology 70, 1368-1377.

Kapralou, S., Fabbretti, A., Garulli, C., Gualerzi, C.O., Pon, C.L., and Spurio, R. (2009). Characterization of Bacillus stearothermophilus infA and of its product IF1. Gene 428, 31-35.

Karimi, R., Pavlov, M.Y., Buckingham, R.H., and Ehrenberg, M. (1999). Novel roles for classical factors at the interface between translation termination and initiation. Molecular Cell 3, 601-609.

Kay, A.C., and Grunberg-Manago, M. (1972). The mechanism of action of initiation factor IF1: non-analogy with elongation factor EFTs. Biochimica Et Biophysica Acta 277, 225230.

Kitakawa, M., and Isono, K. (1982). An amber mutation in the gene rpsA for ribosomal protein S1 in Escherichia coli. Molecular \& General Genetics : MGG 185, 445-447.

Komarova, A. V, Tchufistova, L.S., Supina, E. V, and Boni, I. V (2002). Protein S1 counteracts the inhibitory effect of the extended Shine-Dalgarno sequence on translation. RNA (New York, N.Y.) 8, 1137-1147.

Kozak, M. (1983). Comparison of initiation of protein synthesis in procaryotes, eucaryotes, and organelles. Microbiological Reviews 47, 1-45.

Kozak, M. (1999). Initiation of translation in prokaryotes and eukaryotes. Gene 234, 187-208.

Kudla, G., Murray, A., Tollervey, D., and Plotkin, J. (2009). Coding-sequence determinants of gene expression in Escherichia coli. Science 255-258.

Kyrpides, N.C., and Woese, C.R. (1998). Universally conserved translation initiation factors. Proceedings of the National Academy of Sciences of the United States of America 95, 224-228.

Laalami, S., Putzer, H., Plumbridge, J. a, and Grunberg-Manago, M. (1991). A severely truncated form of translational initiation factor 2 supports growth of Escherichia coli. Journal of Molecular Biology 220, 335-349. 
Laalami, S., Timofeev, a V, Putzer, H., Leautey, J., and Grunberg-Manago, M. (1994). In vivo study of engineered G-domain mutants of Escherichia coli translation initiation factor IF2. Molecular Microbiology 11, 293-302.

Lang, V., Gualerzi, C., and McCarthy, J.E. (1989). Ribosomal affinity and translational initiation in Escherichia coli. In vitro investigations using translational initiation regions of differing efficiencies from the atp operon. Journal of Molecular Biology 210, 659-663.

Laursen, B.S., Mortensen, K.K., Sperling-Petersen, H.U., and Hoffman, D.W. (2003). A conserved structural motif at the $\mathrm{N}$ terminus of bacterial translation initiation factor IF2. The Journal of Biological Chemistry 278, 16320-16328.

Laursen, B.S., Kjaergaard, A.C., Mortensen, K.K., Hoffman, D.W., and Sperling-Petersen, H.U. (2004). The N-terminal domain (IF2N) of bacterial translation initiation factor IF2 is connected to the conserved C-terminal domains by a flexible linker. Protein Science : a Publication of the Protein Society 13, 230-239.

Laursen, B.S., Sørensen, H.P., Mortensen, K.K., and Sperling-Petersen, H.U. (2005). Initiation of protein synthesis in bacteria. Microbiology and Molecular Biology Reviews : MMBR 69, 101-123.

Lee, K., Holland-Staley, C. a, and Cunningham, P.R. (1996). Genetic analysis of the ShineDalgarno interaction: selection of alternative functional mRNA-rRNA combinations. RNA (New York, N.Y.) 2, 1270-1285.

Lin, Q., Yu, N.J., and Spremulli, L.L. (1996). Expression and functional analysis of Euglena Gracilis chloroplast initiation factor 3. Plant Molecular Biology 32, 937-945.

Lockwood, a H., Chakraborty, P.R., and Maitra, U. (1971). A complex between initiation factor IF2, guanosine triphosphate, and fMet-tRNA: an intermediate in initiation complex formation. Proceedings of the National Academy of Sciences of the United States of America 68, 3122-3126.

Lomakin, I.B., Shirokikh, N.E., Yusupov, M.M., Hellen, C.U.T., and Pestova, T. V (2006). The fidelity of translation initiation: reciprocal activities of eIF1, IF3 and YciH. The EMBO Journal 25, 196-210.

Luchin, S., Putzer, H., Hershey, J.W., Cenatiempo, Y., Grunberg-Manago, M., and Laalami, S. (1999). In vitro study of two dominant inhibitory GTPase mutants of Escherichia coli translation initiation factor IF2. Direct evidence that GTP hydrolysis is necessary for factor recycling. The Journal of Biological Chemistry 274, 6074-6079. 
Ma, J., Campbell, A., and Karlin, S. (2002). Correlations between Shine-Dalgarno sequences and gene features such as predicted expression levels and operon structures. Journal of Bacteriology 184, 5733-5745.

Maar, D., Liveris, D., Sussman, J.K., Ringquist, S., Moll, I., Heredia, N., Kil, A., Bläsi, U., Schwartz, I., and Simons, R.W. (2008). A single mutation in the IF3 N-terminal domain perturbs the fidelity of translation initiation at three levels. Journal of Molecular Biology 383, 937-944.

Majumdar, A., Bose, K.K., and Gupta, N.K. (1976). Specific binding of Excherichia coli chain Initiation factor 2 to fMet-tRnafMet. The Journal of Biological Chemistry 251, 137140.

Mandal, M., and Breaker, R.R. (2004). Adenine riboswitches and gene activation by disruption of a transcription terminator. Nature Structural \& Molecular Biology 11, 2935.

Mandal, M., Boese, B., Barrick, J.E., Winkler, W.C., and Breaker, R.R. (2003). Riboswitches control fundamental biochemical pathways in Bacillus subtilis and other bacteria. Cell 113, 577-586.

Mandal, N., Mangroo, D., Dalluge, J.J., McCloskey, J. a, and Rajbhandary, U.L. (1996). Role of the three consecutive $\mathrm{G}: \mathrm{C}$ base pairs conserved in the anticodon stem of initiator tRNAs in initiation of protein synthesis in Escherichia coli. RNA (New York, N.Y.) 2, 473482.

Mandava, C.S., Peisker, K., Ederth, J., Kumar, R., Ge, X., Szaflarski, W., and Sanyal, S. (2012). Bacterial ribosome requires multiple L12 dimers for efficient initiation and elongation of protein synthesis involving IF2 and EF-G. Nucleic Acids Research 40, 20542064.

Marshall, R.A., Aitken, C.E., and Puglisi, J.D. (2009). GTP hydrolysis by IF2 guides progression of the ribosome into elongation. Molecular Cell 35, 37-47.

Marzi, S., Knight, W., Brandi, L., Caserta, E., Soboleva, N., Hill, W.E., Gualerzi, C.O., and Lodmell, J.S. (2003). Ribosomal localization of translation initiation factor IF2. RNA (New York, N.Y.) 9, 958-969.

Marzi, S., Myasnikov, A.G., Serganov, A., Ehresmann, C., Romby, P., Yusupov, M., and Klaholz, B.P. (2007). Structured mRNAs regulate translation initiation by binding to the platform of the ribosome. Cell 130, 1019-1031. 
Mayer, C., Köhrer, C., Kenny, E., Prusko, C., and RajBhandary, U.L. (2003). Anticodon sequence mutants of Escherichia coli initiator tRNA: effects of overproduction of aminoacyl-tRNA synthetases, methionyl-tRNA formyltransferase, and initiation factor 2 on activity in initiation. Biochemistry 42, 4787-4799.

McCarthy, J.E., and Brimacombe, R. (1994). Prokaryotic translation: the interactive pathway leading to initiation. Trends in Genetics : TIG 10, 402-407.

McCarthy, J.E., and Gualerzi, C. (1990). Translational control of prokaryotic gene expression. Trends in Genetics : TIG 6, 78-85.

McCutcheon, J.P., Agrawal, R.K., Philips, S.M., Grassucci, R. a, Gerchman, S.E., Clemons, W.M., Ramakrishnan, V., and Frank, J. (1999). Location of translational initiation factor IF3 on the small ribosomal subunit. Proceedings of the National Academy of Sciences of the United States of America 96, 4301-4306.

Mechulam, Y., Schmitt, E., Maveyraud, L., Zelwer, C., Nureki, O., Yokoyama, S., Konno, M., and Blanquet, S. (1999). Crystal structure of Escherichia coli methionyl-tRNA synthetase highlights species-specific features. Journal of Molecular Biology 294, 12871297.

Mechulam, Y., Guillon, L., Yatime, L., Blanquet, S., and Schmitt, E. (2007). Protectionbased assays to measure aminoacyl-tRNA binding to translation initiation factors. Methods in Enzymology 430, 265-281.

Meinnel, T., Guillon, J.M., Mechulam, Y., and Blanquet, S. (1993). The Escherichia coli fmt gene, encoding methionyl-tRNA(fMet) formyltransferase, escapes metabolic control. Journal of Bacteriology 175, 993-1000.

Meinnel, T., Sacerdot, C., Graffe, M., Blanquet, S., and Springer, M. (1999). Discrimination by Escherichia coli initiation factor IF3 against initiation on non-canonical codons relies on complementarity rules. Journal of Molecular Biology 290, 825-837.

Melançon, P., Leclerc, D., Destroismaisons, N., and Brakier-Gingras, L. (1990). The antiShine-Dalgarno region in Escherichia coli 16S ribosomal RNA is not essential for the correct selection of translational starts. Biochemistry 29, 3402-3407.

Mellot, P., Mechulam, Y., Le Corre, D., Blanquet, S., and Fayat, G. (1989). Identification of an amino acid region supporting specific methionyl-tRNA synthetase: tRNA recognition. Journal of Molecular Biology 208, 429-443. 
Meunier, S., Spurio, R., Czisch, M., Wechselberger, R., Guenneugues, M., Gualerzi, C.O., and Boelens, R. (2000). Structure of the fMet-tRNA(fMet)-binding domain of B. stearothermophilus initiation factor IF2. The EMBO Journal 19, 1918-1926.

Miller, M., and Wahba, A. (1973). Chain initiation factor 2. Journal of Biological Chemistry.

Milon, P., Tischenko, E., Tomsic, J., Caserta, E., Folkers, G., La Teana, A., Rodnina, M. V, Pon, C.L., Boelens, R., and Gualerzi, C.O. (2006). The nucleotide-binding site of bacterial translation initiation factor 2 (IF2) as a metabolic sensor. Proceedings of the National Academy of Sciences of the United States of America 103, 13962-13967.

Milon, P., Konevega, A.L., Peske, F., Fabbretti, A., Gualerzi, C.O., and Rodnina, M. V (2007). Transient kinetics, fluorescence, and FRET in studies of initiation of translation in bacteria. Methods in Enzymology 430, 1-30.

Milon, P., Konevega, A.L., Gualerzi, C.O., and Rodnina, M. V (2008). Kinetic checkpoint at a late step in translation initiation. Molecular Cell 30, 712-720.

Milon, P., Carotti, M., Konevega, A.L., Wintermeyer, W., Rodnina, M. V, and Gualerzi, C.O. (2010). The ribosome-bound initiation factor 2 recruits initiator tRNA to the 30S initiation complex. EMBO Reports 11, 312-316.

Milón, P., and Rodnina, M. V (2012). Kinetic control of translation initiation in bacteria. Critical Reviews in Biochemistry and Molecular Biology 47, 334-348.

Milón, P., Maracci, C., Filonava, L., Gualerzi, C.O., and Rodnina, M. V (2012a). Real-time assembly landscape of bacterial $30 \mathrm{~S}$ translation initiation complex. Nature Structural \& Molecular Biology 19, 609-615.

Milón, P., Maracci, C., Filonava, L., Gualerzi, C.O., and Rodnina, M. V (2012b). Real-time assembly landscape of bacterial $30 \mathrm{~S}$ translation initiation complex. Nature Structural \& Molecular Biology 19, 609-615.

Misselwitz, R., Welfe, K., Krafft, C., Gualerzi, C.O., and Welfle, H. (1997). Translational initiation factor IF2 from Bacillus stearothermophilus: a spectroscopic and microcalorimetric study of the C-domain. Biochemistry 36, 3170-3178.

Moazed, D., Samaha, R.R., Gualerzi, C., and Noller, H.F. (1995). Specific protection of 16 $S$ rRNA by translational initiation factors. Journal of Molecular Biology 248, 207-210. 
Moreau, M., De Cock, E., Fortier, P.L., Garcia, C., Albaret, C., Blanquet, S., Lallemand, J.Y., and Dardel, F. (1997). Heteronuclear NMR studies of E. coli translation initiation factor IF3. Evidence that the inter-domain region is disordered in solution. Journal of Molecular Biology 266, 15-22.

Morel-Deville, F., Vachon, G., Sacerdot, C., Cozzone, a J., Grunberg-Manago, M., and Cenatiempo, Y. (1990). Characterization of the translational start site for IF2 beta, a short form of Escherichia coli initiation factor IF2. European Journal of Biochemistry / FEBS 188, 605-614.

Moreno, J.M., Drskjøtersen, L., Kristensen, J.E., Mortensen, K.K., and Sperling-Petersen, H.U. (1999). Characterization of the domains of E. coli initiation factor IF2 responsible for recognition of the ribosome. FEBS Letters 455, 130-134.

Mortensen, K.K., Hajnsdorf, E., Regnier, P., and Sperling-Petersen, H.U. (1995). Improved recombinant tandem expression of translation initiation factor IF2 in RNASE E deficient E. coli cells. Biochemical and Biophysical Research Communications 214, 1254-1259.

Mortensen, K.K., Kildsgaard, J., Moreno, J.M., Steffensen, S. a, Egebjerg, J., and SperlingPetersen, H.U. (1998). A six-domain structural model for Escherichia coli translation initiation factor IF2. Characterisation of twelve surface epitopes. Biochemistry and Molecular Biology International 46, 1027-1041.

Mukundan, M.A., Hershey, J.W., Dewey, K.F., and Thach, R.E. (1968). Binding of formylmethionyl-tRNA to 30S ribosomal sub-units. Nature 217, 1013-1016.

Murzin, A.G. (1993). OB(oligonucleotide/oligosaccharide binding)-fold: common structural and functional solution for non-homologous sequences. The EMBO Journal 12, 861-867.

Myasnikov, A.G., Marzi, S., Simonetti, A., Giuliodori, A.M., Gualerzi, C.O., Yusupova, G., Yusupov, M., and Klaholz, B.P. (2005). Conformational transition of initiation factor 2 from the GTP- to GDP-bound state visualized on the ribosome. Nature Structural \& Molecular Biology 12, 1145-1149.

Nakamoto, T. (2006). A unified view of the initiation of protein synthesis. Biochemical and Biophysical Research Communications 341, 675-678.

Nakamoto, T. (2009). Evolution and the universality of the mechanism of initiation of protein synthesis. Gene 432, 1-6. 
Nissen, P. (2000). The Structural Basis of Ribosome Activity in Peptide Bond Synthesis. Science 289, 920-930.

Nissen, P., Kjeldgaard, M., Thirup, S., Polekhina, G., Reshetnikova, L., Clark, B.F., and Nyborg, J. (1995). Crystal structure of the ternary complex of Phe-tRNAPhe, EF-Tu, and a GTP analog. Science (New York, N.Y.) 270, 1464-1472.

Nomura, M., Gourse, R., and Baughman, G. (1984). Regulation of the synthesis of ribosomes and ribosomal components. Annual Review of Biochemistry 53, 75-117.

Nyengaard, N., and Mortensen, K. (1991). Tandem translation of E. coli initiation factor IF2beta: Purification and characterization in vitro of two active forms. Biochemical and ... $181,1572-1579$.

Olsson, C.L., Graffe, M., Springer, M., and Hershey, J.W. (1996). Physiological effects of translation initiation factor IF3 and ribosomal protein L20 limitation in Escherichia coli. Molecular \& General Genetics : MGG 250, 705-714.

Passalacqua, K.D., Varadarajan, A., Ondov, B.D., Okou, D.T., Zwick, M.E., and Bergman, N.H. (2009). Structure and complexity of a bacterial transcriptome. Journal of Bacteriology 191, 3203-3211.

Peske, F., Savelsbergh, A., Katunin, V.I., Rodnina, M. V, and Wintermeyer, W. (2004). Conformational changes of the small ribosomal subunit during elongation factor $\mathrm{G}$ dependent tRNA-mRNA translocation. Journal of Molecular Biology 343, 1183-1194.

Peske, F., Rodnina, M. V, and Wintermeyer, W. (2005). Sequence of steps in ribosome recycling as defined by kinetic analysis. Molecular Cell 18, 403-412.

Pestka, S. (1971). Inhibitors of ribosome functions. Annual Review of Microbiology 25, 487-562.

Pestova, T. V, Lomakin, I.B., Lee, J.H., Choi, S.K., Dever, T.E., and Hellen, C.U. (2000). The joining of ribosomal subunits in eukaryotes requires elF5B. Nature 403, 332-335.

Petersen, H.U., Røll, T., Grunberg-Manago, M., and Clark, B.F. (1979). Specific interaction of initiation factor IF2 of $E$. coli with formylmethionyl-tRNA $f$ Met. Biochemical and Biophysical Research Communications 91, 1068-1074.

Petersen, H.U., Kruse, T.A., Worm-Leonhard, H., Siboska, G.E., Clark, B.F., Boutorin, A.S., Remy, P., Ebel, J.P., Dondon, J., and Grunberg-Manago, M. (1981). Study of the 
interaction of Escherichia coli initiation factor IF2 with formylmethionyl-tRNAMetf by partial digestion with cobra venom ribonuclease. FEBS Letters 128, 161-165.

Petrelli, D., LaTeana, a, Garofalo, C., Spurio, R., Pon, C.L., and Gualerzi, C.O. (2001). Translation initiation factor IF3: two domains, five functions, one mechanism? The EMBO Journal 20, 4560-4569.

Petrelli, D., Garofalo, C., Lammi, M., Spurio, R., Pon, C.L., Gualerzi, C.O., and Teana, A. La (2003). Mapping the Active Sites of Bacterial Translation Initiation Factor IF3. Journal of Molecular Biology 331, 541-556.

Phadtare, S., Kazakov, T., Bubunenko, M., Court, D.L., Pestova, T., and Severinov, K. (2007). Transcription antitermination by translation initiation factor IF1. Journal of Bacteriology 189, 4087-4093.

Pioletti, M., Schlünzen, F., Harms, J., Zarivach, R., Glühmann, M., Avila, H., Bashan, a, Bartels, H., Auerbach, T., Jacobi, C., et al. (2001). Crystal structures of complexes of the small ribosomal subunit with tetracycline, edeine and IF3. The EMBO Journal 20, 18291839.

Pisareva, V.P., Pisarev, A. V, Hellen, C.U.T., Rodnina, M. V, and Pestova, T. V (2006). Kinetic analysis of interaction of eukaryotic release factor 3 with guanine nucleotides. The Journal of Biological Chemistry 281, 40224-40235.

Pisareva, V.P., Hellen, C.U.T., and Pestova, T. V (2007). Kinetic analysis of the interaction of guanine nucleotides with eukaryotic translation initiation factor elF5B. Biochemistry $46,2622-2629$.

Plumbridge, J., and Deville, F. (1985). Two translational initiation sites in the infB gene are used to express initiation factor IF2 alpha and IF2 beta in Escherichia coli. The EMBO ... 4, 223-229.

Plumbridge, J. a, Howe, J.G., Springer, M., Touati-Schwartz, D., Hershey, J.W., and Grunberg-Manago, M. (1982). Cloning and mapping of a gene for translational initiation factor IF2 in Escherichia coli. Proceedings of the National Academy of Sciences of the United States of America 79, 5033-5037.

Polard, P., Prère, M.F., Chandler, M., and Fayet, O. (1991). Programmed translational frameshifting and initiation at an AUU codon in gene expression of bacterial insertion sequence IS911. Journal of Molecular Biology 222, 465-477. 
Pon, C.L., and Gualerzi, C.O. (1984). Mechanism of protein biosynthesis in prokaryotic cells. FEBS Letters 175, 203-207.

Pon, C., Paci, M., Pawlik, R., and Gualerzi, C. (1985). Structure-function relationship in Escherichia coli initiation factors. Biochemical and biophysical characterization of the interaction between IF-2 and guanosine nucleotides. Journal of Biological Chemistry 8918-8924.

Qin, D., and Fredrick, K. (2009). Control of translation initiation involves a factor-induced rearrangement of helix 44 of $16 \mathrm{~S}$ ribosomal RNA. Molecular Microbiology 71, 12391249.

Qin, D., Abdi, N., and Fredrick, K. (2007). Characterization of 16S rRNA mutations that decrease the fidelity of translation initiation. Rna 2348-2355.

RajBhandary, U. (1994). Initiator transfer RNAs. Journal of Bacteriology 176, 547-552.

Rasmussen, L., and Oliveira, C. (2008). Solution Structure of C-Terminal Escherichia coli Translation Initiation Factor IF2 by Small-Angle X-ray Scattering†. Biochemistry 55905598.

Revel, M., Lelong, J.C., Brawerman, G., and Gros, F. (1968). Function of three protein factors and ribosomal subunits in the initiation of protein synthesis in E. coli. Nature 219, 1016-1021.

Rich, A., and RajBhandary, U.L. (1976). Transfer RNA: molecular structure, sequence, and properties. Annual Review of Biochemistry 45, 805-860.

Ringquist, S., Jones, T., Snyder, E.E., Gibson, T., Boni, I., and Gold, L. (1995). High-affinity RNA ligands to Escherichia coli ribosomes and ribosomal protein S1: comparison of natural and unnatural binding sites. Biochemistry 34, 3640-3648.

Rinke-Appel, J., Jünke, N., Brimacombe, R., Lavrik, I., Dokudovskaya, S., Dontsova, O., and Bogdanov, A. (1994). Contacts between $16 \mathrm{~S}$ ribosomal RNA and mRNA, within the spacer region separating the AUG initiator codon and the Shine-Dalgarno sequence; a site-directed cross-linking study. Nucleic Acids Research 22, 3018-3025.

Risuleo, G., Gualerzi, C., and Pon, C. (1976). Specificity and properties of the destabilization, induced by initiation factor IF-3, of ternary complexes of the 30-S ribosomal subunit, aminoacyl-tRNA and polynucleotides. European Journal of Biochemistry / FEBS 67, 603-613. 
Rodnina, M. V, Savelsbergh, A., Katunin, V.I., and Wintermeyer, W. (1997). Hydrolysis of GTP by elongation factor $\mathrm{G}$ drives tRNA movement on the ribosome. Nature 385, 37-41.

Rodnina, M. V, Stark, H., Wieden, H., Matassova, N.B., Peske, F., Daviter, T., and Gualerzi, C.O. (2000). GTPase Mechanisms and Functions of Translation Factors on the Ribosome. 381, 377-387.

Roll-Mecak, a, Cao, C., Dever, T.E., and Burley, S.K. (2000). X-Ray structures of the universal translation initiation factor IF2/elF5B: conformational changes on GDP and GTP binding. Cell 103, 781-792.

Sabol, S., Sillero, M.A., Iwasaki, K., and Ochoa, S. (1970). Purification and properties of initiation factor F3. Nature 228, 1269-1273.

Sacerdot, C., Fayat, G., Dessen, P., Springer, M., Plumbridge, J.A., Grunberg-Manago, M., and Blanquet, S. (1982). Sequence of a 1.26-kb DNA fragment containing the structural gene for E.coli initiation factor IF3: presence of an AUU initiator codon. The EMBO Journal 1, 311-315.

Sacerdot, C., Vachon, G., Laalami, S., Morel-Deville, F., Cenatiempo, Y., and GrunbergManago, M. (1992). Both forms of translational initiation factor IF2 (alpha and beta) are required for maximal growth of Escherichia coli. Evidence for two translational initiation codons for IF2 beta. Journal of Molecular Biology 225, 67-80.

Sacerdot, C., Chiaruttini, C., Engst, K., Graffe, M., Milet, M., Mathy, N., Dondon, J., and Springer, M. (1996). The role of the AUU initiation codon in the negative feedback regulation of the gene for translation initiation factor IF3 in Escherichia coli. Molecular Microbiology 21, 331-346.

Saraiya, A.A., Lamichhane, T.N., Chow, C.S., SantaLucia, J., and Cunningham, P.R. (2008). Identification and role of functionally important motifs in the 970 loop of Escherichia coli 16S ribosomal RNA. Journal of Molecular Biology 376, 645-657.

Savelsbergh, a, Mohr, D., Wilden, B., Wintermeyer, W., and Rodnina, M. V (2000). Stimulation of the GTPase activity of translation elongation factor $G$ by ribosomal protein L7/12. The Journal of Biological Chemistry 275, 890-894.

Savelsbergh, A., Katunin, V.I., Mohr, D., Peske, F., Rodnina, M. V, and Wintermeyer, W. (2003). An elongation factor $G$-induced ribosome rearrangement precedes tRNA-mRNA translocation. Molecular Cell 11, 1517-1523. 
Scherer, G.F., Walkinshaw, M.D., Arnott, S., and Morré, D.J. (1980). The ribosome binding sites recognized by E. coli ribosomes have regions with signal character in both the leader and protein coding segments. Nucleic Acids Research 8, 3895-3907.

Schleich, T., Verwolf, G.L., and Twombly, K. (1980). A circular dichroism study of Escherichia coli Initiation Factor-1 binding to polynucleotides. Biochimica Et Biophysica Acta 609, 313-320.

Schmeing, T.M., and Ramakrishnan, V. (2009). What recent ribosome structures have revealed about the mechanism of translation. Nature 461, 1234-1242.

Schmeing, T.M., Voorhees, R.M., Kelley, A.C., Gao, Y.-G., Murphy, F. V, Weir, J.R., and Ramakrishnan, V. (2009). The crystal structure of the ribosome bound to EF-Tu and aminoacyl-tRNA. Science (New York, N.Y.) 326, 688-694.

Schmitt, E., Mechulam, Y., Ruff, M., Mitschler, A., Moras, D., and Blanquet, S. (1996a). Crystallization and preliminary X-ray analysis of Escherichia coli methionyl-tRNA(fMet) formyltransferase. Proteins 25, 139-141.

Schmitt, E., Blanquet, S., and Mechulam, Y. (1996b). Structure of crystalline Escherichia coli methionyl-tRNA(f)Met formyltransferase: comparison with glycinamide ribonucleotide formyltransferase. The EMBO Journal 15, 4749-4758.

Schmitt, E., Panvert, M., Blanquet, S., and Mechulam, Y. (1998). Crystal structure of methionyl-tRNAfMet transformylase complexed with the initiator formyl-methionyltRNAfMet. The EMBO Journal 17, 6819-6826.

Schmitt, E., Blanquet, S., and Mechulam, Y. (1999). Crystallization and preliminary X-ray analysis of Escherichia coli methionyl-tRNAMet(f) formyltransferase complexed with formyl-methionyl-tRNAMet(f). Acta Crystallographica. Section D, Biological Crystallography 55, 332-334.

Schneider, T.D., Stormo, G.D., Gold, L., and Ehrenfeucht, A. (1986). Information content of binding sites on nucleotide sequences. Journal of Molecular Biology 188, 415-431.

Schuwirth, B.S., Borovinskaya, M. a, Hau, C.W., Zhang, W., Vila-Sanjurjo, A., Holton, J.M., and Cate, J.H.D. (2005). Structures of the bacterial ribosome at $3.5 \mathrm{~A}$ resolution. Science (New York, N.Y.) 310, 827-834.

Schweisguth, D.C., and Moore, P.B. (1997). On the conformation of the anticodon loops of initiator and elongator methionine tRNAs. Journal of Molecular Biology 267, 505-519. 
Selmer, M., Dunham, C.M., Murphy, F. V, Weixlbaumer, A., Petry, S., Kelley, A.C., Weir, J.R., and Ramakrishnan, V. (2006). Structure of the $70 \mathrm{~S}$ ribosome complexed with mRNA and tRNA. Science (New York, N.Y.) 313, 1935-1942.

Selmer, M., Gao, Y.-G., Weixlbaumer, A., and Ramakrishnan, V. (2012). Ribosome engineering to promote new crystal forms. Acta Crystallographica. Section D, Biological Crystallography 68, 578-583.

Sengupta, J., Agrawal, R.K., and Frank, J. (2001). Visualization of protein S1 within the $30 \mathrm{~S}$ ribosomal subunit and its interaction with messenger RNA. Proceedings of the National Academy of Sciences of the United States of America 98, 11991-11996.

Seong, B.L., and RajBhandary, U.L. (1987). Escherichia coli formylmethionine tRNA: mutations in GGGCCC sequence conserved in anticodon stem of initiator tRNAs affect initiation of protein synthesis and conformation of anticodon loop. Proceedings of the National Academy of Sciences of the United States of America 84, 334-338.

Sette, M., Van Tilborg, P., Spurio, R., Kaptein, R., Paci, M., Gualerzi, C.O., and Boelens, R. (1997). The structure of the translational initiation factor IF1 from E.coli contains an oligomer-binding motif. The EMBO Journal 16, 1436-1443.

Sette, M., Spurio, R., Van Tilborg, P., Gualerzi, C.O., and Boelens, R. (1999). Identification of the ribosome binding sites of translation initiation factor IF3 by multidimensional heteronuclear NMR spectroscopy. RNA (New York, N.Y.) 5, 82-92.

Severini, M., Spurio, R., La Teana, a, Pon, C.L., and Gualerzi, C.O. (1991). Ribosomeindependent GTPase activity of translation initiation factor IF2 and of its G-domain. The Journal of Biological Chemistry 266, 22800-22802.

Shimizu, Y., Inoue, a, Tomari, Y., Suzuki, T., Yokogawa, T., Nishikawa, K., and Ueda, T. (2001). Cell-free translation reconstituted with purified components. Nature Biotechnology 19, 751-755.

Shine, J., and Dalgarno, L. (1974). The 3'-terminal sequence of Escherichia coli 16S ribosomal RNA: complementarity to nonsense triplets and ribosome binding sites. Proceedings of the National Academy of Sciences of the United States of America 71, 1342-1346.

Simonetti, A., Marzi, S., Myasnikov, A.G., Fabbretti, A., Yusupov, M., Gualerzi, C.O., and Klaholz, B.P. (2008). Structure of the 30 S translation initiation complex. Nature 455, 416-420. 
Simonetti, A., Marzi, S., Jenner, L., Myasnikov, A., Romby, P., Yusupova, G., Klaholz, B.P., and Yusupov, M. (2009). A structural view of translation initiation in bacteria. Cellular and Molecular Life Sciences : CMLS 66, 423-436.

Singh, N.S., Das, G., Seshadri, a, Sangeetha, R., and Varshney, U. (2005). Evidence for a role of initiation factor 3 in recycling of ribosomal complexes stalled on mRNAs in Escherichia coli. Nucleic Acids Research 33, 5591-5601.

Singh, N.S., Ahmad, R., Sangeetha, R., and Varshney, U. (2008). Recycling of ribosomal complexes stalled at the step of elongation in Escherichia coli. Journal of Molecular Biology 380, 451-464.

Skorski, P., Leroy, P., Fayet, O., Dreyfus, M., and Hermann-Le Denmat, S. (2006). The highly efficient translation initiation region from the Escherichia coli rpsA gene lacks a shine-dalgarno element. Journal of Bacteriology 188, 6277-6285.

De Smit, M.H., and Van Duin, J. (1994). Translational initiation on structured messengers. Another role for the Shine-Dalgarno interaction. Journal of Molecular Biology 235, 173-184.

De Smit, M.H., and Van Duin, J. (2003). Translational standby sites: how ribosomes may deal with the rapid folding kinetics of mRNA. Journal of Molecular Biology 331, 737-743.

Sørensen, H.P., Hedegaard, J., Sperling-Petersen, H.U., and Mortensen, K.K. (2001). Remarkable conservation of translation initiation factors: IF1/eIF1A and IF2/elF5B are universally distributed phylogenetic markers. IUBMB Life 51, 321-327.

Sørensen, M. a, Fricke, J., and Pedersen, S. (1998). Ribosomal protein S1 is required for translation of most, if not all, natural mRNAs in Escherichia coli in vivo. Journal of Molecular Biology 280, 561-569.

Spurio, R., Brandi, L., Caserta, E., Pon, C.L., Gualerzi, C.O., Misselwitz, R., Krafft, C., Welfle, K., and Welfle, H. (2000). The C-terminal subdomain (IF2 C-2) contains the entire fMet-tRNA binding site of initiation factor IF2. The Journal of Biological Chemistry 275, 2447-2454.

Steffensen, S.A., Poulsen, A.B., Mortensen, K.K., and Sperling-Petersen, H.U. (1997). E. coli translation initiation factor IF2--an extremely conserved protein. Comparative sequence analysis of the infB gene in clinical isolates of E. coli. FEBS Letters 419, 281284. 
Steitz, J.A. (1969). Polypeptide chain initiation: nucleotide sequences of the three ribosomal binding sites in bacteriophage R17 RNA. Nature 224, 957-964.

Steitz, T.A. (2008). A structural understanding of the dynamic ribosome machine. 9.

Stringer, E.A., Sarkar, P., and Maitra, U. (1977). Function of initiation factor 1 in the binding and release of initiation factor 2 from ribosomal initiation complexes in Escherichia coli. The Journal of Biological Chemistry 252, 1739-1744.

Studer, S.M., and Joseph, S. (2006). Unfolding of mRNA secondary structure by the bacterial translation initiation complex. Molecular Cell 22, 105-115.

Subramanian, A.R., and Davis, B.D. (1970). Activity of initiation factor F3 in dissociating Escherichia coli ribosomes. Nature 228, 1273-1275.

Subramanian, A.R., Ron, E.Z., and Davis, B.D. (1968). A factor required for ribosome dissociation in Escherichia coli. Proceedings of the National Academy of Sciences of the United States of America 61, 761-767.

Sundari, R.M., Stringer, E.A., Schulman, L.H., and Maitra, U. (1976). Interaction of bacterial initiation factor 2 with initiator tRNA. The Journal of Biological Chemistry 251, 3338-3345.

Sussman, J.K., Simons, E.L., and Simons, R.W. (1996). Escherichia coli translation initiation factor 3 discriminates the initiation codon in vivo. Molecular Microbiology 21, 347-360.

Szkaradkiewicz, K., Zuleeg, T., Limmer, S., and Sprinzl, M. (2000). Interaction of fMettRNAfMet and fMet-AMP with the C-terminal domain of Thermus thermophilus translation initiation factor 2. European Journal of Biochemistry / FEBS 267, 4290-4299.

Tapprich, W.E., Goss, D.J., and Dahlberg, a E. (1989). Mutation at position 791 in Escherichia coli 16S ribosomal RNA affects processes involved in the initiation of protein synthesis. Proceedings of the National Academy of Sciences of the United States of America 86, 4927-4931.

La Teana, a, Pon, C.L., and Gualerzi, C.O. (1993). Translation of mRNAs with degenerate initiation triplet AUU displays high initiation factor 2 dependence and is subject to initiation factor 3 repression. Proceedings of the National Academy of Sciences of the United States of America 90, 4161-4165. 
La Teana, a, Pon, C.L., and Gualerzi, C.O. (1996). Late events in translation initiation. Adjustment of fMet-tRNA in the ribosomal P-site. Journal of Molecular Biology 256, 667675.

La Teana, A., Gualerzi, C.O., and Brimacombe, R. (1995). From stand-by to decoding site. Adjustment of the mRNA on the $30 \mathrm{~S}$ ribosomal subunit under the influence of the initiation factors. RNA (New York, N.Y.) 1, 772-782.

La Teana, A., Gualerzi, C.O., and Dahlberg, A.E. (2001). Initiation factor IF 2 binds to the alpha-sarcin loop and helix 89 of Escherichia coli 23S ribosomal RNA. RNA (New York, N.Y.) 7, 1173-1179.

Tolstrup, N., Sensen, C.W., Garrett, R.A., and Clausen, I.G. (2000). Two different and highly organized mechanisms of translation initiation in the archaeon Sulfolobus solfataricus. Extremophiles : Life Under Extreme Conditions 4, 175-179.

Tomsic, J., Vitali, L. a, Daviter, T., Savelsbergh, a, Spurio, R., Striebeck, P., Wintermeyer, W., Rodnina, M. V, and Gualerzi, C.O. (2000). Late events of translation initiation in bacteria: a kinetic analysis. The EMBO Journal 19, 2127-2136.

Tsai, A., Petrov, A., Marshall, R.A., Korlach, J., Uemura, S., and Puglisi, J.D. (2012). Heterogeneous pathways and timing of factor departure during translation initiation. Nature 487, 390-393.

Varshney, U., and RajBhandary, U.L. (1992). Role of methionine and formylation of initiator tRNA in initiation of protein synthesis in Escherichia coli. Journal of Bacteriology 174, 7819-7826.

Varshney, U., Lee, C.P., and RajBhandary, U.L. (1993). From elongator tRNA to initiator tRNA. Proceedings of the National Academy of Sciences of the United States of America 90, 2305-2309.

Vetter, I.R., and Wittinghofer, a (2001). The guanine nucleotide-binding switch in three dimensions. Science (New York, N.Y.) 294, 1299-1304.

Vimberg, V., Tats, A., Remm, M., and Tenson, T. (2007). Translation initiation region sequence preferences in Escherichia coli. BMC Molecular Biology 8, 100.

Voges, D., Watzele, M., Nemetz, C., Wizemann, S., and Buchberger, B. (2004). Analyzing and enhancing mRNA translational efficiency in an Escherichia coli in vitro expression system. Biochemical and Biophysical Research Communications 318, 601-614. 
Vohlander Rasmussen, L.C., Oliveira, C.L.P., Pedersen, J.S., Sperling-Petersen, H.U., and Mortensen, K.K. (2011). Structural transitions of translation initiation factor IF2 upon GDPNP and GDP binding in solution. Biochemistry 50, 9779-9787.

Voorhees, R.M., Schmeing, T.M., Kelley, A.C., and Ramakrishnan, V. (2010). The mechanism for activation of GTP hydrolysis on the ribosome. Science (New York, N.Y.) 330, 835-838.

Voorma, H.O., Benne, R., Naaktgeboren, N., and Van der Hofstad, G.A. (1979). Pleiotropic effects of initiation factor IF-1. Methods in Enzymology 60, 204-215.

Wakao, H., Romby, P., Westhof, E., Laalami, S., Grunberg-Manago, M., Ebel, J.P., Ehresmann, C., and Ehresmann, B. (1989). The solution structure of the Escherichia coli initiator tRNA and its interactions with initiation factor 2 and the ribosomal $30 \mathrm{~S}$ subunit. The Journal of Biological Chemistry 264, 20363-20371.

Wakao, H., Romby, P., Ebel, J.P., Grunberg-Manago, M., Ehresmann, C., and Ehresmann, B. (1991). Topography of the Escherichia coli ribosomal 30S subunit-initiation factor 2 complex. Biochimie 73, 991-1000.

Weber, A., Kögl, S. a, and Jung, K. (2006). Time-dependent proteome alterations under osmotic stress during aerobic and anaerobic growth in Escherichia coli. Journal of Bacteriology 188, 7165-7175.

Weiel, J., and Hershey, J.W. (1982). The binding of fluorescein-labeled protein synthesis initiation factor 2 to Escherichia coli $30 \mathrm{~S}$ ribosomal subunits determined by fluorescence polarization. The Journal of Biological Chemistry 257, 1215-1220.

Weiner, J., Herrmann, R., and Browning, G.F. (2000). Transcription in Mycoplasma pneumoniae. Nucleic Acids Research 28, 4488-4496.

Wienk, H., Tomaselli, S., Bernard, C., Spurio, R., Picone, D., Gualerzi, C.O., and Boelens, R. (2005). Solution structure of the C1-subdomain of Bacillus stearothermophilus translation initiation factor IF2. Protein Science : a Publication of the Protein Society 14, 2461-2468.

Wienk, H., Tishchenko, E., Belardinelli, R., Tomaselli, S., Dongre, R., Spurio, R., Folkers, G.E., Gualerzi, C.O., and Boelens, R. (2012). Structural dynamics of bacterial translation initiation factor IF2. The Journal of Biological Chemistry 287, 10922-10932.

Wilden, B., Savelsbergh, A., Rodnina, M. V, and Wintermeyer, W. (2006). Role and timing of GTP binding and hydrolysis during EF-G-dependent tRNA translocation on the 
ribosome. Proceedings of the National Academy of Sciences of the United States of America 103, 13670-13675.

Wimberly, B.T., Brodersen, D.E., Clemons, W.M., Morgan-Warren, R.J., Carter, A.P., Vonrhein, C., Hartsch, T., and Ramakrishnan, V. (2000). Structure of the $30 \mathrm{~S}$ ribosomal subunit. Nature 407, 327-339.

Winkler, W., Nahvi, A., and Breaker, R.R. (2002a). Thiamine derivatives bind messenger RNAs directly to regulate bacterial gene expression. Nature 419, 952-956.

Winkler, W.C., Cohen-Chalamish, S., and Breaker, R.R. (2002b). An mRNA structure that controls gene expression by binding FMN. Proceedings of the National Academy of Sciences of the United States of America 99, 15908-15913.

Wintermeyer, W., and Gualerzi, C. (1983). Effect of Escherichia coli initiation factors on the kinetics of N-Acphe-tRNAPhe binding to $30 \mathrm{~S}$ ribosomal subunits. A fluorescence stopped-flow study. Biochemistry 22, 690-694.

Wolfrum, A., Brock, S., Mac, T., and Grillenbeck, N. (2003). Expression in E. coli and purification of Thermus thermophilus translation initiation factors IF1 and IF3. Protein Expression and Purification 29, 15-23.

Wu, X.Q., and RajBhandary, U.L. (1997). Effect of the amino acid attached to Escherichia coli initiator tRNA on its affinity for the initiation factor IF2 and on the IF2 dependence of its binding to the ribosome. The Journal of Biological Chemistry 272, 1891-1895.

Yoo, J.-H., and RajBhandary, U.L. (2008). Requirements for translation re-initiation in Escherichia coli: roles of initiator tRNA and initiation factors IF2 and IF3. Molecular Microbiology 67, 1012-1026.

Yu, N.J., and Spremulli, L.L. (1997). Structural and mechanistic studies on chloroplast translational initiation factor 3 from Euglena gracilis. Biochemistry 36, 14827-14835.

Yusupov, M.M., Yusupova, G.Z., Baucom, a, Lieberman, K., Earnest, T.N., Cate, J.H., and Noller, H.F. (2001). Crystal structure of the ribosome at $5.5 \mathrm{~A}$ resolution. Science (New York, N.Y.) 292, 883-896.

Yusupova, G., Jenner, L., Rees, B., Moras, D., and Yusupov, M. (2006). Structural basis for messenger RNA movement on the ribosome. Nature 444, 391-394.

Yusupova, G.Z., Yusupov, M.M., Cate, J.H., and Noller, H.F. (2001). The path of messenger RNA through the ribosome. Cell 106, 233-241. 
Zheng, X., Hu, G.-Q., She, Z.-S., and Zhu, H. (2011). Leaderless genes in bacteria: clue to the evolution of translation initiation mechanisms in prokaryotes. BMC Genomics 12, 361.

Zhou, J., Lancaster, L., Trakhanov, S., and Noller, H.F. (2012). Crystal structure of release factor RF3 trapped in the GTP state on a rotated conformation of the ribosome. RNA (New York, N.Y.) 18, 230-240.

Zoldák, G., Sedlák, E., Wolfrum, A., Musatov, A., Fedunová, D., Szkaradkiewicz, K., and Sprinzl, M. (2008). Multidomain initiation factor 2 from Thermus thermophilus consists of the individual autonomous domains. Biochemistry 47, 4992-5005.

Zucker, F.H., and Hershey, J.W. (1986). Binding of Escherichia coli protein synthesis initiation factor IF1 to 30S ribosomal subunits measured by fluorescence polarization. Biochemistry 25, 3682-3690.

Zuker, M. (2003). Mfold web server for nucleic acid folding and hybridization prediction. Nucleic Acids Research 31, 3406-3415. 


\section{Aknowledgements}

I am very grateful to Marina Rodnina for providing me with great opportunities for professional development in science and motivating by being always the excellent example herself.

I am grateful to Wolfgang Wintermeyer for helpful discussions and his critical point of view.

I would also like to thank the members of my PhD Committee Ralf Ficner and Kai Tittmann for their time and discussions during our meetings.

I am grateful to Marat Yusupov and Gulnara Yusupova for a wonderful collaboration project, for creating motivating atmosphere in their lab and for being very welcoming during my stay in Strasbourg.

I would like to express my gratitude to Pohl Milon, from whom I learned a lot, for his help, advice and lots of discussions.

I am also very grateful to Andrei Konevega for being very friendly, patient answering all my questions and teaching me new techniques.

I am expressing my sincere gratitude to Natalia Demeshkina for sharing her scientific knowledge and lab experience with me during our discussions.

I am grateful to Lasse Jenner for introducing me to the exciting world of ribosome crystallography.

Many thanks to Dmitry Burakovskiy, Lena Palesskava, Ekaterina Samatova, Albena Draycheva, Michael Thommen and Tamara Seniushkina for being more than colleagues, but also very good and supportive friends. I am also grateful to all members of our lab for creating nice working atmosphere and being always friendly and helpful.

I specially thank Sandra Kappler, Christina Kothe, Olaf Geintzer, Tanja Wiles, and Theresia Uhlendorf for expert technical assistance and many preparations of ribosomes, tRNAs, and translation factors. I am also grateful to Dimitra Papastavrou for help in organizational affairs.

I am very grateful to Karine Santos for helping me with proofreading of my thesis. 
I am grateful to my friends and colleagues from Strasbourg, Sergey Melnikov, Nicolas Garreau de Loubresse, Irina Prokhorova and Iskander Khusainov for the great time that I had while working in the lab of Marat Yusupov.

My big thanks to my friends, whom I am very happy to have in my life and who shared a part of my PhD time with me: Alexander Kuklev, Vadim Alekseev, Anna Alekseeva, Arkadiy Alekseev, Olena Steshenko, Aliaksandr Dzementsei, Hanna Peradziryi, Andrei Shchebet, Fabio Neves. 


\section{Curriculum Vitae}

\section{Personal data}

Name Liudmila Filonava

Date of Birth 14.02.1982

Place of Birth Minsk, Belarus

\section{Scientific Education}

2009 - present PhD as a part of Göttingen Graduate School for Neuroscience, Biophysics and Molecular Biosciences; done in the Department of Physical Biochemistry, Max Planck Institute of Physical Biochemistry, Göttingen, Germany

2007 - 2009 MSc as a part of International Max Planck Research School for Molecular Biology and University of Göttingen; done in the Department of Physical Biochemistry, Max Planck Institute of Physical Biochemistry, Göttingen, Germany

2006 - 2007 Laboratory Assistant, Laboratory of Plant Functional Genetics, Academy of Science of the Republic of Belarus

2001 - 2006 Diploma work done in the Department of molecular biology, Belarusian State University, Minsk, Belarus

\section{School Education}

1997 - 2001 Undergraduate studies done at Linguistic College \#24, Minsk, Belarus. Qualified as a Teacher of English language. 1988 - 1997 School \# 24, Minsk, Belarus. 